\title{
Linking senses: the genetics of Drosophila larval chordotonal organs
}

\author{
Dissertation \\ for the award of the degree \\ "Doctor rerum naturalium" \\ of the Georg-August-Universität Göttingen
}

within the doctoral program IMPRS Neuroscience

of the Georg-August University School of Science (GAUSS)

Submitted by

Diego Alejandro Giraldo Sánchez

From Bogota, Colombia

Göttingen 2018 


\section{Thesis Committee}

Prof. Dr. Martin Göpfert

Department of Cellular Neurobiology, University of Göttingen, Göttingen

Prof. Dr. André Fiala

Department of Molecular Neurobiology of Behaviour, University of Göttingen, Göttingen

Dr. Manuela Schmidt

Somatosensory Signalling and Systems Biology Group, Max-Planck-Institute for

Experimental Medicine, Göttingen

\section{Members of the examination board}

Referee: Prof. Dr. Michael Hörner

Department of Cellular Neurobiology, University of Göttingen, and European

Neuroscience Institute (ENI), Göttingen

2nd Referee: Prof. Dr. Ralf Heinrich

Department of Cellular Neurobiology, University of Göttingen, Göttingen

3rd Referee: Dr. Roland Dosch

Department of Developmental Biochemistry, University Medical Center Göttingen, Göttingen

Date of oral examination: $13^{\text {th }}$ of June, 2018 
"Ok...Well, sometimes science is more art than science. A lot of people don't get that."

- Rick Sanchez

To Inés Forero de Sánchez and Hernando Sánchez 



\section{Table of contents}

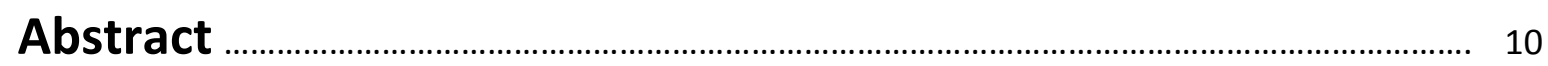

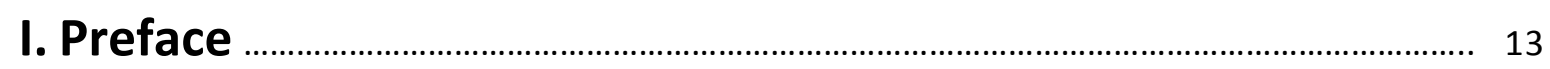

I.I. Transient receptor potential channels (TRP channels) .............................................. 13

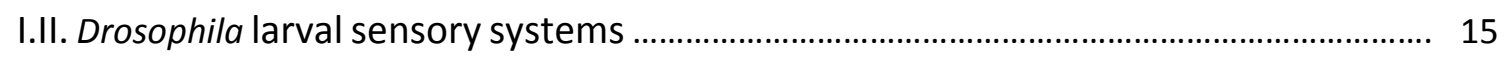

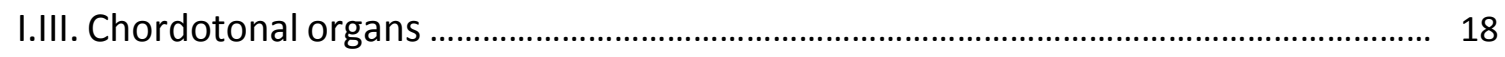

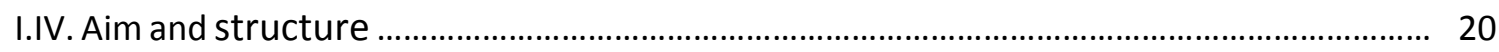

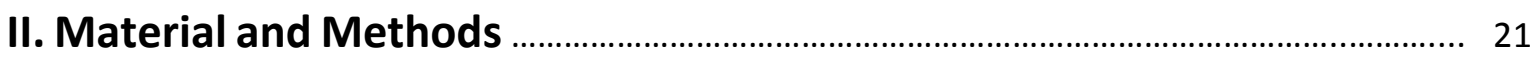

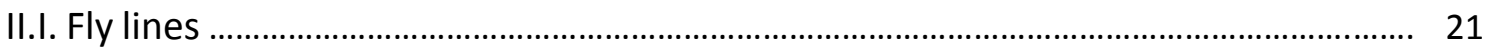

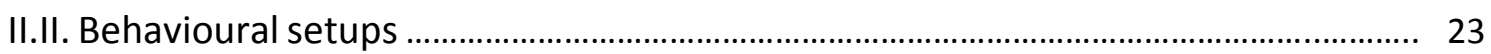

II.II.I. Frustrated total internal reflection setup (FTIR) ................................................... 23

II.II.II. Benzer gravitaxis setup .................................................................................... 23

II.II.III. Soldering iron-based temperature gradient setup .............................................. 24

II.II.IV. Peltier element-based temperature gradient setup .............................................. 25

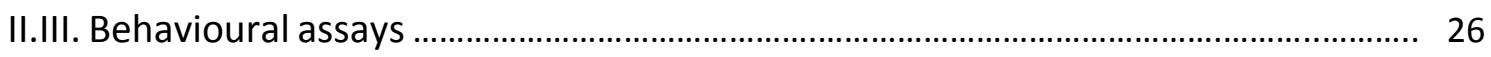

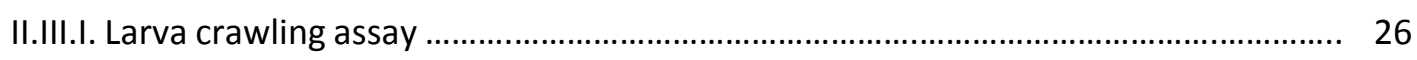

II.III.II. Larva peristalsis assays ................................................................................... 27

II.III.II.I. Mhc-GFP peristalsis assay ............................................................... 27

II.III.II.II. FTIR peristalsis assay ................................................................. 29

II.III.III. Adult locomotion assay ................................................................................ 29

II.III.IV. Temperature preferences of $3^{\text {rd }}$ instar larvae ................................................... 29

II.III.IV.I. Larval temperature preferences in a soldering iron-based setup ............ 29

II.III.IV.II. Larval temperature preferences in a Peltier element-based setup ........ 30

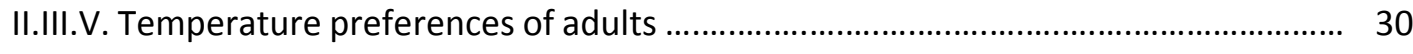

II.III.VI. Darth Vader assay ......................................................................................... 32

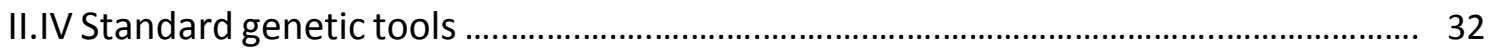


II.V. Creating a cold trapping correction model - IGLOO

II.V.I. Temperature dependence of locomotion

II.V.I.I. Larval temperature dependence of locomotion 33

II.V.I.II. Adult temperature dependence of locomotion 34

II.V.II. Temperature preferences of $3^{\text {rd }}$ instar larvae and adults 34

II.V.III. A null model for temperature-dependent locomotion 34

II.V.IV Temperature preference correction 35

II.VI. Genetic dissection of chordotonal larval proprioception 37

II.VI.I. Behavioural characterization of proprioception

II.VI.I.I. Larval crawling analysis 37

II.VI.I.II. Larval peristalsis analysis 38

II.VI.I.II.I. Mhc-GFP peristalsis analysis . 38

II.VI.I.II.II. FTIR peristalsis analysis 39

II.VI.II. Calcium imaging of larval chordotonal organs 39

II.VI.II.I. Fillet preparation 39

II.VI.II.II. Mechanical stimulation and imaging of Ich5 neurons 40

II.VI.II.III. Calcium imaging analysis 40

II.VII. Genetic dissection of larval chordotonal thermosensation 42

II.VII.I. Behavioural characterization temperature preferences

II.VIII. Immunohistochemistry of larval chordotonal organs 42

II.IX. Transmission electron microscopy of larval chordotonal organs ... 43

II.X. Neuronal responses using CaMPARI 44

II.X.I. CaMPARI function, set up and imaging 44

II.X.II. Chordotonal organ responses to temperature stimuli 45

II.XI. Statistical Analysis 45 


\section{Chapter 1: IGLOO - IGLOO is a Gradient LOcomotion mOdel: A model for temperature-dependent locomotion to correct cold trapping bias

1.1. Introduction

1.1.1. The anatomy and molecular mechanisms of thermosensation in Drosophila

1.1.1.1. Larval thermosensors .......................................................................... 48

1.1.1.2. Adult thermosensors …............................................................................ 49

1.1.2. Temperature preferences of Drosophila …............................................................. 50

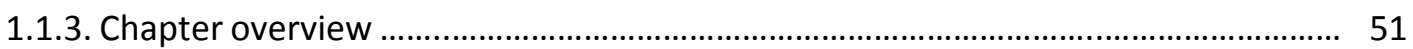

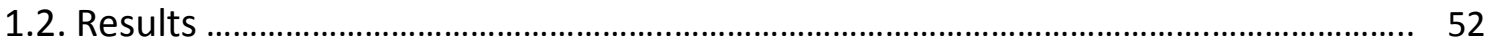

1.2.1. Velocity, bout duration, and activity depend on the local temperature ................. 52

1.2.2. Drosophila body thermodynamics ....................................................................... 54

1.2.3. Temperature dependence of locomotion fit ........................................................... 54

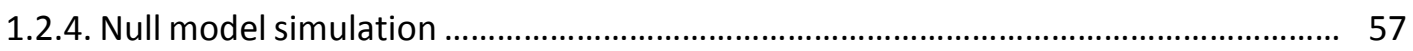

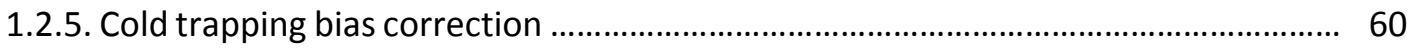

1.2.5.1. Temperature preference correction of larvae ........................................... 60

1.2.5.2. Temperature preference correction of adults .......................................... 62

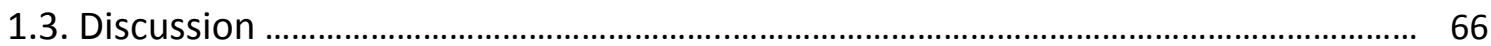

1.3.1 Ambient and rearing temperature influence locomotion ......................................... 66

1.3.2. Cold-trapping bias is corrected with IGLOO _............................................................. 69

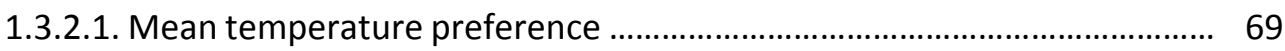

1.3.2.2. Range of tolerable temperatures .............................................................. 70

1.3.2.3. Start of cold and hot avoidance ……......................................................... 71

1.3.3. Temperature preferences of hot defective mutant larvae ....................................... 72

1.3.4. Temperature preferences of hot defective mutant adults ....................................... 73

1.3.5. Temperature preferences of cold defective mutant adults ..................................... 75

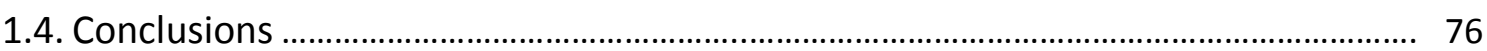




\section{Chapter 2: Proprioceptive functions of opsins in Drosophila larval chordotonal organs ............................................................................................................ 77}

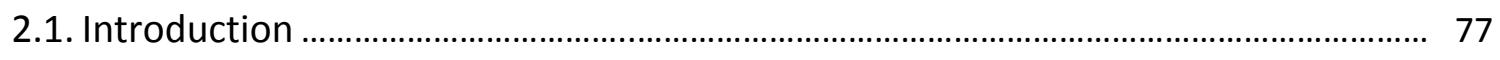

2.1.1. Drosophila opsins and visual transduction ........................................................... 77

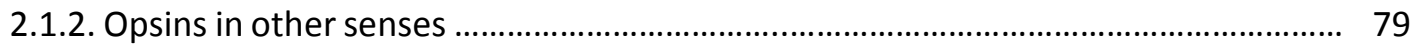

2.1.3. Larval chordotonal organs and mechanosensory channels .................................... 80

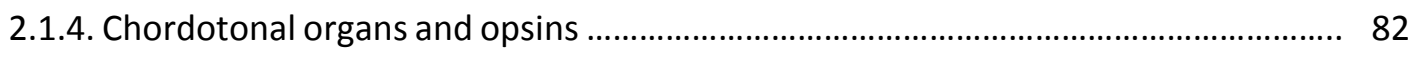

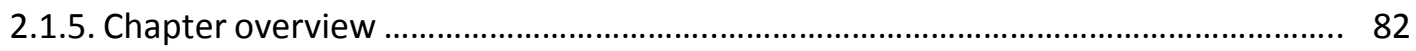

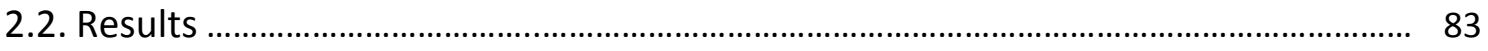

2.2.1. Rhodopsins are required for locomotion ……........................................................ 83

2.2.2. Chordotonal organs need Rhodopsin 1 for proper function ................................... 86

2.2.3. Rhodopsin function in chos is not related to thermosensation ................................ 90

2.2.4. Locomotion is retinal dependent ........................................................................... 93

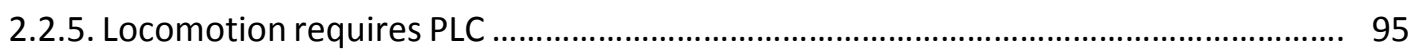

2.2.6. Cilium structure is affected by lack of Rhodopsin .................................................... 97

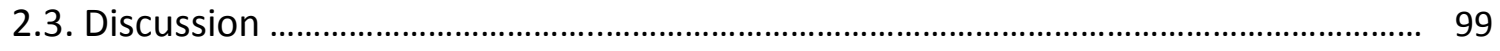

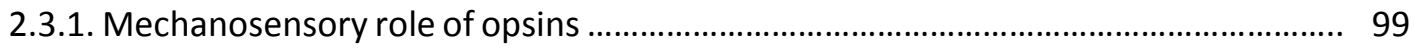

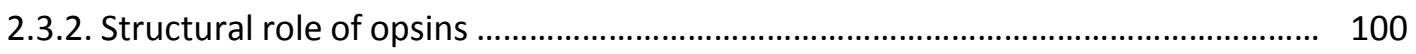

2.3.3. Retinal requirement for locomotion .................................................................. 101

2.3.4. PLC requirement for locomotion ......................................................................... 102

2.3.5. Ancient roles of opsins ...................................................................................... 102

2.4. Conclusions

Chapter 3: Larval chordotonal organs in thermosensation .............................. 105

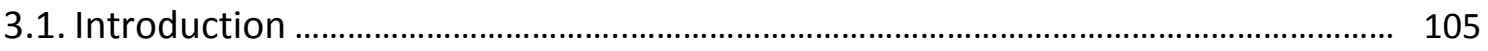

3.1.1. Chordotonal organs and thermosensation ........................................................ 105

3.1.2. Candidate molecules for thermosensation in larval chos ...................................... 107

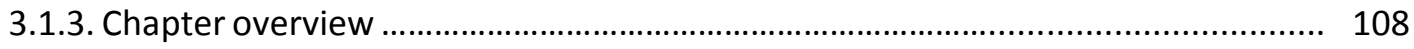


3.2.1. Thermosensory channel expression in chos

3.2.2. Brivido1 is not required in chos for thermosensation

3.2.3. Chordotonal organ responses to temperature

3.2.4. Brivido1 is not required in chos for locomotion

3.3. Discussion

3.3.1. Lch5 and thermosensation

3.3.2. Brivido1 expression in larval body wall neurons

3.4. Conclusions

4. General conclusions

5. References

6. Appendix

6.1. Solutions

6.2. Supplemental figures.

7. List of abbreviations

8. List of figures

9. List of tables

10. Acknowledgements 146

11. Declaration 148

12. Curriculum vitae 149 


\section{Abstract}

Sensory systems have evolved in all organisms to detect changes in the environment or their internal state. Even though they are essential for fitness and survival, their phylogenisis is still unclear. The protosensory cell hypothesis suggests that all sensory systems evolved from an ancestral cell that diversified throughout evolution and gave rise to all the senses present today. In recent years, evidence supporting the common ancestry of senses has accumulated. Sensory molecules that were initially believed to play a role only in one sensory modality have been found to be involved in various senses. For instance, thermosensors are involved in photo- and chemosensation, mechanosensors in thermosensation, or photoreceptors in mechanosensation. In addition, it has been found that cells that are mediating different senses have the same developmental origin, suggesting they diversified from the same ancestral cell.

Recently it was found that Rhodopsin (Rh), the photopigment involved in vision, was required for thermosensation and audition in the fly Drosophila melanogaster. In the course of this study, expression of two Rhs (Rh1 and Rh6) was detected in the larval pentameric chordotonal organ (Ich5), a group mechanosensory neurons of the larval body wall. This organ provides sensory feedback during locomotion and mediates touch and vibration sensation. Additionally, chordotonal organs (chos) have been implicated in the sensation of cool temperatures. The purpose of this study is to analyse the role of Rh1 and Rh6 in larval chos. Additionally, the role of Ich5 neurons in thermosensation was studied. Behavioural, physiological, and anatomical experiments were carried out to achieve these goals.

To study thermosensation, temperature preferences of larvae were tested in a temperature gradient. These preferences were found to be biased towards cold temperatures, because larvae cannot move away from them (i.e., cold trapped). To correct this cold trapping bias observed in temperature gradients, a null model of temperature-dependent locomotion IGLOO (Igloo is a Gradient LOcomotion mOdel) was generated. The effect of ambient temperature on locomotion of Drosophila larvae and adults was measured and used to simulate an animal with no preference walking in a gradient. The resulting distribution was successfully used to correct the cold trapping bias observed in temperature gradient behaviour.

Rh1 and Rh6 were found to be required for proper larval locomotion. This role was confirmed to be specific to chos by calcium imaging experiments and a tissue-specific knockdown. The structure of sensory cilia of Ich5 neurons was affected by the absence of Rh1, indicating that 
Rhs play a structural role in larval mechanosensory neurons. This is the first report of a rhabdomeric opsin playing a structural role in a ciliary cell. Since rhabdomeres in fly photoreceptors also require Rhs to maintain their structure, it now seems that Rhs played an ancestral structural role that predates their photosensory role in photoreceptor rhabdomeres.

Brivido1 - an ion channel involved in thermosensation - was found to be expressed in a subset of larval Ich5 neurons. Nonetheless, these neurons were not activated by cool or warm temperatures and a role of Brivido1 in cho proprioception was also excluded. Interestingly, Brivido1 was found to be coexpressed with NOMPC in Ich5, and two additional body wall neurons. This expression data suggests that Brivido1 may be interacting with NOMPC in these neurons, and modulating mechanosensation.

The structural ancestral role of Rhodopsin, and the presence of Brivido1 channels in a wide variety of sensory neurons, which mediate different senses in Drosophila, corroborates the protosensory cell theory. The data obtained suggests that different sensory systems share molecules that were most likely present in the ancestral cell they originated from. 


\section{Preface}

Living organisms rely on sensory information for their survival. Animals have evolved a wide variety of sensory systems that not only allows them to extract signals from their surroundings to find food, detect potential preys, or find conspecifics for reproduction, but also information about their internal states (e.g., temperature, limb position). However, how these sensory systems evolved is still not clear. It has been proposed that the nervous systems of bilaterians animals have a common origin (Ghysen, 2013).

A prominent hypothesis concerning the phylogenesis of sensory systems is that many of these systems derived from a "protosensory" cell (Niwa et al., 2004). Evidence for this can be found in Drosophila, where photoreceptors and chordotonal organs (mechanoreceptors comprising the ear and stretch receptors) were found to require the same proneural gene for development (atonal), suggesting that these two sensory systems have the same origin (Jarman et al., 1995; Niwa et al., 2004). Furthermore, genes of the Pax family have been found to be involved in the development of photoreceptors, mechanoreceptors and chemosensors of different species (Collinson et al., 2003; Fritzsch et al., 2005; Hartman et al., 2003).

In recent years more evidence of shared sensory molecules between senses has been found in Drosophila and has given additional support to the theory of the common origin of sensory systems. For example, visual opsins have been shown to be involved in thermosensation and audition (Senthilan et al., 2012; Shen et al., 2011; Sokabe et al., 2016), thermosensitive ion channels in photosensation and gustation (Kim et al., 2010; Xiang et al., 2010), and gustatory receptors in thermosensation and photosensation (Ni et al., 2013; Xiang et al., 2010). Proteins involved in the transduction cascade of one sense seem to be very often involved in other roles in different senses, therefore an in depth analysis of such "transducers" was the first step in this study.

\section{I.I. Transient receptor potential channels (TRP channels)}

The most prominent group of transducers is the superfamily of transient receptor potential (TRP) cation channels. These channels give additional evidence of the common ancestry of different senses. They are expressed in a wide variety of sensory receptors and allow for the transduction of different kinds of stimuli (Figure 1). Despite having different ion selectivity, modes of activation, and sensory and physiological functions, TRP channels have six transmembrane domains, high sequence similarities and are permeable to cations (Montell, 2005). The $\mathrm{N}$ and $\mathrm{C}$ terminus are 


\section{Preface}

located intracellularly, and a pore loop is present between the fifth and sixth transmembrane segments (Venkatachalam and Montell, 2007).

The three mechanisms of TRP channel activation are: receptor activation (e.g., G proteincoupled receptors and receptor tyrosine kinases), direct activation of the channel, and ligand activation (Ramsey et al., 2006). TRP channels are activated by different signals (e.g., mechanical, chemical or a secondary messenger cascade). However, one single TRP channel can be activated by more than one stimulus; for example, the mammalian TRPM8 channel is usually activated by cold temperature, but can also be activated by menthol (Dhaka et al., 2006; McKemy et al., 2002).

TRP channels can be divided into seven subfamilies: TRPA (ankyrin), TRPC (canonical), TRPM (melastatin), TRPML (mucolipin), TRPN (nompC), TRPP (polycystin), and TRPV (vanilloid) (Montell, 2005). All seven subfamilies are present in Drosophila (Figure 1), whereas mice and humans lack channels belonging to the TRPN subfamily (Fowler and Montell, 2013; Venkatachalam and Montell, 2007). An additional subfamily of TRP channels - the TRPY subfamily - was characterised in the yeast (Denis and Cyert, 2002; Palmer et al., 2001; Venkatachalam and Montell, 2007). The presence of a TRP channel in the yeast suggests that TRP channels were present before the evolution of metazoans, and provides further evidence of the common origin of sensory systems in animals.
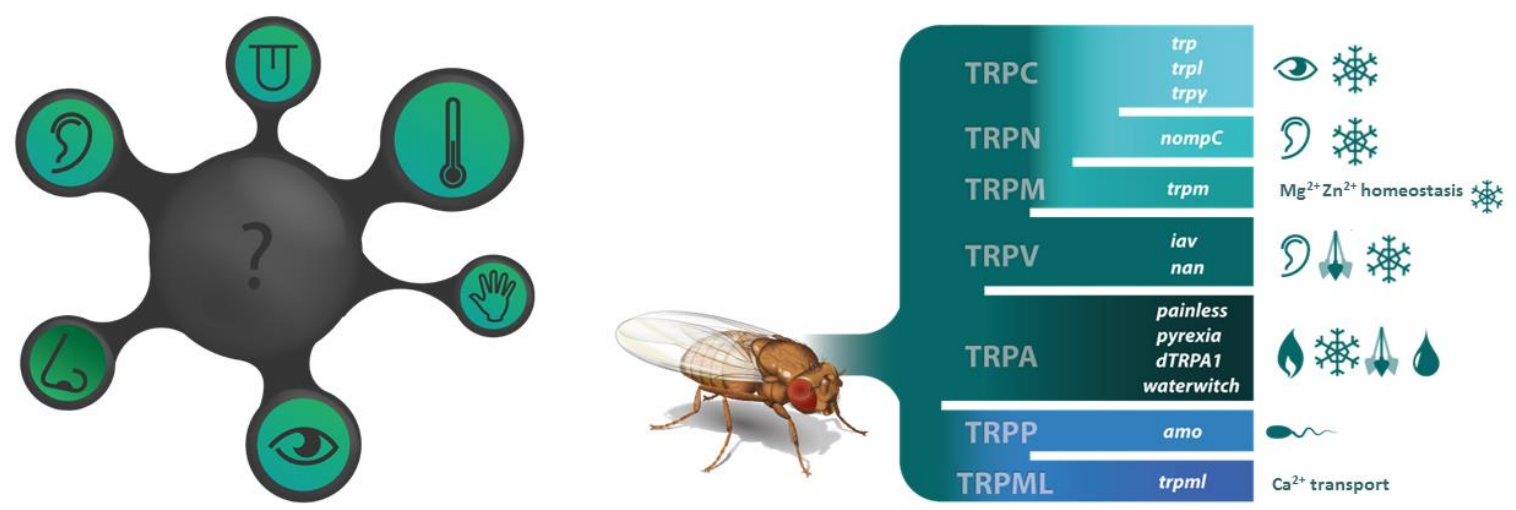

Figure 1. The protosensory cell theory and TRP channels. A) The protosensory cell theory suggests that all sensory systems evolved from the same ancestral cell with an unknown sensory function. This cell diversified and gave rise to the wide variety of sensory systems present in animals. Modified from Dr. Bart R. H. Geurten and Dr. Christian Spalthoff. B) Superfamily of TRP channels in Drosophila. All seven subfamilies are present in flies. The images on the right indicate what senses the channels are involved in. Modified from Dr. Christian Spalthoff, Montell, 2005, and Fowler and Montell, 2013.

In Drosophila, TRP channels have been found to be involved in a wide variety of senses (Figure 1, for a review see (Fowler and Montell, 2013)). Trp, Trpl, and Trpy are the three members of the TRPC subfamily and are involved in photosensation, proprioception and cold sensation (Akitake et al., 2015; Birnbaumer, 2009; Rosenzweig et al., 2008). The TRPN subfamily has one 
single representative, NOMPC, essential in senses involving mechanosensation like locomotion, touch, vibration detection, and audition (Cheng et al., 2010; Effertz et al., 2011; Yan et al., 2013; Zhang et al., 2013). The NOMPC channel has also been implicated in the sensation of noxious cold temperatures (Turner et al., 2016). The two representatives of TRPV subfamily are Nanchung and Inactive, which are also involved in mechanosensory senses (Gong et al., 2004; Kim et al., 2003; Sun et al., 2009; Zhang et al., 2013) and cold sensation (Kwon et al., 2010). Four channels of the TRPA subfamily have been characterised; Painless, dTRPA1, Pyrexia (dTRPA2) and Waterwitch (dTRPA3). These channels have been implicated in thermosensation (Rosenzweig et al., 2005), gustation (Kim et al., 2010), thermal nociception (Lee et al., 2005; Xu et al., 2006), mechanical nociception (Tracey et al., 2003), hygrosensation (Liu et al., 2007), and gravity sensation (Sun et al., 2009). The TRPM channel is involved in zinc and magnesium homeostasis (Georgiev et al., 2010; Hofmann et al., 2010), and cold nociception in larvae (Turner et al., 2016). In contrast to the aforementioned subfamilies, the TRPP and TRPML subfamilies have not been found to be involved in sensory processing. TRPML channels are important for calcium transport in the lysosomes (Wong et al., 2012), and the TRPP channel, Amo, has been implicated in sperm motility (Gao et al., 2003; Köttgen et al., 2011). Three channels, Brivido1-3, have been suggested to be TRPP channels and be involved in cold sensation in the adults (Gallio et al., 2011). However, it is still debated if they are part of the TRP channel superfamily since they have ten transmembrane domains instead of six (Fowler and Montell, 2013). More detail about Drosophila mechanosensory and thermosensory TRP channels will be given in Chapters 1-3.

\section{I.II. Drosophila larval sensory systems}

Drosophila larvae have been used to study the molecular mechanisms of different sensory systems. Since larvae are easy to handle and have a relatively simple nervous system, behavioural, physiological, and anatomical experiments can be easily carried out in large numbers of larvae that allow understanding the role of different molecules and groups of neurons in signal transduction. In addition to a dorsal brain and a ventral nerve chord, larvae have specialised sensory organs in the head and the body wall that allows them to extract information about the environment and their internal state (Fowler and Montell, 2013).

The larval head houses chemosensory receptors that are affiliated with a dorsal, a terminal, and a ventral organ (Python and Stocker, 2002; Singh and Singh, 1984). In addition to chemosensation, the terminal organ has been implicated in cold temperature sensation (Liu et al., 2003; Rosenzweig et al., 2008). The anterior part of the larva also houses the Bolwig's organ, a 
Preface

photosensory organ that mediates larval photosensation and circadian light entrainment (Green et al., 1993; Mazzoni et al., 2005).

The larval body wall has a group of sensory neurons that are specialised for different sensory modalities. These neurons are arranged in dorsal, lateral and ventral clusters in each hemisegment (Figure 2) (Brewster and Bodmer, 1995; Orgogozo and Grueber, 2005). There are two main classes of sensory neurons in the body wall: type I and type II (Kernan, 2007). Type I neurons have one dendrite with a cilium in the most distal tip and are usually surrounded by support cells (Kernan, 2007). The two kinds of type I neurons are external sensory organs (es) and chordotonal organs (chos) (Figure 2). Es comprise bristles, campaniform, and basiconical sensilla and sense mechanical and chemical stimuli (Brewster and Bodmer, 1995; Kernan et al., 1994). Chos are internal sensors and mediate proprioception, touch and sense vibrations (Caldwell et al., 2003; Fosque et al., 2015; Zhang et al., 2013). A more detailed description of chos will be given in the following section.

Multidendritic neurons (md) are type II neurons, which have multiple dendritic projections. These neurons can be classified in three major groups: dendritic arborisation neurons (da), bipolar dendrite neurons (bd), and tracheal dendrite neurons (td) that arborise around trachea (Figure 2) (Bodmer and Jan, 1987). Da neurons are divided into four morphological groups given their branching patterns: class I-IV neurons. Type II neurons have been implicated in a broad variety of senses including proprioception (Ainsley et al., 2003; Hughes and Thomas, 2007), cold temperature sensation (Liu et al., 2003; Turner et al., 2016), warm temperature sensation (Liu et al., 2003; Shen et al., 2011), noxious heat and touch (Tracey et al., 2003), and photosensation (Xiang et al., 2010). In addition to body wall neurons, thermosensory cells have been characterised in the larval brain (Hamada et al., 2008). 


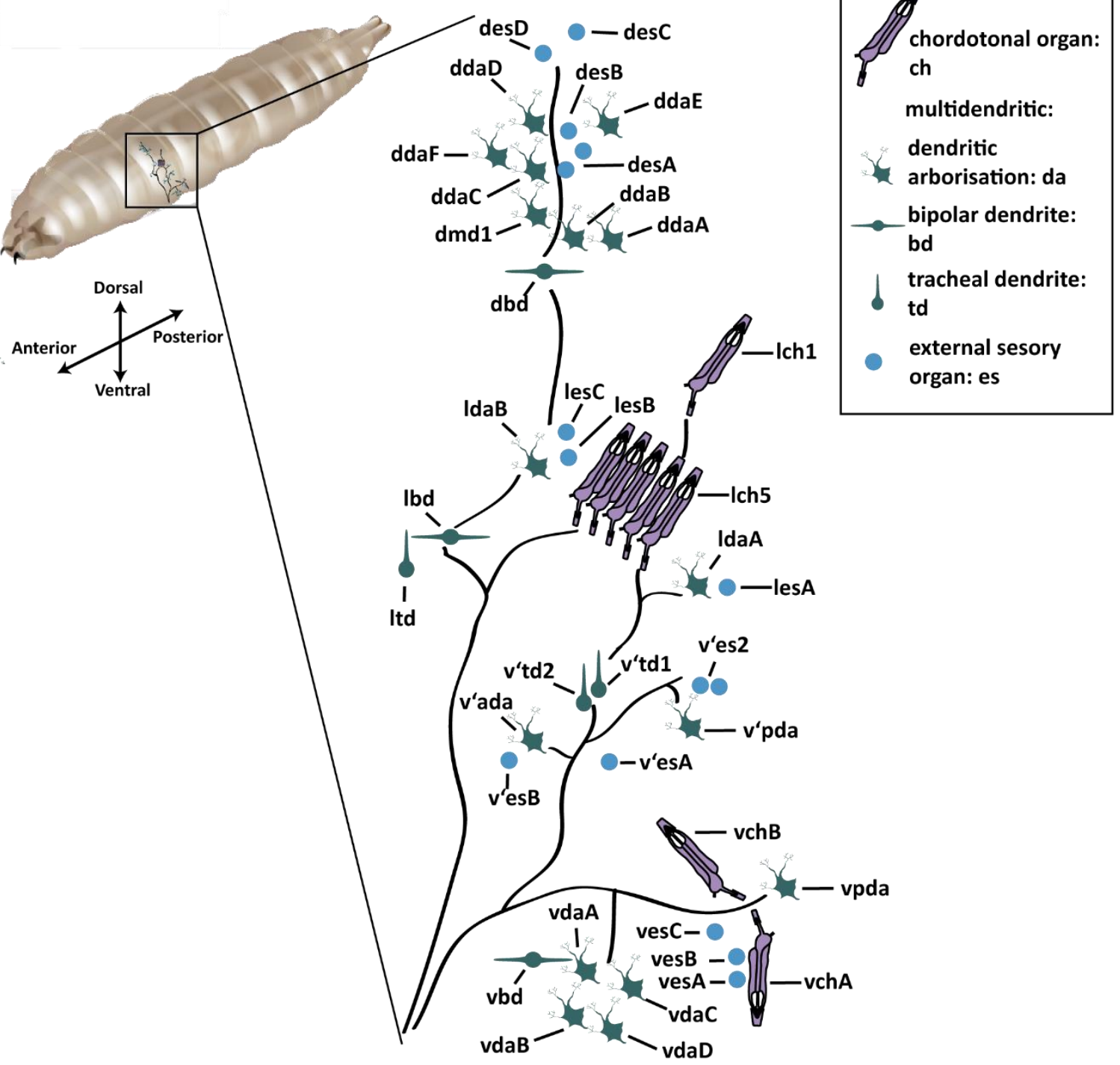

Figure 2. Sensory body wall neurons of a larval hemisegment. There are three kinds of sensory neurons in the larval body wall: chordotonal (ch, in purple), multidendritic ( $\mathrm{md}$, in green) and external sensory organs (es, in blue). The neurons are organised in dorsal (d), lateral (I) and ventral ( $v^{\prime}$ and $v$ ) clusters. Md neurons are divided into three types: dendritic arborisation (da), bipolar dendrite (bd) and tracheal dendrite (td). Modified from Brewster and Bodmer, 1995; Ghysen et al. 1986; Orgogozo and Grueber, 2005. The larva image was modified from an image by Dr. C. Spalthoff. 
Preface

\section{I.III. Chordotonal organs}

Chordotonal organs are type I sensory organs that comprise one to three monodendritic ciliated neurons surrounded by accessory cells (Figure 3C) (Eberl, 1999; Ghysen et al., 1986). The tip of chos is attached to the cuticle via cap cells, which transmit cuticular deflections to the sensory dendrites of the neurons, leading to the transduction of mechanical stimuli (Kavlie and Albert, 2013; Prahlad et al., 2017). A scolopale cell surrounds the dendrite and forms an endolymphatic space around the ciliary tip. The space contains endolymph which is high in potassium and resembles the endolymph space of the scala media of the mammalian ear (Field and Matheson, 1998; Göpfert and Henning, 2016; Kavlie and Albert, 2013). A third accessory cell, the ligament cell, anchors the organ proximally to the cuticle (Prahlad et al., 2017; Yack, 2004).

In the adult Drosophila, chos are serially arranged along the body, with the most prominent being found in the second antennal segment, where it forms the main auditory organ (the Johnston's organ) (Eberl, 1999; Jarman, 2002; Nadrowski et al., 2011). Another cho is the femoral segment of the leg, which provides proprioceptive feedback during locomotion and, in addition, mediates circadian entrainment to environmental temperatures (Chen et al., 2015; Shanbhag et al., 1992).

In larvae, chos are present in a group of neurons of the body wall (Figure 2 and Figure 3A, B). There are two single ventral chos (vchA and vchB), one single lateral (Ich1) and one lateral cluster of five, called the pentameric chordotonal organ (Ich5) (Figure 3B) (Brewster and Bodmer, 1995). Larval chos have been implicated in proprioception (Caldwell et al., 2003), touch (Caldwell et al., 2003; Scholz et al., 2015), and sensing vibrations (Fosque et al., 2015; Zhang et al., 2013). In addition to their mechanosensory roles, larval chos have been implicated in temperature sensation. However, the evidence provided for thermosensation is contradicting, with one study showing physiological responses of chos to warm temperatures (Liu et al., 2003), and the other reporting that larvae lacking the channel Inactive expressed in chos have reduced cold avoidance (Kwon et al., 2010). 
A
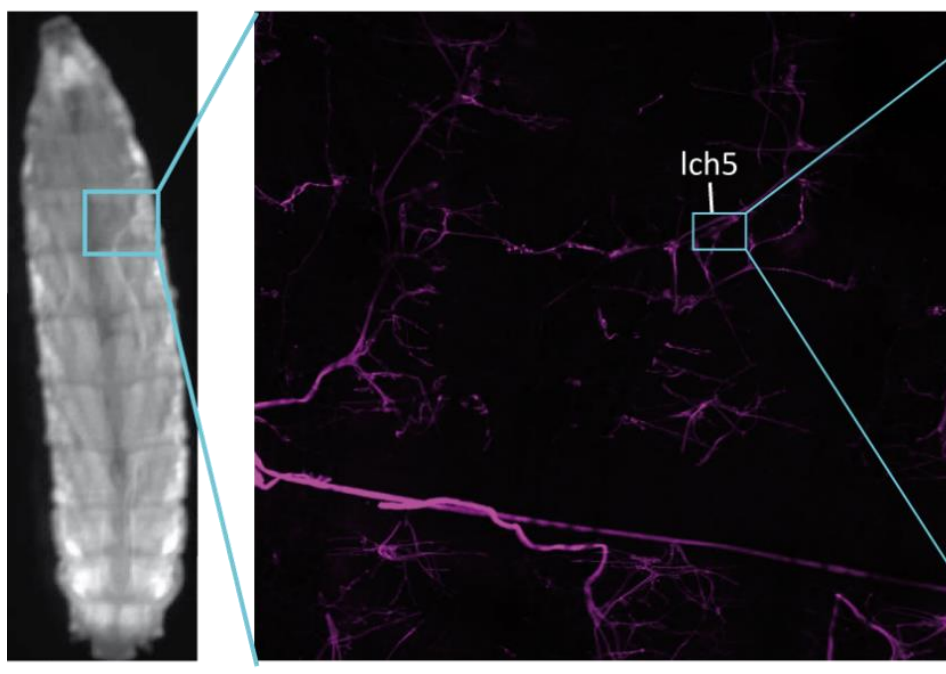

B

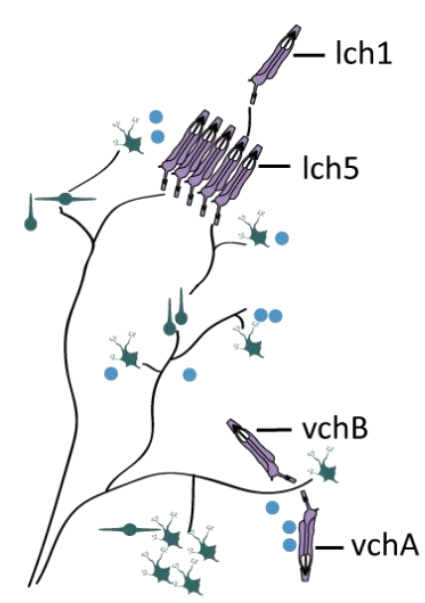

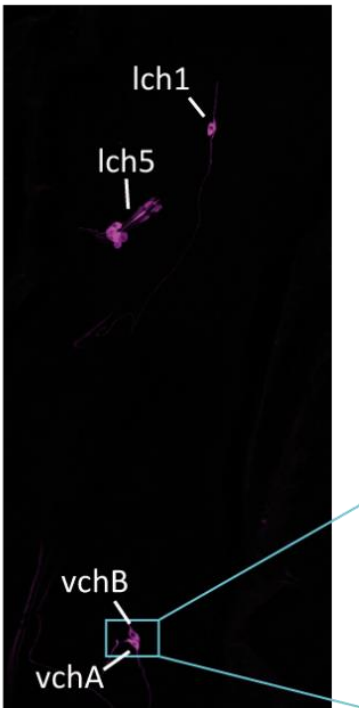

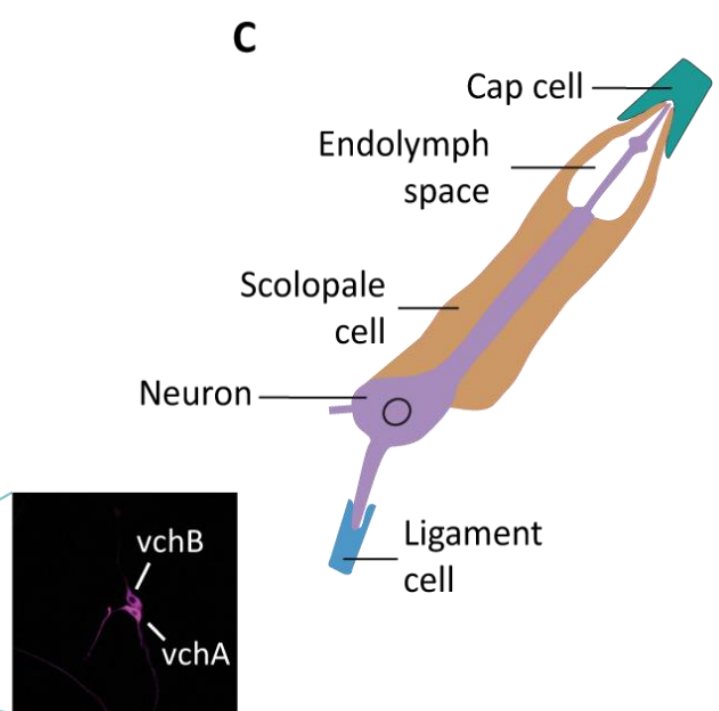

Figure 3. Larval pentameric chordotonal organs (Ich5). A) Image of the body wall neurons of a Drosophila larva stained with anti-HRP. In the middle panel, the body wall neurons of a hemisegment of a third instar larva are shown and the Ich5 is indicated. The panel on the right shows the five monodendritic neurons of the Ich5 expressing the fluorescent protein CaMPARI. B) Schematic of the lateral and ventral clusters of body wall neurons. The image shows the four kinds of body wall chos expressing CaMPARI using the cho specific Dhc93-Gal4 driver: one single lateral (Ich1), one lateral pentameric (Ich5), two single ventral (vchA and vchB). A zoom in of the ventral chos is shown. C) Schematic of a single chordotonal organ depicting the neuron and the accessory cells. 
Preface

\section{I.IV. Aim and structure}

In this study I set out to analyse the validity of the protosensory cell theory using Drosophila melanogaster larvae as an experimental model. For this purpose, I studied the role of genes involved in photosensation and thermosensation in larval proprioceptors (chordotonal organs). This thesis is divided into three chapters.

Chapter 1: To study thermosensation behaviourally I first had to solve the problem of cold trapping: the inability of flies and larvae to move away from a cold part region of a temperature gradient due to chill coma. In this chapter, I describe the generation of a null model that helps correct this cold trapping bias.

Chapter 2: Two visual opsins were found to be expressed in larval Ich5. I provide behavioural, physiological, and anatomical evidence for a role of these two opsins in proprioception in these organs.

Chapter 3: Larval chos have been implicated both in warm and cold temperature sensation. In this chapter, I studied the role of Ich5 in thermosensation and the role of Brivido (a cold sensing molecule found in adult cold receptors), in larval thermosensation and locomotion. 


\section{Material and Methods}

\section{II.I. Fly lines}

Table 1. Wild-type and mutant strains

\begin{tabular}{|c|c|c|}
\hline Strain & Genotype & Source \\
\hline Canton S & $+/+;+/+;+/+$ & Bloomington \\
\hline white & $w^{1118} ;+/+;+/+$ & $\begin{array}{l}\text { Provided by Frank } \\
\text { Sprenger }\end{array}$ \\
\hline ninaE mutant & $+/+;+/+; \operatorname{nin} a E^{17}, s r^{1}$ & Kyoto 109599 \\
\hline ninaE deletion & $w^{1118} ;+/+; D f(3 R) B S C 636 / T M 6 B$ & Bloomington 25726 \\
\hline ninaE rescue & $w ;+/+; \operatorname{nin} a E^{17}, s r^{1} / P\{$ nina $E\}$ & $\begin{array}{l}\text { Provided by Steve } \\
\text { Britt }\end{array}$ \\
\hline Rh6 mutant & $w ;+/+; R h \sigma^{1} / R h \sigma^{1}$ & 109600 (Kyoto) \\
\hline Rh6 rescue & $\begin{array}{l}y w^{67} ; C\left\{R h 6^{+}, y^{+} w^{+}\right\} / C y O ; R h 6^{1} / \\
T M 6 b\end{array}$ & $\begin{array}{l}\text { Provided by Claude } \\
\text { Desplan }\end{array}$ \\
\hline Rh6 deletion & $\begin{array}{l}w^{1118} ; D f(3 R) \text { Exel6174, } P\{X P- \\
\text { U\}Exel6174/TM6B, Tb1 }\end{array}$ & Bloomington 7653 \\
\hline nanchung mutant & $n a n^{36 a}$ & $\begin{array}{l}\text { Bloomington } 24902 \\
\text { (no longer in stock) }\end{array}$ \\
\hline inactive mutant & $i a v^{1}$ & $\begin{array}{l}\text { Bloomington } 6029 \text { (no } \\
\text { longer in stock) }\end{array}$ \\
\hline dtrpA1 mutant & $w ;+/+; \operatorname{dtrpA} 1^{i n s}$ & $\begin{array}{l}\text { Provided by Paul } \\
\text { Garrity }\end{array}$ \\
\hline santa maria mutant & $w ;$ santa-maria ${ }^{1} ;+/+$ & Bloomington 24520 \\
\hline norpA mutant & $w, \operatorname{norp} A^{36} ;+/+;+/+$ & Bloomington 9048 \\
\hline brv2 mutant & $\begin{array}{l}y^{1}, w ; M i\{y[+m D i n t 2]=M I C\} b r v 2^{M 104916} \\
/ T M 3, S^{1}, \operatorname{Ser}^{1}\end{array}$ & Bloomington 38067 \\
\hline$m h c-G F P$ & $y^{1} w^{*} ; P\{M h c-G F P . F 4-453\} 2 ;+/+$ & Bloomington 38462 \\
\hline
\end{tabular}


Table 2. Gal4 and UAS strains

\begin{tabular}{|c|c|c|}
\hline Strain & Description & Stock ID \\
\hline Dhc93AB-Gal4 & $\begin{array}{l}\text { Gal4 expression in chordotonal organ } \\
\text { neurons using the Dhc93AB } \\
\text { promoter. Chromosome } 2\end{array}$ & $\begin{array}{l}\text { Generated in Martin C. } \\
\text { Göpfert lab }\end{array}$ \\
\hline dnai2-Gal4 & $\begin{array}{l}\text { Gal4 expression in chordotonal organ } \\
\text { neurons using the dnai2 promoter. } \\
\text { Chromosome } 2\end{array}$ & $\begin{array}{l}\text { Generated in Martin C. } \\
\text { Göpfert lab }\end{array}$ \\
\hline $\operatorname{trpA-Gal4}$ & $\begin{array}{l}\text { Gal4 expression in dTRPA1 } \\
\text { expressing cells using the dtrpA1 } \\
\text { promoter. Chromosome } 3\end{array}$ & $\begin{array}{l}\text { Provided by Paul } \\
\text { Garrity }\end{array}$ \\
\hline CC-Gal4 (NP4486-Gal4) & $\begin{array}{l}\text { Gal4 expression using the brv1 } \\
\text { NP4486 allele as a promoter. It is } \\
\text { expressed in the cold sensing cells of } \\
\text { the adult arista. Chromosome } 3\end{array}$ & $\begin{array}{l}\text { Provided by Paul } \\
\text { Garrity }\end{array}$ \\
\hline HC-Gal4 (Gr28b.d-Gal4) & $\begin{array}{l}\text { Gal4 expression under the control of } \\
\text { the thermosensitive channel } \\
\text { promoter Gr28b.d. It is expressed in } \\
\text { the } 3 \text { hot cells of the arista. } \\
\text { Chromosome } 2\end{array}$ & $\begin{array}{l}\text { Provided by Paul } \\
\text { Garrity }\end{array}$ \\
\hline Ir25a-Gal5 & $\begin{array}{l}\text { Gal4 expression under the control of } \\
\text { the thermosensitive channel IR } 25 a \text {. }\end{array}$ & \begin{tabular}{|l}
$\begin{array}{l}\text { Provided by Richard } \\
\text { Benton }\end{array}$ \\
\end{tabular} \\
\hline norpA-Gal4 & $\begin{array}{l}\text { Gal4 expression under the control of } \\
\text { norpA }\end{array}$ & Bloomington 45016 \\
\hline UAS-ninaERNAi & $\begin{array}{l}\text { Expresses interference RNA for ninaE } \\
\text { under UAS control. Chromosome } 3\end{array}$ & Bloomington 31647 \\
\hline UAS-hid,rpr & $\begin{array}{l}\text { Expresses the apoptotic factors Hid } \\
\text { and Reaper under UAS control. } \\
\text { Chromosome X }\end{array}$ & $\begin{array}{l}\text { Provided by John } \\
\text { Nambu }\end{array}$ \\
\hline UAS-brv1RNAi & $\begin{array}{l}\text { Expresses interference RNA for brv1 } \\
\text { under UAS control. Chromosome } 3\end{array}$ & Bloomington 31506 \\
\hline UAS-6XGFP & $\begin{array}{l}\text { Expresses GFP in high amounts under } \\
\text { UAS control. Chromosome } 2\end{array}$ & Bloomington 52261 \\
\hline UAS-CAMPARI & $\begin{array}{l}\text { Expresses CaMPARI under UAS } \\
\text { control. Chromosome } 2\end{array}$ & Bloomington 58761 \\
\hline
\end{tabular}

Table 3. LexA and LexAop strains

\begin{tabular}{|l|l|l|}
\hline Strain & Description & Stock ID \\
\hline R57C10-LexA- & Expresses GCaMP6f panneuronally & Rebalanced from a line \\
LexAop-GCaMP6f/CyO-weep & $\begin{array}{l}\text { under LexAop control using LexA } \\
\text { expression with the neuronal } \\
\text { synaptobrevin promoter R57C10. } \\
\text { Chromosome 2 }\end{array}$ & \\
\hline
\end{tabular}




\section{II.II. Behavioural setups}

\section{II.II.I. Frustrated total internal reflection setup (FTIR)}

To obtain high contrast videos of larvae crawling, a modified frustrated total internal reflection (FTIR) setup was used (Risse et al., 2013). The setup consisted of agar (1\% agarose) placed on top of a Plexiglas pane that was illuminated with infrared LED's (wavelength $850 \mathrm{~nm}$, Narrow beam Dragon dome LED, OSRAM Opto Semiconductors GmbH, Germany) from the side (Figure 4A, B). Total internal reflection happens when a wave of light hits a medium boundary at an angle exceeding a critical angle, whereby the refractive index of the medium before the boundary must be larger than that of the medium behind. Because the refractive index of the Plexiglas is higher than that of the air, light is reflected inside the Plexiglas and does not pass to the air (Risse et al., 2013). If a third medium is added that has a larger refractive index, the light is no longer reflected but passes through to the third medium in a process called frustrated total internal reflection. Since the agar placed on the Plexiglas has higher refractive index than that of the Plexiglas, the light passes to the agar (Risse et al., 2013). Finally, because a larva has a larger refractive index than the agar, light passes from the agar to the larva. Thus the larva appears to be the only object lighting up in a completely black background, maximising the contrast and minimising common tracing problems (Risse et al., 2013) (Figure 4B). A camera (Teledyne Dalsa Motion Traveller 300, IS Imaging Solutions $\mathrm{GmbH}$, Eningen, Germany) placed under the set up recorded the larva crawling on the agar.

\section{II.II.II. Benzer gravitaxis setup}

To test the temperature dependence of locomotion in adult flies a modified Benzer gravitaxis test was used (Benzer, 1967). Taking advantage of the negative gravitaxis of flies (Gargano et al., 2005), knocking flies to the bottom of a vertical arena and allowing them to walk up can be used to test the speed of locomotion of adults. A vertical arena with 15 separate tracks $(73.5 \mathrm{~mm} \times 4.5 \mathrm{~mm})$ was printed with a 3D-printer (Ultimaker 1, Ultimaker BV, Geldermalsen, The Netherlands) (Figure 4C). The arena was closed with a Plexiglas pane and placed in a 3D-printed frame. The arena was slid up and down in the frame, to knock the flies to the bottom of the tracks. White light was used for illumination, and a camera (Hercules Optical Glass, Guillemot Cooperation, La Gacilly, France) was used to record the flies walking up the tracks (Figure 4D). 


\section{II.II.III. Soldering iron-based temperature gradient setup}

To assess temperature preferences of Drosophila larvae and adults a temperature gradient was generated in an aluminium slab divided into five tracks of $50 \mathrm{~mm} \times 3 \mathrm{~mm} \times 3 \mathrm{~mm}$ (Figure 5A). The cold side of the gradient was generated by placing a brass cylinder containing ice water with salt that was kept at $-80^{\circ} \mathrm{C}$. The hot side was made by heating four soldering irons that were inserted inside the arena. The irons were set to heat up one side of the arena to a specific temperature. Once the desired temperature was reached the irons would turn on and off to keep temperature fluctuations close to the desired temperature.

A

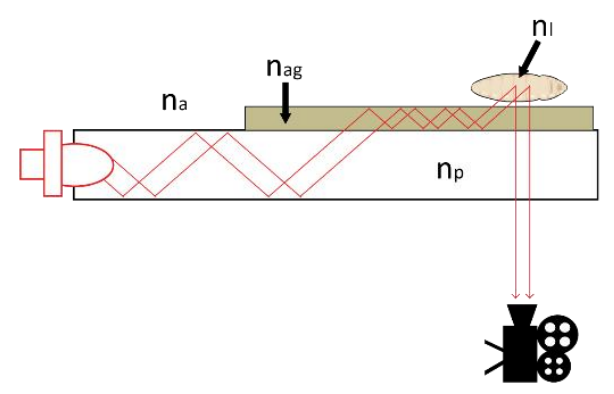

C

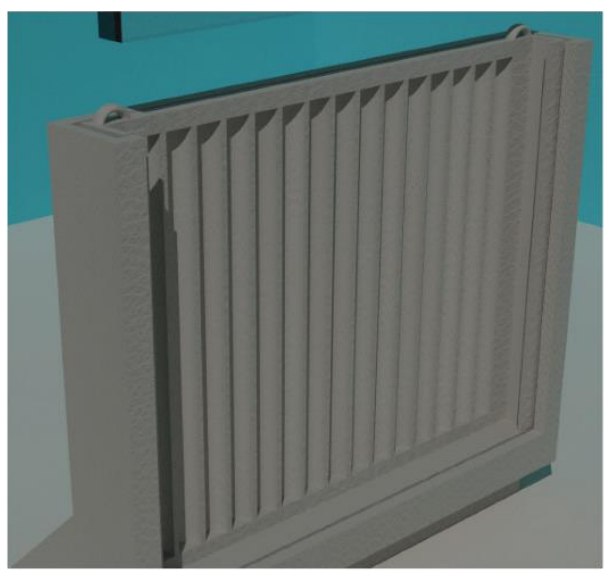

B

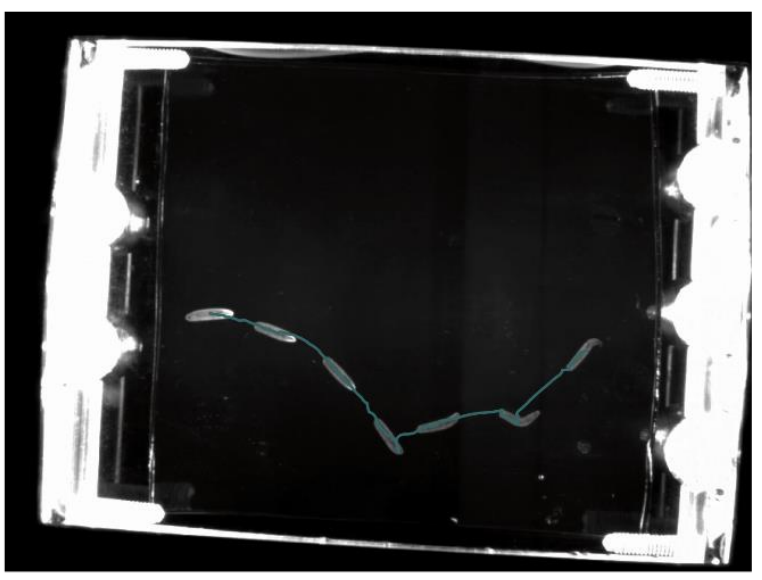

D

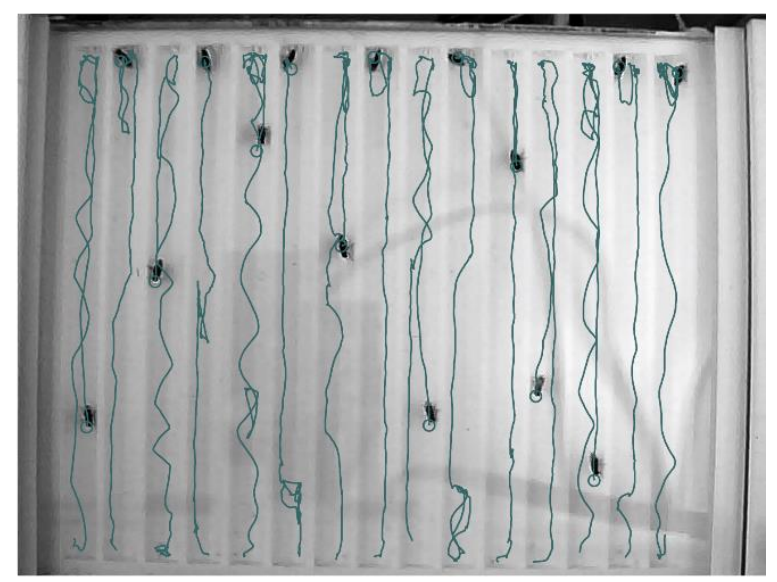

Figure 4. Larva and adult locomotion setups and tracing. A) FTIR setup for larval crawling. The refraction index (RI) of Plexiglas $\left(n_{p}\right)$ is higher than the air $\left(n_{a}\right)$ so the light is reflected in the glass. Since the RI of the agar placed on the glass $\left(n_{a g}\right)$ is higher than $n_{p}$, light passes to the agar. Since the RI of the larva $\left(n_{1}\right)$ is higher than $n_{a g}$, the light goes into the larva and is reflected back to the camera, maximizing contrast and allowing to have the lights inside the glass, and for the set up to go in an incubator with controlled temperature. Image modified from Risse et al. 2013 and Dr. Christian Spalthoff. B) Example trace of a larva crawling on the FTIR setup. C) Benzer setup for adult locomotion. A 3D printed arena divided into 15 tracks is placed in a frame that allows sliding the arena up and down to knock the flies down to the bottom of the tracks. Modified from Dr. Bart R. H. Geurten. D) Example trace of adult flies walking up the tracks. 
30 thermoresistors inside the aluminium slab (SEMI833ET, B+B Thermo-Technik GmbH, Donaueschingen, Germany) recorded the temperature of the arena at $10 \mathrm{~Hz}$. A voltage value was obtained via a Wheatstone bridge for each thermoresistor. The output of the sensors was recorded using a multiplexer that was controlled by an Arduino micro (D'Ausilio, 2012). The voltage value was then converted on the Arduino to the corresponding temperature value. The microcontroller sent the data using a serial communication protocol to a PC running Matlab (The MathWorks, Natick, MA, USA). The temperature was monitored live on a graphical user interface running on Matlab and was saved for further analysis. During the recordings, the arena was illuminated with infrared light (940nm \pm 25 ; Bausatz Infrarot-Scheinwerfer, Pollin Electronic GmbH, Pförring, Germany) that cannot be detected by the larvae.

\section{II.II.IV. Peltier element-based temperature gradient setup}

An additional setup that used Peltier elements (PEs) was used to assay temperature preferences. Four PEs (Thermoelectric module QC-63-1.0-3.9, Quick-Ohm, Germany) were aligned and placed under an aluminium plate of $80 \mathrm{~mm} \times 65 \mathrm{~mm}$ (Figure 5B). The PEs were controlled using an Arduino chip. A cooling fluid (Double Protect Ultra, Aqua Computer, Benniehausen, Germany) was pumped under the PEs (pump: Aquacomputer Aquastream XT, Aqua Computer, Benniehausen, Germany) to generate a heat sink and allow the PEs to generate cold temperatures. The temperatures of each PE were controlled with a program running on Arduino IDE (Arduino cc.). In addition, 30 thermoresistors (SEMI833ET, B+B Thermo-Technik GmbH, Donaueschingen, Germany) on the aluminium plate would record the temperatures and show them live on the program running on Arduino IDE, to monitor the temperatures at different points in the arena. An agar (1\% agarose) could be placed on the aluminium plate to provide a better surface for crawling and walking. The arena was divided into five tracks with a plastic separator that could be placed on top of the agar, which allowed to test either group or individual temperature preferences. Finally, a Plexiglas was placed on top of the tracks to prevent the animals from leaving the arena. 
A

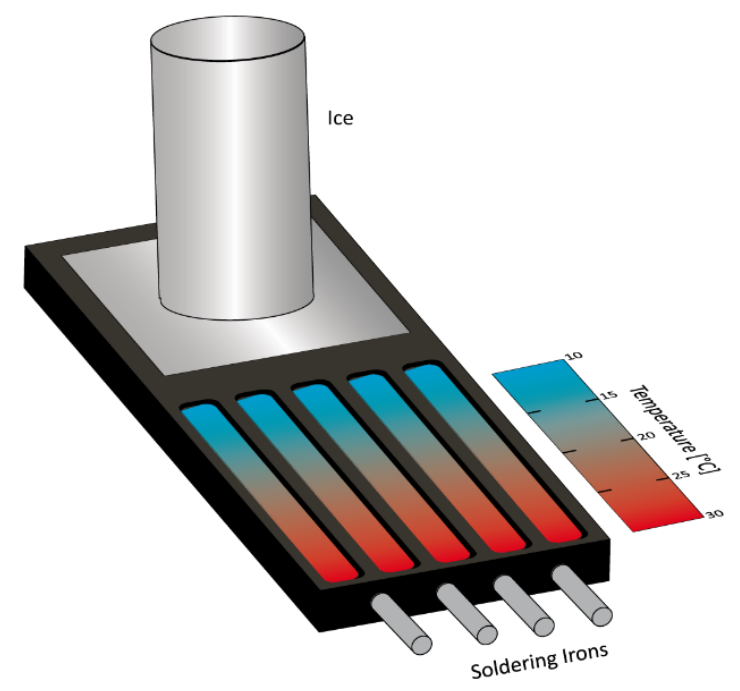

B

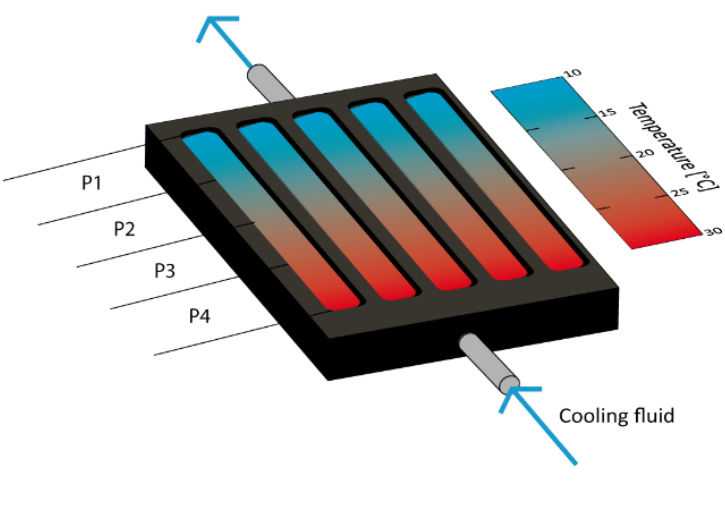

Figure 5. Temperature preference setups. A) Soldering iron-based setup. A temperature gradient is generated on an aluminium slab by heating one side with soldering irons and cooling the other side with a brass cylinder containing ice. The arena is divided into 5 tracks to test animals individually. (B) Peltier element-based setup. 4 PEs (P1-P4) are aligned to generate a temperature gradient. Each element is set to a specific temperature and agar is placed on top. A cooling fluid flows under the PEs generate a heat sink for the cooler part of the gradient. The agar is then divided into 5 tracks to test individual preferences. A Plexiglas (not shown) is placed on top of the tracks to avoid the animals from escaping in both setups. Note: not to scale.

\section{II.III. Behavioural assays}

\section{II.III.I. Larva crawling assay}

To describe larval locomotion the FTIR setup (see section II.II.I) was used. The setup was placed in an incubator (DigiTherm, Tritech Research Inc., Los Angeles, USA) that allowed to control the ambient temperature. Since the light emitted by the LED's cannot be detected by the larvae (Montell, 2012), the recordings were done in complete darkness for them.

Wandering $3^{\text {rd }}$ instar larvae were placed on the agar inside the incubator after being washed twice in water to remove any food residues. Once the larvae began crawling, they were recorded at 50fps using Troublepix (NorPix Inc., Montreal, Canada) for 3 minutes or until they reached the edge of the experimental arena. The traces of crawling larvae were reconstructed using ivTrace (Jens P. Lindemann, Bielefeld University) and analysed with Matlab R2012b (see Figure 4B for an example trace). 


\section{II.III.II. Larva peristalsis assays}

\section{II.III.II.I. Mhc-GFP peristalsis assay}

To study peristaltic contractions of larvae the Mhc-GFP method (Hughes and Thomas, 2007) was implemented. Larvae expressing GFP in muscles using a myosin heavy chain promoter (Mhc) were washed twice in water to remove any food residues and placed on a petri dish filled with agar ( $1 \%$ agarose). The petri dish was on an inverted fluorescence microscope (IX73, Olympus, Japan) illuminated with a mercury-vapour-lamp (U-HGLGPS, Olympus, Japan). Larvae were imaged while crawling for up to 10 seconds at $1.25 \mathrm{X}$ magnification and 100fps, with a GFP filter and a CCD camera (OptiMos, Qlmaging, Roper, USA) using Micro-Manager (Edelstein et al., 2014). The larva was kept in focus by manually adjusting the microscope stage. If the larva reached the edge of the arena or was out of focus the recording was stopped. As the muscles contract, more molecules of GFP come close together generating an increased fluorescent signal that allowed to see the propagation of the peristaltic contraction wave (Figure 6A, B). In addition, individual segments could be distinguished, allowing to quantify individual segment contractions. The recorded videos were analysed with Matlab2012b. 
A

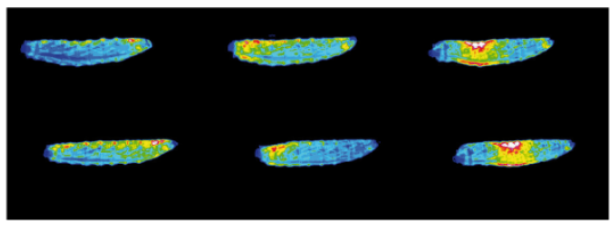

C

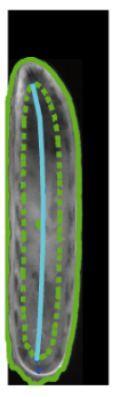

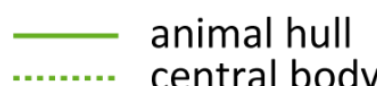

central body center of mass central line inner line

D

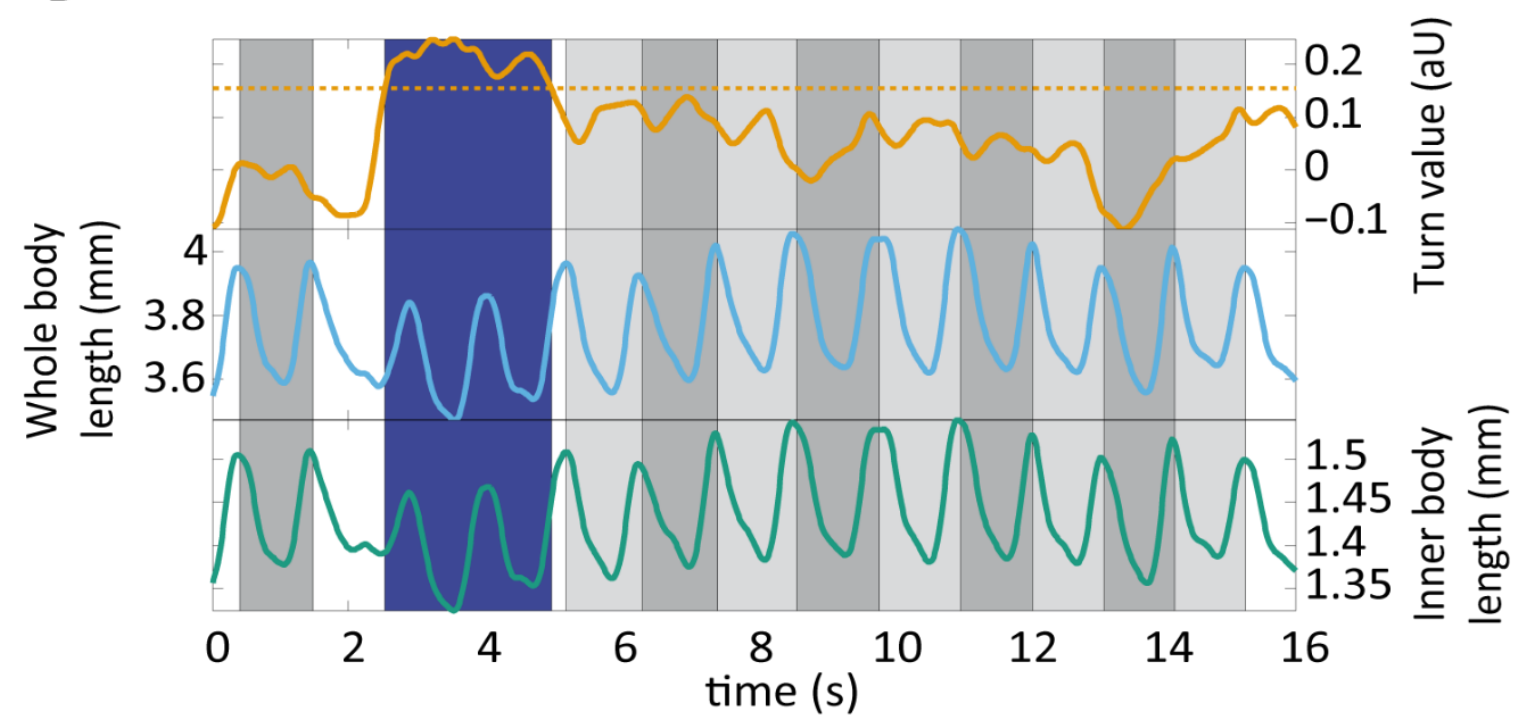

Figure 6. Larval peristalsis analysis assays. A) Wave propagation in a wild-type larva expressing GFP in muscles. Six frames of a 3.2 second recording are shown. Blue indicates low fluorescence intensity because the muscles are relaxed and red indicates high fluorescence intensity because the muscles are contracted. The changes in fluorescence are used as an indirect measure to calculate peristaltic contractions. B) Schematic explaining the images shown in A. During elongation, the muscles are relaxed and the GFP molecules are far resulting in low fluorescence intensity. During contraction, the muscles in the segments contracted become shorter, resulting in a reduced distance between the GFP molecules and higher fluorescence intensity measured. Larvae images modified from Dr. Christian Spalthoff. C) FTIR image of a larva crawling with high magnification (1.25X). The lines indicate all the features that can be automatically detected from each video frame. D) Calculations of peristaltic movements obtained from the features in C for a single larva. The upper graph shows the turning values calculated. The yellow dotted line indicates the threshold for turning detection. The frames with a turning value above the threshold are not used in the analysis (blue area). The whole body length (middle graph) and inner body length (lower graph) are used to calculate the amplitude of contraction. 


\section{II.III.II.II. FTIR peristalsis assay}

The FTIR setup described in section II.II.I was also used to study peristaltic contractions. The setup was placed on top of an inverted microscope (IX73, Olympus, Japan) and the larvae were imaged crawling for 45 seconds or until the larva either reached the edge of the arena or was out of frame at 1.25X magnification and 34fps with a CCD camera (OptiMos, QImaging, Rope, USA) using MicroManager (Edelstein et al., 2014). The larva was kept in focus by manually adjusting the microscope stage. The high contrast videos obtained with the FTIR setup allowed to see the entire body movements and were used to quantify the peristaltic contractions during crawling (Figure 6C, D). The recorded videos were analysed with Matlab2012b.

\section{II.III.III. Adult locomotion assay}

Adult flies were anesthetised on ice and one fly was placed in each of the 15 tracks of the Benzer gravitaxis assay (see section II.II.II) and was given 10 minutes to recover at room temperature. The arena was placed in the same incubator used for the larva crawling assay in section II.III.I. The flies were knocked down to the bottom of the tracks and were recorded at 30fps as they walked to the top. The recording was stopped as soon as the last fly reached the top and 5 repetitions were carried out. The traces of walking adult flies were obtained using ivTrace (Jens P. Lindemann, Bielefeld University) and analysed with Matlab R2012b (see Figure 4D for an example trace).

\section{II.III.IV. Temperature preferences of $3^{\text {rd }}$ instar larvae}

\section{II.III.IV.I. Larval temperature preferences in a soldering iron-based setup}

To assess the temperature preferences of late $3^{\text {rd }}$ instar larvae, the larvae were allowed to crawl under infrared illumination in a temperature gradient generated in the setup described in section II.II.III. The recordings were done using infrared illumination. The ice-containing brass cylinder was placed on the aluminium slab opposite to the soldering irons. After two minutes, the Matlab programme was started. Once the cold temperature reached $3^{\circ} \mathrm{C}$ the soldering irons were switched on and were set to fluctuate between $29.5^{\circ} \mathrm{C}$ to $30.5^{\circ} \mathrm{C}$.

Once the gradient was generated (cold part around $10^{\circ} \mathrm{C}$ ) $3^{\text {rd }}$ instar wandering larvae were washed twice in water to remove any food residues, and were placed into the hot end of the arena tracks (a total of 5 larvae per experiment). After 8 minutes the larvae were recorded crawling in the gradient for 5 minutes at $50 \mathrm{fps}$. 


\section{Material and Methods}

The traces obtained from the recording allowed obtaining the $X$ and $Y$ position of the larvae in the gradient (Figure 7A). The temperature at the 30 sensors was used to interpolate the temperature at the centre of mass of the larvae for all the recorded positions (spatial interpolation). Since the video recording was carried out at $50 \mathrm{fps}$, and the temperature data was obtained at $10 \mathrm{~Hz}$, also a temporal interpolation was undertaken. This resulted in a combination of the trajectory and the temperature data - i.e., a temperature trace - representing the larval ambient temperature over time (Figure 7B). The mean temperature of each larva was calculated as a measure of the individual temperature preference.

\section{II.III.IV.II. Larval temperature preferences in a Peltier element-based setup}

The Peltier element-based setup described in section II.II.IV allowed generating a stable gradient through time on an agar (1\% agarose) that enabled the larvae to crawl better in the gradient than in the soldering iron-based setup (section II.II.III). Once the gradient was generated, a 5-track division was placed on the agar so that larval temperature preferences could be tested individually. A $3^{\text {rd }}$ instar wandering larva was placed in each track and allowed to crawl around the gradient for 10 minutes in complete darkness. Once the 10 minutes had passed, the lights were turned on and a photo of the larvae in the arena was taken to obtain their final $X$ and $Y$ positions in the gradient (Figure 7E). The temperature of the thermoresistors in the arena at the end of the experiment was saved. To obtain the temperature at each pixel of the arena the temperatures of adjacent sensors was interpolated as in section II.III.IV.I. The position of the larvae was then combined with the temperature data to obtain the temperature each larva was at the end of the experiment (Figure 7F).

\section{II.III.V. Temperature preferences of adults}

To assess temperature preferences of adults, female virgin flies were allowed to walk in a temperature gradient generated in the soldering iron-based setup described in section II.II.III. Females were chosen because they are larger and easier to trace, and virgins were used to avoid any changes in preference due to oviposition site search (Dillon et al., 2009). Virgins were anesthetised with $\mathrm{CO}_{2}$ and given at least $24 \mathrm{~h}$ to recover. Only flies younger than 5 days were tested. The gradient was set as in section II.III.IV.I. In the meantime, the flies were anesthetised on ice. Once the gradient was generated, one fly was placed in the hot end of the arena tracks (a total of 5 flies per experiment). After 10 minutes the flies were recorded walking in the gradient for 5 minutes at 50fps. The traces were then combined with the temperature data to obtain the temperature of each fly through time as explained for larvae in section II.III.IV.I (Figure 7C, D) to 
calculate the mean temperature of each larva as a measure of the individual temperature preference.

A

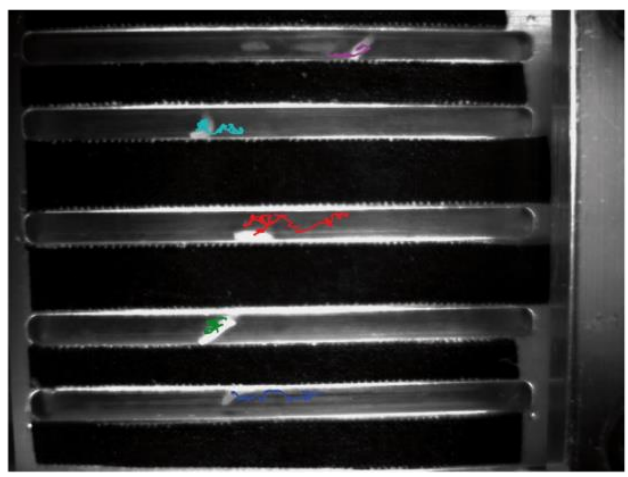

C

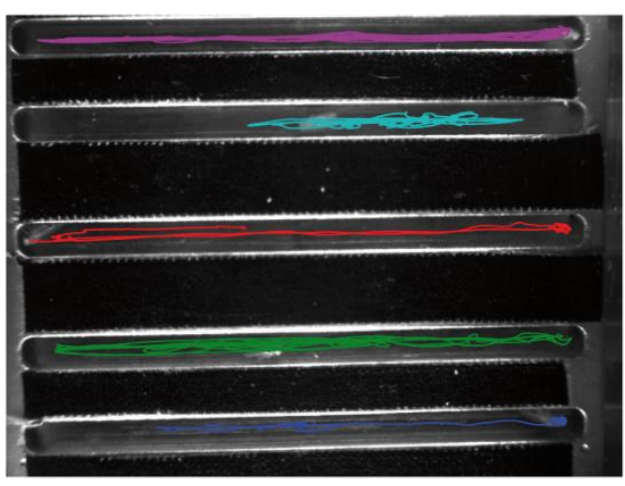

$\mathbf{E}$

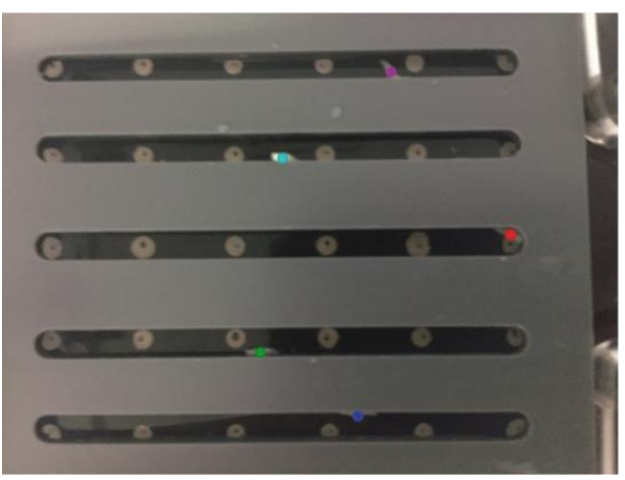

B

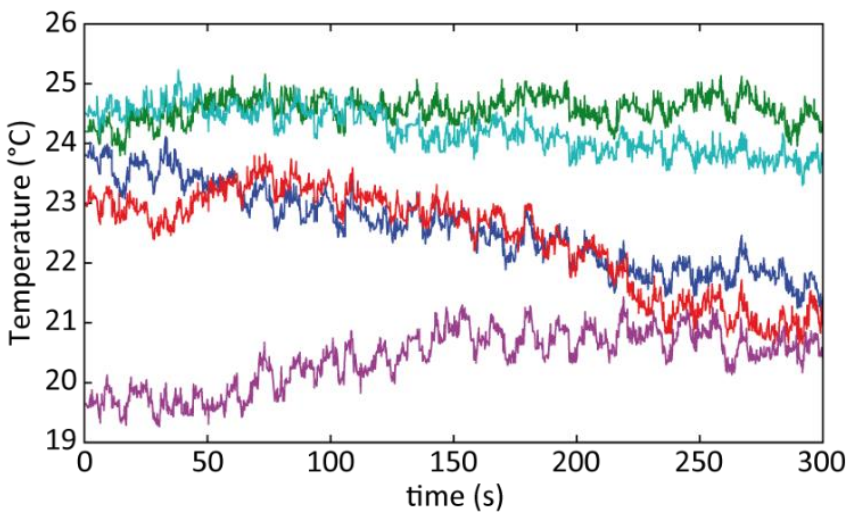

D

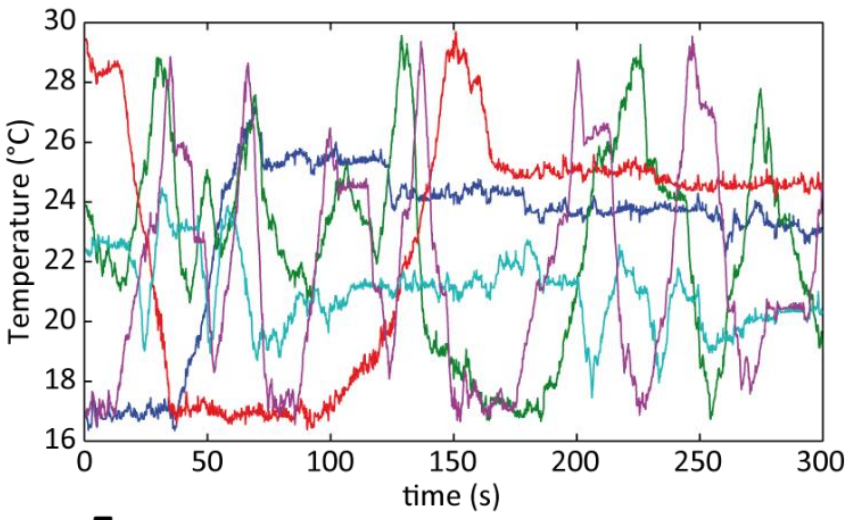

$\mathbf{F}$

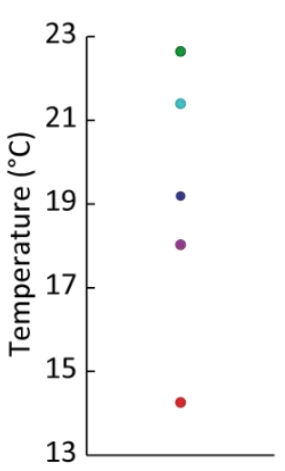

Figure 7. Example temperature traces. A) Example trace of larvae in a soldering iron-based setup. A single frame is shown with the full trace plotted for each larva. B) Temperature trace of the larvae shown in A. C) Example trace of adults in a soldering iron-based setup. A single frame is shown with the full trace plotted for each fly. D) Temperature trace of the flies shown in C. E) Image of the final position of larvae after 10 minutes in a Peltier element-based setup. F) Final temperatures of the larvae shown in $\mathrm{E}$. 


\section{II.III.VI. Darth Vader assay}

The Darth Vader assay was used to test for photoreception deficits in larvae (Dettman et al., 2001). The protocol was modified as in Caldwell et al. 2003. Half of a petri dish was painted black, and the other half was illuminated from underneath by placing it on an acrylic pane that had white light LED's under it (Figure 8). Aluminium foil was also placed under the dark side to ensure that no light would go through. The petri dish was filled with agar (1\% agarose). A single larva was placed on the agar in the boundary between the light and dark side and was allowed to choose between both sides for 90 seconds or until it reached the edge of the arena. A chi-square test was used to test if the choices of either side had the same probability.

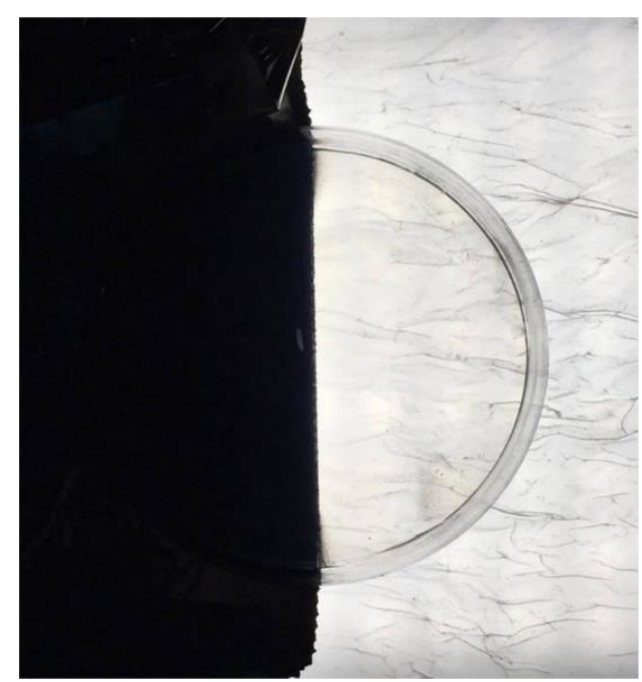

Figure 8. Darth Vader assay arena. A Petri dish with agar is placed on an acrylic pane with white light LED's. Half of the dish is painted black and covered with aluminium foil. A larva is placed in the middle and allowed to choose between dark and light.

\section{II.IV Standard genetic tools}

Drosophila offers a wide variety of genetic tools that allow the study of different genes in biological processes. Flies and larvae carrying mutations in specific genes were used to study the role of the proteins encoded by such genes in proprioception and thermosensation. The Gal4/UAS system (Brand and Perrimon, 1993; Duffy, 2002) and the LexA/LexAop system (Lai and Lee, 2006) were used to target gene expression in a tissue-specific manner. These tools were used in combination with interference RNA (RNAi) to silence gene expression in specific tissues (Fire et al., 1998; Perrimon et al., 2010), and they were also used to express the apoptotic factors Hid and Reaper for neuronal ablation (Grether et al., 1995; White et al., 1994). To measure neuronal responses to specific stimuli calcium imaging was used with the genetically encoded ultrasensitive calcium sensor GCaMP6f (Chen et al., 2013). 


\section{II.V. Creating a cold trapping correction model - IGLOO}

Temperature gradients can be used to assess temperature preferences of Drosophila larvae and adults. Small ectothermic animals such as Drosophila are very sensitive to changes in the environmental temperature and will have reduced locomotion speed at cold temperatures. This results in the animals spending more time at colder temperatures of the gradient and a cold biased preference, not because of an actual preference to cold temperatures, but a physiological inability to move away from colder temperatures (i.e., cold trapping). IGLOO (Igloo is a Gradient Locomotion Model) is a null model of a larva or adult without any temperature preference walking in a temperature gradient to correct the cold trapping bias.

\section{II.V.I. Temperature dependence of locomotion}

To assess how temperature affects locomotion of Drosophila, locomotion speed and bout duration of larvae and adults were obtained at different temperatures. A frustrated total internal reflection setup was used for larvae and a modified Benzer gravitaxis assay was used for adults.

\section{II.V.I.I. Larval temperature dependence of locomotion}

Wild-type Cantons larvae were reared at $25^{\circ} \mathrm{C}$ at $60 \%$ humidity in a $12 \mathrm{~h} / 12 \mathrm{~h}$ light-dark cycle. The FTIR setup (see section II.II.I) was placed in an incubator (DigiTherm, Tritech Research Inc., Los Angeles, USA) that allowed to control the ambient temperature. The larva crawling assay described in section II.III.I was used.

Larval locomotion was tested at temperatures ranging from $8^{\circ} \mathrm{C}$ to $40^{\circ} \mathrm{C}$. At very low temperatures where the larvae did not crawl, the recording was started 1 minute after the larva was placed in the arena. A total of 20 larvae were recorded for each temperature.

The traces were analysed to obtain the mean crawling speed, overall activity, and bout duration. The crawling speed of larvae was calculated for the entire duration of the recording, and the bout duration was defined as the duration of crawling before stopping for more than 1 second. Activity was determined as the time when the animal's forward speed was higher than $10 \%$ of its body length per second. 


\section{II.V.I.II. Adult temperature dependence of locomotion}

To test the temperature dependence of locomotion of adult flies the adult locomotion assay described in section II.III.III was used. Wild-type CantonS flies were reared at $18^{\circ} \mathrm{C}$ and $25^{\circ} \mathrm{C}$ at $60 \%$ humidity in a $12 \mathrm{~h} / 12 \mathrm{~h}$ light-dark cycle. Flies were tested at temperatures ranging from $6^{\circ} \mathrm{C}$ to $45^{\circ} \mathrm{C}$ and a total of 30 flies was tested per temperature for both groups $\left(18^{\circ} \mathrm{C}\right.$ reared and $25^{\circ} \mathrm{C}$ reared). In temperatures where flies were less capable of walking the recording was stopped once the flies clearly had settled in a specific position and did not walk further upwards.

The traces were analysed to obtain the mean speed of locomotion, overall activity, and bout duration. To obtain the mean speed of locomotion only upward movements were taken into account and calculated as the difference between all positive vectors, with sideways and downward movements being ignored. Bout duration was defined the time of upward movement without stopping for more than 1 second. Activity was calculated as the time when the animal's upward speed was higher than $10 \%$ of its body length per second.

\section{II.V.II. Temperature preferences of $3^{\text {rd }}$ instar larvae and adults}

Larvae were reared at $25^{\circ} \mathrm{C}$ and $60 \%$ humidity in a $12 \mathrm{~h} / 12 \mathrm{~h}$ light-dark cycle, while adults were reared at $18^{\circ} \mathrm{C}$ and $25^{\circ} \mathrm{C}$ in the same humidity and light-dark cycle. Temperature preferences of wild-type and mutants with temperature sensation deficiencies were tested using the soldering iron-based assay described in section II.III.IV.I for larvae and II.III.IV for adults. The gradient generated ranged from $14^{\circ} \mathrm{C}( \pm 1)$ to $30^{\circ} \mathrm{C}( \pm 0.5)$. The density probability at each temperature and the mean temperature preference was calculated for each strain.

\section{II.V.III. A null model for temperature-dependent locomotion}

A fly with no temperature preference walking around a temperature gradient was simulated using a Random Walk Monte Carlo-type (RWMC) model. The model worked on the assumption that the fly would walk around the gradient in a random fashion, and the speed and bout duration of locomotion at each step were influenced by the local temperature. These parameters were empirically obtained from the temperature dependence of locomotion experiments (section II.V.I.I for larvae and II.V.I.II for adults). Additional assumptions were: i) the gradient has one dimension, it is linear and stable through time, ii) the borders of the gradient are reflective, and iii) the probability of moving to hot or cold is the same at every point of the gradient. 
The temperature dependence of locomotion data was used to fit the probability of having a certain velocity $v$ and bout duration $d$ given the animal's temperature influenced by the local temperature $t$ and rearing temperature $T$. Once the parameters of the fit were obtained, the simulation was run. First, to obtain the body temperature of the animal a thermodynamic conduction model was used. Both larvae and adults were simulated as a cylinder of water with a length of $2 \mathrm{~mm}$ and a radius of $0.5 \mathrm{~mm}$ (final surface $7.85 \mathrm{~mm}^{2}$ ). The change in temperature was modelled using a heat flow formula (Equation 1), where $\lambda$ is the heat conductance of water (0.6 $\mathrm{W} / \mathrm{m}^{2 *} \mathrm{~K}$, (Ramires et al., 1995)), $\mathrm{A}$ is the surface, $\mathrm{D}$ is the wall to wall thickness, and $\mathrm{T}$ the ambient temperature, and $\mathrm{t}$ is the body temperature. The heat flow (units Joule/s) was multiplied by the bout duration ( $t$, unit seconds) resulting in a value of unit Joule.

Equation 1. Heat flow formula: $Q=\left(\lambda * \mathbf{A} * \frac{T 1-T 2}{D}\right) * t$

Because 1 Joule heats $1 \mathrm{~g}$ of water by 0.2449 , and Drosophila weights about $0.25 \mathrm{mg}$, the energy $Q$ obtained can be used to calculate the temperature change of the animal given the local temperature.

At each step of the simulation, the simulated animal would first choose the direction of movement (i.e., towards hot or cold). Then, given its calculated body temperature it would move with a velocity $\mathrm{x}$ and for a duration $\mathrm{y}$, resulting in a given distance of movement and a new local temperature reached (Figure 9). The probabilities of moving with a velocity $x$ for a duration $y$ were also derived from the fit and were dependent on the local temperature. A total of 1000 animals were simulated walking around a gradient for 2 hours for each group (larvae, $18^{\circ} \mathrm{C}$ reared adults, and $25^{\circ} \mathrm{C}$ reared adults), resulting in a histogram of the distribution around the gradient of the animal without a temperature preference (i.e., the null distribution).

\section{II.V.IV Temperature preference correction}

The calculated temperature preference histograms from the experiments in section II.V.II were corrected for the cold trapping bias using the null distribution obtained from the simulation (section II.V.III). For this purpose, the simulated distribution was subtracted from the calculated distribution of each animal tested, which resulted in temperatures having positive and negative distribution values. These new distribution values were defined as the preference index (PI). Temperatures with a negative PI were considered as avoided, whereas temperatures with a positive PI were considered as tolerated of preferred. Temperatures at which the $95 \%$ confidence interval of the PI overlapped with 0 were regarded as neither preferred nor avoided and not included in the temperature 


\section{Material and Methods}

preference analysis. The mean preferred temperature was then calculated only from those temperatures with a positive PI. In addition, the start of cold and hot avoidance was calculated as the temperatures where there was a change from a positive PI to a negative one. Finally, the difference between the mean preferred temperatures before and after correction was calculated to measure the effect the correction had in the initial calculation.

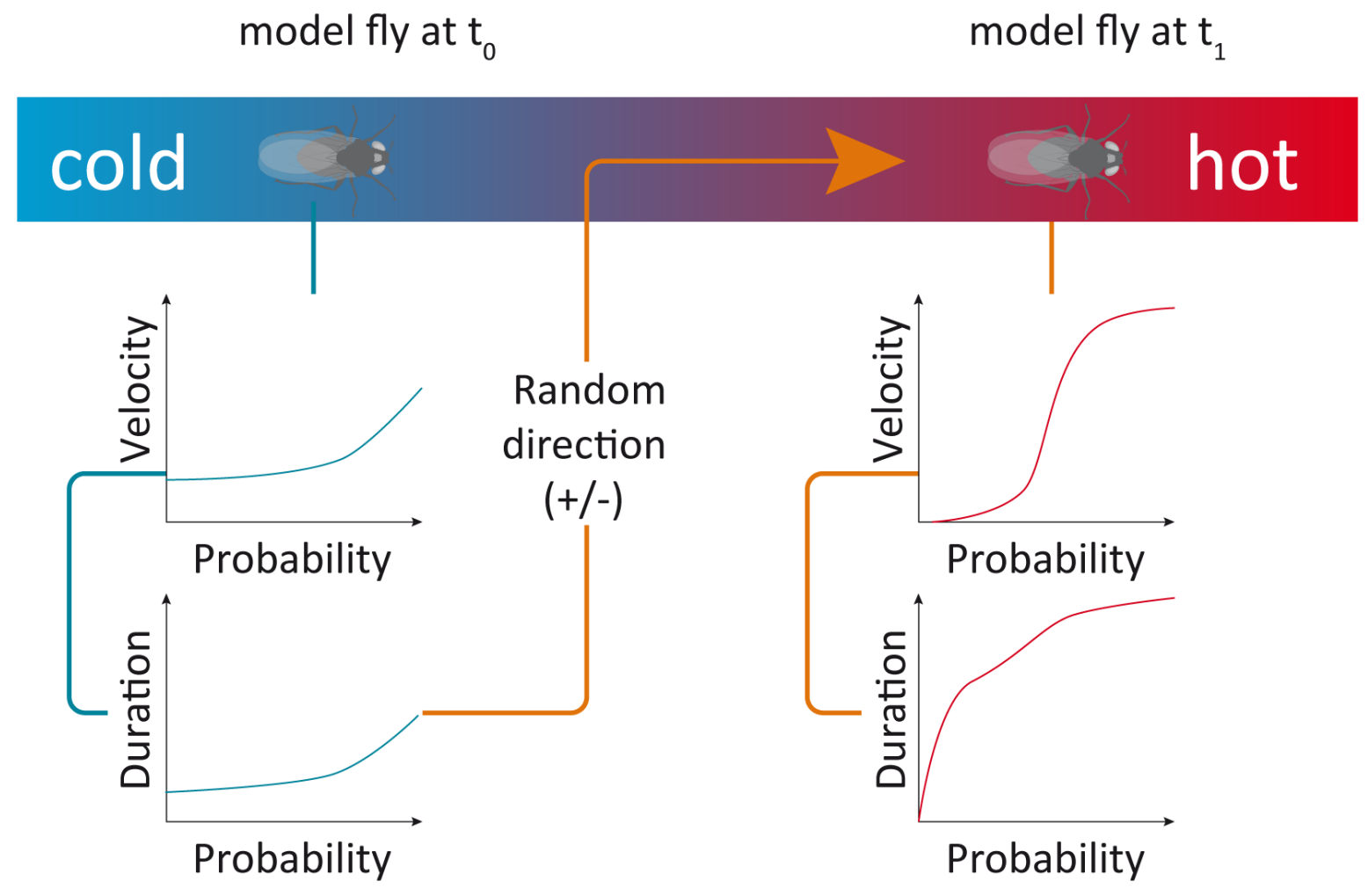

Figure 9. A null model for temperature-dependent locomotion. A step in the simulation of a fly with no temperature preference walking around a temperature gradient is depicted. The fly at $t_{0}$ moves to a second temperature $\left(t_{1}\right)$. Given the temperature dependence of locomotion, the speed and duration of movement are influenced by $t_{0}$ and will define how far the fly moves and the new $t_{1}$ reached in the step. Once a speed and duration are chosen, the direction of movement is selected randomly (in this example to hot). The fly reaches $t_{1}$ resulting in a different probability distribution for velocity and duration that will influence the distance it will move in the next step of the simulation. Modified from Dr. Bart R.H. Geurten. 


\section{II.VI. Genetic dissection of chordotonal larval proprioception}

Genetic tools were used to study role in proprioception of larval chordotonal organs (chos) and different genes expressed in them. Behavioural and physiological experiments were carried out for this purpose and will be described in this section.

\section{II.VI.I. Behavioural characterization of proprioception}

\section{II.VI.I.I. Larval crawling analysis}

Larvae that have proprioception deficits have been shown to have abnormal locomotion (Caldwell et al., 2003). To study proprioception deficiencies, larval locomotion was analysed following the larva crawling assay described in section II.III.I. Larvae were reared at $25^{\circ} \mathrm{C}$ and $60 \%$ humidity in a $12 \mathrm{~h} / 12 \mathrm{~h}$ light-dark cycle. For most experiments, the temperature in the testing incubator was set to $25^{\circ} \mathrm{C}$, except in some cases where the testing temperature was $18^{\circ} \mathrm{C}$ or $29^{\circ} \mathrm{C}$. If the temperature was different from $25^{\circ} \mathrm{C}$ is specified in the relevant sections. Once the larvae locomotion trajectories were obtained, they were smoothed following the protocol established by (Günther et al., 2016). The smoothing of the traces allowed to separate forward crawling points from turning points (Figure 10), and extract features from the locomotion of different strains of larvae.

The traces were smoothed using a threshold $r$, which was empirically calculated as $1 / 3$ of the median length measured for each larva. The algorithm started at the coordinates $\mathrm{x}_{0}$ and time point $t_{0}$, and measured the distance to the coordinates $x_{1}$ at the next time point $t_{1}$. If this distance was lower than $r$, then the algorithm progressed to the next coordinate set $x_{2}$ at time point $t_{2}$. If the distance between $x_{0}$ and $x_{2}$ was smaller than $r$, the algorithm again progressed to the next point. Once the distance between $x_{0}$ and $x_{n}$ was larger than $r$, the average of the coordinates of all the sample points $x_{1 \rightarrow n-1}$ were recorded as the smoothed sample point $x_{\text {smoothed. The point }} x_{n}$ was then used as $x_{0}$ and the algorithm progressed to the next sample points to calculate the rest of the smoothed points. Each smoothed sample point had an $\mathrm{m}$ value assigned that was the number of original sample points used to calculate this smoothed point. See Figure 10A for a diagram of how the smoothing algorithm works.

The average speed of each larva was calculated from the original trace. In addition, the number of turning points above $30^{\circ}$ was quantified. The time to advance one body length was also calculated. This measure was only done for the forward crawling points and does not take into account turning points. Finally, the distance covered between points was measured to quantify how 
persistent the larvae were when crawling forward. All these parameters can be affected by problems in proprioception (Caldwell et al., 2003) and were used to screen for proprioception mutants.

A
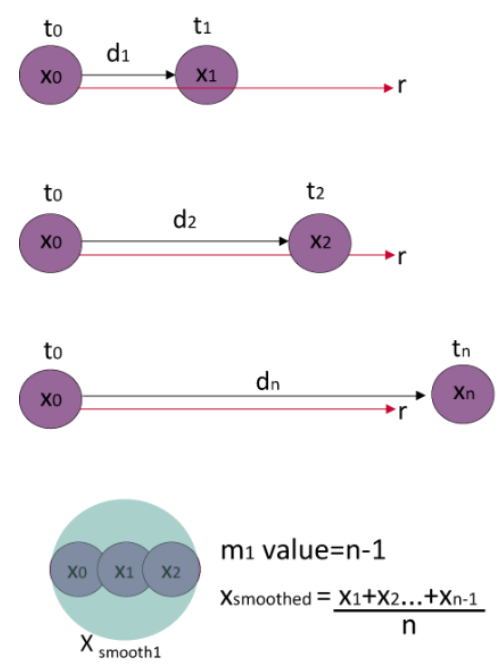

B

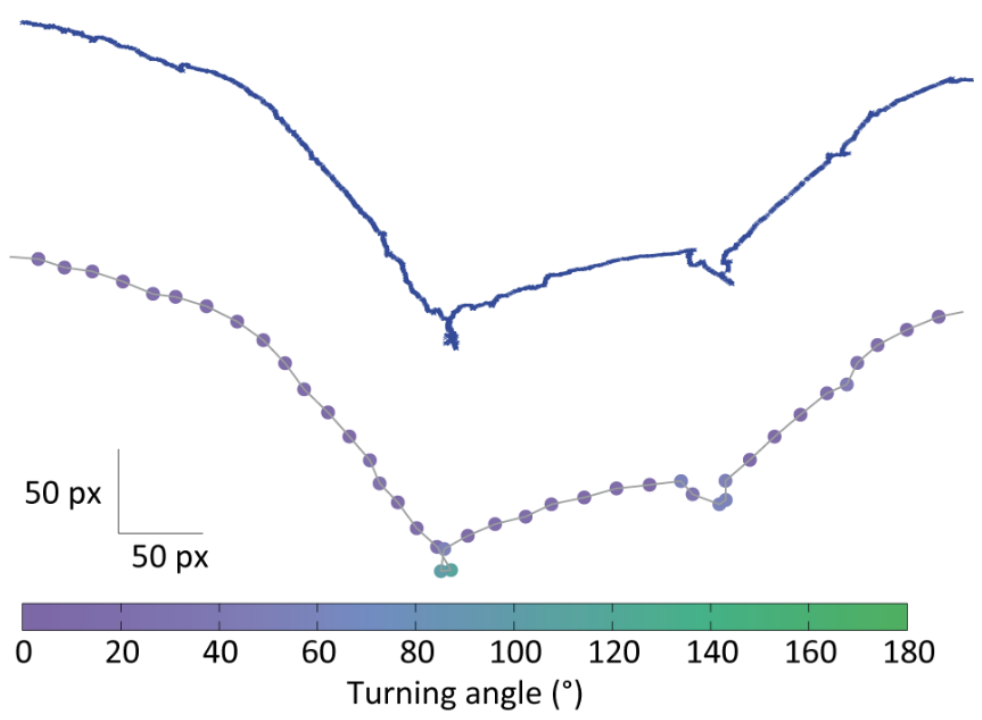

Figure 10. Smoothing of larval crawling traces. A) Diagram explaining the smoothing algorithm after (Günther et al., 2016). $r=$ threshold and $=$ distance moved between points. Once $d_{n}>r$, all the positions from $x_{0}$ to $x_{n-1}$ are averaged to calculate the position of the new smoothed point ( $x_{\text {smoothed }}$ ), and a value of $m$ is given to it. $x_{n}$ then becomes $x_{0}$. The example smoothed point in green would have an $m$ value of 3. B) Example of a smoothed trace. The upper trace shows all the original points traced. The bottom trace shows only the smoothed points. The colours indicate the turning angle that larva did at each point, which allows separating crawling point from turning points.

\section{II.VI.I.II. Larval peristalsis analysis}

\section{II.VI.I.II.I. Mhc-GFP peristalsis analysis}

Lack of proprioceptive feedback can lead to abnormal peristaltic contractions during locomotion (Caldwell et al., 2003; Hughes and Thomas, 2007). To study how peristalsis can be affected the MhcGFP peristalsis assay described in section II.III.II.I was used. This assay allowed to separate the different segments of the larva and study their contraction. The amplitude of segment contraction was indirectly measured by quantifying the change in fluorescence of each segment $(\Delta F / F)$. The higher the amplitude of segment contraction the more GFP molecules come together, which leads to a higher $\Delta \mathrm{F} / \mathrm{F}$ (Figure $6 \mathrm{~A}, \mathrm{~B}$ ). 


\section{II.VI.I.II.II. FTIR peristalsis analysis}

The Mhc-GFP method described in the previous section proved to be an efficient way to measure peristaltic contractions of larvae. However, it required GFP expression in the muscles of every line to be tested. To overcome this problem, FTIR could be used in any line since it does not require the genetic encoding of GFP in the muscles. The assay described in section II.III.II.II was used to study peristalsis in larvae.

The videos were analysed with a custom-made Matlab routine that detected different parts of the larva automatically (Figure 6C). The inner and outer boundary of the larva were detected using an ellipse detection algorithm (Basca et al., 2005; Xie and Ji, 2002) (implemented in Matlab by Martin Simonovsky). To obtain the body axis of the larva, Voronoi cells (Voronoi, 1908) were seeded using the coordinates of the body wall as centres. Then, the Dijkstra (Dijkstra, 1959) path from anterior to posterior end on the inner vertices of the cells was calculated. The body axis would change in length as the larva elongated and contracted (Figure 6D), and these changes reflected the peristaltic contractions during locomotion. The maximum length of the larva was calculated, and the percentage of contraction relative to this length was used to quantify the peristaltic contractions. The program calculated the area under the curve of the body axis and considered a high value for this parameter as a turning point. As a result, the turning points were automatically detected and would be excluded from the analysis, so only peristaltic contractions during forward crawling were analysed (Figure 6D).

\section{II.VI.II. Calcium imaging of larval chordotonal organs}

Mechanically evoked calcium responses of the lateral pentameric chordotonal organ of larvae (Ich5) were measured using calcium imaging. The ultrasensitive calcium sensor GCaMP6f (Chen et al., 2013) was expressed nearly panneuronally using a neuronal synaptobrevin (nSyb-LexA) promoter.

\section{II.VI.II.I. Fillet preparation}

The peripheral nervous system of a larva was exposed using the fillet preparation (Figure 11A). The larva was pinned down on a sylgard square (SYLGARD 184, Sigma-Aldrich, Germany) from the anterior and posterior ends using 0.1 diameter stainless steel insect pins (Minutiens, ENTOMORAVIA - Austerlitz Insect Pins) that were cut down to a length of approximately 2-3mm. Once the larva was pinned down, it was covered with saline solution (see appendix, Table 5). The 


\section{Material and Methods}

larva cuticle was cut longitudinally through the middle between the tracheae. Then, all the internal organs, fat, and trachea were removed to expose the brain and peripheral nervous system. The cuticle was pinned down in four corners to expose the body wall neurons. Finally, the brain was cut out to reduce the muscle contractions during the recording. The sylgard square was dried and quickly transferred to a thin coverslip. Once the sylgard square had attached properly to the coverslip, saline solution with $2 \mathrm{mM}$ of calcium chloride (see appendix, Table 5) was added to the preparation.

\section{II.VI.II.II. Mechanical stimulation and imaging of Ich5 neurons}

The calcium responses to mechanical stimuli were recorded (20X magnification) with an inverted fluorescence microscope (IX73, Olympus, Japan) and a CCD camera (OptiMos, QImaging, Rope, USA) using Micro-Manager (Edelstein et al., 2014). The preparation was illuminated with a mercuryvapour-lamp (U-HGLGPS, Olympus, Japan) and a GFP filter was used for imaging. A piezo actuator (P841.1, Physik Instrumente, Karlsruhe, Germany) with an attached stainless-steel insect pin bent at the tip was used to stimulate the Ich5 neurons mechanically. The piezo actuator was controlled by a digital piezo controller (E-709, Physik Instrumente, Karlsruhe, Germany) that was connected to a CED (CED Micro 1401-3, Cambridge Electronic Design Limited, Cambridge, England). The CED was controlled using Spike 2.7 (Cambridge Electronic Design Limited, Cambridge, England) which ran a custom-made program by Dr. Philipp Jähde that allowed to set the frequency and amplitude of stimulation.

Once an organ was found under the microscope the probe was placed in the previous segment, in front of the organs and parallel to the longitudinal axis of the larva (Figure 11A), and a region of interest (ROI) was selected to image the Ich5 neurons (Figure 11B and appendix Figure 40). One recording consisted of three 5 -second sine wave stimuli at $20 \mathrm{~Hz}$ interspaced by 40 seconds intervals to allow the fluorescence to return back to baseline after each stimulus (Figure 11D). Then, another set of 3 stimuli at the same frequency but with an increased amplitude was given. The recordings were carried out with 30ms exposure at 30fps and stored as individual .tiff images.

\section{II.VI.II.III. Calcium imaging analysis}

The images obtained from the calcium imaging were analysed using FIJI (Schindelin et al., 2012). Image drift was corrected using the template matching plugin (https://sites.google.com/site/qingzongtseng/template-matching-ij-plugin), or, in case template matching did not work, the TurboReg plugin (Thévenaz et al., 1998). Then, an ROI was selected 
around the organs (Figure 11B) and the average intensity of the ROI was calculated for each image to check if there was any decay in the signal due to photobleaching. If photobleaching was apparent, a non-fluorescent section of the image was selected with a ROI and an exponential bleach correction was carried out (see appendix Figure 41). Afterward, an average image of the first ten frames was calculated to obtain the baseline fluorescence $\left(F_{0}\right)$. Next, the difference of each frame with the measured average $\left(\mathrm{F}_{0}\right)$ was calculated to obtain the $\Delta \mathrm{F}$. Finally, the difference was divided by the average image $\left(\mathrm{F}_{0}\right)$ to obtain the $\Delta \mathrm{F} / \mathrm{F}$ image. From this image two different measures were measured by creating ROIs: i) the whole organ response, and ii) dendrite response only. The values were saved in text files and further analysed with Matlab.
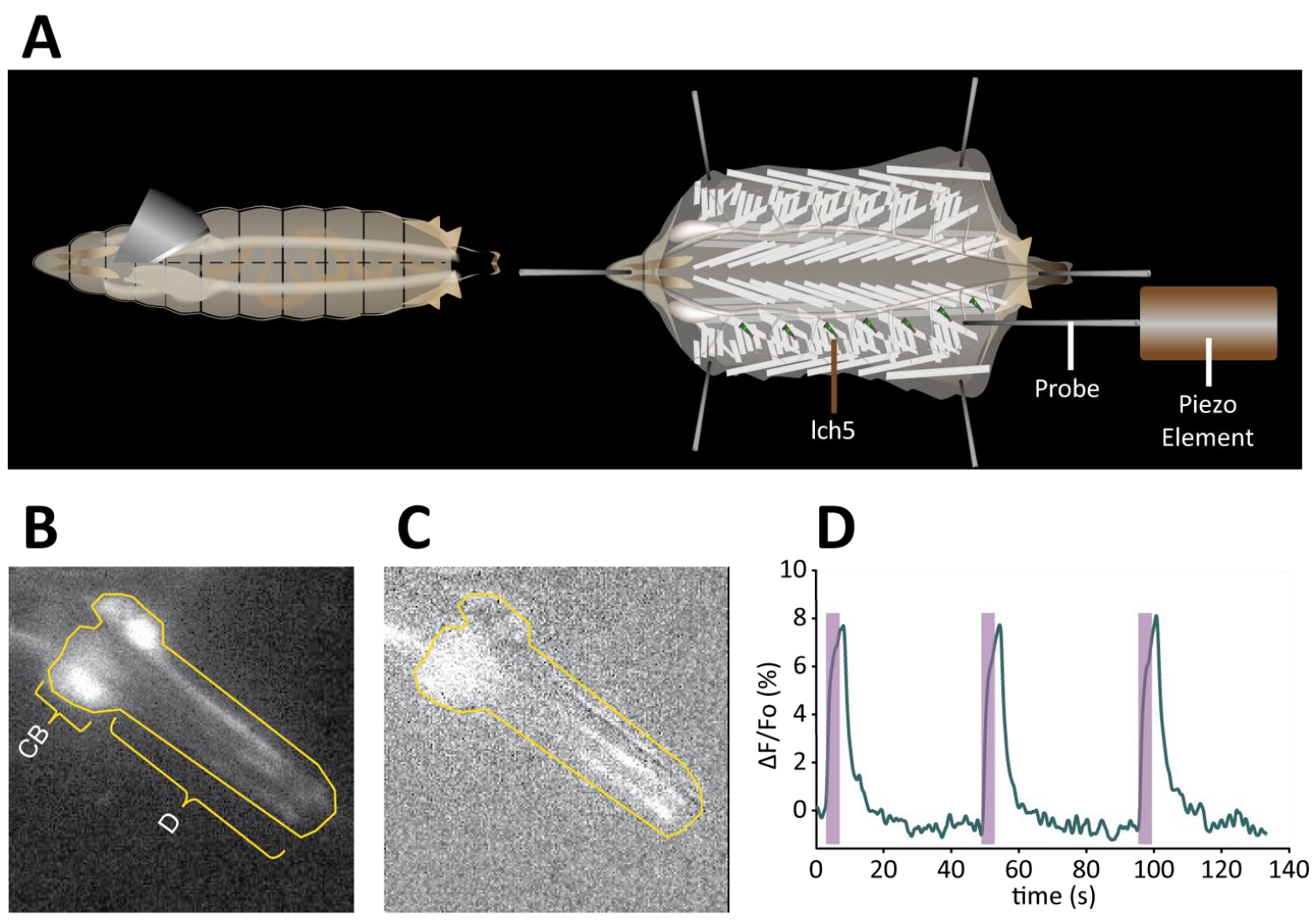

Figure 11. Fillet preparation and calcium imaging of Ich5 organ neurons. A) Larval fillet preparation and mechanical stimulation of Ich5 neurons. The larval peripheral nervous system is dissected by pinning down the larva in the head and tail, cutting the cuticle dorsally through the midline and removing all internal organs, trachea, and brain. The cuticle is pinned down in four corners to expose the body wall neurons. Once an Ich5 is found, a probe attached to a piezo actuator is placed in front of it in the previous segment parallel to the body axis for stimulation. Modified from Dr. Christian Spalthoff and Dr. Bart R. H. Geurten B) Lch5 neurons expressing GCaMP6f. The response of the whole organ or only the dendrites can be calculated by selecting them with regions of interest (ROI). CB: cell bodies, $D$ : dendrites $C) \Delta F / F_{0}$ image of $\mathrm{C}$. The bright pixels show high $\Delta \mathrm{F} / \mathrm{F}_{\mathrm{o}}$ indicating the selected region has calcium influx and the organs are responding to the stimulation. D) $\Delta F / F_{o}$ profile of an Ich 5 stimulated at $20 \mathrm{~Hz}$, and $3 \mu \mathrm{m}$ for 5 seconds (purple bars). The trace shown was smoothed using a Butterworth filter to reduce digital noise. 
The calcium traces recorded were smoothed using a Butterworth filter of two poles to reduce the jitter induced by digital noise (Figure 11D and appendix Figure 41 ). The maximum $\Delta F / F$ of the smoothed trace during the stimulation time was detected. Then, the median value of the 10 points around the maximum $\Delta F / F$ (max value \pm 5 ) of the original non-smoothed trace was obtained to calculate the response to each stimulus. Finally, the response to the three stimuli was averaged to obtain the response of the organ to a certain amplitude.

\section{II.VII. Genetic dissection of larval chordotonal thermosensation}

Genetic tools were also used to study the role of larval chordotonal organs (chos) and different genes expressed in them in thermosensation. Behavioural and physiological experiments were carried out for this purpose and will be described in this section.

\section{II.VII.I. Behavioural characterization temperature preferences}

Larvae were reared at $25^{\circ} \mathrm{C}$ and $60 \%$ humidity in a $12 \mathrm{~h} / 12 \mathrm{~h}$ light-dark cycle. Temperature preferences of larvae were tested using the soldering iron-based assay and the Peltier elementbased assay described in sections II.III.IV.I and II.III.IV.II respectively. In the soldering iron-based assay the gradient generated ranged from $14^{\circ} \mathrm{C}( \pm 1)$ to $30^{\circ} \mathrm{C}( \pm 0.5)$. Once the temperature traces were obtained, a histogram of the distribution around the gradient was calculated for each larva, and an average histogram was calculated for each strain. In the Peltier element-based assay the gradient generated ranged from $12.5^{\circ} \mathrm{C}( \pm 0.5)$ to $28^{\circ} \mathrm{C}( \pm 0.5)$ and a single histogram was determined per strain, calculated from the final distribution of the larvae in the gradient. In both sets of results the data was corrected for cold-trapping as described in section II.V.IV to calculate the preferred and avoided temperatures.

\section{II.VIII. Immunohistochemistry of larval chordotonal organs}

The expression of different proteins in Ich5 neurons was studied. For this purpose, immunohistochemistry and promoter fusion reporters were used.

The primary antibodies used were:

- chicken anti-GFP (GeneTex, Irvine, CA, USA)

The secondary antibodies were:

- Cy3-conjugated goat anti-HRP (Jackson ImmunoResearch, Newmarket, UK)

- Alexa Fluor 488 goat anti-chicken (Thermo Fisher Scientific, Darmstadt, Germany) 
The larval central nervous system was dissected by using the fillet preparation described in section II.VI.II.I. In this case, however, the brain was not removed and the dissecting medium was Phosphate buffered saline (PBS) pH7.4 (Sigma-Aldrich, Germany). After dissection, the preparation was washed three times in PBS pH7.4. The tissue was fixed with $4 \%$ paraformaldehyde in $0.3 \%$ PBSTriton (Triton, Sigma-Aldrich, Germany) for 40 minutes at room temperature, followed by three 20minute PBS pH7.4 washes. The preparation was then incubated in blocking solution (see appendix Table 7) for one hour. Afterward, the blocking solution was removed, and the primary antibodies diluted in the blocking solution were added and incubated overnight at $4^{\circ} \mathrm{C}$. Following the overnight incubation, the preparation was washed five times 20 minutes with $0.1 \%$ PBS-Tween-20 (Tween20, AppliChem, Germany). The secondary antibodies diluted in blocking solution were added and incubated at room temperature for 4 hours. The preparation was once again washed five times 20 minutes with PBST $0.1 \%$ and then mounted on a glass slide using DABCO (Roth, Karlsruhe, Germany). The Ich5 were imaged using a Leica TCS SP8 confocal microscope (Leica Biosystems, Nussloch, Germany) and Fiji was used to analyse the stacks obtained.

\section{II.IX. Transmission electron microscopy of larval chordotonal organs}

The dendritic structure of $3^{\text {rd }}$ instar larvae was studied using electron microscopy. The nervous system of the larvae was dissected using the fillet preparation described in section II.VI.II.I, and icecold $\mathrm{Ca}^{2+}$-free Haemolymph-like HL3 (Stewart et al., 1994) as a medium. The preparation was fixed overnight at $4^{\circ} \mathrm{C}$ with $4 \%$ paraformaldehyde and $2.5 \%$ glutaraldehyde in $0.1 \mathrm{M}$ phosphate buffer with $0.5 \% \mathrm{NaCl}$. Then, three-15 minutes washes in $0.1 \mathrm{M}$ phosphate buffer were done. Postfixation was carried out with $2 \%$ osmium tetroxide $\left(\mathrm{OsO}_{4}\right)$ in $0.1 \mathrm{M}$ phosphate buffer $(\mathrm{pH} 7.4)$ at $4^{\circ} \mathrm{C}$ for 4 hours. Following, the preparation was dehydrated in a serial dilution with acetone: i) $20 \mathrm{~min}$ with $50 \%$ Acetone; ii) $20 \mathrm{~min}$ with $70 \%$ Acetone; iii) $20 \mathrm{~min} 90 \%$ Acetone; iv) $3 \times 20 \mathrm{~min} 100 \%$ Acetone. The preparation was flat embedded in Epon (see appendix Table 6) and polymerised for $24 \mathrm{~h}$ at $60^{\circ} \mathrm{C}$. Ultrathin sections of 70nm were prepared with an ultramicrotome (Leica EM UC7, Leica Biosystems, Nussloch, Germany) and placed on a copper grid coated with 1.3\% Formvar. Contrasting was done by placing the grid 20 minutes in a well with $4 \%$ uranylacetate, then washed with distilled water three times for one minute. Then, the grid was placed in a well containing a $\mathrm{KOH}$ pastille and transferred to a well with lead citrate for 4 minutes. Finally, the preparation was washed 6 times for one minute in distilled water. The ultrathin sections of Ich5 were imaged with a LEO 902 transmission electron microscope (Zeiss, Oberkochen, Germany). 


\section{II.X. Neuronal responses using CaMPARI}

\section{II.X.I. CaMPARI function, set up and imaging}

CaMPARI (calcium-modulated photoactivatable ratiometric integrator) is a genetically encoded calcium sensor (Fosque et al., 2015). CaMPARI has green fluorescence and can be irreversibly converted to red in the presence of UV-light (around 405nm) and calcium (Fosque et al., 2015). Neuronal activity to a certain stimulus can then be measured by quantifying the ratio of red/green fluorescence $\left(F_{\text {red }} / F_{\text {green }}\right)$ (Fosque et al., 2015).

CaMPARI expression was driven in larvae chordotonal organs specifically by crossing a Dhc93AB-Gal4 line with a UAS-CaMPARI line. Third instar larvae were exposed to different stimuli and illuminated with an UV-light LED panel (wavelength 405nm, UV LED Lamp, VCC, Poway, CA, USA). After stimulation, the larvae were dissected using the fillet preparation described in section II.VI.II.I. and mounted on a glass slide in PBS containing 10mM EGTA (the EGTA allows to mount the larvae in low calcium conditions) as suggested by (Fosque et al., 2015). The larvae were covered by a cover slip and fluorescence of the Ich5 was imaged using a Leica TCS SP8 confocal microscope (Leica Biosystems, Nussloch, Germany).

Larvae were imaged using the protocol proposed by (Fosque et al., 2015):

\section{Green CaMPARI:}

Excitation: $488 \mathrm{~nm}$ and $405 \mathrm{~nm}$ laser lines ( $405 \mathrm{~nm}$ line at $5 \%$ the power of the $488 \mathrm{~nm}$ line).

CaMPARI fluorescence under only the $488 \mathrm{~nm}$ laser leads to photoswitching of CaMPARI from a bright state to a dim state (Fosque et al., 2015). Fosque et al. suggest that green CaMPARI is imaged in parallel with a $405 \mathrm{~nm}$ laser at low power to photoswitch it from the dim back to the bright state.

Emission: 495 - 552nm

\section{Red CaMPARI:}

Excitation: $561 \mathrm{~nm}$ laser line

Emission: $570-650 \mathrm{~nm}$ 
Once the images were obtained they were analysed with FIJI. A maximum projection of the stack obtained for each channel (green and red) was calculated and a ROI was selected to obtain the fluorescence from the organs. An additional ROI next to the organs was selected to calculate the background fluorescence of each channel which was subtracted from the measured fluorescence of the organ. Finally, the $F_{\text {red }} / F_{\text {green }}$ ratio was quantified using Matlab.

\section{II.X.II. Chordotonal organ responses to temperature stimuli}

The responses of Ich5 neurons to temperature stimuli was assessed using CaMPARI. Larvae expressing CaMPARI in chos were placed on the Peltier element-based temperature gradient setup described in section II.II.IV. At the beginning of the experiment, all the PEs were kept at a constant temperature of $18^{\circ} \mathrm{C}$. One to two larvae were placed on the arena and the temperature was either lowered, raised, or kept constant (for control experiments) at the same time the UV-light was switched on. The lights were at a distance of $1.5 \mathrm{~cm}$ above the arena and were switched on for one minute while the larvae were exposed to the temperature protocol. The arena was kept in a dark box, so the only light reaching the larvae came from the UV LED's. After one minute the lights were switched off and the larvae were dissected, mounted and imaged as described in the previous section.

\section{II.XI. Statistical Analysis}

Differences between groups were tested using Fisher's exact permutation tests. The p-values were corrected with Benjamini-Hochberg. If a different test was used it will be specified in the relevant section. Unless stated otherwise, in all figures the significances are represented as: $*=p<0.05, * *$ $=p<0.01, * * *=p<0.001, N S=$ not significant. All statistical analyses and graphs were done using Matlab.

For the data represented as boxplots the median is indicated as a black line, the box marks the lower and upper quartile, and the whiskers the range of the entire data set in case there are no outliers. Outliers were detected as any data point outside 1.5 interquartile distance. In case outliers are present the whiskers represent the 1.5 interquartile distance and outliers are indicated by black crosses. 


\section{Chapter 1}

\section{IGLOO - IGLOO is a Gradient}

LOcomotion módel: A model for

\section{temperature-dependent locomotion to correct cold trapping bias}

\subsection{Introduction}

Sensing the environmental temperature is essential for the survival of small ectothermic animals like Drosophila, whose body temperature depends on the environment. Animals not only have to avoid potentially lethal extreme hot or cold temperatures, but also suboptimal temperatures that may be less favourable and reduce their overall fitness. In addition, adult flies and larvae have to pick an optimal temperature for oviposition and pupation since these stages are not motile and cannot escape noxious temperatures (Dillon et al., 2009).

\subsubsection{The anatomy and molecular mechanisms of thermosensation in Drosophila}

Temperature sensation can be divided into four classes: the sensation of innocuous warm temperatures, innocuous cool temperatures, nociceptive hot temperatures, and nociceptive cold temperatures (Barbagallo and Garrity, 2015). Drosophila larvae and adults have specialised thermoreceptors of all four classes that express specific sensory molecules that mediate temperature sensation at different temperature ranges. 


\subsubsection{Larval thermosensors}

The sensation of warm temperatures in larvae is attributed to neurons of the body wall and the brain (Liu et al., 2003; Rosenzweig et al., 2005). Some of these neurons express the heat sensitive channel dTRPA1 (Rosenzweig et al., 2005; Shen et al., 2011), which is activated by temperatures around $25^{\circ} \mathrm{C}$ (Hamada et al., 2008). The sensation of uncomfortable warm temperatures in larvae (i.e. temperatures between $20^{\circ} \mathrm{C}-25^{\circ} \mathrm{C}$ ) is dependent on Rhodopsins 1, 5, and 6 (Shen et al., 2011; Sokabe et al., 2016). These opsins seem to interact with dTRPA1 via a secondary messenger cascade mediated by PLC (Kwon et al., 2008; Shen et al., 2011). Sensation of noxious hot temperatures has been attributed to class IV multidendritic neurons (Hwang et al., 2007; Tracey et al., 2003), which require the TRP channel Painless for proper function (Tracey et al., 2003). Since Painless can be activated by temperatures above $40^{\circ} \mathrm{C}$ it is believed to be a sensor for noxious heat (Sokabe et al., 2008).

Thermosensory neurons in the terminal organ in the head respond to cold temperatures (Liu et al., 2003) and are necessary for cold avoidance (Rosenzweig et al., 2008). The TRPC channels TRP and TRPL, found initially to be involved in photosensation (Montell, 2012; Niemeyer et al., 1996), are essential for cold sensation (Rosenzweig et al., 2008). However, expression in terminal organ neurons was not found, and they do not seem to be activated directly by temperature (Fowler and Montell, 2013; Rosenzweig et al., 2008). Furthermore, there is evidence that three neurons of the dorsal organ (also found in the head next to the terminal organ and containing olfactory receptors), respond to cold temperatures, and are necessary for positive thermotaxis (crawling towards warm temperatures) (Klein et al., 2015). The positive thermotaxis and the responses to cold temperatures of dorsal organ cells seem to be mediated by the ionotropic receptors (IR's) IR21a, IR25a, and IR93a (Knecht et al., 2016; Ni et al., 2016). Class III multidendritic neurons in the body wall also respond to very cold temperatures (around $6^{\circ} \mathrm{C}$ ) and are implicated in noxious cold sensation (Turner et al., 2016). The TRP channels NOMPC, TRPM, and Pkd2 are expressed in these neurons, and a knockdown of expression of these channels leads to an impairment in the behavioural response to noxious cold (Turner et al., 2016). Larval chordotonal organs have also been implicated in the sensation of both cold and hot temperatures with the TRPV channel Inactive (Kwon et al., 2010; Liu et al., 2003), but this will be discussed in more depth in Chapter 3. A summary of all the thermosensory molecules in larvae and their ranges of action is shown in Figure 12. 


\subsubsection{Adult thermosensors}

As in larvae, Drosophila adults have distinct sets of neurons and receptor molecules for hot and cold sensation. One of the main thermosensory organs of the adults is the antenna (Sayeed and Benzer, 1996). Three neurons of the arista in the antenna, respond to warm temperatures and mediate the rapid negative thermotaxis of adults (Gallio et al., 2011; Ni et al., 2013). These neurons express the gustatory receptor GR28BD, which is a channel required for negative thermotaxis and can be activated by warm temperatures (Mishra et al., 2018; Ni et al., 2013). An additional set of neurons in the brain, the anterior cells, are also involved warm sensation and are believed to mediate the temperature preference of adult flies (Hamada et al., 2008; Ni et al., 2013). The sensation of noxious hot temperatures requires the channels Painless and dTRPA1 (Neely et al., 2011; Xu et al., 2006). Which neurons mediate these responses to noxious heat is still not clear, although some evidence points to a role of Painless expressing neurons in the antenna and proboscis (Barbagallo and Garrity, 2015; Neely et al., 2011). An additional TRP channel - Pyrexia - is activated by very hot temperatures, but it is not required for heat nociception (Lee et al., 2005; Neely et al., 2011). This channel, however, seems to be essential for the resistance to noxious temperatures and helps flies avoid heat-induced paralysis (Lee et al., 2005).

In addition to the three hot sensing neurons, the arista also houses three neurons that respond to cold, and are essential for the avoidance of innocuous cold temperatures (Gallio et al., 2011). Furthermore, the third antennal segment, the sacculus, also houses cold sensing neurons (Gallio et al., 2011). Both sets of neurons express three molecules (Brivido 1-3), which are essential for the cold response of the neuron (Gallio et al., 2011). Brivido proteins have sequences that are homologous to TRPP channels, but instead of six transmembrane domains they have ten (Gallio et al., 2011). Because of this higher number of transmembrane domains, it is debated whether they can be considered part of the TRP channel superfamily (Fowler and Montell, 2013). The neural and molecular basis of noxious cold avoidance has not yet been studied (Barbagallo and Garrity, 2015). A summary of all the thermosensory molecules in adults and their ranges of action is shown in Figure 12. 


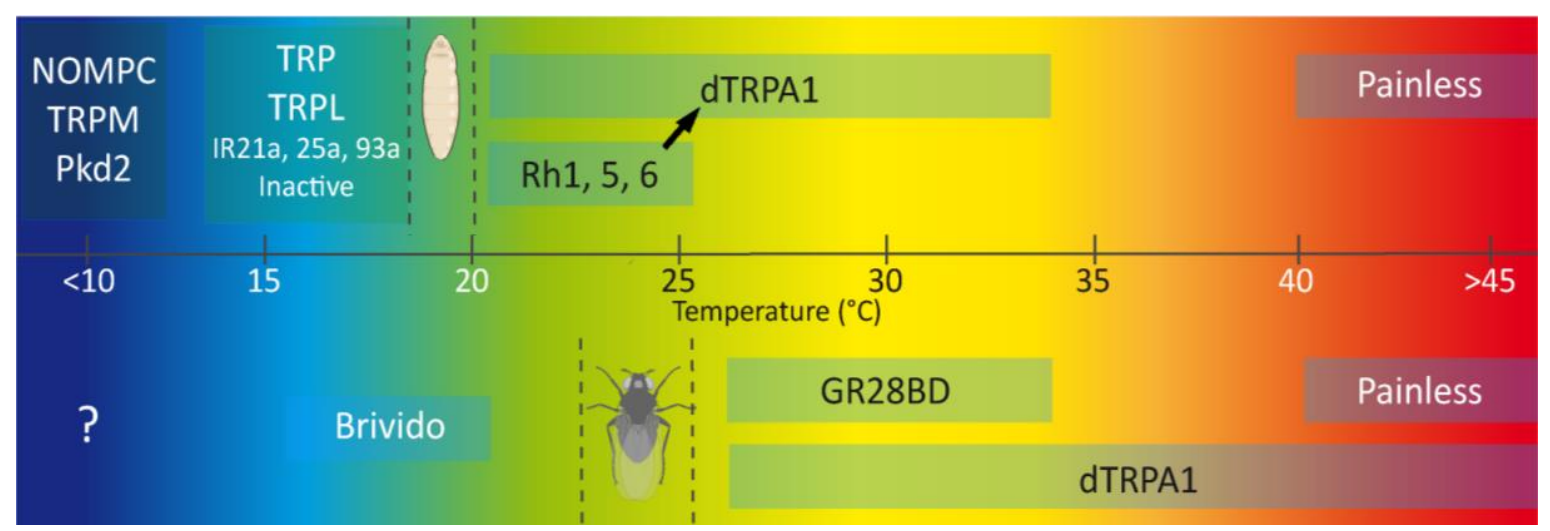

Figure 12. Thermosensory molecules of Drosophila melanogaster. The different receptor molecules involved in thermosensation of larvae (top) and adults (bottom) are placed in the temperature range where they act. The boxes indicate the full range that has been reported for every molecule. The arrow indicates the interaction between Rhodopsins 1, 5, and 6 with dTRPA1 in larvae. The dotted lines indicate the range of preferred temperatures for late third instar larvae and adults. Modified from Andrea Adden, Dr. Christian Spalthoff and Dr. Bart R. H. Geurten.

\subsubsection{Temperature preferences of Drosophila}

Since the body temperature of Drosophila larvae and adults depends on the environment, they tend to prefer temperatures that are optimal for their fitness and survival. Larvae of Drosophila melanogaster have been shown to change their temperature preference from $24^{\circ} \mathrm{C}$ in the first, second and early third instar stages to $18^{\circ} \mathrm{C}$ in the late third instar stage (Figure 12) (Kwon et al., 2008, 2010; Shen et al., 2011; Sokabe et al., 2016). In contrast, adults prefer temperatures between $23^{\circ} \mathrm{C}$ and $25^{\circ} \mathrm{C}$ (Figure 12) (Dillon et al., 2009; Sayeed and Benzer, 1996). The study of temperature preferences allows understanding the neural and molecular basis of thermosensation by testing the effects of removing a certain molecule or groups of neurons on the preference, and thus inferring the range of temperatures they may play a role in (see (Dillon et al., 2009; Fowler and Montell, 2013) for a review).

Two methods for measuring the temperature preferences of larvae and adults have been used extensively: two-choice assays and temperature gradients (Gallio et al., 2011; Hamada et al., 2008; Kwon et al., 2008, 2010; Lee et al., 2005; Ni et al., 2013; Rosenzweig et al., 2005; Sayeed and Benzer, 1996; Sokabe et al., 2016). In two-choice assays, animals have to choose between two temperatures, and preference index is calculated. If the value is close to 0 , the animal has no preference for any of the two temperatures, whereas a value close to 1 or -1 means the animal prefers one of the two. One drawback of two-choice assays is that they only measure the relative preference (i.e., one temperature over the other), but not the absolute temperature preference. To get an idea of the absolute temperature preference of the animal, a comparison between many temperatures would have to be carried out, increasing the number of experiments to be done exponentially. 
Temperature gradients allow the animal to choose between a wide range of temperatures, which resembles the scenario found in nature more than two-choice assays. This significantly reduces the number of comparisons and experiments to be made. The disadvantage of using temperature gradients, however, is that since the metabolic rate and movement of small ectothermic animals depend on temperature, they will suffer a $Q^{10}$ effect in a gradient (Dillon et al., 2009). This results in animals spending more time in the cold part of the gradient, not due to a preference but an inability to move away from those temperatures fast. Additionally, if the temperatures are low enough, the animal can enter a chill coma and stop moving. I will refer to this effect as cold-trapping. As a result, the measured temperature preference may have a cold bias, or the experimenter may recur using only warm temperatures in the gradient (Sokabe et al., 2016).

To avoid the cold-trapping bias one can correct the measured data from a temperature gradient by comparing it to a null model. This null model would represent a fly or larva with no temperature preference and whose locomotion only depends on the ambient temperature. This would result in a null distribution that can then be subtracted from the measured distribution. Such models have been previously proposed for Drosophila (Dillon et al., 2012) and C. elegans (Anderson et al., 2007). These studies used diffusion-based models to simulate their null distributions, which means they simulated groups of animals in a gradient. However, such experiments suffer from group aggregation effects and a positional bias. The positional bias is given by the fact that two or more animals cannot occupy the exact same position in the gradient, as they have a physical extent. Group aggregation is often observed in flies and might lead to a false read-out in gradients. One obvious solution is to isolate animals. For these observations no model exists. In this chapter I describe the generation of such model.

\subsubsection{Chapter overview}

IGLOO (Igloo is a Gradient LOcomotion mOdel), a model that helps correct the cold trapping bias observed in temperature gradient behaviour is described. Instead of using a diffusion based model, IGLOO uses a Random Walk Monte Carlo-type model, which simulates single animals in a gradient, as opposed to groups of animals simulated in diffusion models (Anderson et al., 2007; Dillon et al., 2012). I used IGLOO to correct the temperature preferences of wild-type larvae and adults. In addition, I assessed whether IGLOO can be used as a tool to study the genetics, and understand the neural and molecular basis of thermosensation. 


\subsection{Results}

A model to correct the cold biased temperature preferences derived from cold trapping resulting from chill coma - IGLOO - was created. This model was obtained from the influence of temperature in locomotion of larvae and adults of Drosophila.

\subsubsection{Velocity, bout duration, and activity depend on the local temperature}

The influence of the ambient temperature on the speed of locomotion and bout duration of larvae and adults reared at $18^{\circ} \mathrm{C}$ and $25^{\circ} \mathrm{C}$ (CS18 and $\operatorname{CS} 25$ respectively) was studied. The videos for the adults (CS18 and CS25) were recorded by Andrea Adden (Lund University, Lund, Sweden). A clear influence of the ambient temperature on the median speed was observed (Figure 13A). Larvae and adults show almost no movement at very low temperatures, and the velocity increases with rising temperature. The increase was, as expected, to be much higher for adults than larvae since larvae are much slower than adults; however, the effect in larvae is still very evident. A sharp decrease was observed for adults from around $35^{\circ} \mathrm{C}$, whereas only a slight decrease was seen for larvae after $38^{\circ} \mathrm{C}$. In addition, a big difference was observed between CS18 and CS25 at temperatures between $21^{\circ} \mathrm{C}-29^{\circ} \mathrm{C}$, indicating that rearing temperature does affect the temperature dependence of locomotion. Interestingly, there was peak locomotion speed close to the rearing temperature (Figure 13A), whereas in the case of larvae no clear peak was detected. Furthermore, the decrease in speed happened at slightly colder temperatures in CS25 than CS18 (Figure 13A).

The influence of the ambient temperature on the bout duration (defined as the duration of forward locomotion before stopping for more than 1 second) was less evident. In both adult groups a slight increase was observed with rising temperature (Figure 13B). A drop in the bout duration was only seen in $\mathrm{CS} 18$ at temperatures above $38^{\circ} \mathrm{C}$. The bout duration was more strongly affected in larvae at very low temperatures below $10^{\circ} \mathrm{C}$ (Figure 13B). At these temperatures, larvae had very long bout durations followed by a significant drop at $10^{\circ} \mathrm{C}$. Above $10^{\circ} \mathrm{C}$ there was very little influence of the ambient temperature on the larval bout duration. 
An effect in the activity of the animals was also observed, with all groups being less active at low temperatures and more active at higher temperatures (Figure 13C). A big drop was evident in the activity of both groups of adults around $36^{\circ} \mathrm{C}$. This effect, however, was not apparent for larvae, which showed a considerable increase at $10^{\circ} \mathrm{C}$, and then a steady increase as temperature rises (Figure 13C). Once again, an effect of the rearing temperature was observed, with CS18 being more active than $\operatorname{CS} 25$ at low temperatures (between $9^{\circ} \mathrm{C}-15^{\circ} \mathrm{C}$ ) but less active at higher temperatures (between $19^{\circ} \mathrm{C}-34^{\circ} \mathrm{C}$ ).

A

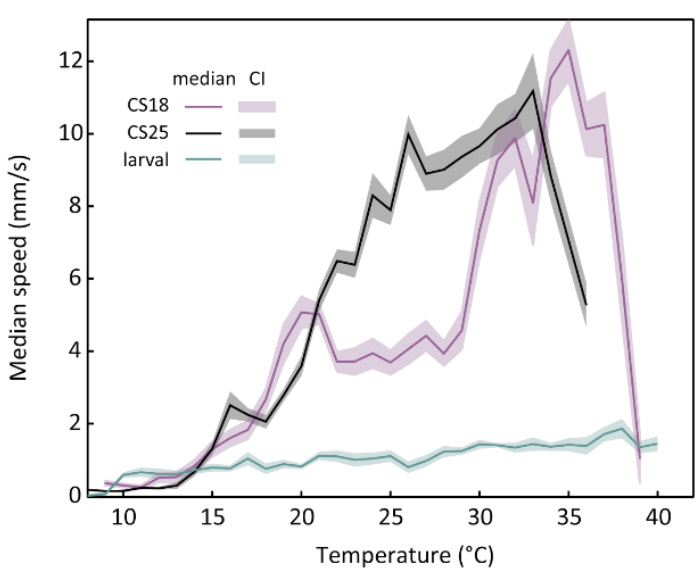

C

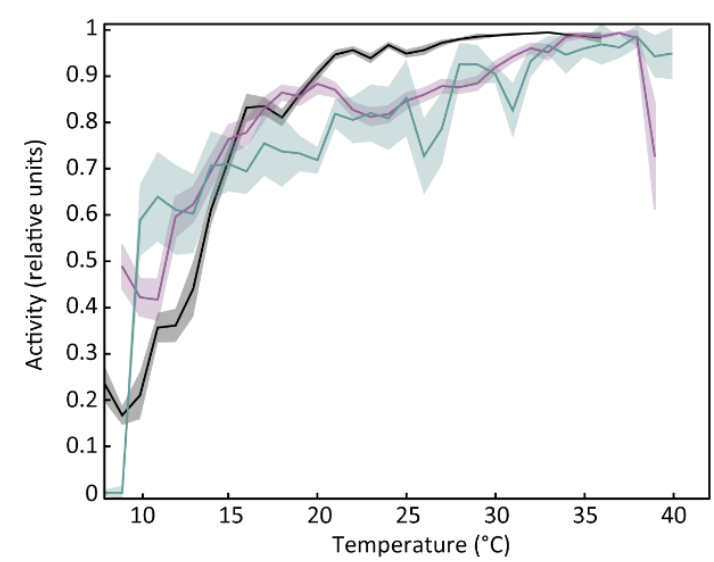

B

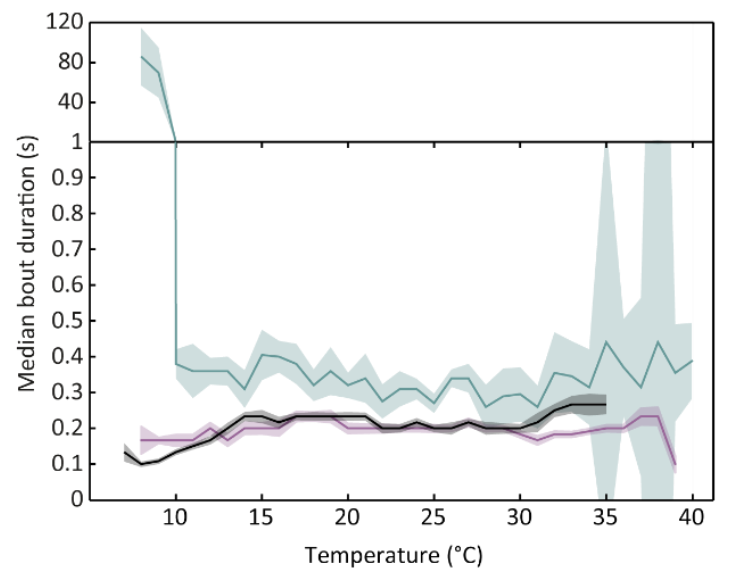

D

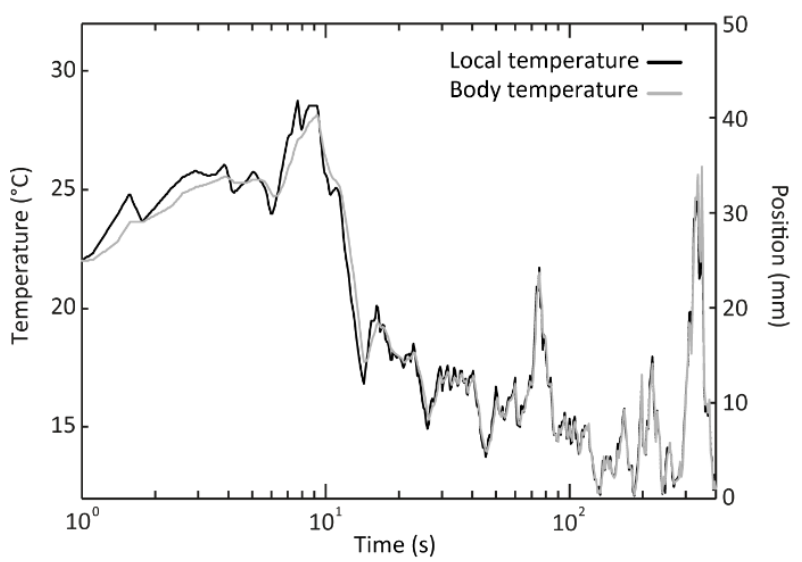

Figure 13. Temperature dependence of locomotion. A) Median speed obtained from the trajectories plotted against temperature for $\mathrm{CS} 18, \mathrm{CS} 25$, and larvae. B) Median bout duration plotted against temperature. Bout duration was calculated as the duration of forward locomotion before stopping for more than 1 second C) Median activity plotted against temperature. Activity was categorized as movement of more than $10 \%$ of the body length of the animal per second. A-C: the solid line represents the median and the shaded area is the $95 \%$ confidence interval. Note: if there is no overlap of the shaded area around the medians the values are significantly different with at least $p<0.05$. D) A trace of an adult fly (CS25) is plotted as a solid black line against the position in the gradient and ambient temperature. The calculated body temperature of the fly given the ambient temperature obtained from Equation 1 is plotted in grey. A-C) $n$ per temperature: larvae $=20, \operatorname{CS} 18=30, \operatorname{CS} 25=30$. 


\subsubsection{Drosophila body thermodynamics}

The actual body temperature of a Drosophila given the local temperature was estimated roughly, assuming that both larvae and adults are a cylinder of water, with a length of $2 \mathrm{~mm}$ and a radius of $0.5 \mathrm{~mm}$, resulting in a surface of $7.85 \mathrm{~mm}^{2}$ (see methods, section II.V.III). The modelled body temperature closely follows the local ambient temperature and seems to low pass filter fluctuations rendered by the random movements through the gradient (Figure 13D).

\subsubsection{Temperature dependence of locomotion fit}

The empirical data obtained on the temperature dependence of locomotion was fitted as follows. For adults, both velocity and bout duration were assumed to be dependent on three parameters: i) $t$ which is the body temperature given the local temperature (see section 1.2.2), ii) $T$ which is the rearing temperature of the animal, and iii) $p$ which is the probability of a certain velocity and bout duration happening given the local temperature.

The velocity function was fitted with a 2-dimensional function based on 2 Gaussian distributions, each with a central temperature $\mu$, a standard deviation $\sigma$, and height $\alpha$ (Figure 14A, C). The probability domain - i.e., how probable a particular velocity or bout duration is given the temperature - was also fitted with a Gaussian distribution (Figure 14A, C) resulting in Equation 2.

Equation 2: $v(p, t, T)=\left(\alpha_{1} e^{-\frac{\left(t-\mu_{1}\right)^{2}}{\left(2 \sigma_{1}\right)^{2}}}+\alpha_{2} e^{-\frac{\left(t-\mu_{2}\right)^{2}}{\left(2 \sigma_{2}\right)^{2}}}\right) * \alpha_{p} e^{-\frac{\left(t-\mu_{p}\right)^{2}}{\left(2 \sigma_{p}\right)^{2}}}$

In the first Gaussian, $\mu_{1}=34^{\circ} \mathrm{C}$ and a $\sigma_{1}=2.27^{\circ} \mathrm{C}$, in the second one $\mu_{2}=26^{\circ} \mathrm{C}$ and $\sigma_{2}=5.41$ ${ }^{\circ} \mathrm{C}$, and in the third one $\mu_{\mathrm{p}}=-1.49{ }^{\circ} \mathrm{C}$ and $\sigma_{\mathrm{p}}=0.5^{\circ} \mathrm{C}$ (Equation 3). The rearing temperature (T) only affected the $\alpha$ factors of each equation. The fits obtained for $\alpha$ were done only using rational or polynomial functions (Equation 3 ) and were limited to $18^{\circ} \mathrm{C} \leq \mathrm{T} \leq 35^{\circ} \mathrm{C}$. Since rearing flies is unlikely to be successful outside of these boundaries this range should cover the most common used rearing temperatures.

Equation 3: $\quad v(p, t, T)=\left(\frac{127.1}{T-17.34} * e^{-\frac{(t-34)^{2}}{(2 * 2.27)^{2}}}+\frac{191.37}{T-16.95} * e^{-\frac{(t-26)^{2}}{(2 * 5.41)^{2}}}\right) *\left(-0.09 T^{2}+4.97 T-\right.$ $58.02) * e^{-\frac{(t-1.49)^{2}}{(2 * 0.5)^{2}}}$ 
The bout duration data could be fitted with the combination of three Gaussian functions as well (Figure 14B, D). In this case, however, only two rearing temperature $(T)$ dependent factors were observed $\left(\sigma_{1}\right.$ and $\left.\mu_{2}\right)$ that could be fitted with a first and second order polynomial (Equation 4). $\mu_{1}=37^{\circ} \mathrm{C}, \sigma_{2}=6.44, \mu_{p}=2$, and $\sigma_{p}=0.64$. Since $\alpha$ no longer depended on $T$, the $\alpha$ values were constant: $\alpha_{1}$ and $\alpha_{2}$ were 20 , and $\alpha_{p}$ was 0.33 . The resulting equation for bout duration was:

Equation 4: $d(p, t, T)=20 *\left(e^{-\frac{(t-37)^{2}}{\left(2 *\left(0.09 * T^{2}-4.76 * T+67.62\right)\right)^{2}}}+e^{-\frac{(t-(1.05 * T-10.42))^{2}}{(2 * 6.44)^{2}}}\right)+0.33 e^{-\frac{(t-2)^{2}}{(2 * 0.64)^{2}}}$

In the case of the larvae, the rearing temperature $(T)$ was not taken into account since only larvae raised at $25^{\circ} \mathrm{C}$ were analysed. Larvae turned out to be more resilient to very cold and hot temperatures than adults (Figure 13A), which resulted in a different fit (Figure 14E, F). The larval velocity was fitted with a fourth-degree polynomial function for the body temperature domain (t) and a third-degree polynomial function for the probability domain (Equation 5). This derived in a steady velocity function that dropped off below $10^{\circ} \mathrm{C}$ (Figure $14 \mathrm{E}, \mathrm{F}$ ).

Equation 5: $\quad v(p, t)=\left(-4.713 t^{4}+167.911 t^{3}-2568.5547+9292.988 t+0.047\right) *$ $\left(-0.002 p^{3}+0.004 p^{2}+-0.001 p+0\right)$

The bout duration of larvae was fitted as the sum of two products of two Gaussian functions (Equation 6), with $\mu_{1}=9, \mu_{2}=34, \mu_{p 1}=0.69$, and $\mu_{p 2}=4.96$. In addition, $\sigma_{1}=0.82, \sigma_{2}=6.79, \sigma_{p 1}=$ 0.12 , and $\sigma_{\mathrm{p} 2}=0.56$ resulting in Equation 7 .

Equation 6: $d(p, t)=\left(8.62 e^{-\frac{(t-\mu 1)^{2}}{(2 * \sigma 1)^{2}}} * 11.74 e^{-\frac{(p-\mu \mathrm{p} 1)^{2}}{(2 * \sigma \mathrm{p} 1)^{2}}}\right)+\left(1860.53 e^{-\frac{(t-\mu 2)^{2}}{(2 * \sigma 2)^{2}}} * 1860.53 e^{-\frac{(p-\mu \mathrm{p} 2)^{2}}{(2 *-\sigma \mathrm{p} 2)^{2}}}\right)$ Equation 7: $d(p, t)=\left(8.62 e^{-\frac{(t-9.0)^{2}}{(2 * 0.82)^{2}} * 11.74 e^{-\frac{(p-0.69)^{2}}{(2 * 0.12)^{2}}}}\right)+\left(1860.53 e^{-\frac{(t-34.0)^{2}}{(2 * 6.79)^{2}}} * 1860.53 e^{-\frac{(p-4.96)^{2}}{(2 *-0.56)^{2}}}\right)$

Interestingly, the $\mu$ values obtained for velocity and bout duration of CS25 and CS18 fit the preferred temperature and the start of noxious hot temperatures for adult wild-type Drosophila (temperatures around $25^{\circ} \mathrm{C}$ and above $34^{\circ} \mathrm{C}$ respectively) (Figure $14 \mathrm{~A}, \mathrm{~B}$ ). This resemblance of naturalistic environmental variables, was not explicitly modelled, but emerged by automatic fitting. Thus showing that the model is able to even replicate naturalistic conditions in the fly which were not set out to model. 


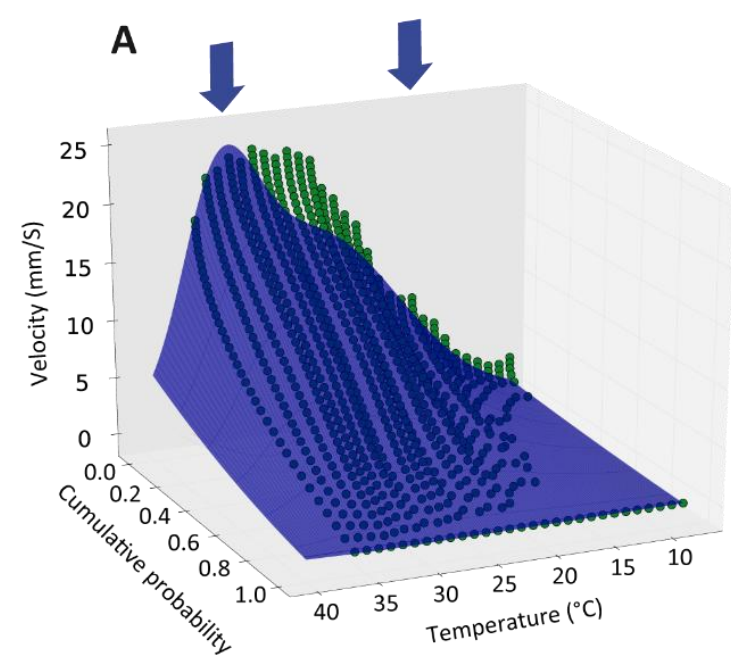

C

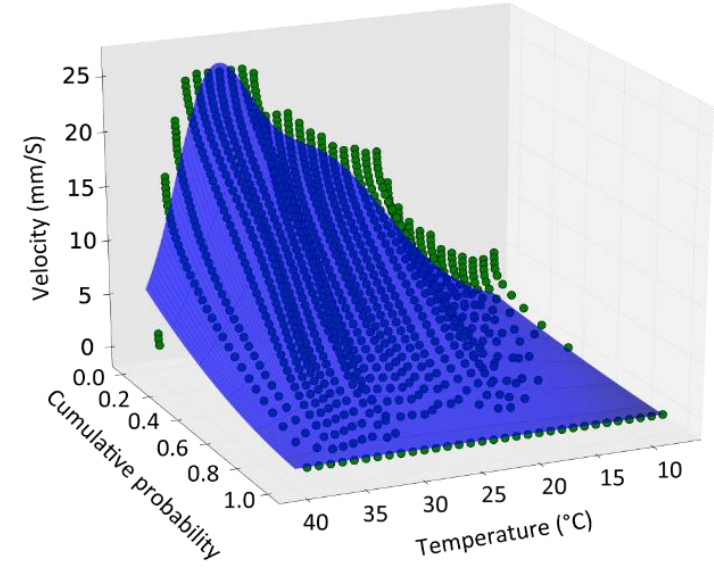

$\mathbf{E}$

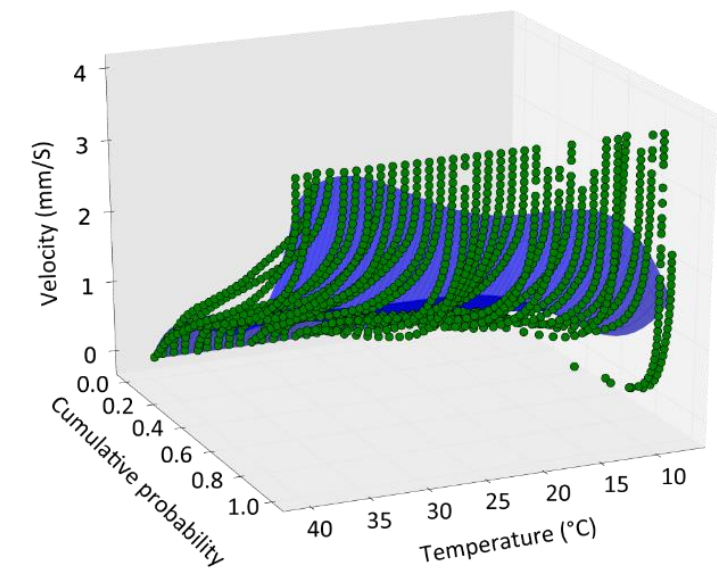

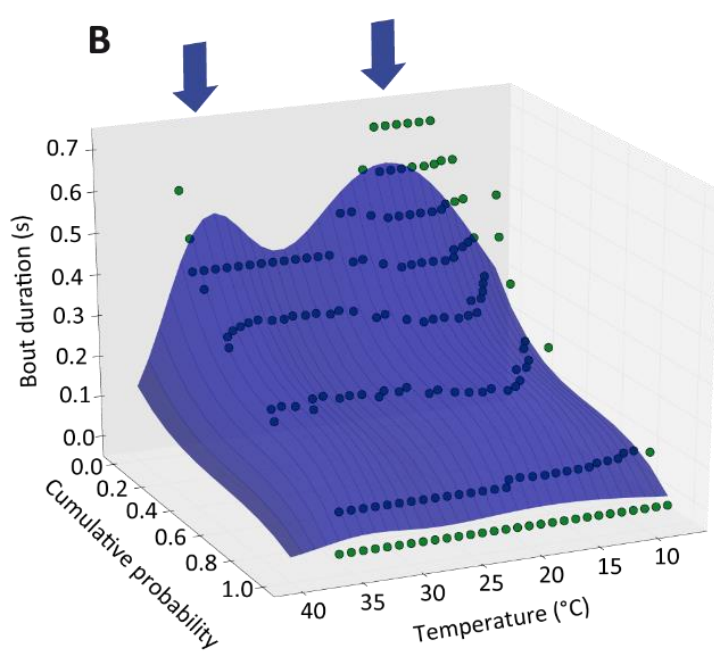

D

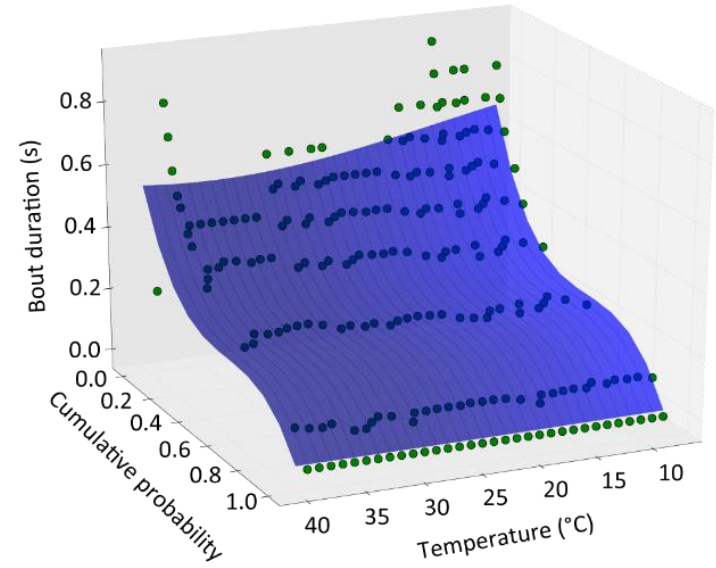

$\mathbf{F}$

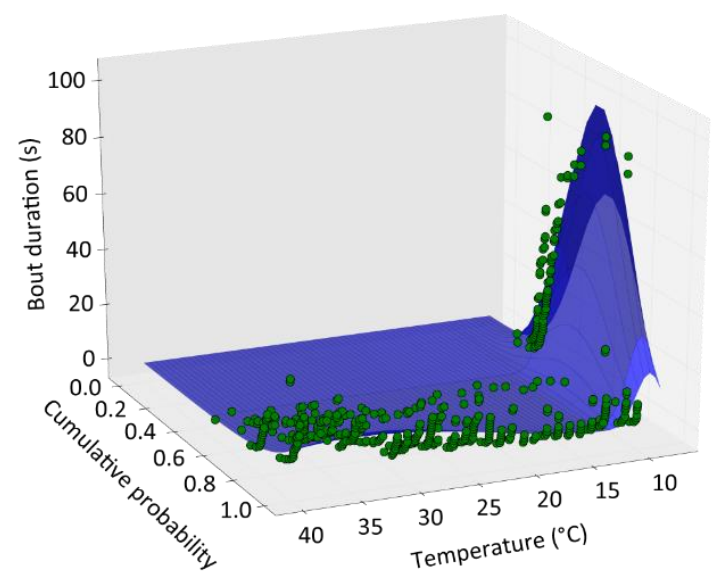

Figure 14. Velocity and bout duration fits. A) Bout duration fit for CS25. The two arrows indicate the peaks of the two Gaussian functions. B) Velocity fit for CS25. The two arrows indicate the peaks of the two Gaussian functions. C) Bout duration fit for CS18. D) Velocity fit for CS18. E) Bout duration fit for larvae. F) Velocity fit for larvae. The $x$ axis represents the temperature, the $y$ axis the velocity or bout durations, and the $z$ axis the cumulative probability of a certain velocity or bout duration happening. Green dots show the fitted data and the fit function is shown in purple. 


\subsubsection{Null model simulation}

The equations obtained from the fits were used to simulate the animal walking around the gradient using Equation 8.

Equation 8: $f(p, t, T)= \pm 1 * v(p, t, T) * d(p, t, T)$

The first component of the equation is the direction of the movement (towards hot or cold), the second component is the velocity, and the third component is the duration of the movement. The velocity and duration were multiplied, which resulted in a distance the simulated animal covered, and a new position with a new local temperature. The final equation for adults was:

Equation 9: $f(p, t, T)= \pm 1 *\left(\frac{127.1}{T-17.34} * e^{-\frac{(t-34)^{2}}{(2 * 2.27)^{2}}}+\frac{191.37}{T-16.95} * e^{-\frac{(t-26)^{2}}{(2 * 5.41)^{2}}}\right) *\left(-0.09 T^{2}+\right.$

$4.97 T-58.02) * e^{-\frac{(t-1.49)^{2}}{(2 * 0.5)^{2}}} * 20 *\left(e^{-\frac{(t-37)^{2}}{\left(2 *\left(0.09 * T^{2}-4.76 * T+67.62\right)\right)^{2}}}+e^{-\frac{(t-(1.05 * T-1.42))^{2}}{(2 * 6.44)^{2}}}\right) *$

$0.33 e^{-\frac{(t-2)^{2}}{(2 * 0.64)^{2}}}$

The final equation for larvae was:

Equation 10: $f(p, t, T)= \pm 1 *\left(-4.713 t^{4}+167.911 t^{3}-2568.5547+9292.988 t+\right.$ $0.047) *\left(-0.002 p^{3}+0.004 p^{2}+-0.001 p+0\right) *\left(\left(8.62 e^{-\frac{(t-9.0)^{2}}{(2 * 0.82)^{2}}} * 11.74 e^{-\frac{(p-0.69)^{2}}{(2 * 0.12)^{2}}}\right)+\right.$ $\left.\left(1860.53 e^{-\frac{(t-34.0)^{2}}{(2 * 6.79)^{2}}} * 1860.53 e^{-\frac{(p-4.96)^{2}}{(2 *-0.56)^{2}}}\right)\right)$

The movement trajectories of 1000 individuals (per group) were simulated for 2 hours with a temporal resolution of $50 \mathrm{~Hz}$ in a $50 \mathrm{~mm}$ long temperature gradient ranging from $14^{\circ} \mathrm{C}$ to $30^{\circ} \mathrm{C}$. The position of each simulated animal was recorded over time and a histogram was calculated. The final position was then used as the null distribution (Figure 15). The simulation was done for each group with four different starting places: i) cold, ii) middle, iii) hot, iv) random (Figure 15). As the simulation advanced the number of flies at the cold end increased until an equilibrium was reached and the proportion of cold-trapped animals stayed constant (Figure 16A). This resulted in a null distribution with a peak in the coldest part of the gradient (Figure 15). The probability of coldtrapping was dependent both on the rearing temperature and the life stage, with larvae reaching equilibrium at $60 \%$, whereas CS18 at around $75 \%$ and CS25 at around 79\% (Figure 16B). The time to reach equilibrium was shorter for adults than larvae, but overall it was fast with all groups 


\section{Chapter 1 - Results}

reaching equilibrium after 10 minutes (Figure 16A). In addition, the starting temperature influenced how fast equilibrium was reached, but not the final proportion of cold-trapped animals (Figure 16A, B). Finally, the distribution of non-cold trapped animals in the rest of the gradient was non-linear and it depended on the rearing temperature and the life stage (Figure 17A and Figure 18A, B).

CS25
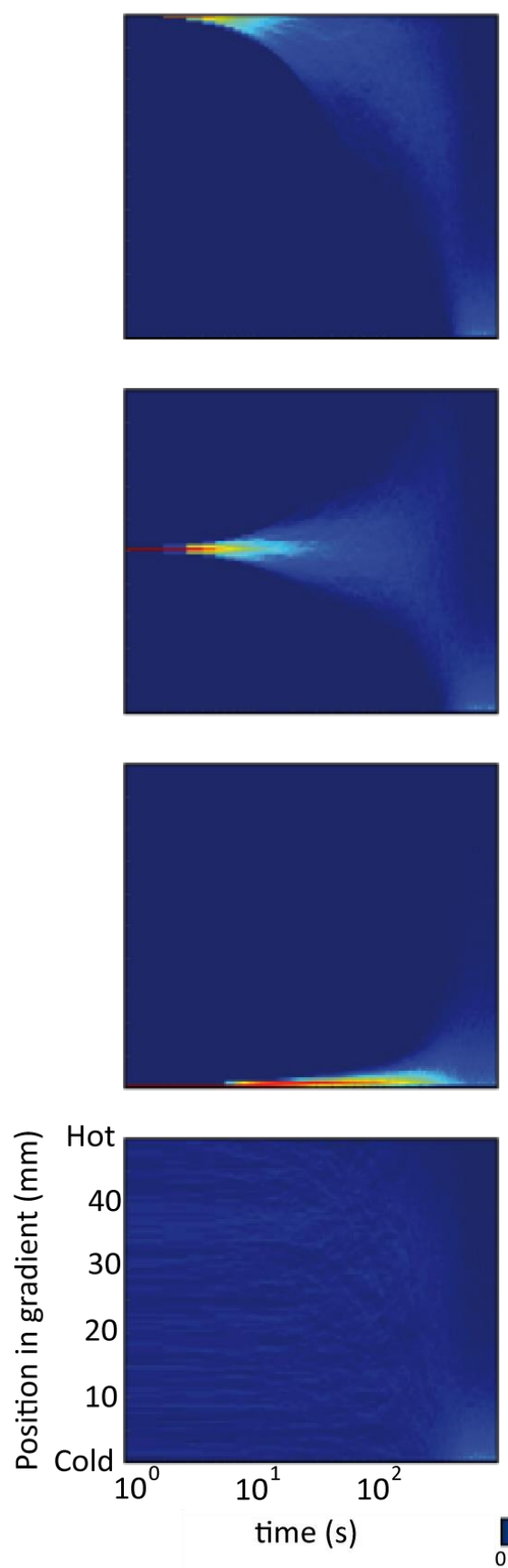

CS18
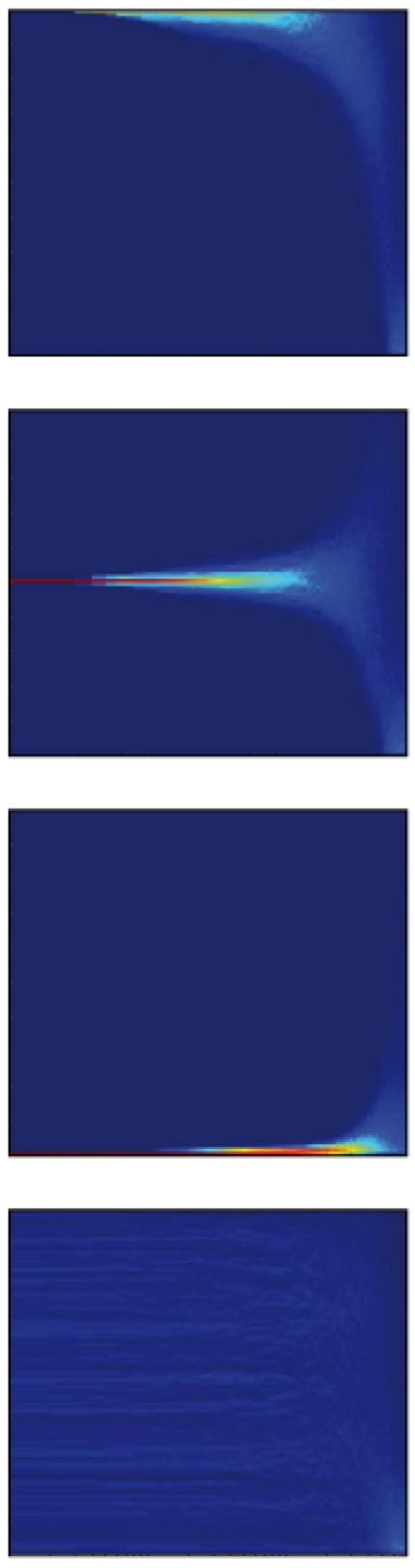

Animal probaility density
Larvae
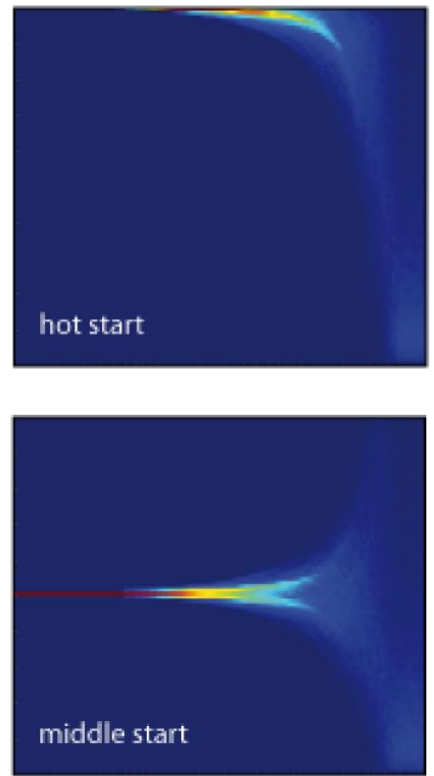

cold start
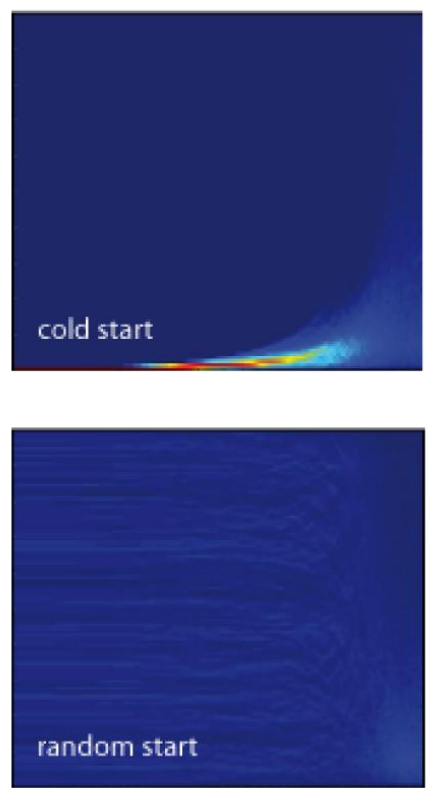

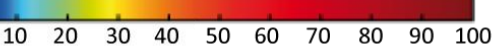

Figure 15. Null distribution simulation. Simulation of 1000 animals of the cohorts (CS25, CS18, and larvae) walking around a temperature gradient for 2 hours. The distribution of animals in the gradient in millimeters is shown through time with four different starting points: hot (first row), middle (second row), cold (third row) and random (fourth row). The time scale ( $x$ axis) is logarithmic. At each time point a histogram of the distribution is plotted in $y$, resulting in one histogram per each time point in $\mathrm{x}$. The distribution after the two hours is taken as the null distribution. Note: the animal probability density scale is non-linear since all animals start at the same point in the gradient in the first three rows. 
A

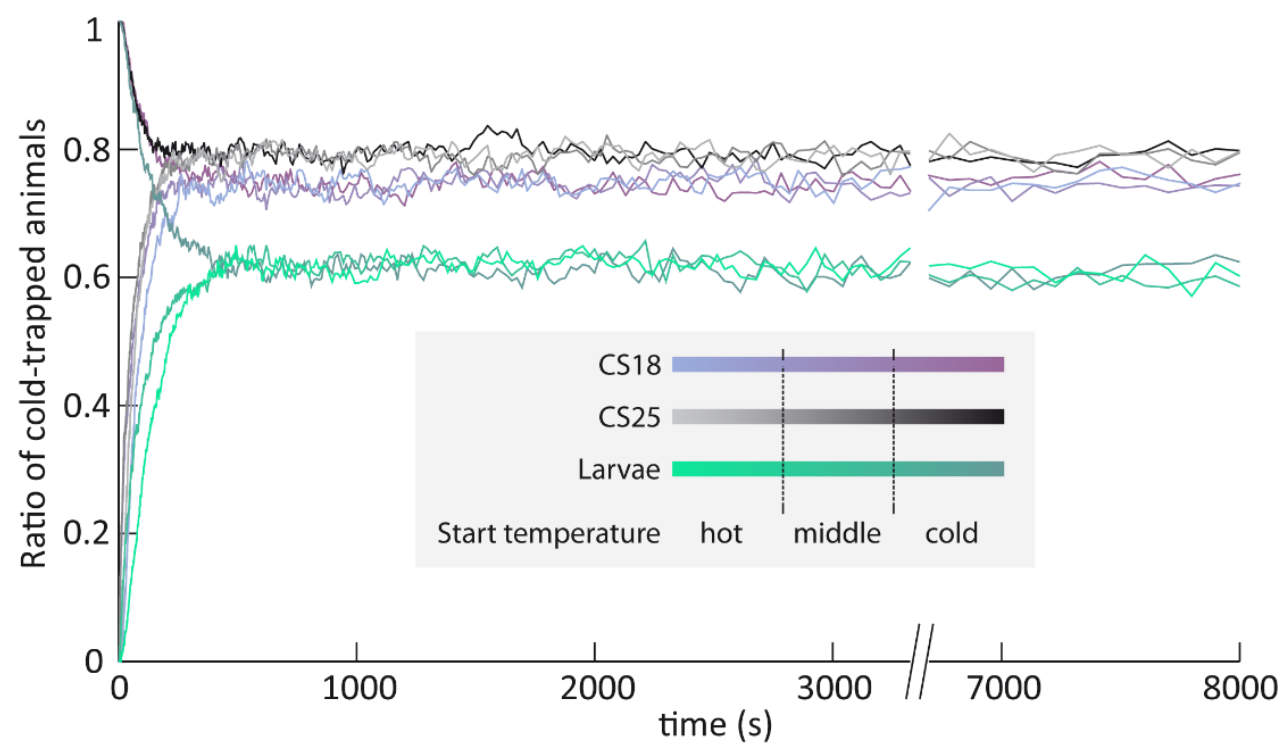

B

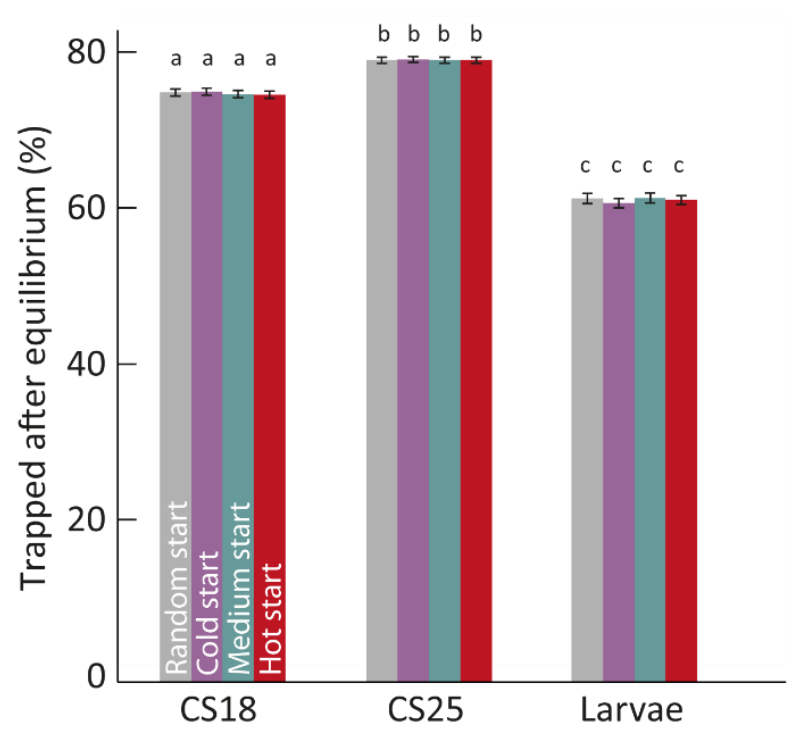

Figure 16. Proportion of cold trapped animals after the simulation. A) Ratio of cold trapped animals through time with three different starting positions: hot, middle, and cold. An equilibrium is reached in all groups in around 10 minutes. B) Median proportion of cold trapped animals after equilibrium is reached with four starting points: random, cold, medium, and hot. 1000 animals were simulated per group and starting point. The bars indicate the median and the error bars the 95\% confidence interval. Significant differences between groups (Rank sum test with Benjamini - Hochberg correction): $a-b: p<0.001, a-c: p<0.001$, b-c: $p<0.001$. 


\subsubsection{Cold trapping bias correction}

To test the model, the temperature preferences of wild-type and mutants with deficits in temperature sensation were tested in a temperature gradient ranging from $14^{\circ} \mathrm{C}$ to $30^{\circ} \mathrm{C}$ using the assays described in sections II.III.IV.I and II.III.V.

\subsubsection{Temperature preference correction of larvae}

Late third instar wild-type Drosophila larvae have been reported to have a temperature preference of $18^{\circ} \mathrm{C}$ (Kwon et al., 2008; Sokabe et al., 2016). Late third instar larvae distributed around the gradient with a peak at $18^{\circ} \mathrm{C}$ and showed a mean preference of $19^{\circ} \mathrm{C}$. The distribution above $24^{\circ} \mathrm{C}$ was very low, however, a distribution below $17^{\circ} \mathrm{C}$ was still observed (Figure 17A). The distribution was corrected by subtracting the null distribution resulting from the simulation of larvae reared at $25^{\circ} \mathrm{C}$ with no temperature preference (Figure 17A). After the correction, the preference index (PI) around these cold temperatures was negative, showing a narrower range of preferred temperatures for larvae between $18^{\circ} \mathrm{C}$ and $20.5^{\circ} \mathrm{C}$. The peak preference remained around $18^{\circ} \mathrm{C}$ and the mean preference was reduced to $18.5^{\circ} \mathrm{C}$ (Figure 17C). The median start of cold avoidance was around $17^{\circ} \mathrm{C}$ and that of hot avoidance around $21^{\circ} \mathrm{C}$ (Figure 17D, E). However, the range of preferred temperatures measured for the entire population of larvae was between $17^{\circ} \mathrm{C}$ and $21^{\circ} \mathrm{C}$ (Figure 17F).

The temperature preference of $d \operatorname{trp} A 1^{\text {ins }}$ mutant larvae lacking the heat sensing TRP channel dTRPA1 (Kwon et al., 2008; Rosenzweig et al., 2005; Shen et al., 2011) was also tested. These larvae did not show an apparent peak in their distribution but spread out along the entire gradient (Figure 17A), including temperatures below $17^{\circ} \mathrm{C}$ that are normally avoided by these mutants (Kwon et al., 2008, 2010). The mean preference was around $21.5^{\circ} \mathrm{C}, 2.5^{\circ} \mathrm{C}$ higher than that of wild-type flies before the correction (Figure 17C). After the correction, however, temperatures below $16^{\circ} \mathrm{C}$ were avoided (Figure 17D) and the mean preference went up to around $23.5^{\circ} \mathrm{C}, 4^{\circ} \mathrm{C}$ higher than the mean preference calculated for wild-type larvae after the correction (Figure 17C). Interestingly, the median start of hot avoidance was around $25^{\circ} \mathrm{C}$, similar to that of wild-type (Figure 17E). However, the range of preferred temperatures starts and ends at higher temperatures than wild-type (Figure 17F), which suggests a shift in the range of preferred temperatures for these larvae. A peak preference was not apparent, which is to be expected from larvae that cannot avoid warm temperatures (Figure 17B). 
A
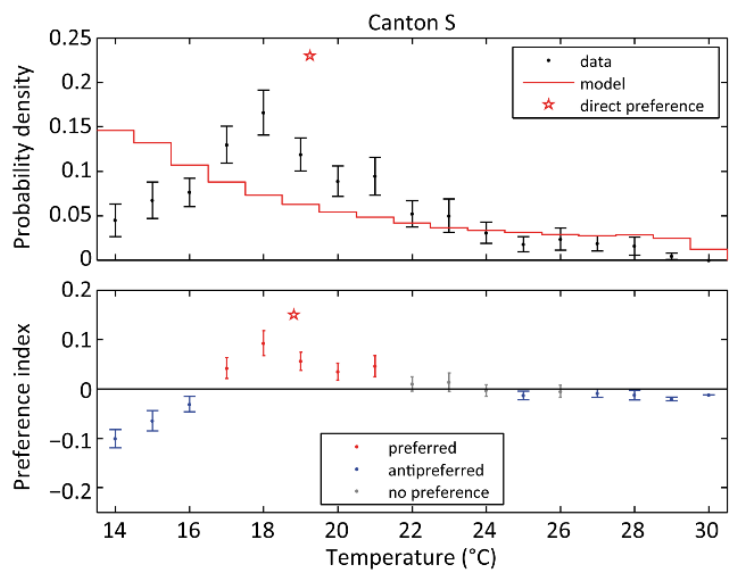

C

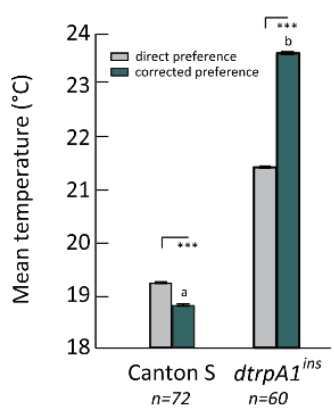

D

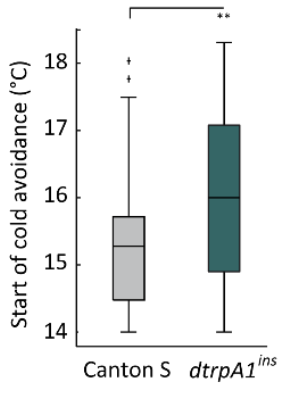

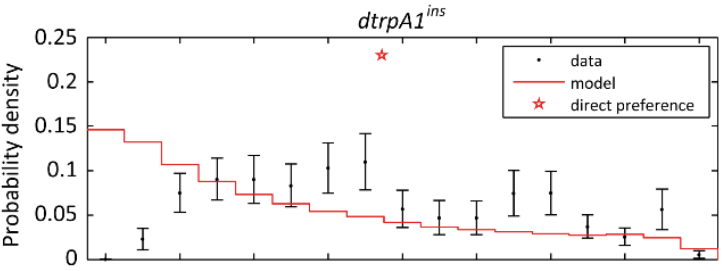

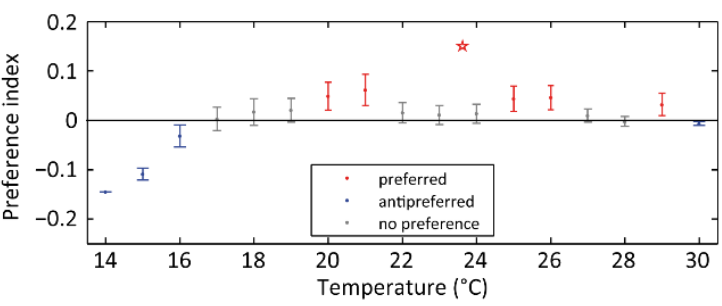

$E$

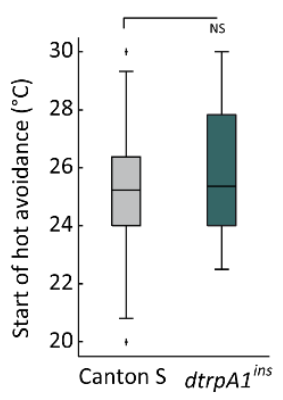

$\mathbf{F}$

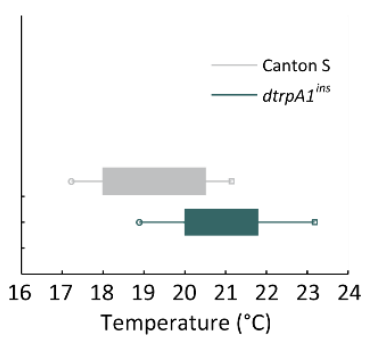

Figure 17. Corrected temperature preferences of larvae. A-B) The uncorrected distribution along a temperature gradient is shown in the top panel in black. The distribution resulting from the simulation is depicted in red. The uncorrected mean preference (direct preference) is indicated as a red star. The bottom panel shows the corrected distribution and preference indices resulting from the subtraction of the simulated distribution from the uncorrected one. The avoided temperatures (negative preference index) are depicted in blue and the preferred temperatures (positive preference index) in red. In grey are depicted the temperatures that are neither preferred nor avoided ( $95 \%$ confidence interval overlaps with a preference index of 0 ). The corrected mean preference is shown as a red star. The points indicate the median and the error bars indicate the $95 \%$ confidence interval. A) CantonS temperature preference distribution. B) $d t r p A 1^{i n s}$ temperature preference distribution. C) Mean temperature preferences before and after correction. The error bars indicate the SEM. D) Start of cold avoidance. E) Start of hot avoidance. D-E: The black line indicates the median, the box marks the lower and upper quartile, and the whiskers the 1.5 interquartile distance; outliers are indicated by black crosses. F) Range of distribution in the temperature gradient. The left edge of the boxes represents the median start of cold avoidance and the error (circle) the $95 \%$ confidence interval of the median. The right edge of the boxes represents the median start of hot avoidance and the error (square) the $95 \%$ confidence interval of the median. Significant differences between groups (Fisher's exact permutation tests with Benjamini - Hochberg correction): $* p<0.05, * *=p$ $<0.01,{ }^{* * *}=p<0.001, \mathrm{NS}=$ not significant. In $\mathrm{C}$ the difference between $\mathrm{a}$ and $\mathrm{b}=\mathrm{p}<0.001$. $\mathrm{n}$ : CantonS72, $d$ trpA $1^{\text {ins }}=60$. 


\subsubsection{Temperature preference correction of adults}

Adults of Drosophila were reared at $18^{\circ} \mathrm{C}$ and $25^{\circ} \mathrm{C}$ (CS18 and $\operatorname{CS} 25$ respectively) and their temperature preference was tested. In contrast to late third instar larvae, adults of Drosophila have been shown to have a preference between $22^{\circ} \mathrm{C}$ to $25^{\circ} \mathrm{C}$. CS25 flies showed a peak distribution around $23^{\circ} \mathrm{C}-24^{\circ} \mathrm{C}$ and a mean temperature preference of around $21^{\circ} \mathrm{C}$ (Figure 18A and Figure 19A). Furthermore, the flies still went to cold temperatures below $18^{\circ} \mathrm{C}$ that are usually avoided. After correction, however, the peak between $23^{\circ} \mathrm{C}$ and $24^{\circ} \mathrm{C}$ remained, and the mean preference went up to $22.5^{\circ} \mathrm{C}$ (Figure $18 \mathrm{~A}$ and Figure $19 \mathrm{~A}$ ). In addition, temperatures below $15.5^{\circ} \mathrm{C}$ and above $27^{\circ} \mathrm{C}$ were avoided (Figure 19C, D), resulting in a much narrower range of distribution between $19^{\circ} \mathrm{C}$ and $24^{\circ} \mathrm{C}$ (Figure 19B). In contrast, CS18 showed a peak at around $18^{\circ} \mathrm{C}-19^{\circ} \mathrm{C}$ but showed a low distribution below $17^{\circ} \mathrm{C}$ (Figure 18B). The mean temperature was around $21^{\circ} \mathrm{C}$ (Figure 19A). After correction, a slight peak was still evident at around $19^{\circ} \mathrm{C}$ and a clear avoidance became apparent for temperatures below $15.5^{\circ} \mathrm{C}$ (Figure 19C). Even though there was not a significant increase in the mean temperature preference, a small increase was seen to around $22.5^{\circ} \mathrm{C}$, similar to what was found for CS25 (Figure 19A). Interestingly, the flies showed a wider range of preferred temperatures than CS25, but this was due to distribution at colder temperatures, suggesting that these flies spend more time in colder temperatures than CS25 (Figure 19B). In both groups, the median start of cold avoidance was around $15.5^{\circ} \mathrm{C}$ and the median start of hot avoidance around $26^{\circ} \mathrm{C}$ (Figure 19C, D).

To study the effects of cold-trapping in mutants that are deficient for heat sensation, temperature preference of flies with ablated heat sensing neurons of the arista ("hot cells", (Gallio et al., 2011)) and dtrpA1 $1^{\text {ins }}$ mutants were studied. For neuronal ablation, the Gal4-UAS system was used to express the apoptotic factors Hid and Reaper (Grether et al., 1995; White et al., 1994) in hot cells. A HC-Gal4 line was crossed with a UAS-hid, rpr line and the progeny was raised at $25^{\circ} \mathrm{C}$. The temperature preference of the offspring resulting from the cross was studied in a gradient. These flies distributed around the gradient evenly, including cold temperatures around $15^{\circ} \mathrm{C}$, and had a mean temperature preference of around $21.5^{\circ} \mathrm{C}$ (Figure $18 \mathrm{C}$ and Figure $19 \mathrm{~A}$ ). After correction, temperatures below $15.5^{\circ} \mathrm{C}$ were avoided and the flies distributed within a much wider range than wild-type flies, between $19^{\circ} \mathrm{C}$ and $28^{\circ} \mathrm{C}$ (Figure $18 \mathrm{C}$ and Figure 19B), with no apparent peak. The mean temperature preference was almost $2^{\circ} \mathrm{C}$ higher than before correction, although the increase was not significant (Figure 19A). A significant increase in the temperature preference was not observed for HC-ablated flies compared to CS25 controls, although a trend towards higher temperature preferences was observed (Figure 19A). Nonetheless, the start of hot avoidance was 
significantly increased (Figure 19D), indicating that these flies did not avoid the warmer temperatures, and distributed at a much wider range (Figure 19B). The start of cold avoidance was not affected in these flies (Figure 19C).
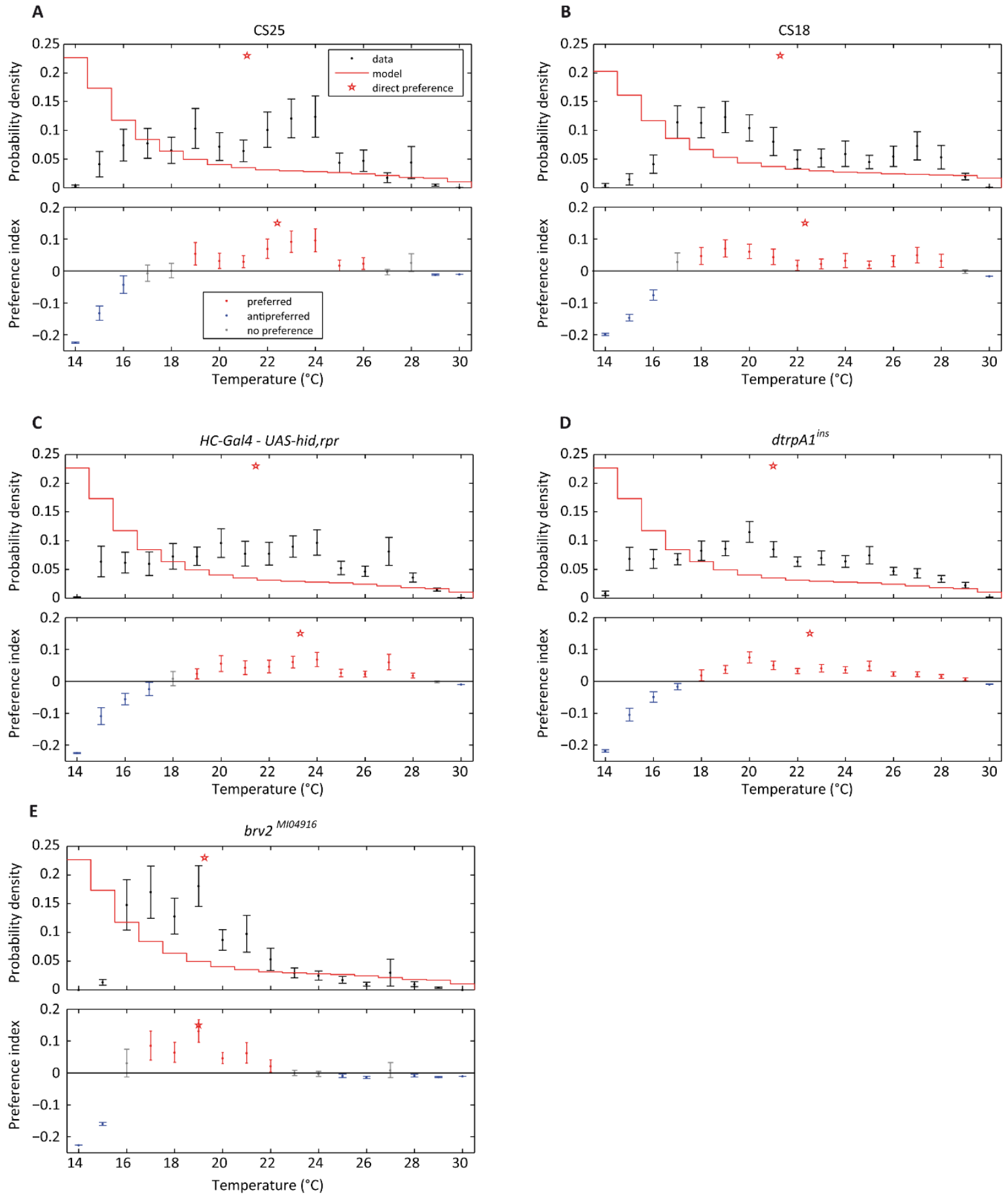

Figure 18. Corrected temperature distributions of adults. A-E) The data is represented as Figure 17A. A) Temperature preference distribution of CantonS adults reared at $25^{\circ} \mathrm{C}$. B) Temperature preference distribution of Cantons adults reared at $18^{\circ} \mathrm{C}$. C) Temperature preference distribution of adults with ablated hot cells. D) Temperature preference distribution of $d \operatorname{trpA} 1^{\text {ins }}$ mutants. E) Temperature preference distribution of brv2 ${ }^{\text {M104916 }}$ mutants. $\mathrm{n}: \mathrm{CS} 25: 45$, $\mathrm{CS} 18=50, H C-G a l 4-U A S-h i d, r p r=40, d t r p A 1^{\text {ins }}=47$, brv2 ${ }^{\text {M104916 }}=37$. 
$d t r p A 1^{\text {ins }}$ mutants also showed an increase in the range of preferred temperatures (Figure 18D and Figure 19B). Like hot cell ablated mutants, these flies distributed at hotter temperatures than the wild-type (Figure 19B). Unexpectedly the flies also distributed at colder temperatures than wild-type flies, and the start of cold avoidance was reduced (Figure 19B, C). No apparent peak was observed after correction (Figure 18D), and the start of hot avoidance was significantly increased compared to the CS25 controls (Figure 19D). Although the mean preference slightly increased after correction, the difference to wild-type was not significant (Figure 19A). This can be explained by the increased distribution around cold temperatures (Figure 19B).

The effects of cold trapping were also studied in cold sensing deficient mutants. For this purpose, the temperature preference of brv2 $2^{\text {M104916 }}$ mutants lacking the channel Brivido2 that has been implicated in cold sensation (Gallio et al., 2011) was studied. In contrast to the heat defective mutants, brv2 ${ }^{\text {MI04916 }}$ mutants did not spread out evenly around the gradient but showed a cold skewed distribution with a peak between $17^{\circ} \mathrm{C}$ and $19^{\circ} \mathrm{C}$ and a mean preferred temperature of around $19.3^{\circ} \mathrm{C}$ (Figure $18 \mathrm{E}$ ). The distribution around the hot temperatures was very low (Figure $18 \mathrm{E})$. After correction, the peaks became less evident but remained at the same temperatures, and only very cold temperatures around $15^{\circ} \mathrm{C}$ were avoided (Figure 18E and Figure 19C). In addition, these flies did not distribute at temperatures higher than $21^{\circ} \mathrm{C}$, although the start of hot avoidance was not affected (Figure 19B, D). Interestingly, the mean preference was reduced after correction to $19^{\circ} \mathrm{C}$ and was significantly lower than that of wild-type adults reared at the same temperature (CS25) (Figure 19A). Additionally, the start of cold avoidance was significantly reduced (Figure 19C). These data indicate that even after correction for cold-trapping the cold phenotype is evident. 


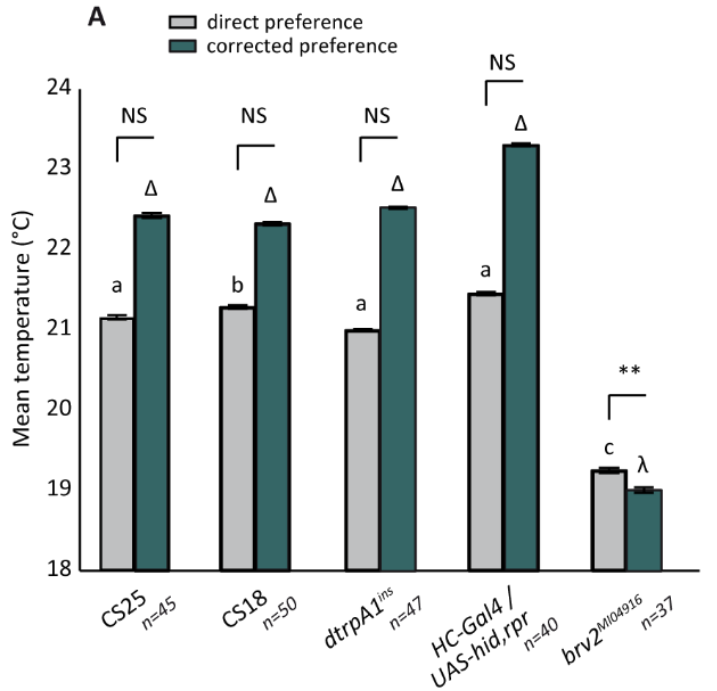

C

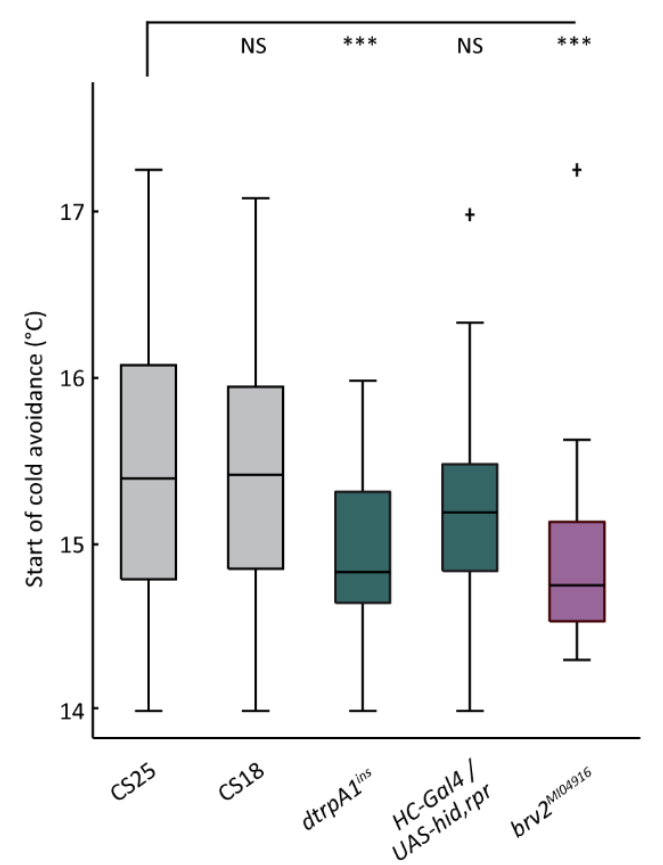

B

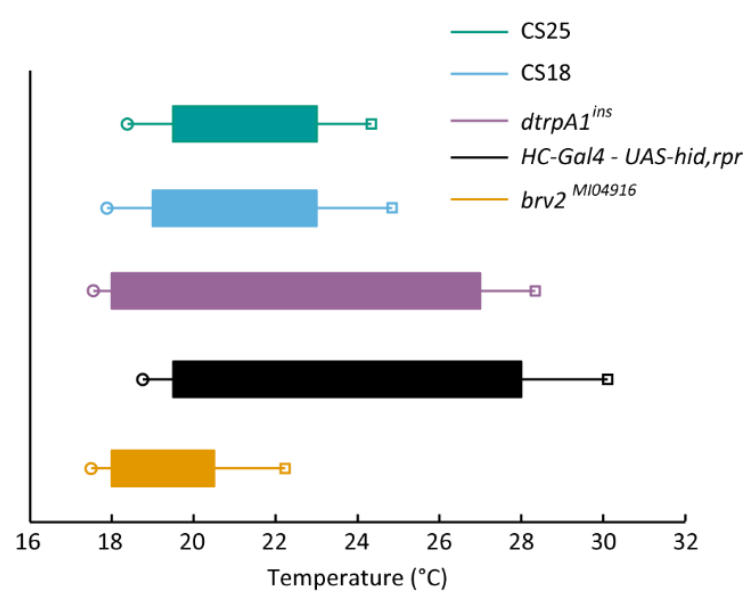

D

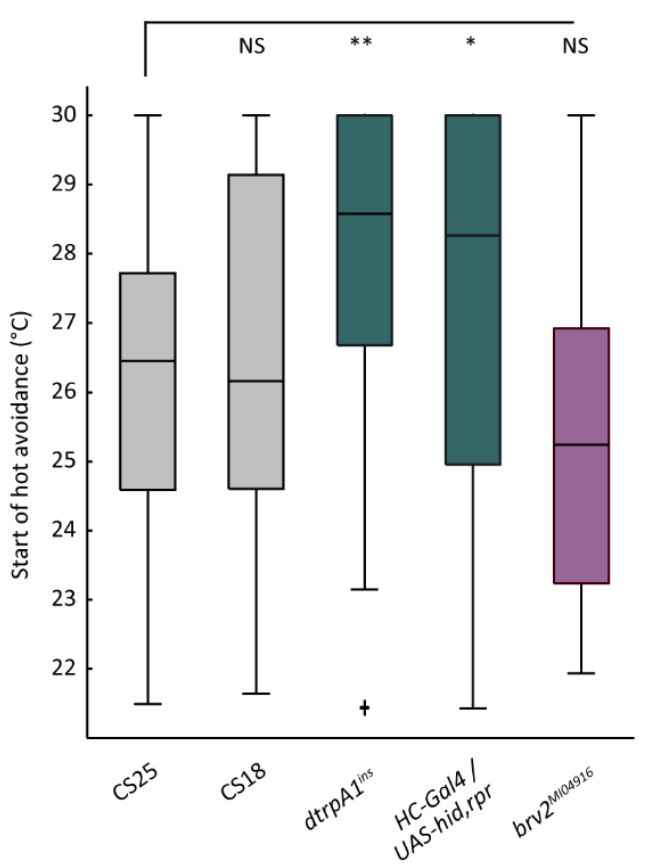

Figure 19. Corrected temperature preferences and start of cold and hot avoidance of adults. A) Corrected mean temperature preferences of adults. The error bars indicate the SEM. Significant differences between groups was calculated with Fisher's exact permutation tests with Benjamini - Hochberg correction. Differences in the mean preferred temperature before and after correction per strain: ${ }^{* *}: p<0.01$, NS $=$ not significant. Differences between CS25 and the rest of the strains before correction depicted as Latin characters $(a, b$, and $c)$ : $a-b=p<0.05, a-c=p<0.01$. . Differences between CS25 and the rest of the strains after correction depicted as Greek characters ( $\Delta$ and $\lambda$ ). $\Delta-\lambda=p<0.05$. B) Range of distribution in the temperature gradient. The data is represented as in Figure 17F. C) Start of cold avoidance. D) Start of hot avoidance. C-D: Significant differences with CS25 (Fisher's exact permutation tests with Benjamini - Hochberg correction): ${ }^{*}=\mathrm{p}<0.05,{ }^{* *}=\mathrm{p}<0.01,{ }^{* * *}=\mathrm{p}<0.001, \mathrm{NS}=$ not significant. The data is represented as in Figure 17D. $\mathrm{n}$ : CS25: 45, CS18 $=50$, HC-Gal4 - UAS-hid, $r p r=40, d$ trpA1 ${ }^{\text {ins }}=47$, brv2 ${ }^{\mathrm{M} 104916}=37$. 


\subsection{Discussion}

The study of behavioural responses of animals to temperatures has become an important tool to understand the molecular mechanisms of temperature sensation (Barbagallo and Garrity, 2015; Fowler and Montell, 2013; Hamada et al., 2008; Kwon et al., 2008; Ni et al., 2013; Rosenzweig et al., 2005, 2008; Shen et al., 2011; Sokabe et al., 2016; Tracey et al., 2003). However, since the ambient temperature has a strong influence on locomotion, and flies may enter a chill coma at very low temperatures (MacMillan and Sinclair, 2011; Mellanby, 1939), experimental results may be biased. IGLOO is model that can verify and extend the understanding of the results obtained in behavioural experiments.

Two models that address the cold trapping due to chill coma bias problem were previously published (Anderson et al., 2007; Dillon et al., 2012). In both, animals in a thermal gradient are simulated using diffusion-based models that result in an overall distribution of animals in a temperature gradient. The resulting null-distribution can then be compared to a distribution obtained experimentally. The drawback of such models is that groups of animals are simulated, which will result in a lower proportion of cold trapped since the cold end cannot have an infinite number of animals. It is therefore essential to model single-fly trajectories for a detailed analysis of the behaviour of individual flies. IGLOO is based on data of walking Drosophila in various temperatures that simulates individual animals walking in a temperature gradient. Furthermore, it takes into account the rearing temperature or the life stage of the animal, which were not considered by Dillon et al. 2012.

\subsubsection{Ambient and rearing temperature influence locomotion}

The ambient temperature strongly influenced the locomotor performance in both larvae and adults. The strength of the influence, however, was much smaller in larvae, which presented an almost linear relation between speed and temperature. An influence on the bout duration was only observed at very low temperatures, probably due to an escape response of the larvae to these noxious temperatures. The difference in the influence on the speed of locomotion could be explained by the fact that larvae move with their whole body, and do not require external appendages for locomotion. Since the rate of heat exchange depends on the volume and surface area (Equation 1), one can expect that the temperature of the larval body wall muscles takes longer to change than the six legs of an adult, which have a higher surface area to volume ratio. This does 
not, however, exclude that the larvae could also have some physiological mechanisms that make them more resistant to changes in the environmental temperatures since they cannot escape noxious temperatures as fast as adults. It has been reported that $1^{\text {st }}$ instar larvae have double the expression of Hsp70 (a heat shock protein that confers thermotolerance of hot temperatures) when exposed to hot temperatures, and tend to be slightly more thermotolerant (Krebs, 1999). In addition, larvae have been shown to be slightly more resistant to extremely cold temperatures than female adults (Jensen et al., 2007). It should be noted, nonetheless, that in studies where thermotolerance is assessed, a prolonged cold or heat shock is presented. In the present study, no prolonged heat shock was given, and a very short cold shock was carried out before the experiments with adults. This would favour more the idea that higher resistance is observed in larvae and happened because the small volume of the adult legs will make the muscles get much colder much faster than the body wall muscles used by larvae for locomotion.

After the simulation, the proportion of cold-trapped larvae was also much lower than adults. This difference can be explained by the higher tolerance to cold observed in the locomotion experiments. In addition, when the experiments were carried out in the gradient to assess temperature preference, larvae never seemed to enter a chill coma like the adults. This does not exclude that there was not a cold bias in the uncorrected larval gradient data since cold temperatures would make larvae much slower, making the correction still necessary. This difference does show that the simulation and model generation have to take into account the life stage of the animals being studied. Furthermore, effects of temperature in locomotion of first and second instar larvae should be assessed, since their reduced size could lead to a different resistance to cold, and therefore a different null distribution should be used to correct for cold-trapping. Recently it was shown that larvae change their temperature preferences in different stages (Sokabe et al., 2016), and therefore IGLOO will be a helpful tool to understand the molecular mechanisms underlying these behavioural changes.

Rearing temperature also influenced the resistance to cold temperatures. Adults reared at $18^{\circ} \mathrm{C}$ (CS18) were more resistant and had higher activity in colder temperatures than adults reared at $25^{\circ} \mathrm{C}$ (CS25), which also resulted in a lower proportion of cold-trapped animals in the CS18 simulation. This is consistent with previous studies that showed that development at low temperatures would reduce the chill coma temperature, and therefore increase the resistance to cold in different Drosophila species (Gibert and Huey, 2001; Hori and Kimura, 1998).

The number and size of cells have been reported to be higher for flies reared at cooler temperatures, resulting in larger body size (Adrian et al., 2016; Jalal et al., 2015). According to 
Bergmann's rule, animals in colder climates tend to be bigger. It is hypothesised that since bigger bodies have a lower surface area to volume ratio, this makes them more resistant to cold temperatures (Blackburn et al., 1999; Ray, 1960). This rule has been proven to hold true even for many small poikilothermic organisms like single-celled algae, nematodes and Drosophila (Ray, 1960). If rearing flies at $18^{\circ} \mathrm{C}$ results in larger flies, CS18 flies would be more resistant to cold temperatures and would also take longer to heat up at warmer temperatures. This could explain why CS18 flies have higher activity and are faster than CS25 at cold temperatures, but as temperature increases above $20^{\circ} \mathrm{C}$ the opposite is observed.

The difference observed between CS18 and CS25 could also be explained by a physiological adaptation of flies to colder temperatures. Development or acclimation to $15^{\circ} \mathrm{C}$ generates an increase in tolerance to colder temperatures by lowering the $\mathrm{Na}^{+}$concentration in the haemolymph, and therefore preventing the loss of haemolymph water caused by $\mathrm{Na}^{+}$imbalance at cold temperatures (MacMillan et al., 2015a). Ion imbalance has been proposed as one of the factors that may generate the cold trapping effects since extracellular and intracellular ion concentrations influence the excitability of muscles and neurons (MacMillan and Sinclair, 2011). Another factor that is influenced by rearing temperatures is the metabolic rate. Flies that are raised at cold temperatures tend to have an increased metabolic rate as a mechanism of compensation for the cold environmental temperature that might lower it (Berrigan and Partridge, 1997). However, since CS25 flies were faster than CS18 flies, an increase in the metabolic rate would explain why these flies are faster and more active in colder temperatures, but not why CS25 are faster at warmer temperatures. If CS18 had an increased metabolic rate, a difference would also be expected at warmer temperatures.

CS18 also showed higher speeds above $24^{\circ} \mathrm{C}$ than CS25, and the drop in speed happened at higher temperatures. Drosophila females that were exposed to cold temperatures had an increase in the expression of Hsp70 which conferred resistance to hot temperatures (Sejerkilde et al., 2003). In addition, expression of other heat shock proteins like Hsp23, Hsp26, and Hsp83 was also found to be increased by exposure to cold temperatures (Qin et al., 2005). However, in that study, flies were exposed to temperatures around $0^{\circ} \mathrm{C}$. Whether exposure to non-lethal cold temperatures like $18^{\circ} \mathrm{C}$ also increases $\mathrm{Hsp70}$ levels and confers higher resistance to heat still has to be studied.

A peak in the median speed was observed at the rearing temperature for CS18 and CS25. These peaks indicate that these animals seem to perform better at their rearing temperature, suggesting some sort of adaptation. This is an example of beneficial adaptation that predicts that acclimation to a given environment will give an advantage to an organism that has acclimated to 
such environment over one that has not (Leroi et al., 1994). Gibert et al. in 2001 studied whether rearing temperature might provide beneficial adaptation in locomotion in Drosophila. As reported here and using a very similar methodology, they found a positive effect of ambient temperature on the locomotion speed but found that flies raised at $25^{\circ} \mathrm{C}$ always had higher walking speeds than $18^{\circ} \mathrm{C}$ flies, showing no beneficial adaptation in this case (Gibert et al., 2001). One main difference between the Gibert et al. study and the present study is that all their test groups were reared at $21^{\circ} \mathrm{C}$ and then allowed to acclimate to $18^{\circ} \mathrm{C}$ or $25^{\circ} \mathrm{C}$. Since the rearing temperature was the same for both groups, the effects mentioned above of cell and body size would be the same. This would mean that the beneficial acclimation observed in the present study comes from exposure to a specific constant temperature throughout the whole life stage (i.e., developmental temperature), whereas the effects observed by Gibert et al. come from exposure only at the adult life stage (i.e., acclimation temperature). Furthermore, these authors tested only adult males, whereas here only female adults were tested, which could also account at least partially for the differences observed. The $\mu$ values obtained for adults fit the preferred and noxious values for wild-type Cantons flies. This emergence was present in both groups of CantonS tested (CS25 and CS18), and shows that the model replicates natural conditions in the fly which were not set out to model.

\subsubsection{Cold-trapping bias is corrected with IGLOO}

\subsubsection{Mean temperature preference}

The simulations carried out predicted that a proportion of animals would be cold-trapped in a gradient in all the groups tested. This was evident in the distribution of the animals across the gradient before correction. Larvae distributed around colder temperatures below $17^{\circ} \mathrm{C}$ and adults distributed around temperatures below $21^{\circ} \mathrm{C}$ that are usually avoided (Barbagallo and Garrity, 2015; Kwon et al., 2010; Sayeed and Benzer, 1996; Shen et al., 2011). After correction, cold temperatures are shown to be avoided by the tested animals, resulting in a narrower range of distribution. In addition, a trend to higher preferred temperatures was observed in both adult groups and are closer to temperature preferences that have been previously reported (Barbagallo and Garrity, 2015; Dillon et al., 2009; Sayeed and Benzer, 1996). This was not the case for wild-type larvae since the original preference was closer to what is reported in the literature (Kwon et al., 2008; Shen et al., 2011). This suggests that the cold-trapping bias is not as strong in larvae as the temperature dependence of locomotion data showed. 


\subsubsection{Range of tolerable temperatures}

From the corrected data, IGLOO not only allows to calculate the mean preferred temperature, but also the range of tolerable temperatures. This range would usually not be able to be distinguished in a gradient experiment. Although the animals will tend to have a peak distribution, the gradient experiments of this study show that the peak might not be very clear, or animals tend to distribute along a wider range of temperatures, and only calculating the mean temperature might obfuscate these observations. Since after correction with IGLOO some temperatures in the gradient have a negative PI that indicate avoidance, a clear range can be determined. Without this, it would be hard to determine what values in the gradient indicate which temperatures can be tolerated and which are being avoided.

This parameter turned out to give more information about the true preference of the animals. Even though the mean temperature can hint at where the animals spent most of their time, this does not indicate how variable the distribution was. The range of tolerable temperatures of larvae is in agreement with what has been reported in previous studies, where larvae tend to strongly avoid temperatures above $22^{\circ} \mathrm{C}$ and below $17^{\circ} \mathrm{C}$ (Kwon et al., 2008; Shen et al., 2011). These data reflect that larvae do not have a single preferred temperature but rather a range of temperatures that are still tolerable. This fact tends to be ignored in two-choice assays since the larva is forced to choose between two temperatures. Another fact ignored in this kind of experiments is the fact that a larva is always in a gradient since it is impossible to obtain two separate absolute temperatures, as there are no thermal isolators ( $2^{\text {nd }}$ law of thermodynamics). For example, if a larva has to choose between $18^{\circ} \mathrm{C}$ and $24^{\circ} \mathrm{C}$, and the larva chooses $18^{\circ} \mathrm{C}$, the space between $18^{\circ} \mathrm{C}$ and $24^{\circ} \mathrm{C}$ also has temperatures ranging from $19^{\circ} \mathrm{C}$ to $23^{\circ} \mathrm{C}$. If the larva is distributed around $20^{\circ} \mathrm{C}$, the final preference obtained is $18^{\circ} \mathrm{C}$, even though the larva preferred $20^{\circ} \mathrm{C}$. The gradient data collected shows that if larvae are exposed to a wide range of temperatures, it will tolerate a much wider range, and not only $18^{\circ} \mathrm{C}$ as it is usually concluded for two-choice assays (Kwon et al., 2008, 2010; Shen et al., 2011).

Adults distributed to temperatures up to $24^{\circ} \mathrm{C}$. In the colder part, however, CS18 flies would distribute to lower temperatures than CS25 flies, even though their mean preferred temperature was the same. This difference does not come from the fact that the CS18 showed less cold-trapping in the simulation, and would, therefore, have a weaker correction since the difference in the distribution is observed before the correction as well. These results indicate a clear effect of rearing temperature in the tolerated temperatures of adult Drosophila. One previous study found that the mean preferred temperature of Drosophila melanogaster was not influenced by the 
rearing temperature (Yamamoto and Obha, 1984). Interestingly, in their study, the mean preferred temperature was similar to the uncorrected mean temperatures of the current study $\left(\sim 21^{\circ} \mathrm{C}\right)$, which indicates that they might have some cold-trapping bias. In addition, the lack of differences observed may come from the fact that they only calculate the preferred temperature, but do not see the distribution. Another study also showed that flies reared at $19^{\circ} \mathrm{C}$ and $25^{\circ} \mathrm{C}$ do not differ in their preference (Krstevska and Hoffmann, 1994). Once again, only preferred temperatures were tested and not the distribution along the gradient. In addition, the preferred temperature found in that study was above $27^{\circ} \mathrm{C}$, which is avoided in this study and other previous studies (Ni et al., 2013; Sayeed and Benzer, 1996), suggesting a possible problem in the methodology and how temperature preferences were assessed. Krstevska and Hoffman found that rearing flies at $28^{\circ} \mathrm{C}$ decreased the preferred temperature in females. However, high rearing temperatures have been shown to have a detrimental effect on locomotion performance, which might have influenced their results. It should also be noted that in both studies group behaviour was used to tests preferences, whereas in the present study individual preferences were assessed.

The fact that CS18 flies distribute around cooler temperatures may be due to their higher resistance to colder temperatures as it was observed in the locomotion data. Several studies have studied the physiological and molecular mechanisms of cold resistance in Drosophila (Burton et al., 1988; Kelty and Lee Jr, 2001; MacMillan et al., 2015a, 2015b; Qin et al., 2005; Sejerkilde et al., 2003) (see Macmillan and Sinclair, 2011 for a review). A recent study showed that cold acclimation generates big changes in the transcriptome and metabolome of flies, and helped identify many candidate genes and metabolic pathways that may confer cold resistance (MacMillan et al., 2016). Even though there is clear evidence that developmental and acclimation temperatures confer resistance to cold, in most of the studies the developmental or acclimation temperatures are much lower than $18^{\circ} \mathrm{C}$. Studies where animals are reared or acclimated to tolerable temperatures are required to determine whether the proteins and metabolic pathways that confer thermotolerance to cold temperatures are also affected by rearing at a cold but tolerable temperature (i.e., $18^{\circ} \mathrm{C}$ ).

\subsubsection{Start of cold and hot avoidance}

Another feature that can be extracted from gradient experiments by using IGLOO is the start of cold and hot avoidance. After subtraction of the null distribution, certain temperatures end up with a negative preference index, which is interpreted as avoidance of such temperatures. If only the original gradient data is analysed it would be hard to determine objectively where to make a cutoff and decide what is a tolerated or avoided temperature. The fact that cold-trapping correction with IGLOO results in negative values gives an objective measure of avoidance. 
The median start of hot avoidance was not significantly different between both wild-type adult groups $\left(24^{\circ} \mathrm{C}\right)$. The start of cold avoidance was also the same, which suggests that although $\mathrm{CS} 18^{\circ} \mathrm{C}$ would distribute towards slightly colder temperatures than $\mathrm{CS} 25$, the overall avoidance of cold temperatures would not differ, and flies would not distribute at temperatures below $17^{\circ} \mathrm{C}$.

Larvae had a start of hot avoidance around $22^{\circ} \mathrm{C}$ and of cold avoidance of $17^{\circ} \mathrm{C}$. These data are similar to what has been reported from two-choice assays (Kwon et al., 2008; Shen et al., 2011). However, in two-choice assays the cold avoidance values tend to be very low, suggesting that there is only a slight preference of $18^{\circ} \mathrm{C}$ versus temperatures below $16^{\circ} \mathrm{C}$. This low avoidance values for cold temperatures could be due to cold trapping and an inability of the larvae to move away from the cold temperature towards $18^{\circ} \mathrm{C}$. IGLOO allows obtaining a clear cut between tolerated and avoided temperatures. The results obtained for wild-type larvae confirm that temperatures below $17^{\circ} \mathrm{C}$ are avoided by larvae since the cold-trapping bias is corrected and a clear value of avoidance is obtained.

\subsubsection{Temperature preferences of hot defective mutant larvae}

dtrpA $1^{\text {ins }}$ mutants had a significant increase in the mean temperature preference compared wildtype, and the range of tolerated temperatures was shifted to warmer temperatures. dTRPA1 in larvae has been implicated in the sensation of temperatures warmer than the preferred $18^{\circ} \mathrm{C}$, which would explain the distribution obtained (Kwon et al., 2008; Rosenzweig et al., 2005; Shen et al., 2011). The shift in the range of distribution would suggest that these larvae avoid the tolerated range of temperatures by wild-type $\left(17^{\circ} \mathrm{C}-20^{\circ} \mathrm{C}\right)$. This shift can be explained by the fact that larvae are inserted in the arena in the hot part. The larvae would escape extreme hot temperatures in the hottest part of the gradient, but once they reach warm temperatures, lack of avoidance to these temperatures due to the mutation would result in the larvae settling at such temperatures and never reaching the lower comfortable range. This would not be the case in wild-type that would keep moving to colder temperatures until they reach the comfortable range. This effect is not seen in adults since they move much faster and have a different exploratory behaviour of the gradient. This effect points out that the starting temperature should be taken into consideration when planning the experiments.

Future experiments should focus on understanding the influence of rearing temperature in larvae, and whether this influence can also affect the results obtained for mutants. In addition, the gradient distribution of earlier larval stages should be studied using a gradient and IGLOO, since 
they have been reported to prefer warmer temperatures than late $3^{\text {rd }}$ instar larvae (Sokabe et al., 2016).

Cold defective mutant larvae were not tested since the strains available ( $\operatorname{trp} p^{1}$ and $\left.\operatorname{trp}\right|^{1}$ ) seemed to have strong locomotion phenotypes and would not crawl in the gradient. Other cold defective mutants like Ir25a, Ir21a, and Ir93a should be tested to assess cold trapping correction in cold deficient larvae.

\subsubsection{Temperature preferences of hot defective mutant adults}

The sensation of hot temperatures in adult Drosophila has been shown to involve different groups of sensory neurons. Three groups of hot sensing cells (hot cells) housed in the base of the arista respond to hot temperatures and express the heat sensing channel GR28B(D) (Gallio et al., 2011; Mishra et al., 2018; Ni et al., 2013). Another group of anterior cells in the brain are also activated by warm temperatures and express the heat sensing TRP channel dTRPA1 (Hamada et al., 2008; Rosenzweig et al., 2005). Temperature preferences of adults with ablated hot cells and dtrpA $1^{\text {ins }}$ mutants were very similar to wild-type adults before correction. However, in the case of hot cell ablated animals, this preference had a slight increase not only compared to the direct preference but also to CS25 controls. This is a clear example of how cold-trapping can obscure a thermosensation deficit and raises the question whether some molecules have been excluded as thermosensors because of a similar effect. On the other hand, $d t r p A 1^{\text {ins }}$ mutants did not show a significant increase in the overall preference.

Looking at the distribution data in the gradient gave more information about the behaviour of the flies. Hot cell ablated flies had a very wide distribution around the gradient towards warmer temperatures up $28^{\circ} \mathrm{C}$, and their start of hot avoidance was significantly increased, showing a clear deficit of hot avoidance in these flies. Ni at al. in 2013 claimed that hot cells in the arista are not involved in the preference of flies to hot temperatures in a gradient, but that their role was only in sensing rapid hot changes in the environment. The data obtained in this study contradicts their findings. One explanation for this is that the conclusions from $\mathrm{Ni}$ et al. study come from studying mutants lacking GR28B(D), but not lacking the hot cells. This suggests that GR28B(D) might be indispensable for determining the distribution of an adult in a gradient, but not the hot cells, and other molecules in them may be playing a role in this behaviour. Besides, in their experiments group behaviour was tested, not individual preferences. Effects of group behaviour biasing the results can therefore not be excluded. Aversion to odours has been shown to be enhanced by larger groups of flies and increased touch (Ramdya et al., 2014). Whether a similar mechanism can influence 
temperature preferences and avoidances still has to be tested. Given that many studies on thermosensation use group behaviour instead of individual behaviour (Dillon et al., 2012; Gallio et al., 2011; Hamada et al., 2008; Ni et al., 2013; Sayeed and Benzer, 1996), and the differences observed between group and individual studies in gradient behaviour, the question whether to test individual preferences over group preferences should be taken into consideration when designing an experiment. The start of cold avoidance was not affected in these flies, confirming that the deficit observed is only coming from the lack of hot cells.

Even though $d \operatorname{trpA} 1^{\text {ins }}$ mutants did not show an increase in the overall temperature preference, a wider distribution than wild-type was observed, which extended to much warmer temperatures, but also surprisingly to slightly colder temperatures. ITRPA1 can be activated by heat, is expressed in heat sensing neurons, and is required for hot avoidance in flies (Hamada et al., 2008; Ni et al., 2013). The corrected results for $d t r p A 1^{\text {ins }}$ mutants confirm the warm distribution of these flies that were previously reported (Hamada et al., 2008; Ni et al., 2013). The cold distribution and reduction in the start of cold avoidance might suggest that the cold-trapping bias was not fully corrected for these mutants since it has never been reported that they have any problems detecting colder temperatures. However, in the original publication by Hamada et al. 2008, it can be observed that the distribution of $d \operatorname{trpA} 1^{\text {ins }}$ around temperatures between $20^{\circ} \mathrm{C}-22^{\circ} \mathrm{C}$ is higher than in the wild-type. The authors pooled the data for temperatures between $18^{\circ} \mathrm{C}$ to $22^{\circ} \mathrm{C}$ to show that the avoidance was not different between mutants and wild-type. This explains why in their case avoidance of cold regions near the preferred regions is not different between groups, but in the present study, $d t r p A 1^{i n s}$ mutants distribute at slightly cooler temperatures. The experiments by Hamada et al. were also group behaviour experiments, which as discussed above might influence their results.

The rearing temperature influenced the distribution along the gradient of wild-type flies. The adults tested were reared at $25^{\circ} \mathrm{C}$ and the effect of $18^{\circ} \mathrm{C}$ rearing was not examined. This should be taken into account when comparing data between different studies. For instance, no information is given on the rearing temperature of $d \operatorname{trp} A 1^{\text {ins }}$ in the study of Hamada et al. 2008, which could also explain partially differences found between studies. Given the influence of rearing temperature in temperature gradient behaviour, it should always be reported in publications to avoid confusion and to allow to compare different results. 
Hot cell ablated adults had a higher median temperature preference than $d \operatorname{trp} A 1^{\text {ins }}$ mutants. Interestingly, an increase in the preference was observed in $d t r p A 1^{\text {ins }}$ larvae. While $\operatorname{drtpA}$ expressing neurons in the adult act as internal sensors (Hamada et al., 2008), dtrpA1 in larvae is present in body wall neurons (Shen et al., 2011), and hot cells of the adult act external sensors (Ni et al., 2013). This difference observed may reflect a difference in the mechanisms of external sensors versus internal sensors. Internal sensors may mediate the range of temperatures the animal tolerates, whereas external sensors the preferred temperatures. This contradicts previous findings that show that internal sensors mediate overall preference (Ni et al., 2013). However, in these studies no cold trapping correction was done, and the preferences were calculated for groups of flies. The differences in methodology may explain the contradicting results found in the present study.

\subsubsection{Temperature preferences of cold defective mutant adults}

The sensation of cold temperatures in adults of Drosophila is mediated by three cold sensing neurons (cold cells) in the arista (Gallio et al., 2011). These cells express three TRPP channels Brivido1-3, which are necessary for the behavioural detection of cold temperatures (Gallio et al., 2011). Temperature preferences of brv $2^{M 104916}$ mutants were tested in a gradient. These mutants showed a decreased temperature preference before and after correction. In addition, the range of tolerable preferences was very narrow and lower than that of wild-type, as well as the start of cold and hot avoidance. These data confirm that brv2 ${ }^{\text {M104916 }}$ mutants have deficient cold temperature sensation.

The fact that a cold phenotype was observed in these mutants even after correction proves that IGLOO will only correct the cold bias of flies with normal cold thermosensation but will not camouflage cold phenotypes in mutants with cold thermosensation deficiencies. IGLOO could be used to confirm if other molecules or sets of neurons previously implicated in cold sensation in adults will still have a cold phenotype, or the results were biased by cold-trapping. The distribution of mutant flies at a colder range than wild-type flies could be due to a higher probability of cold trapping in these animals because of their lack of cold avoidance.

Although several groups of neurons in the arista and sacculus of the adult antenna have been characterised as cold sensors (Gallio et al., 2011), the molecular mechanisms of adult cold sensation are still poorly understood (Fowler and Montell, 2013). Getting rid of the cold trapping bias using IGLOO in a temperature gradient seems likely to facilitate tests of the avoidance of cold temperatures. 


\subsection{Conclusions}

IGLOO is a powerful tool that allows correcting the cold shifted temperature preferences obtained from behavioural experiments in a temperature gradient. It can be used to plan experiments by determining temperature extremes in gradients, building null-hypotheses for a given thermosensation experiment, and planning the optimal length of an experiment to reach equilibrium. Because it integrates information from Drosophila larvae and adults, as well as flies that were reared at different temperatures, it can be applied to a wide range of experimental designs. Taken together, IGLOO is a valuable tool for correcting existing data sets as well as designing future experiments. It furthermore allows to discriminate phenotypes in tolerable range, absolute preference, avoidance as well as the difference of interior and exterior thermosensation phenotypes. This vastly differentiates the phenotypes and observations possible to describe and analyse with thermal preference behaviour assays.

The susceptibility to cold trapping makes the study of the molecular mechanisms of cold sensation very complicated in gradient behaviour. Although two-choice assays have been used extensively to study thermosensation, recent studies favour the use of a gradient. However, these studies restrict the gradient used to avoid cold trapping effects (for example Sokabe et al., 2016). IGLOO not only allows to use temperature gradients because of cold-trapping correction but also enables to obtain more information from them, like the range of tolerated temperatures, the start of cold avoidance and the start of hot avoidance. It is computationally sparse and it can be easily modified to apply to other poikilothermic species. 


\section{Chapter 2}

\section{Proprioceptive functions of opsins in Drosophila larval chordotonal organs}

\subsection{Introduction}

\subsubsection{Drosophila opsins and visual transduction}

Opsins are proteins with seven transmembrane domains that belong to the superfamily of Gprotein coupled receptors (GPCR), and most are involved in light sensation in animals (Terakita, 2005). Although the relationship between different subfamilies of visual opsins in neuralia (a monophyletic group containing cnidarians, ctenophore, and bilaterians) is still not clear, they have been divided into three main subfamilies: C-opsins, R-opsins and RGR/Go (retinal G protein-coupled receptor, go-coupled plus retinochrome) opsins (Feuda et al., 2012). C-opsins are found in ciliary cells, whereas R-opsins are found in rhabdomeric cells (Feuda et al., 2012; Yau and Hardie, 2009). All the photoreceptors in Drosophila only express R-opsins (Montell, 2012; Sprecher et al., 2007).

For light detection, opsins bind a light-sensitive chromophore, which in the case of Drosophila is 3-hydroxy 11-cis-retinal to form a functional Rhodopsin (Rh) (Yau and Hardie, 2009). The fly has 7 Rhodopsins (Rh1-7); Rh1-6 are involved in vision, and Rh7 in circadian rhythms (Montell, 2012; Ni et al., 2017). The compound eye of a fly is made of many ommatidia, each with eight rhabdomeric photoreceptor cells (R1-8). Rh1, encoded by the gene ninaE (neither inactivation nor afterpotential E), is the major opsin found in Drosophila and is expressed in R1-6 (O'Tousa et al., 1985; Zuker et al., 1985). R7 expresses Rh3 and Rh4 (Montell et al., 1987; Zuker et al., 1987), and R8 expresses Rh5 and Rh6 (Chou et al., 1996; Huber et al., 1997; Papatsenko et al., 1997). Additionally, two opsins are expressed outside the compound eye; Rh2 is expressed in the ocelli (Pollock and Benzer, 1988), whereas Rh7 is expressed in neurons in the brain (Ni et al., 2017). The larval photoreceptor organ, the Bolwig's organ, expresses Rh5 and Rh6 (Sprecher et al., 2007). 
The light-sensitive chromophore that bind to Rh has to be synthesised from dietary $\beta$ carotenoids that are transported from the gut to extra-retinal neural cells in the head, then to the retinal pigment cells, and finally to the photoreceptors where $\mathrm{Rh}$ is synthesised (Pak et al., 2012; Wang et al., 2007). In different steps the $\beta$-carotene is transformed into the final form 11-cis 3hydroxy retinal required for phototransduction (Wang et al., 2007). Although many of the enzymes needed for the transformation are unknown, NINAB and NINAG mediate parts of the transformations (Sarfare et al., 2005; Wang et al., 2007). In addition, NINAD transports $\beta$-carotene into the gut cells, and SANTA-MARIA, encoded by the gene santa-maria (scavenger receptor acting in neural tissue and majority of rhodopsin is absent), transports it into the extra-retinal cells where it is transformed to all-trans retinal by NINAB (von Lintig et al., 2001; Pak et al., 2012; Wang et al., 2007). Mutations in the genes encoding these proteins lead to deficient phototransduction (Pak et al., 2012).

Phototransduction starts when a photon of light is absorbed by the chromophore which leads to its isomerisation from 11-cis 3-hydroxy retinal to all-trans 3-hydroxy retinal generating Metarhodopsin (Figure 20) (Montell, 2012; Yau and Hardie, 2009). The isomerisation of Rh activates the heterotrimeric Gq-protein by a GDP-GTP exchange in the $\alpha$ subunit of the Gq protein (Hardie, 2001; Scott et al., 1995; Wang and Montell, 2007). This leads to an activation of phospholipase C (PLC), which is encoded by the gene norpA (no receptor potential A) (Bloomquist et al., 1988). The activation of PLC leads to the hydrolysis of phosphatidylinositol 4,5-bisphosphate $\left(\mathrm{PIP}_{2}\right)$ into inositol trisphosphate $\left(\mathrm{IP}_{3}\right)$, diacylglycerol (DAG), and $\mathrm{H}^{+}$(Montell, 2012). PIP 2 hydrolysis is believed to generate a mechanical force in the membrane, which leads to the opening of the TRPC channels TRP and TRPL, and the end of the signal transduction pathway (Figure 20) (Hardie, 2001; Hardie and Franze, 2012; Montell, 2012). A second photon can transform the Metarhodopsin back to Rhodopsin to stop the signalling cascade.

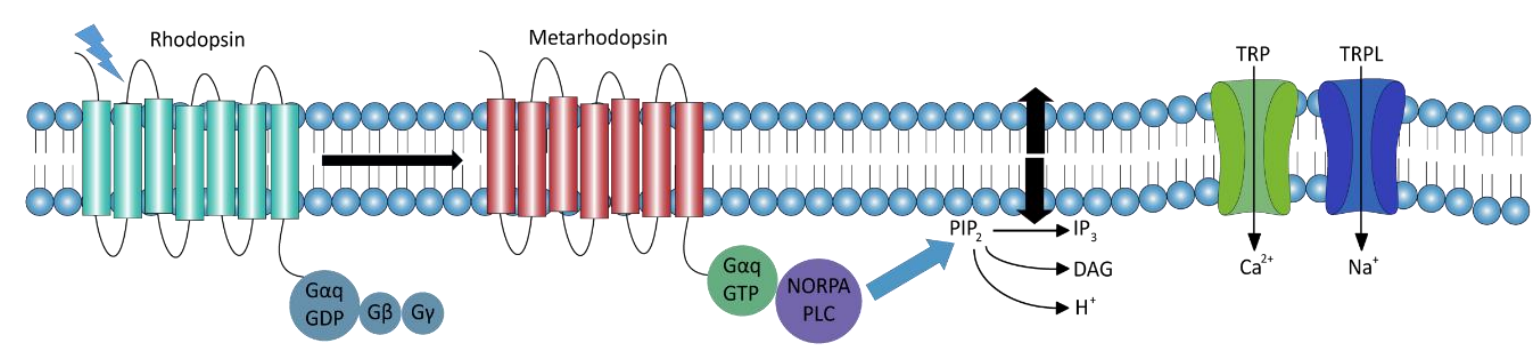

Figure 20. Phototransduction cascade in Drosophila. A schematic of the phototransduction cascade after Hardie and Franze, 2012. One photon of light is captured by the retinal chromophore which converts Rhodopsin to Metarhodopsin. This change activates the $\alpha$ subunit of the Gq protein by a GDP to GTP exchange which in turns activates PLC. PLC hydrolyses $\mathrm{PIP}_{2}$ in the membrane to $\mathrm{IP}_{3}, \mathrm{DAG}$, and $\mathrm{H}^{+}$. This hydrolysis generates a mechanical movement that leads to the opening of TRP and TRPL channels. 


\subsubsection{Opsins in other senses}

Recently, evidence has accumulated showing that opsins are not only involved in photosensation (Leung and Montell, 2017). In Drosophila larvae, Rh1 was found to be needed for the sensation of uncomfortable warm temperatures (Shen et al., 2011). Late third instar Drosophila larvae that have to choose between $18^{\circ} \mathrm{C}$ and any other temperature tend to prefer $18^{\circ} \mathrm{C}$ and avoid warmer and colder temperatures (Kwon et al., 2008, 2010; Shen et al., 2011; Sokabe et al., 2016). However, ninaE mutants that lack Rh1 did not avoid temperatures between $20^{\circ} \mathrm{C}-24^{\circ} \mathrm{C}$ any longer, suggesting a role of Rh1 in warm sensation (Shen et al., 2011). In addition, Rh1 was expressed in body wall neurons also expressing the heat sensing channel dTRPA1, indicating an interaction between Rh1 and dTRPA1 (Shen et al., 2011). Furthermore, norpA mutants lacking PLC were also impaired in warm temperature sensation, suggesting that Rh1 initiates a thermosensory cascade that leads to the opening of dTRPA1 (Kwon et al., 2008; Shen et al., 2011). Although the role of Rh1 in temperature sensation was independent of light, the chromophore retinal was still necessary for warm sensation (Shen et al., 2011).

An additional role of Rh5 and Rh6 in temperature sensation was found. A switch in the temperature preference of larvae was reported, with first, second and early third instar larvae preferring $24^{\circ} \mathrm{C}$, but late third instar larvae preferring $18^{\circ} \mathrm{C}$ (Sokabe et al., 2016). These opsins were found to be coexpressed with ITRPA1 in neurons of the brain and the body wall, and as found for Rh1 (Shen et al., 2011), required PLC and retinal (Sokabe et al., 2016). How opsins sense temperature, however, is still not clear.

In addition to their role in photosensation and thermosensation, opsins seem to have a role in mechanosensation. Two opsins, Rh5 and Rh6, were found to be indispensable for hearing in adult flies (Senthilan et al., 2012). The main auditory organ of the fly, the Johnston's organ, is found in the second antennal segment and represents a chordotonal organ (Nadrowski et al., 2011). Lack of Rh5 and Rh6 lead to a loss of mechanical amplification of sound and a reduction in the sound evoked potentials (Senthilan et al., 2012). However, whether these opsins act as mechanosensors or the mechanisms they are mediating in mechanosensory neurons is not clear. 


\subsubsection{Larval chordotonal organs and mechanosensory channels}

The body wall of larvae has two single ventral chordotonal organs (chos), one single lateral cho, and a group of five (pentameric cho, Ich5). In addition to touch and vibrations (Caldwell et al., 2003; Zhang et al., 2013), chos in larvae are essential for proprioception (Caldwell et al., 2003). Locomotion of larvae consists of peristaltic contractions, where contraction and elongation of each of the muscles in each larval segment generates a wave that propagates from the posterior to the anterior end of the animal (Hughes and Thomas, 2007; Wang et al., 1997). Central pattern generators are in charge of these rhythmic motor patterns, which may be altered or modulated by sensory feedback (Caldwell et al., 2003; Dickinson, 2000; Hughes and Thomas, 2007; Pearson, 1995). Larvae lacking chos or with severe cho mutations (and thus lacking proprioceptive feedback during locomotion) have reduced speeds, persistence, and body amplitude contractions, and have an increased turning behaviour (Caldwell et al., 2003).

Chos of Drosophila adults and larvae express one TRPN and two TRPV mechanosensory channels that are essential for the transduction of mechanical stimuli: NOMPC, Inactive and Nanchung (Cheng et al., 2010; Gong et al., 2004; Kim et al., 2003; Walker et al., 2000; Zhang et al., 2013). NOMPC belongs to the TRPN subfamily and is expressed in mechanosensory and thermosensory neurons (Cheng et al., 2010; Turner et al., 2016; Walker et al., 2000; Yan et al., 2013). NOMPC can be activated by mechanical stimuli, and its expression in non-mechanosensory neurons makes them mechanosensory (Yan et al., 2013). In its N-terminus, NOMPC has 29 ankyrin repeats (Figure 21B) (Walker et al., 2000), which form a helix that is associated with microtubules, and it is believed to be the gating spring of the channel (Howard and Bechstedt, 2004; Yan et al., 2013). In chos, NOMPC is expressed in the distal tip of the cilium (Figure 21C) (Cheng et al., 2010), and is necessary for audition, vibration sensing, and locomotion (Cheng et al., 2010; Effertz et al., 2011; Walker et al., 2000; Zhang et al., 2013).

Nanchung and Inactive are members of the TPRV subfamily and also mediate mechanical transduction in chos (Gong et al., 2004; Kim et al., 2003; Lehnert et al., 2013; Zhang et al., 2013). Both channels are localised in the proximal region of the cilium (Figure 21C) and absence of one channel leads to the absence of the other (Gong et al., 2004; Kim et al., 2003). In this proximal ciliary region, both channels form a calcium conducting heteromultimeric channel (Figure 21A) (Gong et al., 2004; Nesterov et al., 2015). These heteromultimeric channels, however, cannot be activated mechanically, and how they contribute to mechanotransduction is still not clear (Göpfert and Henning, 2016; Nesterov et al., 2015). In has been shown that a GPCR - Latrophilin/ dCIRL - is expressed in larval chos and modulates ionotropic receptor currents and increases the sensitivity 
of the neurons via a cAMP signalling cascade (Scholz et al., 2015, 2017). Although Latrophilin/ dCIRL localised in the same region as NOMPC (Scholz et al., 2017), whether the pathway interacts with NOMPC or the Nanchung/Inactive heteromultimeric channel remains to be analysed.
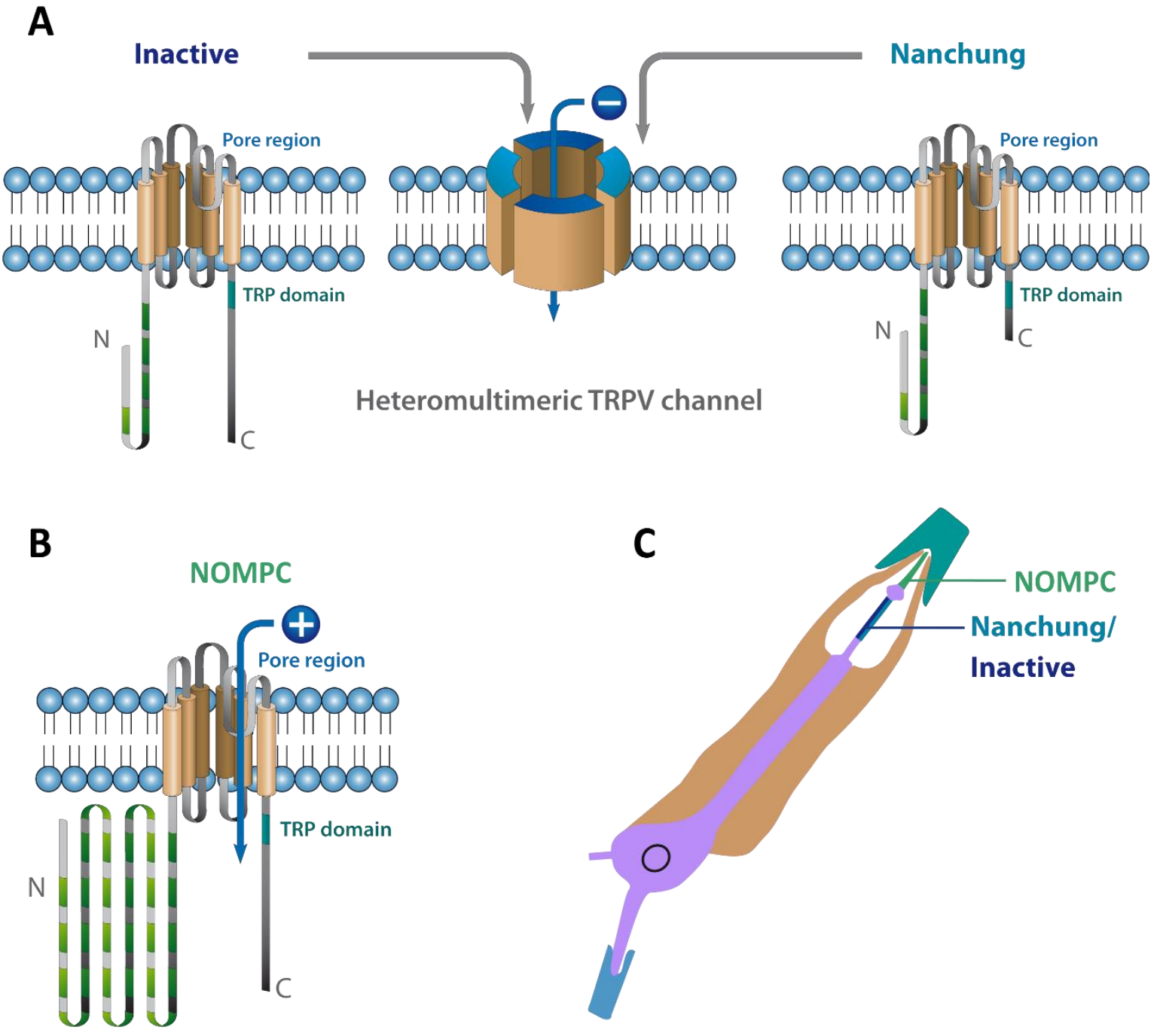

Figure 21. Mechanosensory channels of chordotonal organs. A) Inactive and Nanchung are TRPV channels that form a heteromultimeric channel in chordotonal organs. B) NOMPC C is a TRPN channel expressed in chordotonal organs. The 29 ankyrin repeats in the N-terminus (shown in green) are believed to form the gating spring necessary for mechanical activation of the channel. C) Localisation of NOMPC, Nanchung, and Inactive. NOMPC localises in the distal tip of the cilium, and Nanchung and Inactive in the more proximal part where they form the heteromultimeric channel. Modified from Dr. R. H. Geurten 


\subsubsection{Chordotonal organs and opsins}

It has been shown that Rh5 and Rh6 are required for hearing in adult Drosophila suggesting a role of opsins in chos (Senthilan et al., 2012). Recently, expression of Rh1 and Rh6 in the larval pentameric chordotonal organ (Ich5) in the body wall was found by Dr. Zanini (Figure 22). Unlike the mechanosensory channels (Figure 21C), the localisation is restricted to the dendritic inner segment, below the cilium (Figure 22). As mentioned above, chos are required for proper locomotion in larvae since they provide sensory feedback (Caldwell et al., 2003). However, it has been reported that they also sense warm temperatures (Liu et al., 2003). Since opsins have been implicated in mechanosensation and thermosensation (Senthilan et al., 2012; Shen et al., 2011; Sokabe et al., 2016), it is possible that Rh1 and Rh6 may be serving a role in one of these modalities in larval Ich5.

A
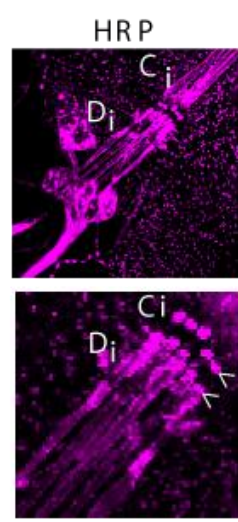

$\mathrm{Rh} 1$
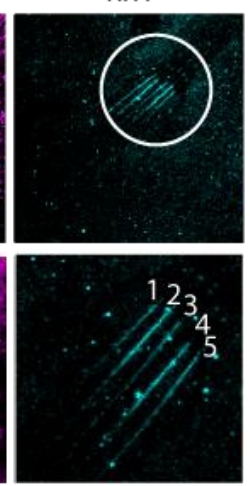

Merge
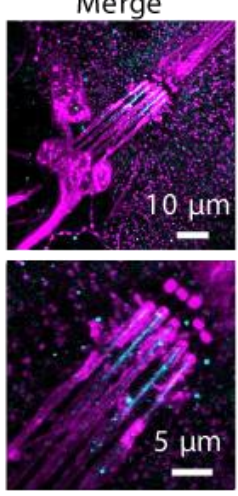

B
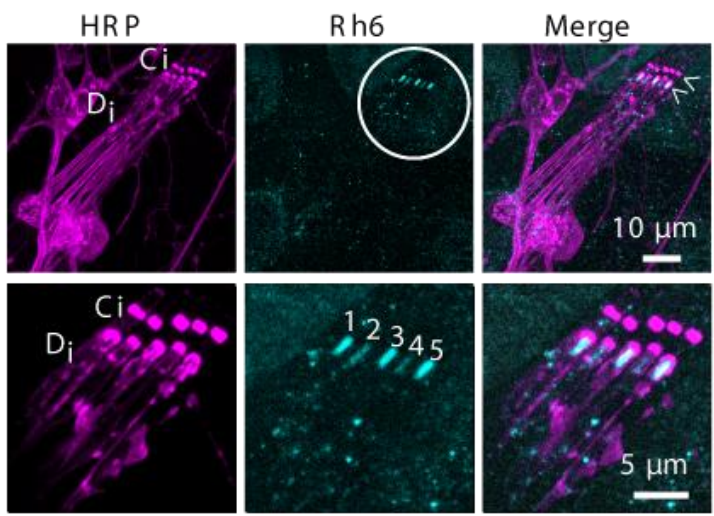

Figure 22. Rh1 and Rh6 localisation in Ich5. A) Anti-NINAE staining showing expression of ninaE in the Ich5. The panels on the left (top and bottom) show the staining of the Ich5 neurons with anti-HRP. The top panel in the middle shows expression of ninaE in the five neurons of the Ich5. The bottom panel is an amplification of the circled region in the top panel and the numbers indicate each of the five neurons. The panels in the right show a merge of both stainings, indicating that the localisation is restricted to the dendritic inner segment $\left(D_{i}\right)$ and not the cilium $\left(C_{i}\right)$. B) Same as $A$, but showing expression of $R h 6$ using anti-Rh6. The localisation is also restricted to the $\mathrm{D}_{\mathrm{i}}$, but in a smaller region than ninaE. Modified from Zanini, Giraldo, et al., 2018.

\subsubsection{Chapter overview}

This chapter encompasses the role of Rh1 and Rh6 in larval chos. To characterise their role, I carried out behavioural experiments of different opsin mutants to determine their role in locomotion, and did cho specific knockdowns to test if such function is due to opsin requirement in chos. I also carried out physiological experiments using calcium imaging to see if cho responses were affected by lack of opsins. Finally, I checked the anatomy of the chos to determine if the structure of the neurons was opsin dependent. The results of this chapter were published as Zanini, Giraldo, et al. 2018 in the journal Neuron. 


\subsection{Results}

\subsubsection{Rhodopsins are required for locomotion}

Larval locomotion of $3^{\text {rd }}$ instar larvae was studied using the assay described in sections II.III.I. and II.VI. The crawling speed of ninaE ${ }^{17}$ mutants, which lack Rh1, was significantly reduced when compared to CantonS and $w^{1118}$ controls (Figure 23A, B). In addition, these larvae turned more often, took more time to advance one body length and covered less distance between consecutive turns (Figure 23B). The phenotype observed is independent of light since the larvae were tested under infrared light that cannot be detected by Drosophila opsins. Similar locomotion defects were observed nina $E^{17}$ mutants crossed with the deficiency $D f(3 R) B S C 636$ ( $n i n a E^{17} / d f$, Figure 23B), which confirmed that the effects observed were in fact by the lack of Rh1, and not by other mutations that may be present in the mutant lines tested. Even though $w^{1118}$ larvae showed a reduced locomotor performance in the crawling phases (i.e., speed and time/ body length), the mutant and the deficiency performed much worse (Figure 23B). Normal locomotion was restored when the genomic mutation was rescued with a ninaE rescue construct, $P\left\{n i n a E^{+}\right\}$, inserted into the $\operatorname{nina}^{17}$ mutant background (Figure 23A, B).

$R h 6^{1}$ larvae also showed a defect in locomotion. Similar to nina $E^{17}$ mutants, $R h 6^{1}$ and $R h 6^{1} / d f$ larvae had decreased speed of locomotion and inter-turn distance, and an increase in the frequency of turning and time to advance one body length (Figure $23 \mathrm{C}$ ). As in nina ${ }^{17}$ mutants, the phenotype observed was independent of light. Normal locomotion was restored with the introduction of an $R h 6$ genomic rescue construct, $P\left\{R h \sigma^{+}\right\}$, into the $R h 6^{1}$ mutant background (Figure 23C). nina $E^{17}, R h 6^{1}$ double mutants had a crawling deficit in all crawling parameters tested when compared to wild-type (Figure 24A). The deficit was similar to that of $n i n a E^{17}$ and $R h 6^{1}$ mutants (Figure 24A). This indicates that Rh1 and Rh6 operate in the same pathway, and they are both essential for locomotion.

Locomotion was also studied in $n a n^{36 a}$ and $i a v^{1}$ larvae, which lack mechanosensory ion channels Nanchung and Inactive necessary for cho function (Zhang et al., 2013). These larvae showed similar deficits like ninaE $E^{17}$ and $R h 6^{1}$ mutants, with decreased speed and inter-turn distance, and an increase in the number of turns and time to advance one body length (Figure 24B). 
A

Cantons
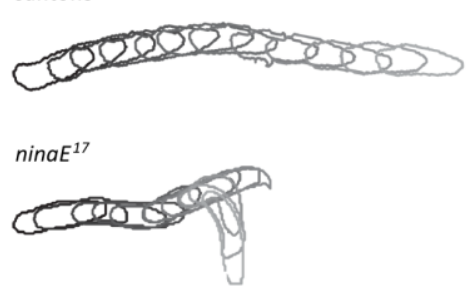

ninaE $E^{17} / D f$

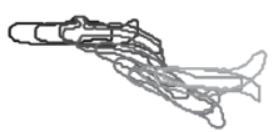

ninaE $E^{17}, P\left\{\right.$ ninaE $\left.^{+}\right\}$
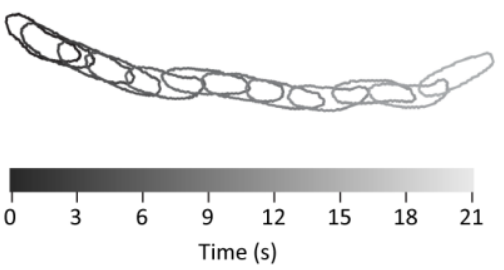

Figure 23. Locomotion of opsin mutants. A) Example trajectories of wild-type (CantonS), ninaE ${ }^{17}$ and nina $E^{17} / D f(3 R) B S C 636 \quad\left(=\right.$ nina $\left.^{17} / D f\right)$ mutants, and nina $E^{17}, P\left\{\right.$ ninaE $\left.E^{+}\right\}$rescue larvae during 21 seconds. B) Quantification of locomotion behaviour of $n i n a E^{17}$ and $n i n a E^{17} / D f$ mutants compared to two wild-type strains (Cantons and $w^{1118}$ ) and nina $E^{17}, P\left\{n i n a E^{+}\right\}$rescue larvae. C) Quantification of locomotion behaviour of $R h 6^{1}$ and $R h 6^{1} / D f$ compared to two wild-type strains and $R h 6^{1}, P\left\{R h \sigma^{+}\right\}$ rescue larvae. The black line indicates the median, the box marks the lower and upper quartile, and the whiskers the range of the entire data set in the case where there are no outliers, and in the case outliers are present the 1.5 interquartile distance; and the outliers are indicated by black crosses. Box colours: grey $=$ wild-type, green $=$ mutants and purple $=$ rescue strains. Significant differences from wild-type (Fisher's exact permutation tests with Benjamini - Hochberg correction): ${ }^{*}=p$ $<0.05,{ }^{* *}=\mathrm{p}<0.01, * * *=\mathrm{p}<0.001$, NS $=$ not significant. $\mathrm{n}$ : CantonS $=21, w^{1118}$ $=30, \operatorname{nina} E^{17}=30, \operatorname{nina} E^{17} / d f=30$, nina $E^{17}, P\left\{\right.$ nina $\left.E^{+}\right\}=30, R h \sigma^{1}=30$, $R h 6^{1} / d f=29, R h 6^{1}, P\left\{R h 6^{+}\right\}=21$.
B
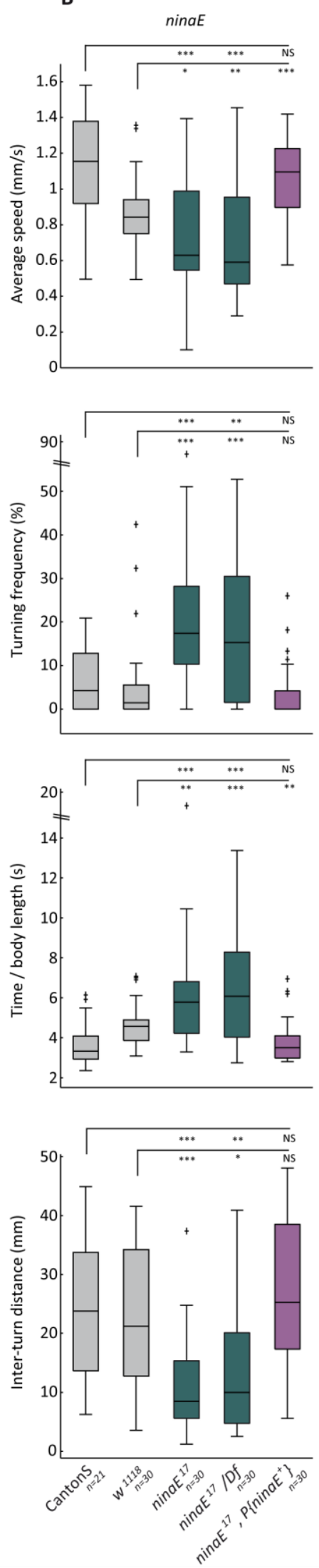

C
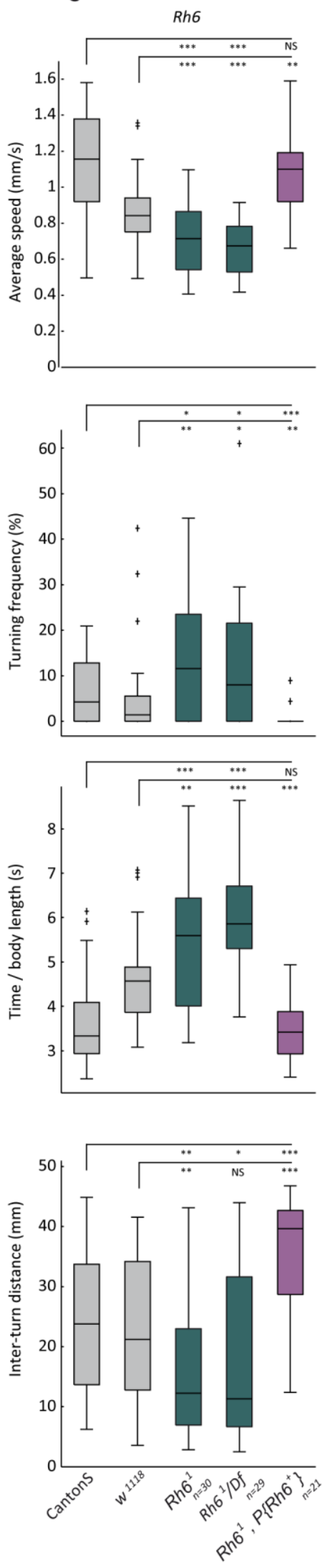
A
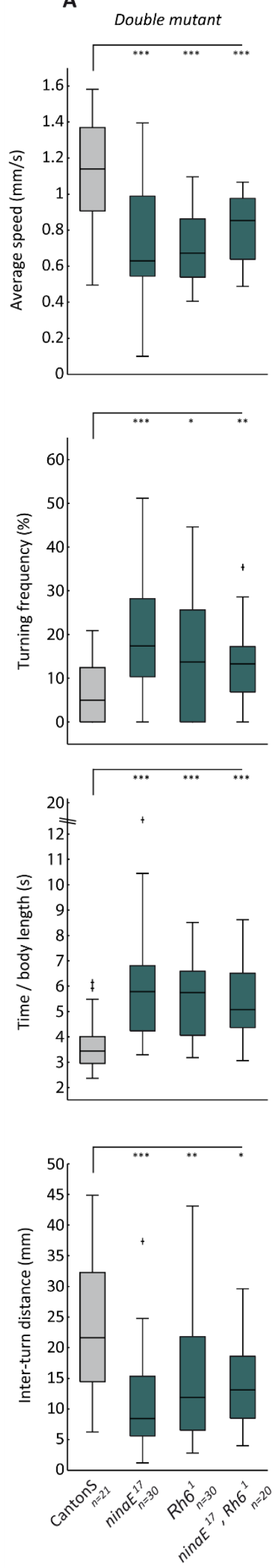

B
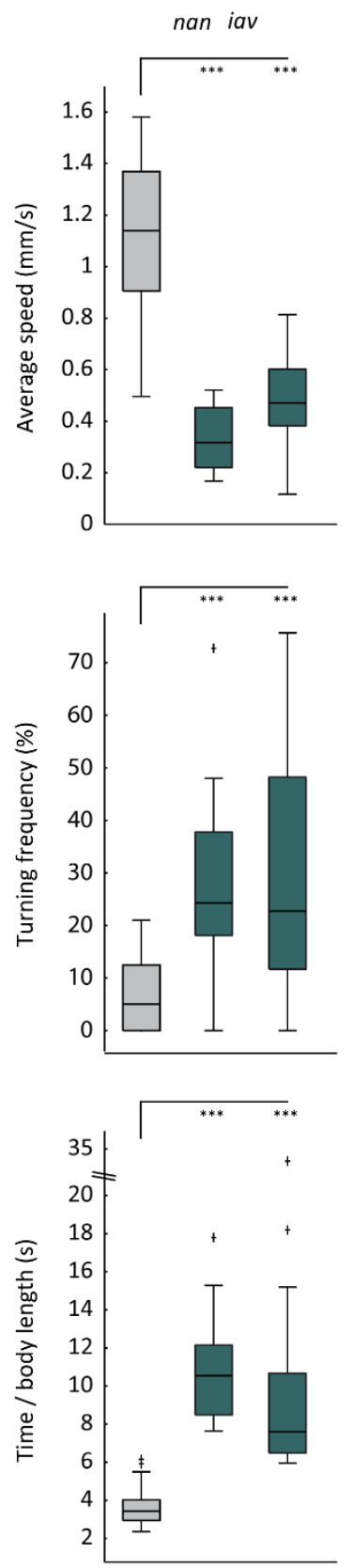

Figure 24. Locomotion of mechanosensory ion channel mutants and nina $E^{17}$ - $\boldsymbol{R h}^{\mathbf{1}}$ double mutants. A) Quantification of locomotion behaviour in $n a n^{36 a}$ and $i a v^{1}$ mutants with silenced cho receptors. B) Quantification of locomotion behaviour in nina $E^{17}-R h 6^{1}$ double mutants compared to wild-type and single mutants. The data is represented as in Figure 23. Box colours: grey = wild-type and green $=$ mutants. Significant differences from wild-type (Fisher's exact permutation tests with Benjamini - Hochberg correction): ${ }^{*}=p<0.05, * *=p<$ $0.01, * * *=p<0.001 . n$ : Cantons $=21, n i n a E^{17}=30, R h 6^{1}=30$, nina $E^{17}, \operatorname{Rh}^{1}=20, \operatorname{nan}^{36 a}=16$, $i a v^{1}=17$. 
Larval crawling happens by contraction of the body wall muscles, and sensory feedback from the muscles is required for proper contraction. Larval peristalsis was studied using the assay described in section II.III.II.II. The contraction amplitude of $n i n a E^{17}$ and $R h 6^{1}$ mutants during forward crawling was significantly reduced (Figure $25 \mathrm{~A}$ ). However, the contraction defects were ameliorated by the ninaE $E^{17}, P\left\{n i n a E^{+}\right\}$rescue, and fully rescued in the $R h \sigma^{1}, P\left\{R h \sigma^{+}\right\}$larvae (Figure 25A). The reduction in contraction amplitude was also observed when the individual segment contraction of both mutants was measured by calculating the $\Delta F / F$ of muscles expressing GFP (see section II.III.II.I.) (Figure 25B). In addition, the reduction in the contraction amplitude was also observed in $n a n^{36 a}$ and $i a v^{1}$ mutants (Figure 25A).

A

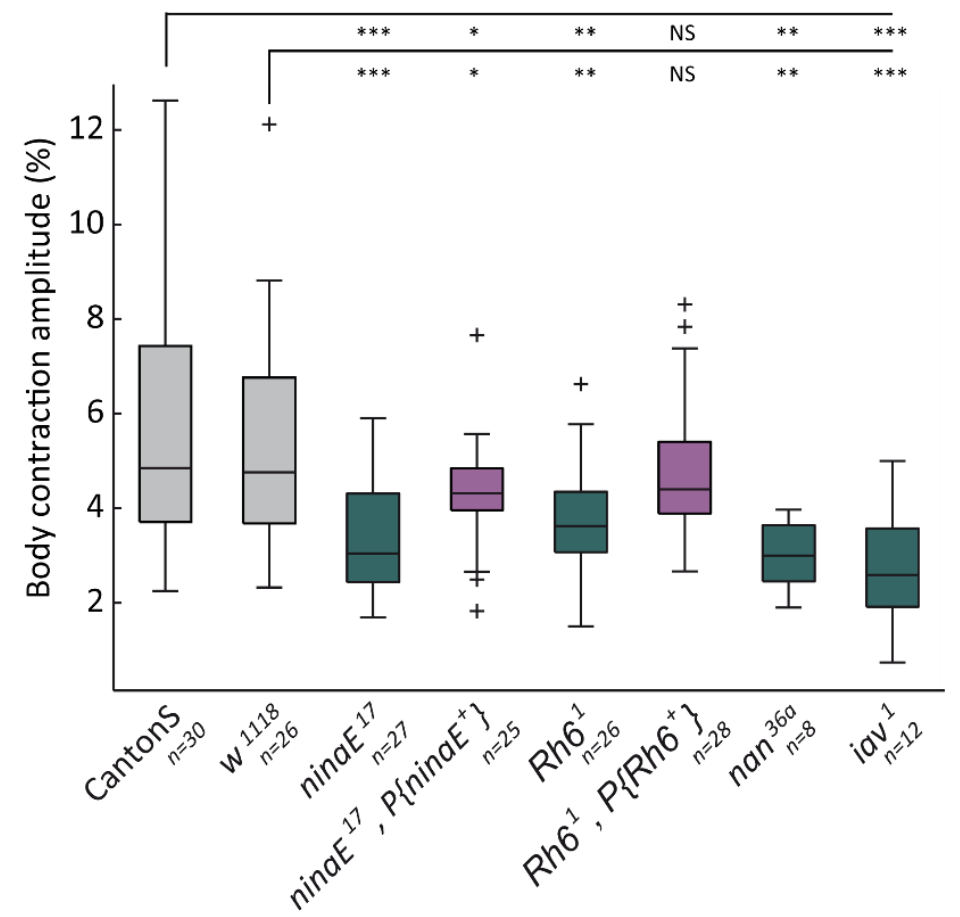

B

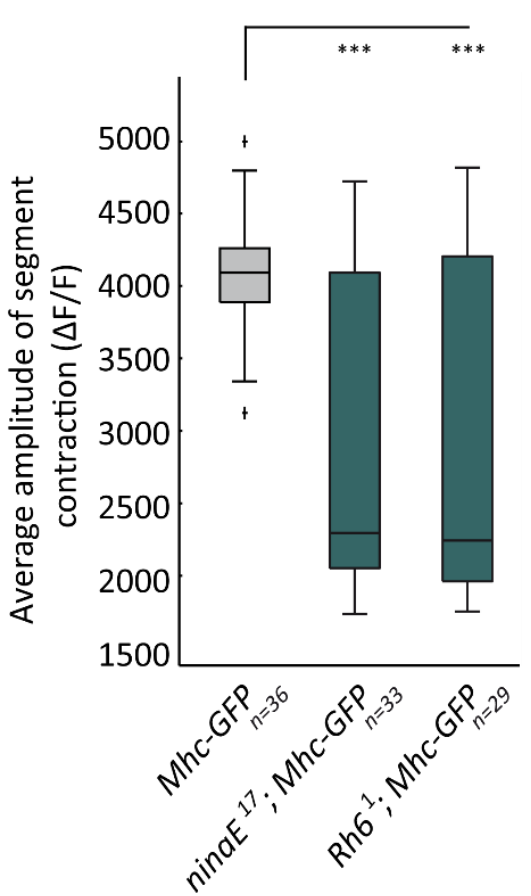

Figure 25. Peristaltic contraction quantification. A) Relative amplitude of locomotor body contractions normalised to individual body lengths. B) Average amplitude of segment contraction of larvae expressing GFP in the muscles. The amplitude of contraction is quantified as the $\Delta F / F$ seen in each segment as they contract. Box colours: grey = wild-type, green $=$ mutants, purple $=$ genomic rescues. The data is represented as in Figure 23. Significant differences from wildtype (Fisher's exact permutation tests with Benjamini - Hochberg correction): * $=p<0.05, * *=p<0.01, * * *=p<0.001$, $\mathrm{NS}=$ not significant. $\mathrm{n}$ : CantonS $=30, w^{1118}=26, \operatorname{ninaE}^{17}=27, \operatorname{ninaE}^{17}, P\left\{\right.$ ninaE $\left.^{+}\right\}=25, R h 6^{1}=26, R h 6^{1}, P\left\{R^{\prime} 6^{+}\right\}=28, . n a n^{36 a}$ $=8, \operatorname{iav}^{1}=12$, Mhc-GFP $=36, n i n a E^{17}, M h c-G F P=33, R h 6^{1}, M h c-G F P=29$.

\subsubsection{Chordotonal organs need Rhodopsin 1 for proper function}

The deficits in locomotion observed for ninaE $E^{17}$ and $R h 6^{1}$ suggest that these opsins may play a role in proprioception. In addition, the similarity of the phenotypes observed to nan $^{36 a}$ and $i a v^{1}$ mutants indicates that such function may be in chos. To prove this, first, the role of chos was confirmed. Cho neurons were ablated by expressing the apoptotic factors Hid and Reaper (Grether et al., 1995; 
White et al., 1994) in cho neurons only. This was done by crossing a line carrying the cho specific driver Dhc93Ab-Gal4 (Senthilan et al., 2012) with UAS-hid,rpr line. Even though the Dhc93Ab-Gal4 line showed a slight impairment compared to the UAS-hid,rpr line, the resulting cross had a much stronger phenotype than both parental lines. As observed for opsin and mechanosensory ion channel mutants (Figure 24), larvae lacking cho neurons had a significant decrease in the speed and inter-turn distance, and a significant increase in the frequency of turning and time to advance one body length (Figure 26A). These results confirm the role of chos in larval crawling, and that lack of cho function leads to similar crawling deficits like the ones found for ninaE $E^{17}$ and $R h 6^{1}$ mutants.

ninaE was knocked down in chos by expressing interference RNA (RNAi) for ninaE in cho neurons using the cho specific dnai2-Gal4 (Karak et al., 2015). An impairment in crawling similar to that of opsin mutants, mechanosensory ion channel mutants, and cho ablated larvae was observed (Figure 26B). This confirmed the role of ninaE in chos for proprioception, and that the phenotype observed in nina $E^{17}$ mutants most likely comes from the lack of Rh1 in chos.

The responses of the lateral pentameric cho (Ich5) to mechanical stimuli were studied using calcium imaging. Larval Ich5 neurons were stimulated with $20 \mathrm{~Hz}$ and amplitudes ranging from $0.5 \mu \mathrm{m}$ to $5 \mu \mathrm{m}$, and the increase in the fluorescent GCaMP6f signal to these stimuli was measured. Wild-type responses increased with higher amplitude of stimulation when the whole organ or only the dendrites were analysed (Figure 27A, B). This response was non-linear since a higher increase in the response to increasing amplitude of stimulation was observed at lower amplitudes than at higher amplitudes. nina $E^{17}$ mutants had significantly lower amplitude responses compared to wildtype, and the increase in the response with higher amplitude was not as evident (Figure 27A, B). The responses of the mutants lost their non-linearity, which suggests that lack of Rh1 results in a reduced sensitivity of the organs. The mutant phenotype was partially ameliorated with the genomic rescue $n i n a E^{17}, P\left\{n i n a E^{+}\right\}$, especially in the dendrite response (Figure 27A, B). The whole organ response was more variable since the cell bodies were more likely to move out of focus during a recording than the dendrites. Only those organs that showed a response above $1 \%$ to $4 \mu \mathrm{m}$ or $5 \mu \mathrm{m}$ were taken into account for the analysis. Interestingly, $66.6 \%$ of the wild-type organs (total of 24 organs measured) showed dendrite responses, whereas in the nina $E^{17}$ mutants (total of 20 organs measured) and the $n i n a E^{17}, P\left\{n i n a E^{+}\right\}$rescue (total of 22 organs measured) only $45 \%$ showed a response. It is not clear whether this difference is due to the mutation or problems during the experimental procedure. 
A
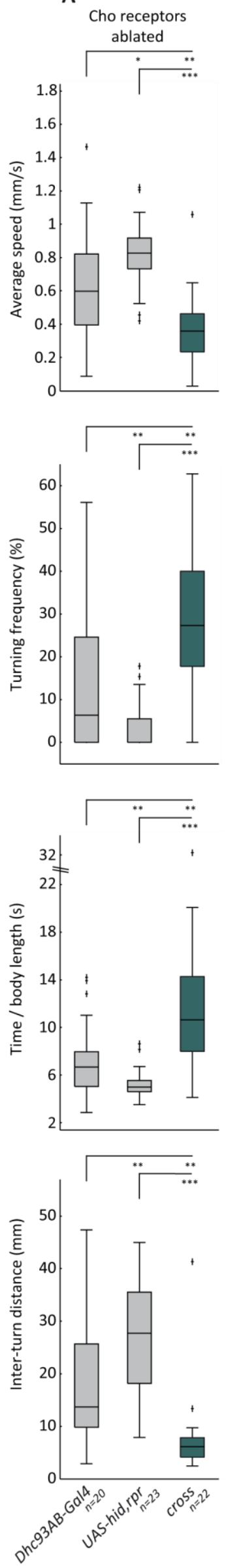

ninaE knocked down in chos
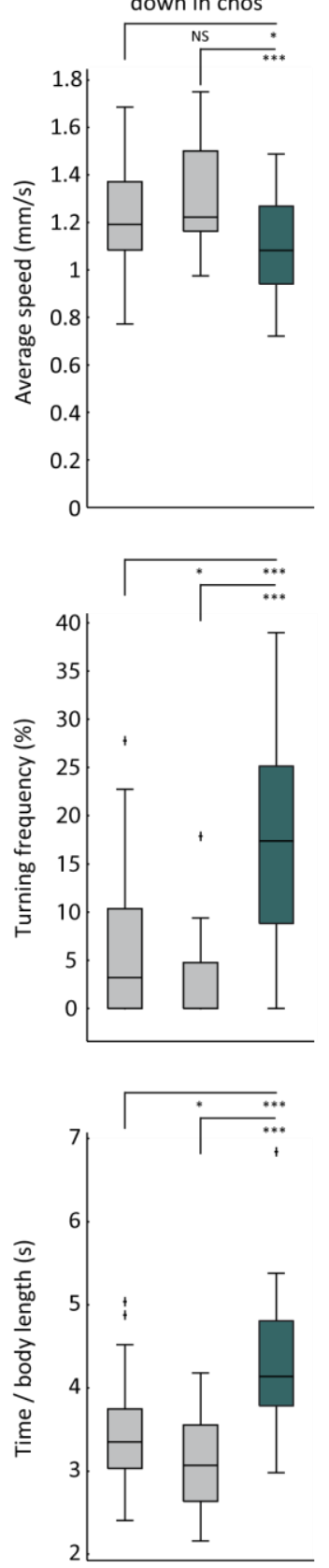

Figure 26. Locomotion of chordotonal receptorablated larvae and ninaE chordotonal knockdown larvae. A) Quantification of locomotion of larvae with ablated cho neurons by driving the expression of the apoptotic factors Hid and Reaper with the cho specific driver DhC93ABGal4. . n: DhC93AB-Gal4 = 20, UAS-hid, $r p r=23$, cross $=22$. B) Quantification of locomotion behaviour of larvae with a knock down of ninaE in cho neurons by driving RNAi for ninaE in chos using the cho specific driver dnai2-Gal4. $\mathrm{n}$ : dnai2-Gal4 = 30, UAS-ninaE$R N A i=30$, cross $=33$ The data is represented as in Figure 23. Box colours: grey $=\mathrm{Gal} 4$ and UAS controls and green $=$ cross. Significant differences from parental controls (Fisher's exact permutation tests with Benjamini - Hochberg correction): $*=\mathrm{p}<0.05, * *=\mathrm{p}$ $<0.01, * * *=p<0.001$, NS $=$ not significant. 
A

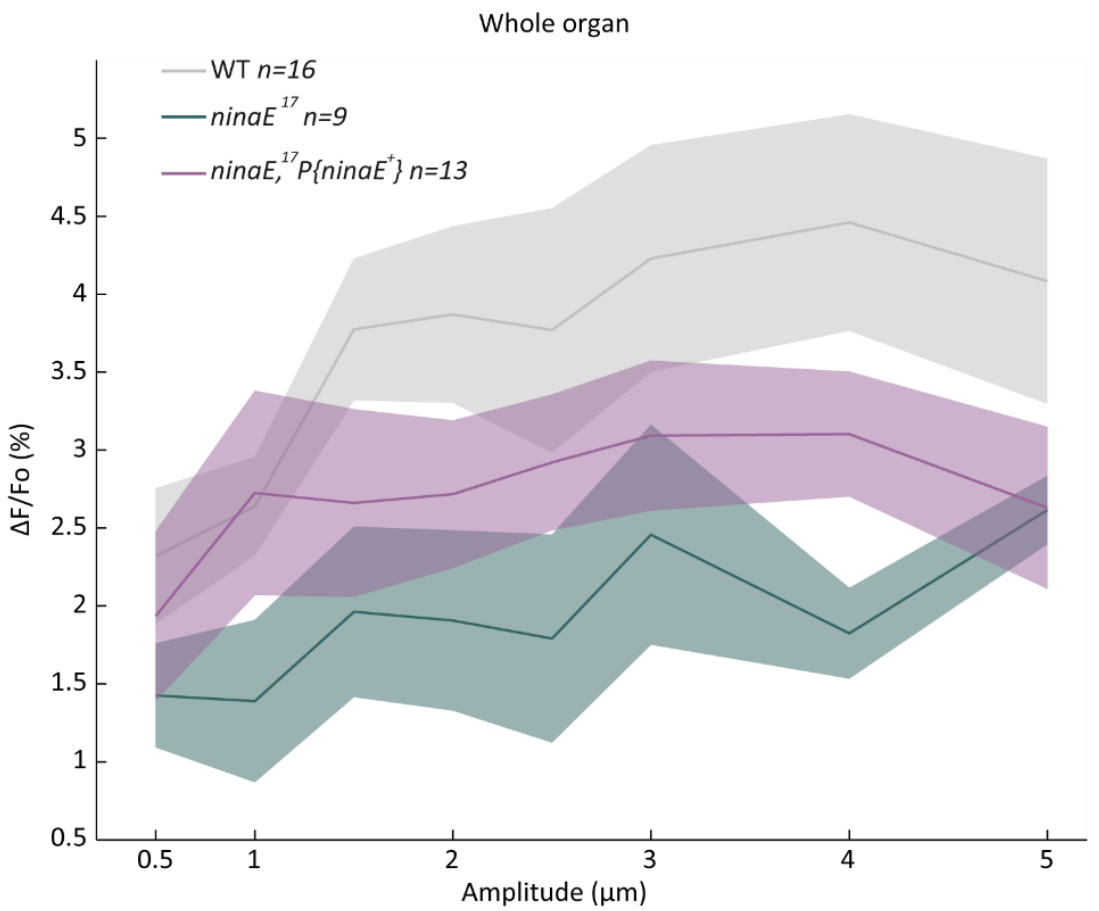

\begin{tabular}{|l|c|c|c|c|c|c|c|c|}
\cline { 2 - 9 } \multicolumn{1}{c|}{} & \multicolumn{9}{c|}{ Amplitude $(\mu \mathrm{m})$} \\
\cline { 2 - 9 } \multicolumn{1}{c|}{} & 0.5 & 1 & 1.5 & 2 & 2.5 & 3 & 4 & 5 \\
\hline ninaE $^{17}$ & $*$ & $* *$ & $* *$ & $* *$ & $*$ & NS & $* *$ & NS \\
\hline ninaE $^{17}, P\left\{\right.$ ninaE $\left.^{+}\right\}$ & NS & NS & $* *$ & $*$ & $*$ & NS & $*$ & $*$ \\
\hline
\end{tabular}

\section{B}

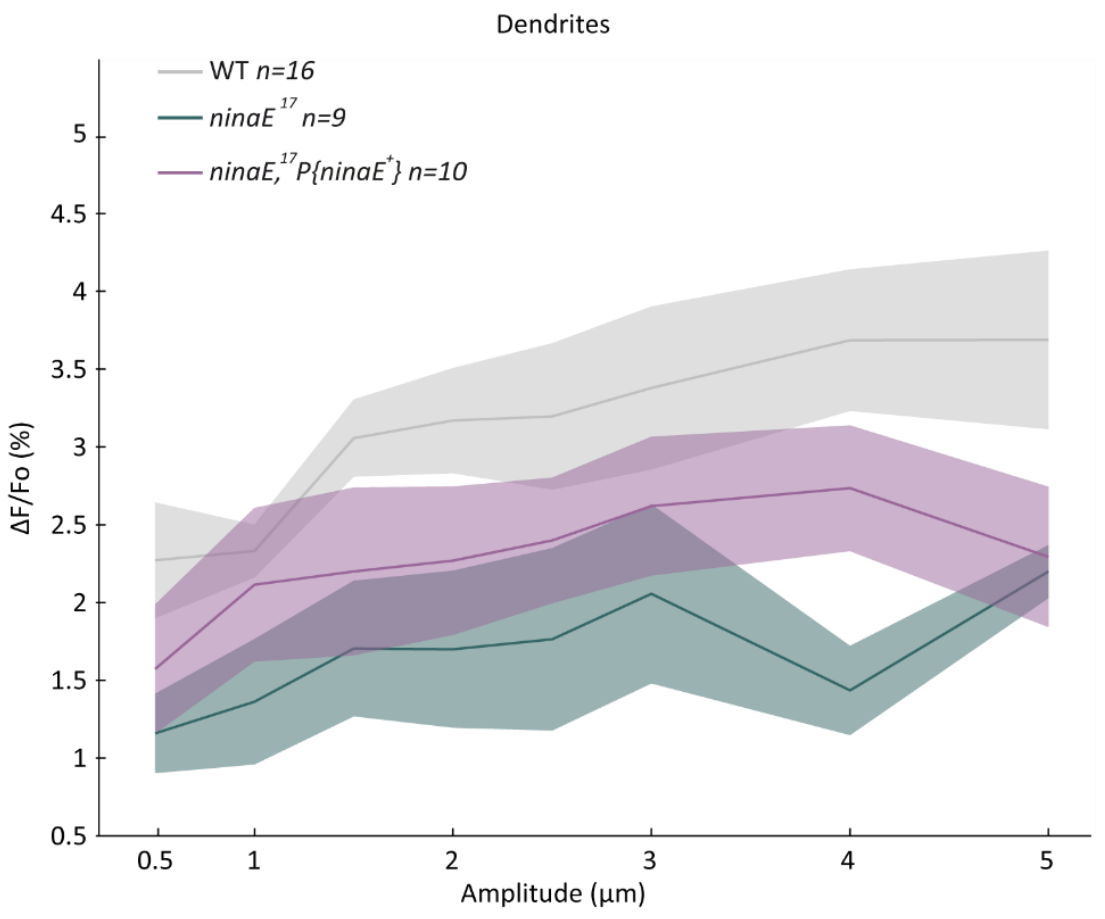

\begin{tabular}{|l|c|c|c|c|c|c|c|c|}
\cline { 2 - 9 } \multicolumn{1}{c|}{} & \multicolumn{9}{c|}{ Amplitude $(\mu \mathrm{m})$} \\
\cline { 2 - 9 } \multicolumn{1}{c|}{} & 0.5 & 1 & 1.5 & 2 & 2.5 & 3 & 4 & 5 \\
\hline nina $^{17}$ & $* *$ & $* *$ & $* *$ & $* *$ & $* *$ & $*$ & $* * *$ & $* *$ \\
\hline nina $^{17},{\text { P }\left\{\text { ninaE }^{+}\right\}}$ & NS & NS & NS & NS & NS & NS & NS & $*$ \\
\hline
\end{tabular}

Figure 27. Mechanically evoked calcium responses of Ich5 neurons. Responses of $A$ ) the whole organ and B) the dendrites only to a sinusoidal mechanical stimulus of $20 \mathrm{~Hz}$ of wild-type, nina ${ }^{17}$ and $\operatorname{nina} E^{17}, P\left\{\right.$ ninaE $\left.^{+}\right\}$rescue expressing the calcium sensor GCaMP6f. The line represents the mean and the error corresponds to the SEM. The table under each plot shows the statistical analysis comparing the wild-type response to the mutants and rescue (Fisher's exact permutation tests): $*=p<$ $0.05, * *=p<0.01, * * *=p<$ 0.001, NS $=$ not significant. A) $n$ : $\mathrm{WT}=16, \operatorname{nina} E^{17}=9, \operatorname{nina} E^{17}$, $P\left\{\right.$ ninaE $\left.^{+}\right\}=13$. B) $\mathrm{n}: \mathrm{WT}=16$, nina $E^{17}=9, \operatorname{nina} E^{17}, P\left\{\right.$ nina $\left.E^{+}\right\}=$ 10 


\subsubsection{Rhodopsin function in chos is not related to thermosensation}

Rh1 and Rh6 have been reported to interact with the heat sensing channel dTRPA1 and to be required for warm temperature sensation (Shen et al., 2011; Sokabe et al., 2016). Neurons expressing dtrpA1 were ablated by driving UAS-hid,rpr with trpA1-gal4. Locomotion of larvae with dtrpA1 ablated neurons was not largely affected (Figure 28A). Although there is a difference in the performance between the parental line UAS-hid,rpr, the differences seen were in the opposite direction as those observed for opsin mutants, meaning these larvae had better locomotion than the parental line. In addition, $d \operatorname{trpA1}$ neuron ablated larvae had similar locomotion to the dtrpA1Gal4 parental line (Figure 28A). The only parameter that was affected by the ablation was the turning frequency (Figure 28A). Since turning behaviour is closely related to temperature seeking behaviour (Garrity et al., 2010), it is expected that larvae without heat sensing neurons have an impairment in this behaviour.

To exclude that the phenotypes observed in the crawling of opsin mutants were due to problems in temperature seeking behaviour, nina $E^{17}$ and $R h 6^{1}$ mutants were tested at $29^{\circ} \mathrm{C}$ where thermosensation is no longer dependent on Rhodopsins (Shen et al., 2011; Sokabe et al., 2016). The defects seen at $25^{\circ} \mathrm{C}$ (Figure $23 \mathrm{~B}, \mathrm{C}$ ) were also present at $29^{\circ} \mathrm{C}$ (Figure $28 \mathrm{~B}$ ). Some of the effects were less pronounced, probably because the elevated temperature also modified the crawling behaviour of the wild-type larvae. However, the fact that both mutant strains still had an impairment confirmed that the role of Rh1 and Rh6 in proprioception is independent of their thermosensory roles.

Temperature preferences of larvae with a cho knockdown of ninaE were assessed in a Peltier element-based setup as described in section II.III.IV.II. Larvae were placed in the gradient ranging from $12.5^{\circ} \mathrm{C}-28^{\circ} \mathrm{C}$ for 10 minutes and their final temperature distribution was measured resulting in a single distribution per strain. The final distribution of the larvae was corrected for cold trapping using IGLOO (II.V.IV and Chapter 1). The 95\% confidence interval at each temperature of the gradient was calculated per strain to obtain the final corrected preferences (Figure 29A-C).

The three lines tested (dnai2-Gal4, UAS-ninaE-RNAi, and their cross) all had distributions around $18^{\circ} \mathrm{C}$. However, the UAS-ninaE-RNAi parental line distributed at warmer temperatures where the cross and dnai2-Gal4 lines would not (Figure 29A-C). No strong warm avoidance was observed but larvae would not distribute at temperatures higher than $22^{\circ} \mathrm{C}$. The corrected mean preference at the preferred distribution was calculated. Although the larvae with ninaE knocked down in chos preferred significantly lower temperatures than the UAS-ninaE-RNAi parental larvae, 
no difference was observed with the dnai2-Gal4 line (Figure 29D), suggesting that knocking down ninaE does not affect temperature behaviour. In addition, the effect observed against the UASninaE-RNAi line resulted in a reduced temperature preference. Since Rh1 mediates warm temperature sensation, it can be concluded that the thermosensitive role of Rh1 is independent of chos.

A
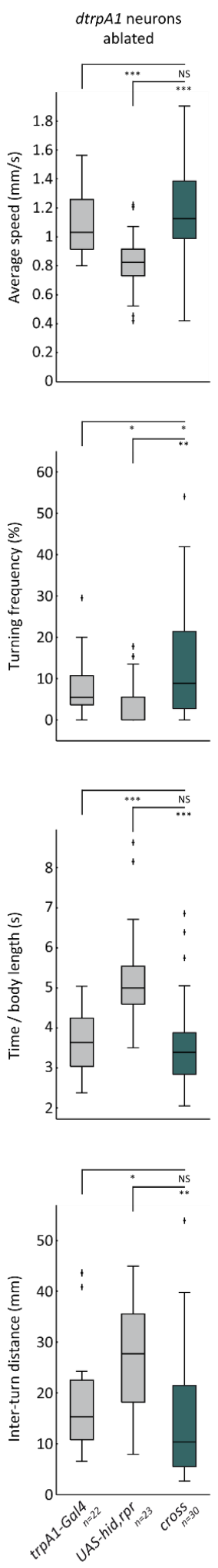

B
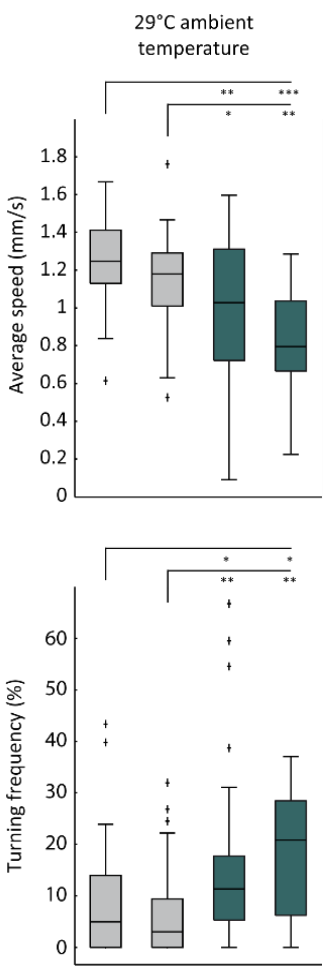

Figure 28. Locomotion of dtrpA1 receptor-ablated larvae and mutant larvae at $29^{\circ} \mathrm{C}$. A) Quantification of locomotion of larvae with ablated dtrpA1 neurons by driving the expression of the apoptotic factors $\mathrm{Hid}$ and Reaper with the trpA1-Gal4. B) Quantification of locomotion behaviour of wild-type and nina $E^{17}$ and $R h 6^{1}$ mutants at $29^{\circ} \mathrm{C}$. The data is represented as in Figure 23. Box colours A: grey $=$ Gal4 and UAS controls and green $=$ cross. Box colours B: grey = wild-type controls and green $=$ mutants. Significant differences from parental controls (A) and wild-type (B) (Fisher's exact permutation tests with Benjamini Hochberg correction): ${ }^{*}=p<$ $0.05, * *=p<0.01, * * *=p<$ $0.001, \mathrm{NS}=$ not significant. $\mathrm{n}$ : trpA1-Gal4 $=22$, UAS-hid, $r p r=$ 23 , cross $=30$, Cantons $=20$, $W^{1118}=28$, nina $E^{17}=43, R h 6^{1}=$ 17. 
A

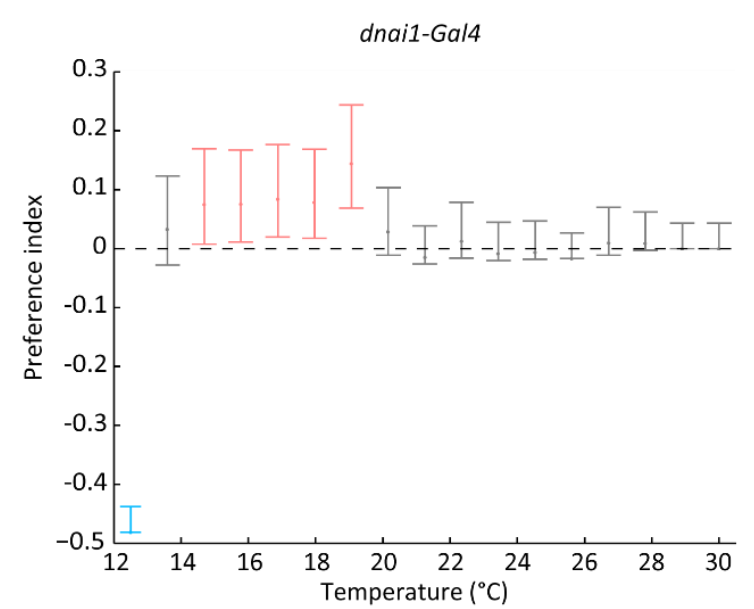

C

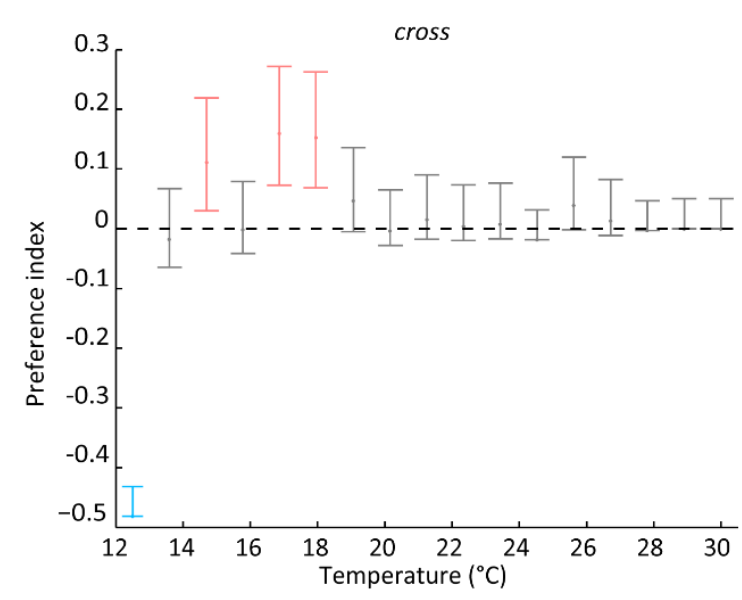

B

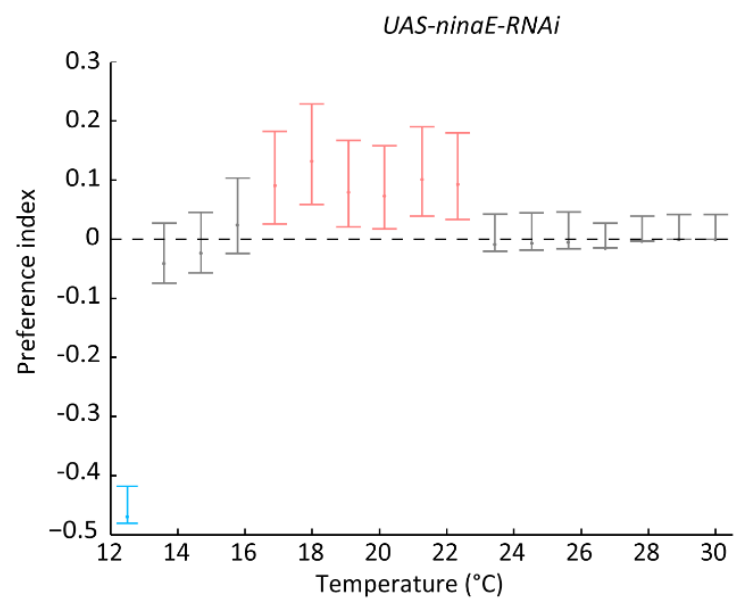

D

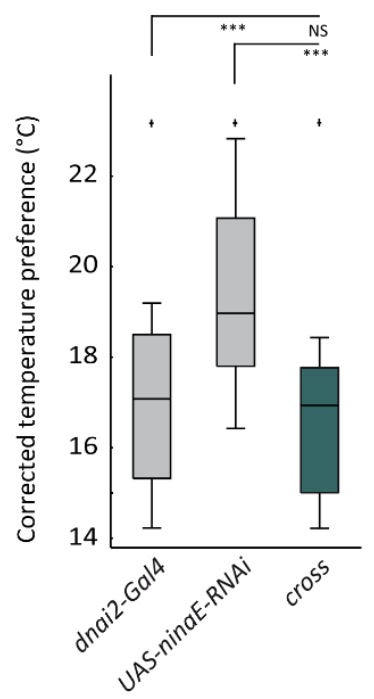

Figure 29. Temperature preferences of larvae with a cho specific ninaE knockdown. A knock down of ninaE in cho neurons by driving RNAi for ninaE in chos using the cho specific driver dnai2-Gal4. A-C) Cold trapped corrected distribution of larvae in a temperature gradient. After the correction (see chapter 1), a preference index is obtained for each temperature. A negative preference index (blue) means the temperature is avoided and a positive index (red) means the temperature is preferred. The points represent the median and the bars represent the $95 \%$ confidence interval. If the $95 \%$ confidence interval overlaps with zero (grey) the temperature is considered as neither preferred nor avoided. A) Gradient distribution of dnai2-Gal4. B) Gradient distribution of UAS-ninaE-RNAi. C) Gradient distribution of the cross of A and B. D) Temperature preferences of the corrected distributions in A-C. Only the temperature values in the preferred temperatures (i.e., red points in $\mathrm{A}-\mathrm{C}$ ) are used to calculate the preference. The data is represented as in Figure 23. Box colours D: grey $=$ Gal4 and UAS controls and green $=$ cross. Significant differences from parental lines (Fisher's exact permutation tests with Benjamini - Hochberg correction): ${ }^{*}=p<0.05,{ }^{* *}=p<0.01, * * *=p<0.001$, NS = not significant. dnai2-Gal4 $\mathrm{n}=83$, UAS-ninaE-RNAi $\mathrm{n}=86$, cross $\mathrm{n}=72$. 


\subsubsection{Locomotion is retinal dependent}

In Drosophila, Rhs bind to a light-sensitive retinal chromophore (3-hydroxy 11-cis-retinal), which is synthesised from carotenoids in the diet (Montell, 2012; Wang et al., 2007). Although the thermosensitive roles of Rhs are independent of light, they still require the chromophore for normal function (Shen et al., 2011; Sokabe et al., 2016). To assess whether the proprioceptive roles of Rh1 and Rh6 are chromophore dependent, the locomotor performance of santa-maria ${ }^{1}$ mutants, which cannot synthesise the chromophore (Wang et al., 2007), was tested. For all the parameters measured, santa-maria ${ }^{1}$ mutants showed a locomotion impairment (Figure 30A), indicating that the proprioceptive functions of Rhodopsins may need the retinal chromophore. The experiments were carried out in complete darkness which excludes that the phenotype observed might be due to a photosensory deficit.

Dietary carotenoids are necessary for chromophore synthesis (Wang et al., 2007). To further confirm the retinal chromophore requirements for locomotion, $w^{1118}$ larvae were grown in carotenoid depleted food for over two generations. These larvae had impaired photosensitivity when tested with the Darth Vader assay (Table 4), which confirmed the lack of chromophore synthesis. Surprisingly, these larvae showed impairment in the crawling phases of locomotion, but no impairment in the turning phases (Figure 30B).

Table 4. Darth Vader assay. A $X^{2} p$-value $<0.05$ indicates that the larvae are not evenly distributed between both sides, suggesting that there is a preference for one of the sides.

\begin{tabular}{|c|c|c|c|}
\hline Strain & Dark & Light & $\mathbf{X}^{2}$ p-value \\
\hline$w^{1118}$ & 17 & 3 & 0.0017 \\
\hline santa-maria $^{1}$ & 9 & 5 & 0.286 \\
\hline$w^{1118}$ vitamin A- & 19 & 21 & 0.669 \\
\hline
\end{tabular}


A

santa-maria
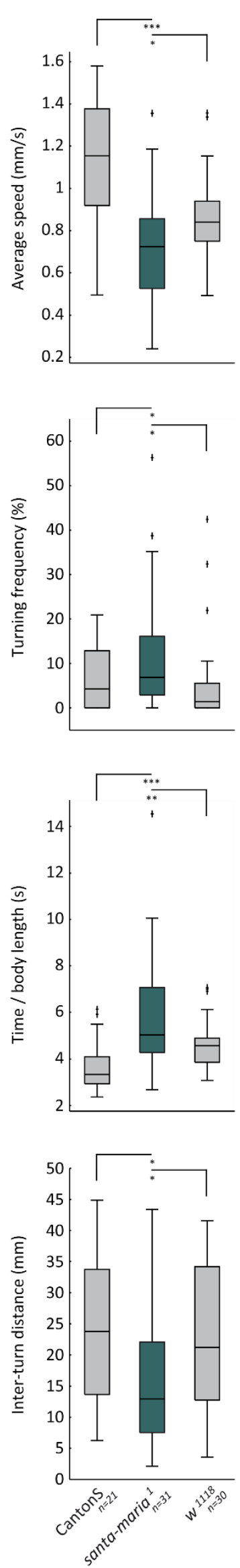

Retinal depleted
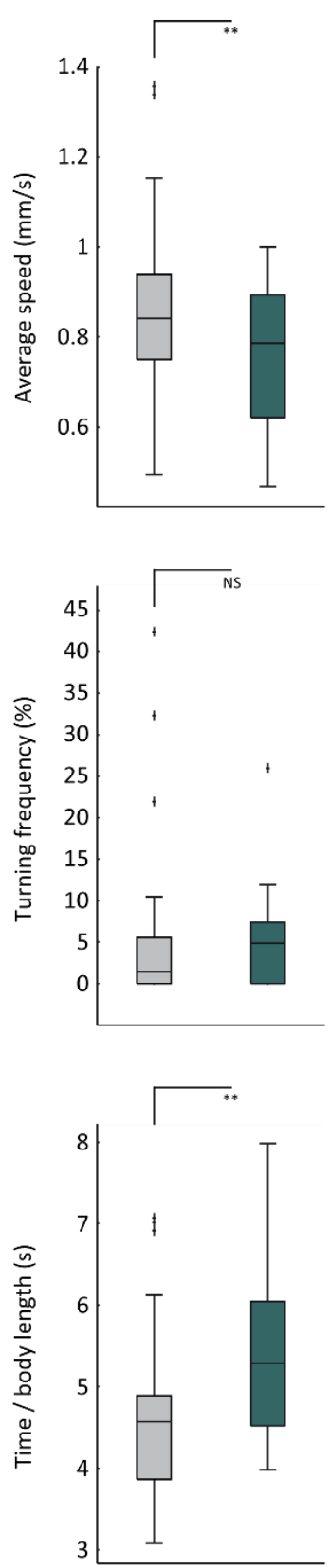

Figure 30. Locomotion of santa-maria mutants and vitamin A depleted larvae. A) Quantification of locomotion santa-maria ${ }^{1}$ mutants that cannot synthesise retinal B) Quantification of locomotion behaviour of $w^{1118}$ larvae grown on vitamin A depleted food. Lack of vitamin A in the food leads to an inability to synthesise retinal. The data is represented as in Figure 23. Box colours: grey $=$ wild-type controls and green = mutants and vitamin A depleted larvae. Significant differences from wild-type (Fisher's exact permutation tests with Benjamini - Hochberg correction): $*=p<0.05, * *=p<$ $0.01, * * *=p<0.001, \mathrm{NS}=$ not significant. $\mathrm{n}$ : CantonS $=21$, santa-maria ${ }^{1}=31, w^{1118}=30$, $w^{1118}$ ret- $=30$. 


\subsubsection{Locomotion requires PLC}

Phospholipase C (PLC) is an intermediary in the phototransduction pathway initiated by Rhodopsins (Bloomquist et al., 1988; Montell, 2012). In addition, it is involved in thermosensation and believed to be part of a thermosensory pathway involving Rh1 and dTRPA1 channels (Kwon et al., 2008; Shen et al., 2011). In Drosophila PLC is encoded by the gene norpA (Bloomquist et al., 1988). To test the role of PLC in proprioception, the locomotor performance of norp $A^{36}$ mutants was assessed. Mutant larvae had a significant decrease in speed, and a significant increase in turning frequency and time to advance one body length (Figure 31A). The inter-turn distance was not affected in these mutants (Figure 31A).

To determine if the role observed for norpA in locomotion is related to chos, expression of norpA was studied in Ich5 neurons by driving GFP using a norpA-Gal4 driver. The GFP signal was enhanced with a chicken anti-GFP primary antibody (concentration 1:1000) and an Alexa Fluor 488 anti-chicken secondary antibody (concentration 1:300). The nervous system was stained with a Cy3conjugated donkey anti-HRP (concentration 1:300). Although a faint GFP signal is observed in the Ich5 neuron cell bodies and dendrites, this was also observed in the UAS-GFP controls, most likely due to some unspecific staining from the anti-GFP antibody. These results suggest that the phenotype observed for norpA $A^{36}$ mutants is independent of Ich5 neurons. 
A
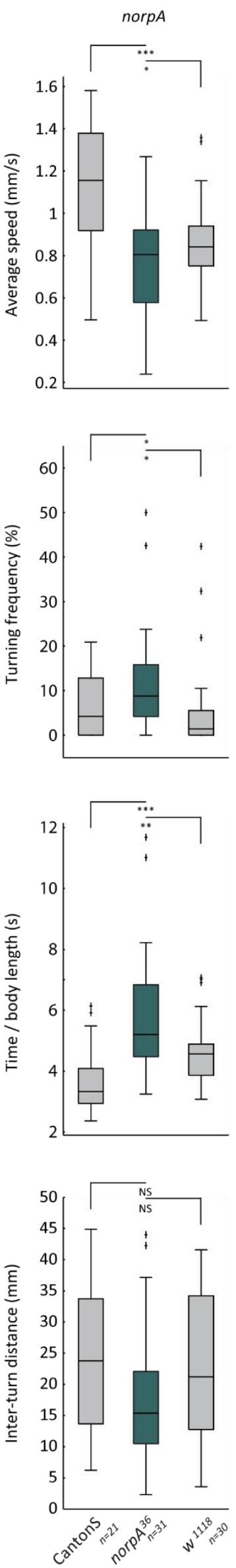

B

norpA-Gal4 x UAS-GFP
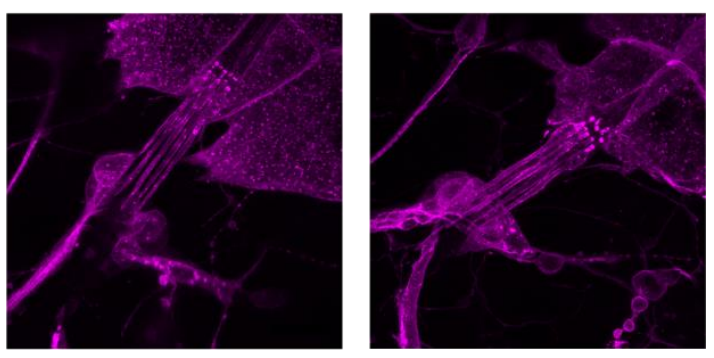

GFP
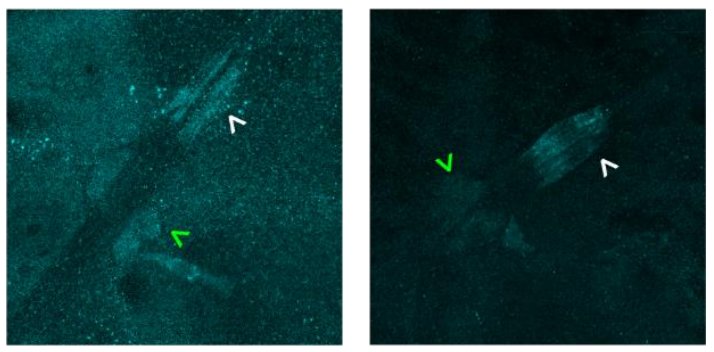

MERGE
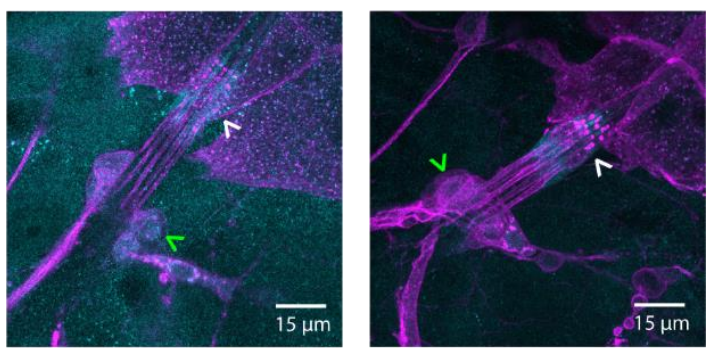

Figure 31. Locomotion of norpA mutants and norpA expression in Ich5 neurons. A) Quantification of locomotion of norp $A^{36}$ mutants that lack PLC. The data is represented as in Figure 23. Box colours: grey $=$ wild-type controls and green $=$ mutants Significant differences from wild-type (Fisher's exact permutation tests with Benjamini - Hochberg correction): ${ }^{*}=p<0.05, * *=p<0.01, * * *=p<0.001$, $\mathrm{NS}=$ not significant. $\mathrm{n}$ : Cantons $=21$. norp $A^{36}=31, w^{1118}$ $=30$. B) norpA is not expressed in Ich5 neurons. The top panel shows the neurons stained with the neuronal marker anti-HRP. The middle panels show the anti-GFP staining. The bottom panels show a merge of both stainings. Although some signal is observed in Ich5 neurons (green arrowheads) when driving GFP using norpA-Gal4, this signal is also observed in the UAS-GFP controls and scolopale cells white arrowheads), indicating some unspecific staining from the anti-GFP antibody. 


\subsubsection{Cilium structure is affected by lack of Rhodopsin}

Data collected previously by Dr. Damiano Zanini showed that localisation of the mechanosensory ion channels in the cilia of Ich5 neurons was aberrant in ninaE $E^{17}$ and $R h 6^{1}$ mutants, as well as mutants with a ninaE knockdown in chos (see appendix, Figure 42). In some Ich5 neurons of mutant larvae, NOMPC would leak into the endolymph space and Inactive would be completely missing. These data suggest that these Rhodopsins may be playing a structural role in chos and that structural defects lead to the locomotion and physiological phenotypes described in the previous sections. To further study the structural roles of Rhodopsins, defects in cell integrity of ninaE $E^{17}$ mutants were assessed with transmission electron microscopy. The images were collected in close collaboration with Nicola Schwedhelm-Domeyer.

Wild-type cilia showed normal organisation, with intact dendrites and ciliary dilation containing electron dense material (Figure 32A). Some of the Ich5 neurons imaged in ninaE ${ }^{17}$ mutants showed a similar normal organisation of the dendrites and cilium as wild-type, with no signs of cilium bending and the electron dense material properly localised (the cilium appears cut most likely due to the preparation) (Figure 32B). However, some strong structural defects were observed in other organs imaged. The defects seen were cilium bending, signs of cilium blebbing and disorganization (Figure 32C, D). In some cases, entire cilium loss with cell material invading the endolymph space was observed, including the electron dense material that is normally found in the cilium dilation (Figure 32D). The fact that not all cilia showed aberrations correlates with Dr. Zanini's findings, where not all the cilia in a single organ ( 5 cilia per organ) showed the mislocalisation of ion channels (see appendix, Figure 42). 
A WT

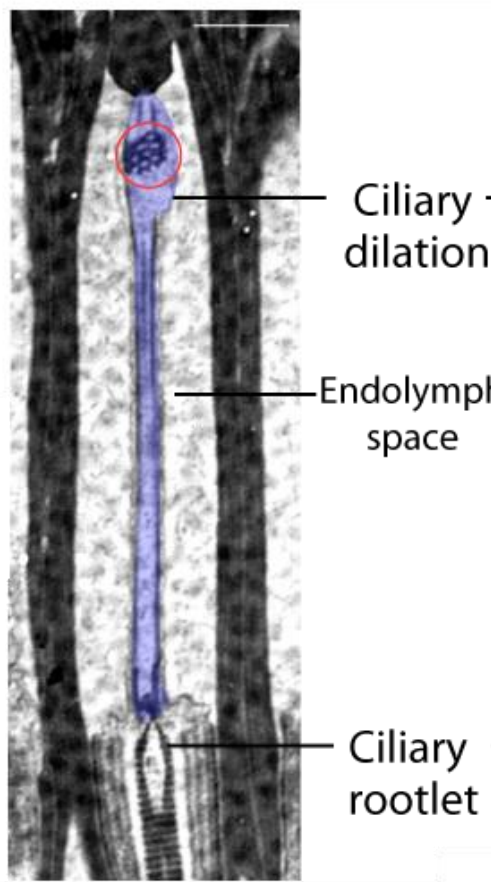

C $\operatorname{nina} E^{17}$

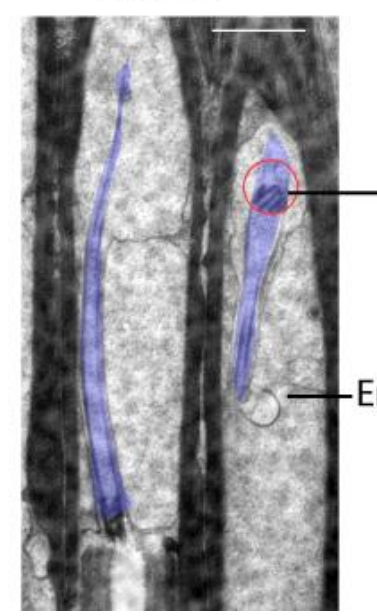

B $\operatorname{nina} E^{17}$
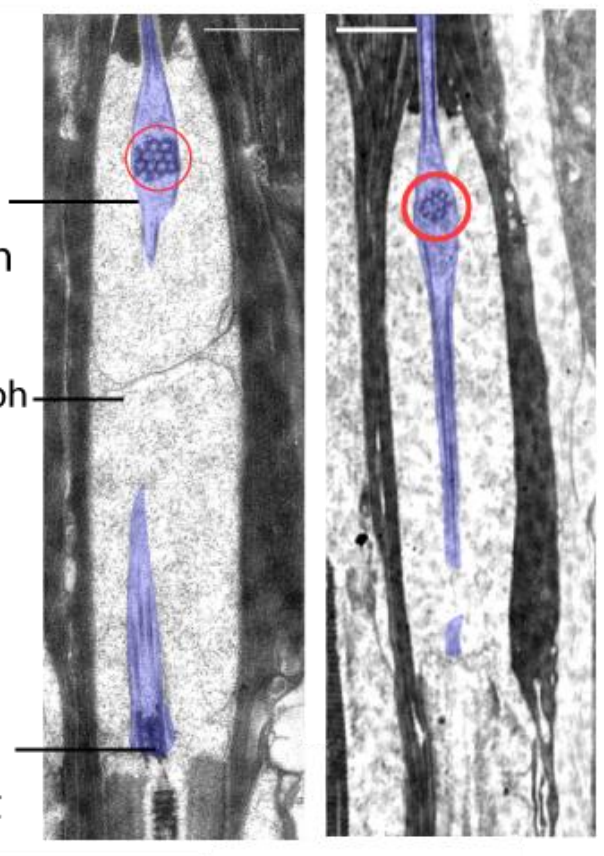

D

$\operatorname{nina} E^{17}$

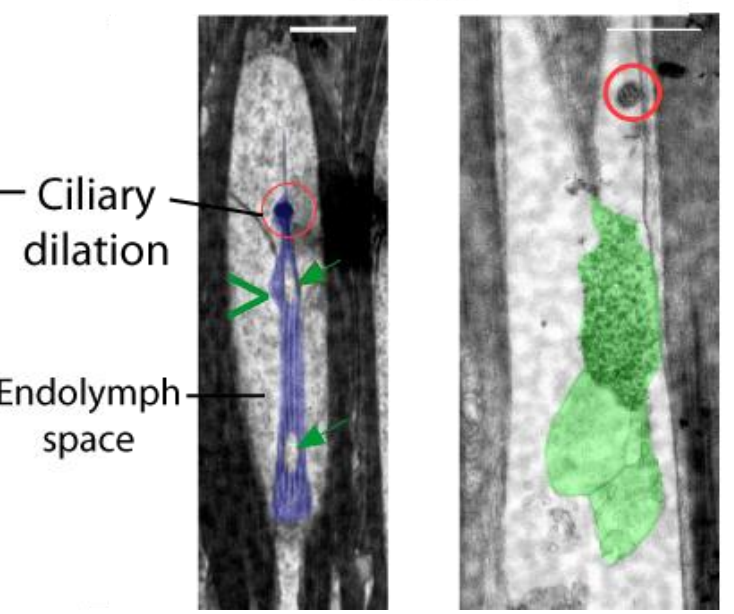

Figure 32. Transmission electron images of Ich5 neuron dendrites. A) Wildtype cilium is highlighted in blue. The electron dense material of the ciliary dilation is circled. The surrounding endolymph space and ciliary rootlet are indicated. B) Cilia of nina $E^{17}$ mutants showing no aberrations. Despite the cilium being cut most likely during the preparation, there is no apparent bending and the electron dense material is properly localised. C) nina $E^{17}$ cilia showing bending. There is no additional aberration in these cilia. D). Strong aberrations in nina $E^{17}$ mutant cilia. The cilium on the left shows signs of blebbing (green arrowhead) and disorganisation (green arrows). The image on the right shows complete cilium loss and the electron dense material of the ciliary dilation leaked to the endolymph space (red circle). Images were obtained from one wild-type and three nina $E^{17}$ mutant organs. Scale bars: $1 \mu \mathrm{m}$. 


\subsection{Discussion}

\subsubsection{Mechanosensory role of opsins}

I studied the role of two opsins, Rh1 and Rh6, in mechanosensation. Both opsins were required for normal locomotion in Drosophila larvae. In addition, they were found to be expressed in proprioceptors in the body wall of the larva, and Rh1 was found to be indispensable for the proper response of these receptors to mechanical stimuli. Previous reports implicated opsins in audition in adults of Drosophila (Senthilan et al., 2012), thermosensation of larvae (Shen et al., 2011; Sokabe et al., 2016), and photosensation in adults and larvae (Montell, 2012; Sprecher et al., 2007). This is the first report of opsins being required for larval mechanosensation.

The algorithm modified from Günther et al., 2016 allowed to obtain data from the crawling and turning phases of larval locomotion. In addition, two different methodologies tested permitted to quantify body contractions successfully. Locomotion was affected in different mechanosensory channel mutants, and larvae with silenced or ablated cho receptors. These findings support the theory that chos are involved in proprioception (Caldwell et al., 2003), which was disputed in one study (Hughes and Thomas, 2007). Locomotion was found to be similarly affected in nina $E^{17}$ and $R h 6^{1}$ mutants. In addition, locomotion defects in $n i n a E^{17}, R h 6^{1}$ double mutants were similar to those of single mutants, indicating that Rh1 and Rh6 function in the same pathway, and both are essential for normal locomotion. This co-requirement of Rh1 and Rh6 is very interesting and unexpected since these two opsins are expressed in different photoreceptor types (Sprecher et al., 2007).

Two wild-type strains were used as controls in the locomotion experiments (i.e., Cantons and $w^{1118}$ ). This was done because some of the strains tested would have either background. Even though $w^{1118}$ had worse locomotor performance in the crawling phases than CantonS larvae, all mutants tested showed a significant difference in all parameters against both wild-type strains. This indicates that although the genetic background may have had some influence in the parameters measured, the mutations had the most significant influence.

The fact that peristaltic contractions were affected, and that a knockdown of ninaE in chos leads to a locomotion deficit, suggest that the phenotype observed comes from a proprioceptive role of opsins in chos, instead of other tissues involved in locomotion like muscles or other bodywall neurons (Grueber et al., 2002; Heckscher et al., 2012; Song et al., 2007). This was also confirmed by immunostainings that show expression of ninaE and Rh6 only in chos and no other body wall tissue (Zanini, Giraldo, et al., 2018). Cho responses to mechanical stimuli in ninaE ${ }^{17}$ 
mutants were reduced but still present, indicating that some cho function is remaining, which explains why the phenotype of $n i n a E^{17}$ and $R h 6^{1}$ mutants is not as severe as that of $n a n^{36 a}$ and $i a v^{1}$ mutants.

Chos have been implicated in both warm sensation (Liu et al., 2003) and cold sensation (Kwon et al., 2010). However, the role of opsins in locomotion was found to be completely independent of their thermosensory role (Shen et al., 2011; Sokabe et al., 2016). Knocking down ninaE in chos did not affect thermosensation, and the locomotion deficits are still evident when larvae are tested at high temperatures outside of the opsin range. Likewise, cells expressing $d t r p A 1$ seem to be dispensable for locomotion, and no dtrpA1 expression is found in chos (Rosenzweig et al., 2005). Only recently a mechanism for opsins in thermosensation has been proposed (Leung and Montell, 2017), but it has not yet been tested. Whether the opsins are temperature sensors, or are integral to the structure of thermosensory cells as found remains to be tested.

The role of Rh6 in thermosensation is dependent on the age of the larvae (Sokabe et al., 2016). Since only late third instar larvae were tested, the role of Rh1 and Rh6 at younger ages remains unclear. Locomotion experiments in first and second instar larvae should be carried out to test if the role of these opsins in proprioception is also age dependent.

\subsubsection{Structural role of opsins}

Electron microscopy images revealed strong structural defects in the cilium of the dendrites of cho neurons. This combined with the data from Dr. Zanini, showing the aberrant distribution of NOMPC and in some cases loss of INACTIVE, points to a role of opsins in sensory dendrite organisation and maintenance (Zanini, Giraldo, et al. 2018). Furthermore, Rh1 and Rh6 localisation was found under the cilia in the dendritic inner segments. These segments contain the ciliary rootlets, which give structural support to the cilia and are essential for cilium maintenance and long-term survival (Yang et al., 2005). Apart from their photosensory role, opsins have been shown to be necessary for photoreceptor structure, and lack of opsins leads to retinal degeneration (Ahmad et al., 2007; Kumar and Ready, 1995; Shieh, 2011). In addition, opsins have been reported to be expressed in thermosensitive neurons in the larval body wall and brain (Shen et al., 2011; Sokabe et al., 2016). Determining if lack of opsins also leads to structural deficits could help to clarify their structural roles. 
How opsins contribute to the structure and maintenance of sensory dendrites is still unclear. They have been implicated in phospholipid translocation between the two membrane leaflets without ATP requirement (Menon et al., 2011). This flippase action of opsins could help shape membrane composition and help maintain dendrite structure, and would explain the structural defects observed in cho cilia.

Interestingly, not all cilia have structural defects and aberrant channel localisation. Other opsins like Rh7 also seem to be expressed in Ich5 neurons (Zanini, Giraldo, et al., 2018), which may mitigate the lack of mutated opsins and keep the structure of some of the dendrites. This could explain why a calcium response to mechanical stimuli is observed in the $n i n a E^{17}$ mutants. Since the microscope resolution used is not high enough to easily separate all five dendrites, the little responses observed may come from those dendrites that have no structural defects. Furthermore, this explains why some movements are observed in the larvae, which lack Rhs, but mechanosensory ion channel or cho neuron ablated mutants cannot move at all.

\subsubsection{Retinal requirement for locomotion}

The light-sensitive retinal chromophore was also found be needed for normal locomotion. Since the experiments were carried out in complete darkness, this requirement seems to be light independent. Interestingly, wild-type larvae that were grown in carotenoid depleted food only showed a strong phenotype in the crawling phases and not the turning phases. Adult flies raised on retinal depleted food and santa-maria ${ }^{1}$ mutants can still have some photoreceptor response, which suggests that some functional Rhodopsin is still present (Wang et al. 2007; Radoslaw Katana, personal communication). The lack of a turning phenotype observed in retinal depleted larvae might indicate a mild phenotype, and that these larvae have better cho function since some retinal is still synthesised. Testing other mutants in the chromophore synthesis pathway (like ninaD, ninaB, or pinta (Pak et al., 2012; Wang et al., 2007)) will allow to further characterise role of retinal in proprioception.

Since the role of opsins in proprioception is independent of light, the retinal requirement is surprising. In photoreceptors, retinal is required for maturation and transportation of Rhodopsin (Ozaki et al., 1993; Wang and Montell, 2007), and lack of retinal leads to a reduction in the opsin levels (Harris et al., 1977). As in photoreceptors, retinal may be needed in proprioceptors for opsin maturation and localisation in the membrane. This could also explain the requirement of retinal in thermosensation, where opsins also play a role independent of light (Shen et al., 2011; Sokabe et al., 2016). 


\subsubsection{PLC requirement for locomotion}

Secondary messenger cascades can help amplify signals received by receptor molecules. Opsins can initiate a signalling cascade that leads to the opening of TRP channels using phospholipase $C$ as an intermediary. The use of PLC as a second messenger has been reported for photosensation and thermosensation (Kwon et al., 2008; Montell, 2012; Shen et al., 2011). The lack of PLC in chos supports the purely structural role of opsins in chos, as suggested by its localisation. Mechanosensory channels are expressed in the cilium where mechanotransduction takes place, but localisation of Rh1 and Rh6 is found in the dendritic inner segments, which serve a more structural than sensory role.

In addition to photoreceptors, PLC is expressed in the brain, thoracic ganglia, legs and abdomen of adult flies (Zhu et al., 1993). Furthermore, it has not only been implicated in various sensory systems like thermosensation (Kwon et al., 2008; Shen et al., 2011), taste (Kim et al., 2010; Masek and Keene, 2013), and olfaction (Riesgo-Escovar et al., 1995) but also renal function (Pollock et al., 2003). A deficit in any of these systems may have contributed to the locomotion defects observed. Additionally, a role in non-cho proprioceptive neurons cannot be excluded. Although a very faint signal was observed by driving GFP using a norpA-Gal4 driver, this faint expression seemed to come from unspecific binding of the anti-GFP antibody, since some signal was also found in the UAS-GFP control line. A tissue specific knockdown of norpA could help confirm whether the phenotype observed in norp $A^{36}$ mutants is due to a role in chos or other tissues.

\subsubsection{Ancient roles of opsins}

Metazoan opsins can be divided into four major groups: ciliary opsins (c-opsins), rhabdomeric opsins (r-opsins), RGR/Go-opsins, and placopsins (Feuda et al., 2012). Although c-opsins and ropsins are found in vertebrates and invertebrates (Figure 33), all Drosophila opsins are r-opsins (Shichida and Matsuyama, 2009). The expression of an r-opsin in ciliated cell like cho neurons is novel and unexpected.

C-opsins and r-opsins diverged before the appearance of bilaterians (Figure 33) (Feuda et al., 2012). Given the structural roles of opsins found in ciliary cells and rhabdomeric cells (Kumar and Ready, 1995; Shieh, 2011), and that photoreceptor cells and chos evolved from the same cell type (Niwa et al., 2004), it seems that opsins had an ancestral structural role for dendrite maintenance, and their photosensory roles in rhabdomeres evolved later on. Opsins have also been found to have mechanosensory functions in the auditory system of adult flies (Senthilan et al., 
2012), which is also mediated by chos (Eberl, 1999). It seems that adult fly hearing took over its opsin dependence from proprioception since adult insect hearing organs differentiated from the serial set of proprioceptive chos (Göpfert and Henning, 2016). However, whether the chos in the adult ear require opsins for structural purposes remains to be shown.

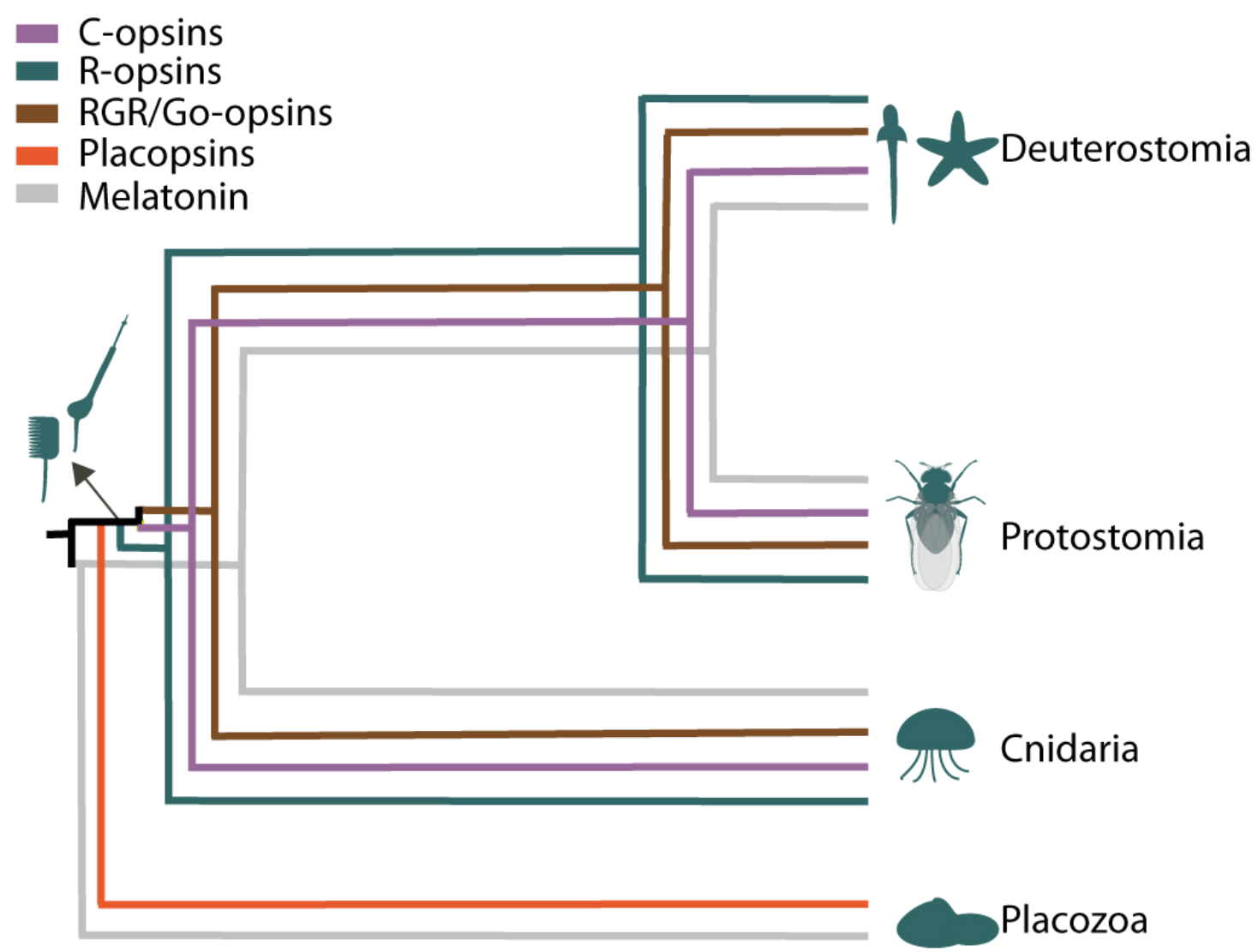

Figure 33. Metazoan opsin evolution scheme after Feuda et al., 2012. The sister group of the opsin family is melatonin. Placozoans have a unique group of that shares deep homology with the other group of opsins referred to as Placopsins. R-opsins diverged from c-opsins and RGR/Go-opsins before the appearance of bilaterians (indicated by the arrow). Finally, c-opsins diverged from RGR/Go-opsins before the appearance of bilaterians as well. Modified from Feuda et al., 2012 and Dr. Bart R. H. Geurten.

Opsins are the main photoreceptor molecule in vertebrates and invertebrates and have also been implicated in thermosensation. Studying structural roles of opsins in vertebrate photoreceptors and Drosophila thermosensitive neurons that are opsin dependent could help to understand the role of opsins in dendrite maintenance further and provide more evidence towards the ancestral structural roles of opsins. 


\subsection{Conclusions}

Two opsins, Rh1 and Rh6, were found to be expressed in chos and to be essential in larval proprioception and normal mechanically evoked calcium responses of cho neurons. These roles were independent of light but still required a functional chromophore, most likely for the normal localisation of the opsins in the membrane. Structural defects in the cilia of chos lacking these two opsins were found, suggesting that these opsins are required for dendrite maintenance. Lack of PLC suggests that the role of opsins in chos is not associated with a signalling cascade, and most of the evidence obtained so far points to a purely structural role. In addition to photoreceptors, chordotonal proprioceptors seem to be the second animal cells whose structure and function are opsin dependent. Since chos and photoreceptors presumably evolved from the same cell type (Niwa et al., 2004), it is possible that their common ancestor cells already used opsins for dendrite maintenance. From the evidence provided for Drosophila photo- and chordotonal receptors, it seems that structural r-opsin functions might have been conserved throughout the evolution of the different types of sensory receptor cells, which could explain the dependence of diverse sensory modalities on opsins. 


\section{Chapter 3}

\section{Larval chordotonal organs in thermosensation}

\subsection{Introduction}

Larval chordotonal organs (chos) provide proprioceptive feedback during locomotion and are essential for proper crawling behaviour (Caldwell et al., 2003) (see Chapter 2). Furthermore, they serve as touch receptors (Caldwell et al., 2003) and vibration receptors (Fosque et al., 2015; Zhang et al., 2013). In addition to their mechanosensory roles, chos have been implicated in temperature sensation (Kwon et al., 2010; Liu et al., 2003; Scholz et al., 2017). However, the evidence for the thermosensitive role of larval chos is contradicting, and whether they serve a role in warm sensation or cold sensation is not clear.

\subsubsection{Chordotonal organs and thermosensation}

In 2003 Liu et al. did a study of the thermosensitive neurons of Drosophila larvae by measuring the physiological response of neurons to cold and hot stimuli using the ratiometric calcium sensor camaleon 2.1. In their study, they characterise the cold receptors in the terminal organ and hot and cold receptors in the body wall of the larva. They found that pentameric cho (Ich5) neurons in the body wall respond to heating. However, this response was very low compared to other body wall neurons (Figure 34), but it was enough for the authors to conclude that Ich5 neurons mediate thermosensation. In addition, it should be noted that the rise in temperature was of $22^{\circ} \mathrm{C}$, and this only lead to a very small signal (Figure 34). Whether the signal is an actual response to temperature, or just an increase in the spontaneous activity of the neurons by the increase in temperature is not clear. Although the evidence provided for the role of Ich5 in warm thermosensation by this study is very poor, larval chos are still cited as being involved in warm temperature sensation (Scholz et al., 2017). Even though better calcium sensors have been discovered since (Chen et al., 2013), no 
additional study has focused on testing the responses of Ich5 to warm temperatures using more sensitive calcium indicators. Additionally, behavioural studies have shown that chos are not involved in the avoidance of warm temperatures, and no expression of the heat sensing channel dTRPA1 in chos has been found (Kwon et al., 2010; Rosenzweig et al., 2005).

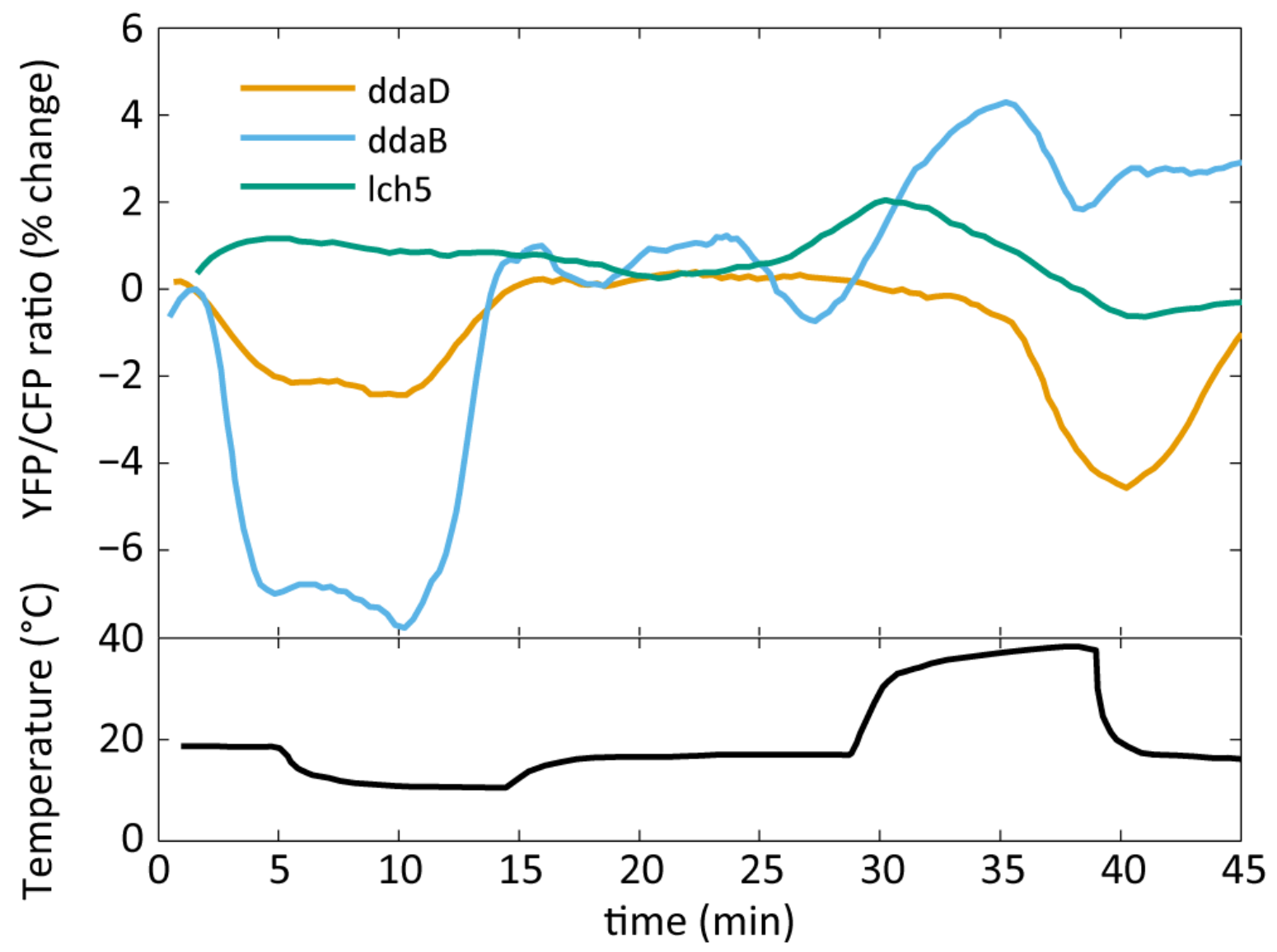

Figure 34. Camaleon fluorescence of body wall neurons modified after Liu et al., 2003. The responses of body wall neurons of Drosophila larvae were imaged using camaleon 2.1. A strong change to cooling can be observed in ddaA and ddaB neurons. A very small increase can be observed in Ich5 neurons. ddaB neurons also showed a strong response to heating, whereas Ich5 show only a very small response. It should be noted that the step in the hot stimulus was of $22^{\circ} \mathrm{C}$.

Better evidence for the role of chordotonal organs in warm sensation has been found in Drosophila adults. Femoral chordotonal organs $(\mathrm{fco})$ in the leg of the adults were found to mediate circadian entrainment to warm temperatures, mediated by the ionotropic receptor IR25a (Chen et al., 2015). These organs responded to warm temperatures, and ectopic expression of IR25a conferred thermosensitivity to non-thermosensitive cells (Chen et al., 2015). However, IR25a mediates cold sensation in larvae ( $\mathrm{Ni}$ et al., 2016), and expression in larval chos has not been reported. 
In their study of the physiological responses to temperature in 2003 , Liu et al. also found that Ich5 neurons responded to cold temperatures. However, the response to the cold stimulus was even lower than the response to hot stimuli (Figure 34) (Liu et al., 2003). No additional studies have focused on the physiological response of Ich5 neurons to cold stimuli. Nonetheless, Kwon et al. in 2010 found that larvae that lack the channel Inactive expressed in cho neurons were unable to avoid cold temperatures. In addition, larvae with impaired chos also lost cold avoidance behaviour. In contrast, warm avoidance was not impaired in larvae with silenced chos or larvae lacking Inactive, suggesting that chos are not involved in warm sensation and contradicting previous findings (Liu et al., 2003). This contradiction, however, is not discussed by Kwon et al., and different studies refer to larval cho as either warm sensors or cold sensors, without addressing the issue (Barbagallo and Garrity, 2015; Garrity et al., 2010; Kavlie and Albert, 2013; Li and Gong, 2016; Rosenzweig et al., 2005; Scholz et al., 2017; Sokabe et al., 2016; Turner et al., 2016).

\subsubsection{Candidate molecules for thermosensation in larval chos}

Chos express three mechanosensory channels: NOMPC, Inactive and Nanchung (see Chapter 2) (Cheng et al., 2010; Gong et al., 2004). NOMPC has been found to contribute to the sensation of noxious cold temperatures, but this function seems to be restricted to Class III multidendritic neurons (MD neurons) in the dorsal part of the larval body wall (Turner et al., 2016). Furthermore, although Inactive channels are essential for cold avoidance, they do not seem to be activated directly by temperature (Kwon et al., 2010), suggesting that if chos are involved in thermosensation, other molecules should be involved.

Brivido is a channel expressed in the three cold sensing neurons of the adult arista (Gallio et al., 2011). Three Brivido proteins have been characterised (Brivido1-3) which are encoded by the genes brv1, brv2, and brv3 respectively (Gallio et al., 2011). Although they share homology with TRPP channels, they have ten transmembrane domains instead of the six found in TRP channels, and it is still debated whether they belong to the TRP channel superfamily (Barbagallo and Garrity, 2015; Fowler and Montell, 2013). Brivido channels do not seem to respond to temperature but are essential for the avoidance of cold temperatures and the physiological response of cold sensing neurons in the arista (Gallio et al., 2011). How they contribute to cold sensation is not yet clear. 
In larvae, the cold sensors in the dorsal organ, require IR25a and IR21a for cold sensation, but Brivido is dispensable for dorsal organ neuron responses to cold stimuli ( $\mathrm{Ni}$ et al., 2016). Nonetheless, at least Brivido1 seems to be required for the avoidance of cold temperatures, suggesting Brivido channels may act in a different population of cold cells (Ni et al., 2016). Brivido1 is also expressed in the adult Johnston's organ, but whether it plays a thermosensory or mechanosensory role in this organ has not be examined (Gallio et al., 2011). However, Brivido expression in larval chos has not been studied. The study of Brivido in larval chos could help to clarify if and how chos contribute to cold sensation.

Two thermosensory channels for heat detection have been characterised in Drosophila: dTRPA1 and GR28b(D) (Mishra et al., 2018; Ni et al., 2013; Rosenzweig et al., 2005). dTRPA1 has been found to be involved in thermosensation in both larvae and adults (Hamada et al., 2008; Rosenzweig et al., 2005), but its role seems to be independent of chos (Kwon et al., 2010; Rosenzweig et al., 2005). GR28b(D) belongs to the gustatory receptor (GR) family, which is a family of 68 proteins (see (Montell, 2009, 2013) for reviews). Although the first members of the family were gustatory receptors (hence the family name), some of the receptors in the family are involved in other senses like thermosensation (Mishra et al., 2018; Ni et al., 2013), photosensation (Xiang et al., 2010), and $\mathrm{CO}_{2}$ detection (Kwon et al., 2007; McMeniman et al., 2014). A member of the GR28B family, GR28B(D), is an example of a GR involved in thermosensation. GRs have seven transmembrane domains and, even though there is evidence suggesting that gustatory receptors serve as GPRCs (Xiang et al., 2010), activation of GR28B(D) by temperature indicates that it is a cation channel (Mishra et al., 2018; Ni et al., 2013). In larvae, GR28B serves as a light sensor necessary for the avoidance of high intensity light (Xiang et al., 2010). Whether Gr28B(D) serves a thermosensory role in chos has not been analysed.

If larval chos are involved in thermosensation, it would be expected that they express at least one of the thermosensory molecules that have been characterised in adult thermosensors, or other thermosensory cells in the larva. Identification of such molecules would help understand the role of chos in thermosensation, and whether they play a role in hot or cold sensation.

\subsubsection{Chapter overview}

In this chapter I, studied the expression of candidate thermosensory molecules in Ich5 of larvae. Furthermore, I assessed the role of chordotonal organs in thermosensation using the photoconvertible non-reversible calcium sensor CaMPARI. In addition, using behavioural assays I studied the role of Brivido in thermosensation in larvae and larval chordotonal organs. 


\subsection{Results}

\subsubsection{Thermosensory channel expression in chos}

The expression of three molecules involved in thermosensation - brv1, Ir25a, and Gr28b - was assessed in Ich5 neurons. For this purpose, GFP expression was driven by driving Gal4 using promotors for each of the genes studied. The nervous system was stained using conjugated donkey anti-HRP (1:300), and the GFP signal was amplified with an anti-GFP staining (chicken anti-GFP primary antibody (1:1000) and Alexa Fluor 488 anti-chicken secondary antibody (1:300)). Confocal images of the larval body wall revealed that brv1 is expressed in at least five sensory neurons. Two of these neurons is a subset (neurons 1 and 3) of the five neurons in the Ich5 (Figure 35A, B). Additionally, the IdaB near the Ich5 also showed strong GFP expression (Figure 35B). These neurons have a high degree of branching and have been implicated in touch sensation (Grueber et al., 2002; Tsubouchi et al., 2012). Furthermore, the neuromuscular junction in the lateral section of the larval body wall was positive for GFP (Figure 35B).

Two neurons in the dorsal cluster of sensory neurons were also GFP positive. One of these neurons is the bipolar dendrite md neuron dbd (Figure 35C, D). It has been reported that this neuron expresses dTRPA1 and Rh1, and is involved in warm thermosensation (Shen et al., 2011). In addition, a dorsal md neuron also expressed GFP under the control of brv1-Gal4 (Figure 35D). This neuron seems to be the Class III ddaF neuron in the dorsal cluster of da neurons (Figure 35C). As found in the lateral section, the neuromuscular junction in the dorsal section were also GFP positive (Figure 35D).

Although the channel IR25a is expressed in adult femoral chos (Figure 36C), no expression of IR25a was found in larval Ich5 (Figure 36A). Instead, two neurons of the external sensory organ v'es2 expressed GFP under the control of Ir25a-Gal4 (Figure 36A) (stainings and images were done by Dr. Damiano Zanini). In addition, no expression of the warm thermosensitive channel GR28B(D) was observed in Ich5 neurons or any other neighbouring neuron (Figure 36B). The functionality of the Gal4 lines was confirmed by measuring GFP expression in fco (for Ir25a-Gal4) and the adult arista (for Gr28b.d-Gal4) (Figure 36C, D). These findings support a role of larval chos in cold sensation and a possible role of Brivido1 in them. 
A

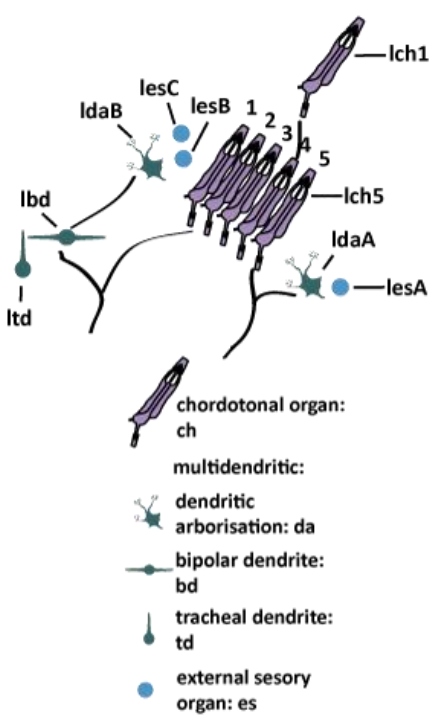

C

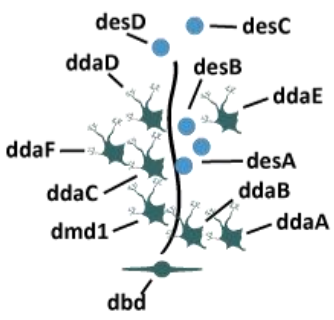

B
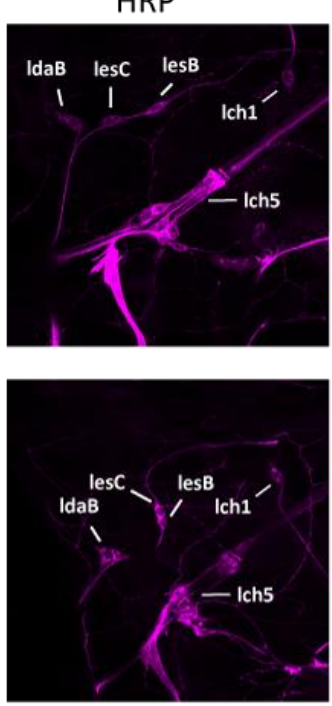

D

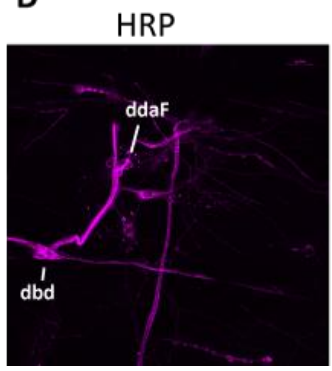

GFP
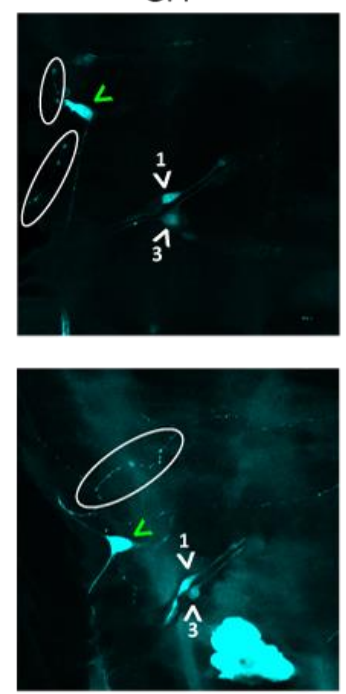

GFP

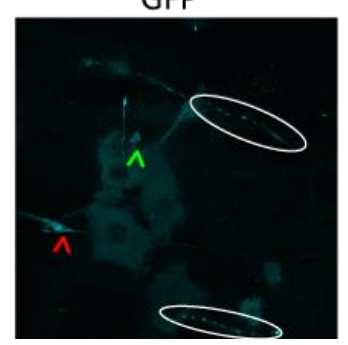

Merge
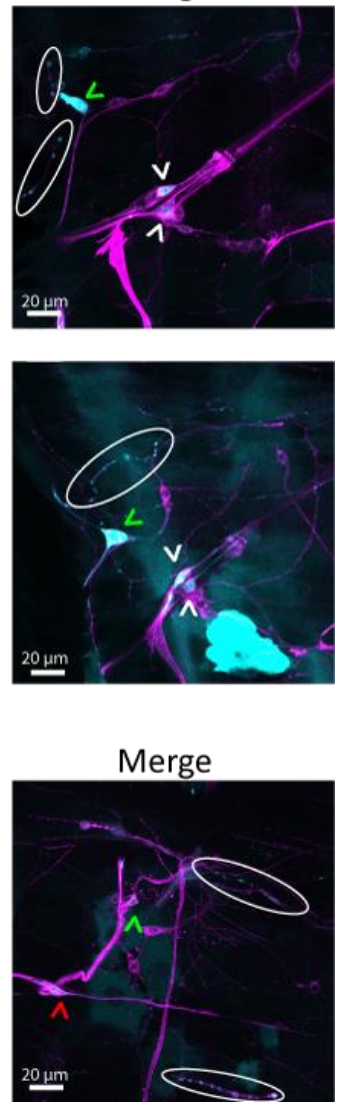

Figure 35. brv1 expression in body wall neurons. A) Schematic of the lateral cluster of body wall neurons of larvae. The number of the five neurons of the Ich5 is depicted. B) brv1 expression in the lateral body wall neurons. The left panel shows the neurons stained with the neuronal marker anti-HRP, the middle panels the GFP signal (amplified by anti-GFP staining) driven by brv1-Gal4, and the right panels show a merge of both stainings. brv1 is expressed in neurons 1 and 3 of the Ich5 (white arrowheads). Additional expression is found in $\mathrm{ddaB}$ (green arrowhead) and the neuromuscular junction (white ovals). C) Schematic of the dorsal body wall neurons of larvae. D) Panels represent the same as B. brv1 is expressed in the dbd neuron (red arrowhead) and ddaF (green arrowheads), and the neuromuscular junction (white ovals). 
A

HRP

GFP

Merge
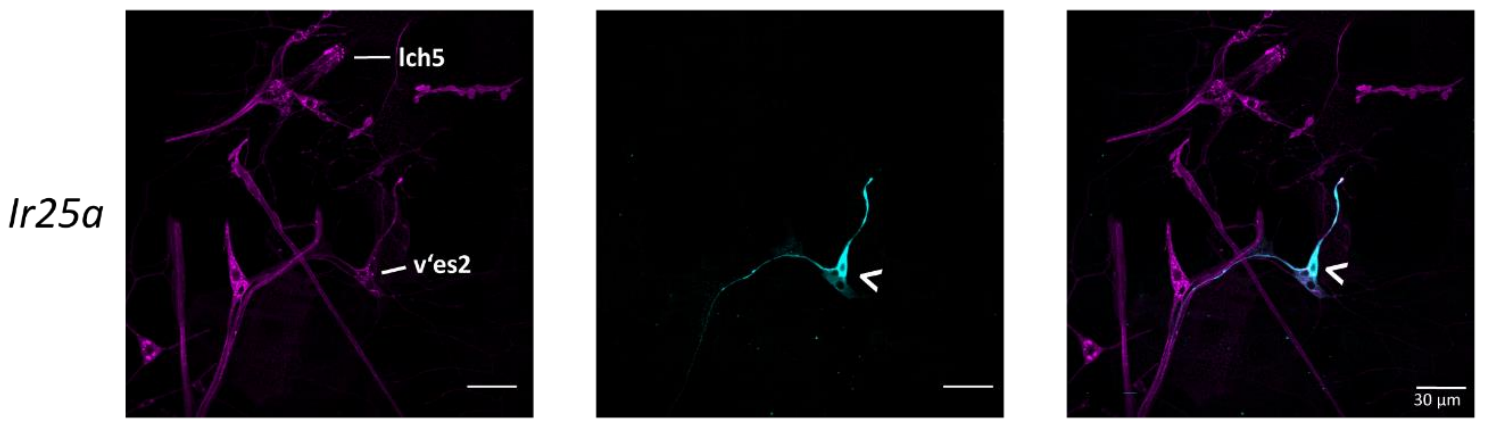

B

HRP

GFP

Merge
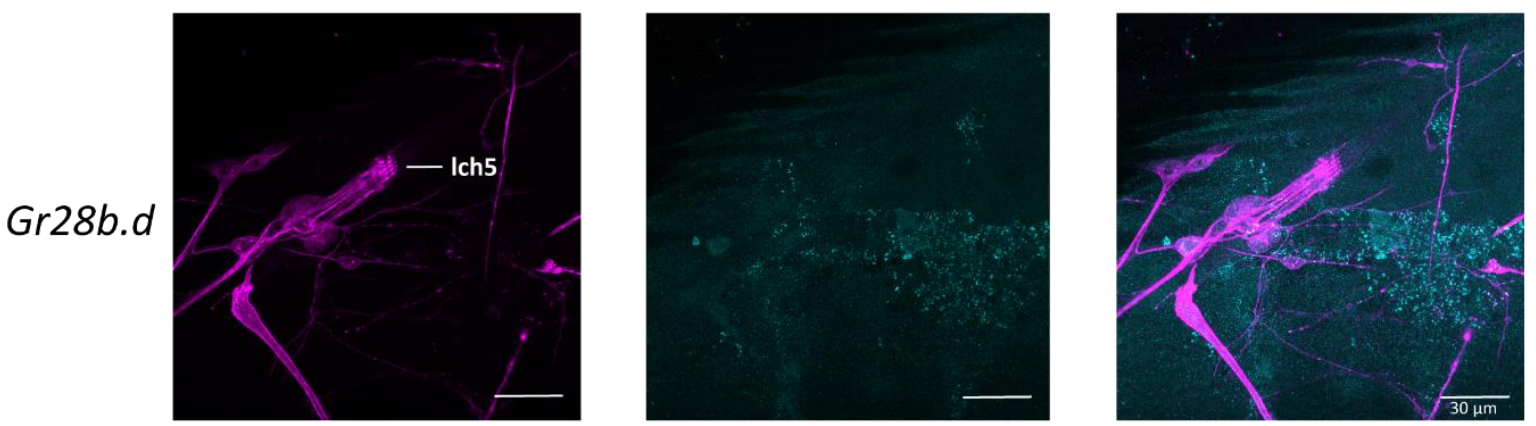

C

Ir25a

D

Gr28b.d

adult fco

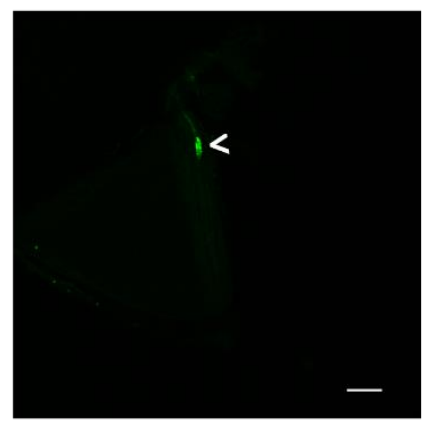

\section{adult arista}
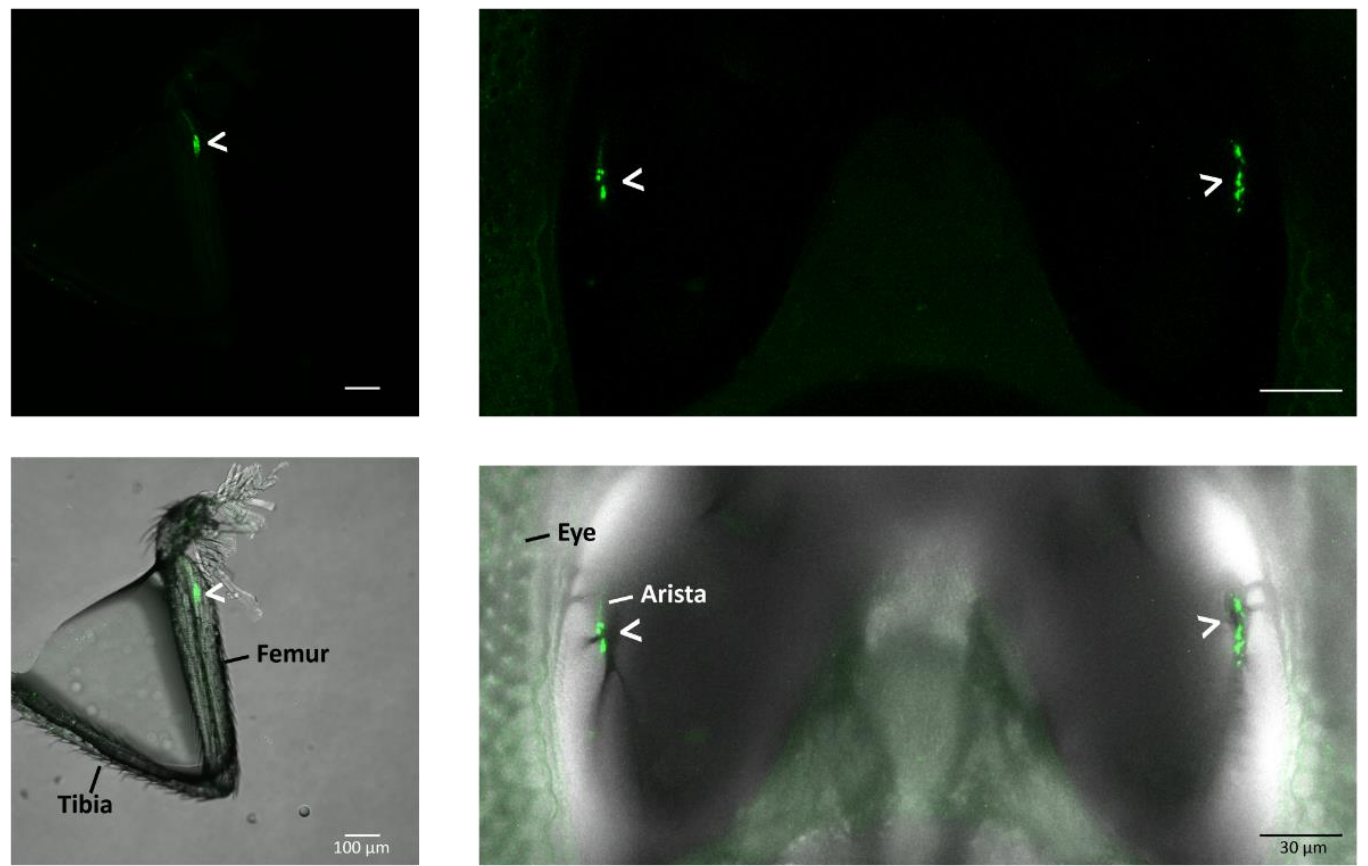

Figure 36. Expression of Ir25a and Gr28b.d. A-B) The left panel shows the neurons stained with the neuronal marker anti-HRP, the middle panels the GFP signal amplified with anti-GFP staining, and the panel on the right the merge of both stainings. A) Expression of Ir25a in larval body wall neurons. Ir25a is expressed in the ventral es v'es2 (white arrowhead), but not in Ich5 neurons. The images were obtained by Dr. Damiano Zanini. B) Gr28b.d is not expressed in Ich5 or neighbouring neurons. C) Expression of Ir25a in the adult femoral chordotonal organ (white arrowheads) confirms that the IR25a-Gal4 used is functional. D) Expression of Gr28b.d in the adult arista (white arrowheads) confirms that the Gr28b-Gal4 line used is functional. 


\subsubsection{Brivido1 is not required in chos for thermosensation}

Brivido1 was previously found to be essential for cold thermosensation in larvae, but it was not required in the dorsal organ neurons that sense cold ( $\mathrm{Ni}$ et al., 2016). To assess a possible thermosensory role of Brivido1 in chos, a cho specific knockdown of brv1 was done by driving brv1RNAi using dnai2-Gal4. Temperature preferences of larvae were assessed in a Peltier elementbased setup as described in section II.III.IV.II. Larvae were placed in the gradient ranging from $12.5^{\circ} \mathrm{C}-28^{\circ} \mathrm{C}$ for 10 minutes and their final temperature distribution was measured resulting in a single distribution per strain. The final distribution of the larvae was corrected for cold trapping using IGLOO (section II.V.IV and Chapter 1). The 95\% confidence interval at each temperature of the gradient was calculated per strain to obtain the final corrected preferences.

Larvae of both parental lines and the line carrying the chos knockdown of brv1 distributed between $15^{\circ} \mathrm{C}-19^{\circ} \mathrm{C}$ (Figure $37 \mathrm{~A}-\mathrm{C}$ ). Only a strong avoidance of $12.5^{\circ} \mathrm{C}$ and no strong avoidance of hot temperatures was observed. The median corrected preferences were around $17^{\circ} \mathrm{C}$ for all lines (Figure 37D), indicating that a knockdown of brv1 in chos does not lead to a thermosensory deficit, and brv1 does not play a thermosensory role in larval chos.

\subsubsection{Chordotonal organ responses to temperature}

Brivido channels have been implicated in cold thermosensation in the adult fly. Expression of brv1 in a subset of cho neurons in the larval body wall supports the role of chos in cold sensation. In addition, it suggests that a subset of neurons may be playing a thermosensory role, while the rest of the organ plays a mechanosensory role. To determine if chos, in fact, respond to temperature, CaMPARI (see section II.X.II.) was expressed in chos using the cho specific Dhc93-Gal4 driver (Figure 38A). Late third instar wandering larvae were placed on a Peltier element (PE) in a dark box (see section II.II.IV.) that was set to $18^{\circ} \mathrm{C}$. Once the larva was on the PE, the door of the box was closed so the larva was in complete darkness. Then, the UV-light needed for CaMPARI photoconversion from green to red was switched on, and the temperature of the PE was dropped to $13^{\circ} \mathrm{C}$ (cool) or raised to $23^{\circ} \mathrm{C}$ (warm). The cool temperature was in the range suggested for cho thermosensation (Kwon et al., 2010), and the warm temperature was in the range of temperatures suggested for Rhodopsins (Shen et al., 2011). The time for the temperature to reach the final value was around 30 seconds, and the photoconversion light was kept on for an extra 30 seconds. For control experiments, the larvae were kept at $18^{\circ} \mathrm{C}$ (constant) for 1 minute. Larvae were tested individually or in groups of two and were dissected and imaged as described in section II.X.I. 
A

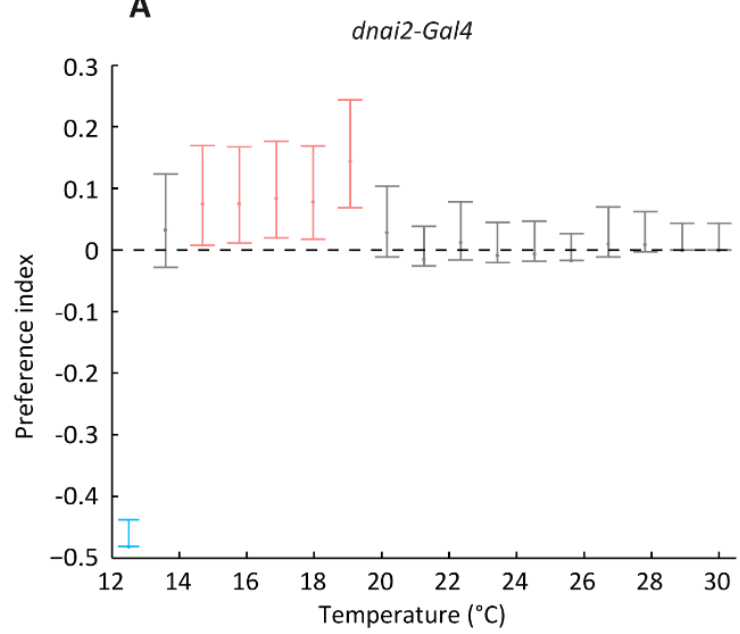

C

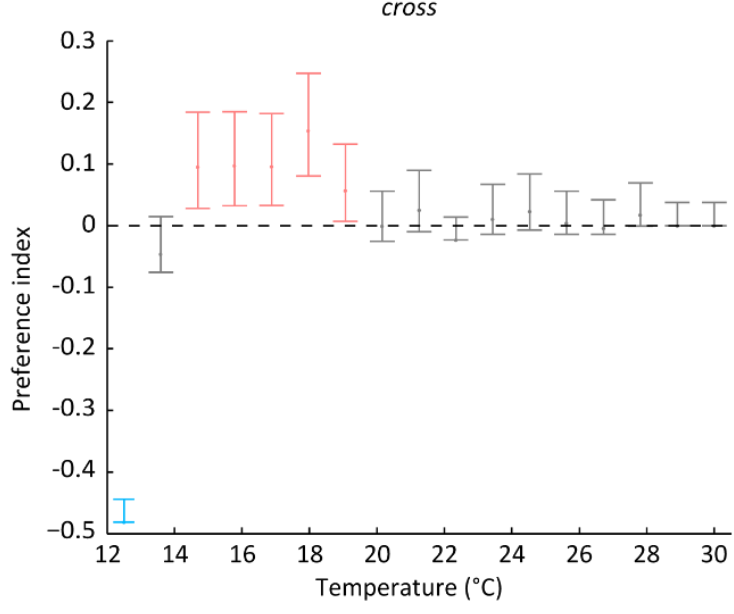

B

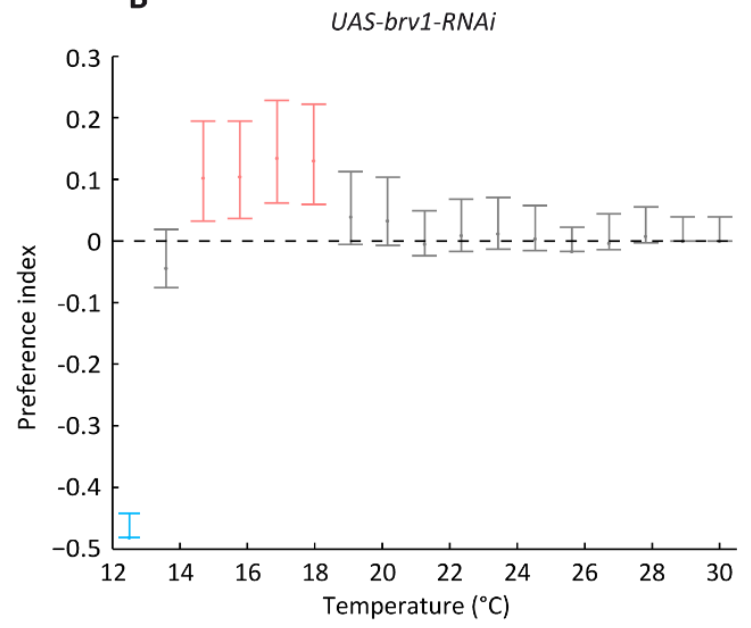

D

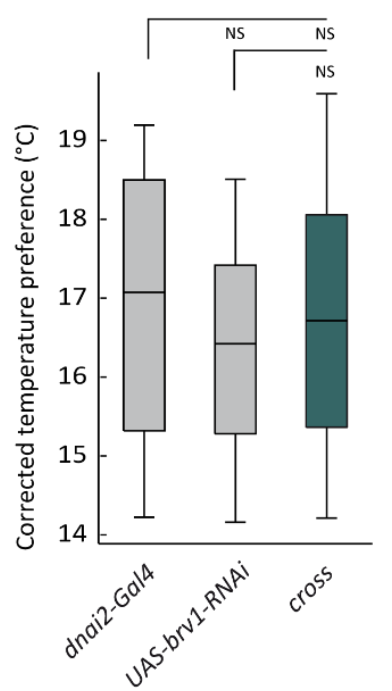

Figure 37. Temperature preferences of larvae with a cho specific brv1 knockdown. A knockdown of brv1 in cho neurons by driving RNAi for ninaE in chos using the cho specific driver dnai2-Gal4. A-C) Cold trapped corrected distribution of larvae in a temperature gradient. After the correction (see chapter 1), a preference index is obtained for each temperature. A negative preference index (blue) means the temperature is avoided and a positive index (red) means the temperature is preferred. The points represent the median and the bars represent the $95 \%$ confidence interval. If the $95 \%$ confidence interval overlaps with zero (grey) the temperature is considered as neither preferred nor avoided. A) Gradient distribution of dnai2-Gal4. B) Gradient distribution of UAS-brv1-RNAi. C) Gradient distribution of the cross of A and $B$. D) Temperature preferences of the corrected distributions in A-C. Only the temperature values in the preferred temperatures (i.e., red points in $\mathrm{A}-\mathrm{C}$ ) are used to calculate the preference. The black line indicates the median, the box marks the lower and upper quartile, and the whiskers the range of entire data set in the case where there are no outliers, and in the case outliers are present the 1.5 interquartile distance; and the outliers are indicated by black crosses. Box colours D: grey $=$ Gal4 and UAS controls and green $=$ cross. Significant differences from parental lines (Fisher's exact permutation tests with Benjamini - Hochberg correction): ${ }^{*}=p<0.05,{ }^{* *}=p<0.01,{ }^{* * *}=p<0.001$, NS = not significant. dnai2-Gal4 $\mathrm{n}=83$, UAS-brv1-RNAi $\mathrm{n}=93$, cross $\mathrm{n}=97$. 
The $\mathrm{F}_{\text {red }} / \mathrm{F}_{\text {green }}$ ratio of the whole organ (cell bodies and dendrites) was quantified to measure the cho activity during stimulation (temperature and UV-light). No difference was observed between cool stimulated larvae and controls when every organ measured was taken into account (Figure 38B), or when the median ratio per larva was compared (Figure 38C). The same was observed for warm stimulated larvae (Figure 38B, C).

Since brv1 seems to be expressed in only a subset of cho neurons (i.e., 1 and 3, Figure 35B), it is possible that only those neurons respond to temperature stimulation. In the images where all five cho neurons could be easily identified, the ratio of each dendrite was calculated to determine differences in the response of each neuron. No difference between controls and temperature stimulated larvae was observed for neurons 1 and 3 (Figure 38D, E respectively). These data suggest that chos do not respond to warm or cool stimulation.

The lack of responses to temperature stimulation observed could also be explained by an ineffective photoconversion of CaMPARI. To test that the UV-light stimulation was enough for CaMPARI photoconversion, the $\mathrm{F}_{\text {red }} / \mathrm{F}_{\text {green }}$ ratio of organs of larvae that were not exposed to UVlight was calculated. These organs had a significantly lower ratio than organs at constant temperature and exposed to UV-light (Figure 38B), and almost no red fluorescence was observed. These data suggest that Ich5 neurons did not respond to cool or warm stimulation, and the photoconversion observed was due to Ich5 neuron activity due to its proprioceptive function and their spontaneous activity. More importantly, it confirms that the UV-light used during the experiments was strong enough to generate CaMPARI photoconversion. 
A

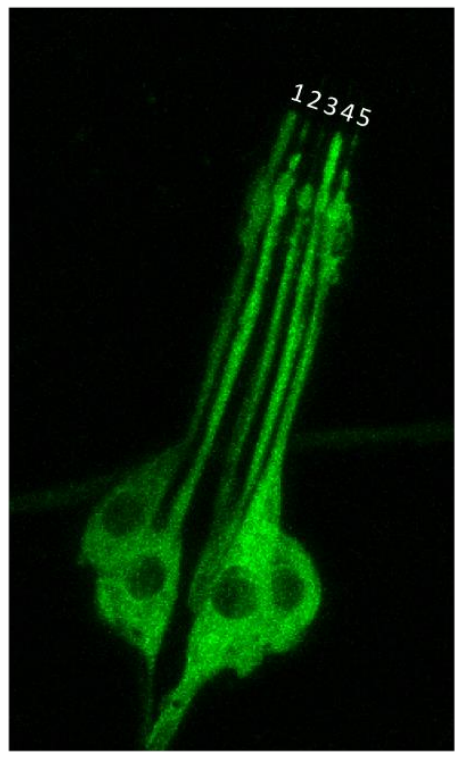

Figure 38. CaMPARI imaging of Ich5 neurons stimulated with temperature. A) Confocal image of Ich5 neurons expressing green CaMPARI. Each Ich5 neuron is numbered (1-5). B) $F_{\text {red }} / F_{\text {green }}$ ratio of Ich5 neurons of larvae stimulated with cool and warm temperatures, or kept at a constant temperature. The ratio of Ich5 neurons that were not stimulated with UV-light indicate that the light stimulus was appropriate for CaMPARI green to red photoconversion. C) Median $\mathrm{F}_{\text {red }} / \mathrm{F}_{\text {green }}$ ratio of each larva stimulated from $A$. D) $F_{\text {red }} / F_{\text {green }}$ ratio of only neuron 1 dendrites. E) $\mathrm{F}_{\text {red }} / \mathrm{F}_{\text {green }}$ ratio of only neuron 3 dendrites. The data is represented as in Figure 37. Significant differences from constant temperature stimulation control (Fisher's exact permutation tests with Benjamini - Hochberg correction): ${ }^{*}=p<0.05,{ }^{* *}=p<$ $0.01,{ }^{* * *}=\mathrm{p}<0.001, \mathrm{NS}=$ not significant.
B

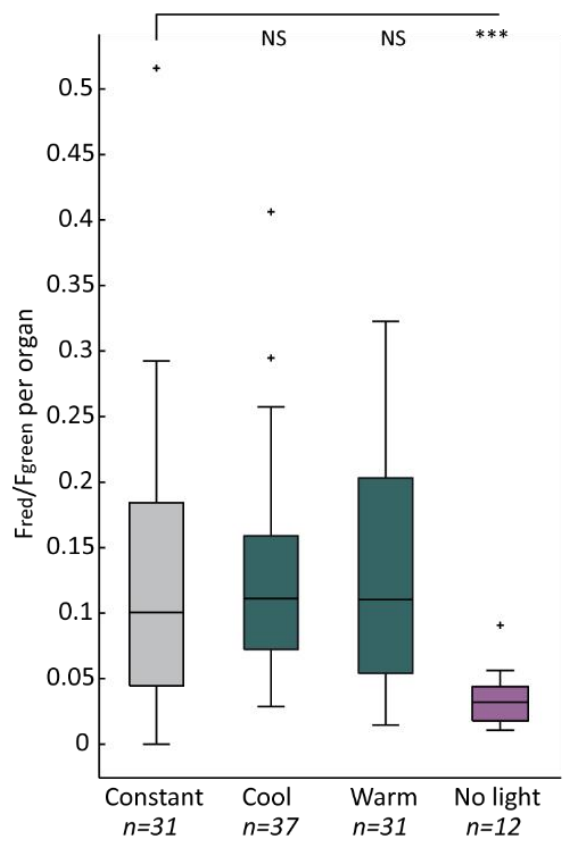

D

Neuron 1

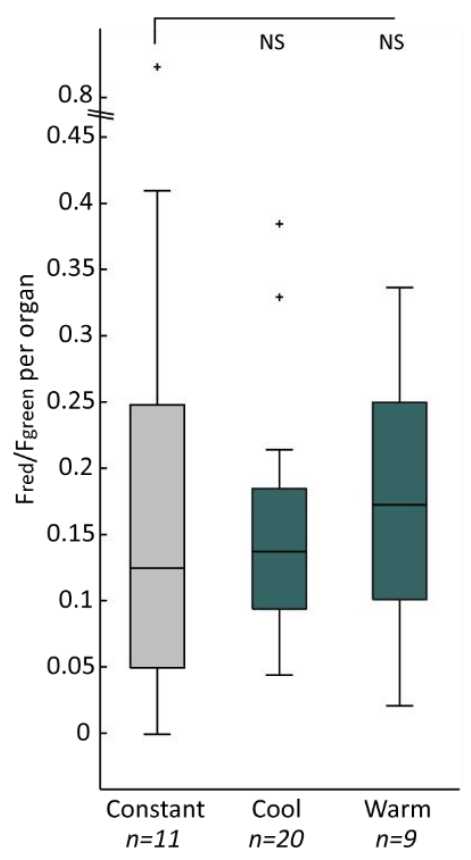

C

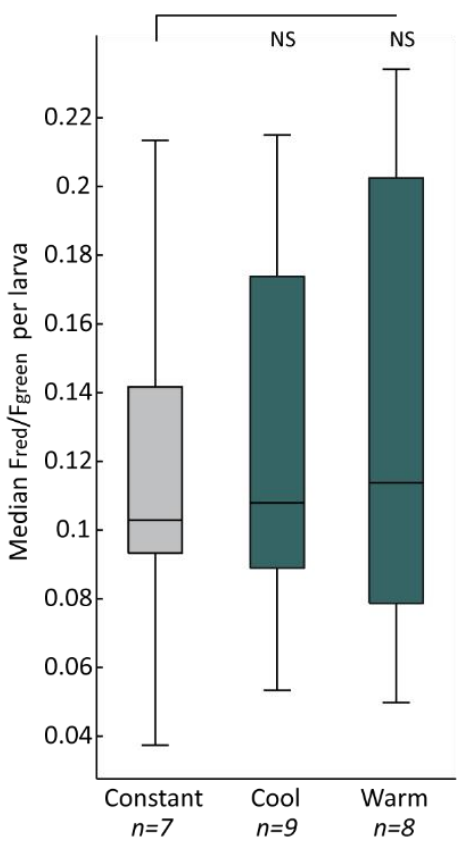

E

Neuron 3

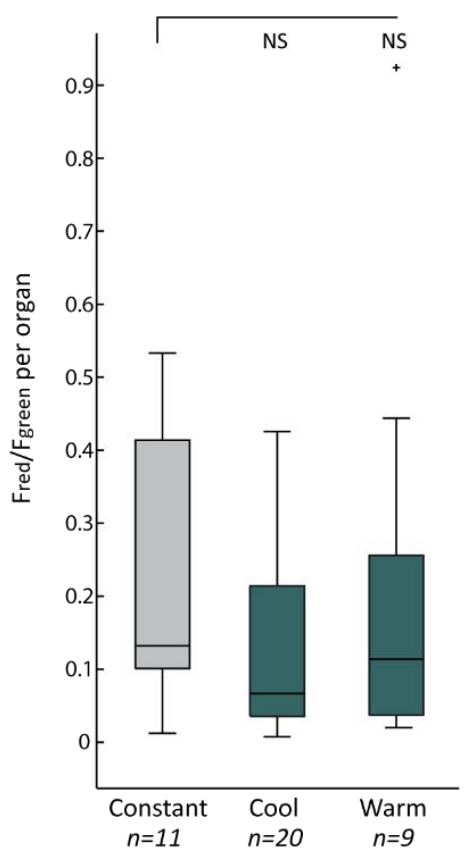




\subsubsection{Brivido1 is not required in chos for locomotion}

Expression of brv1 in Ich5 neurons indicates that it may be serving a mechanosensory role. Since a brv1 mutant was not available, a knockdown of brv1 in chos was done using the same lines as is section 3.2.2. Locomotor performance of larvae was studied using the assay described in sections II.III.I. and II.VI.I. The larvae with a cho knockdown of brv1 did not show any locomotion deficits when compared to the UAS-brv1-RNAi parental line (Figure 39A), indicating that brv1 is not required in chos for proper locomotion and that the proprioceptive role of chos is not affected by lack of Brivido1.

Brivido2 is expressed in the same neurons as Brivido1 and seems to play a redundant role in Drosophila adults (Gallio et al., 2011). Although no expression data for brv2 in larvae is available, it is possible that in larvae these two channels are also coexpressed in larvae and serve redundant roles. Locomotor performance of brv2 ${ }^{\text {M104916 }}$ mutants was tested. The only parameter affected by lack of Brivido2 was the turning frequency (Figure 39B). This could be due to a problem in thermosensation since larvae that lack dTRPA1 sensing neurons were also found to have increased turning frequency, but do not have crawling deficits (see Chapter 2, section 2.2.3).

To confirm a thermosensitive role of Brivido2 in thermosensation, temperature preferences of $b r v 2^{M 104916}$ mutant larvae were tested in a temperature gradient ranging from $12.5^{\circ} \mathrm{C}$ $-28^{\circ} \mathrm{C}$ and corrected for cold trapping as described in section 3.2.2. Wild-type controls distributed in the gradient between $18^{\circ} \mathrm{C}-21^{\circ} \mathrm{C}$ (Figure 39C) while brv2 ${ }^{\text {MI04916 }}$ mutants had a distribution at lower temperatures between $15^{\circ} \mathrm{C}$ and $18^{\circ} \mathrm{C}$ (Figure 39D). The corrected preferred preferences were significantly reduced in brv2 ${ }^{\text {MI04916 }}$ (Figure 39E) indicating that Brivido2 plays a role in cold thermosensation, but not proprioception. 


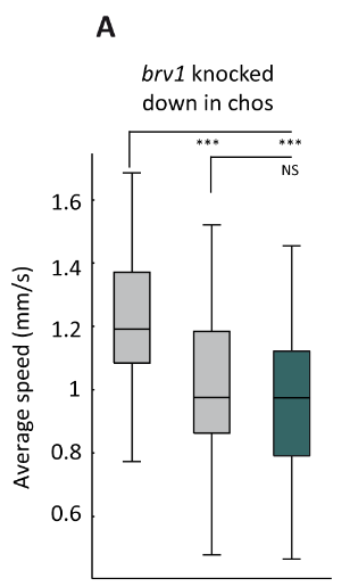

B
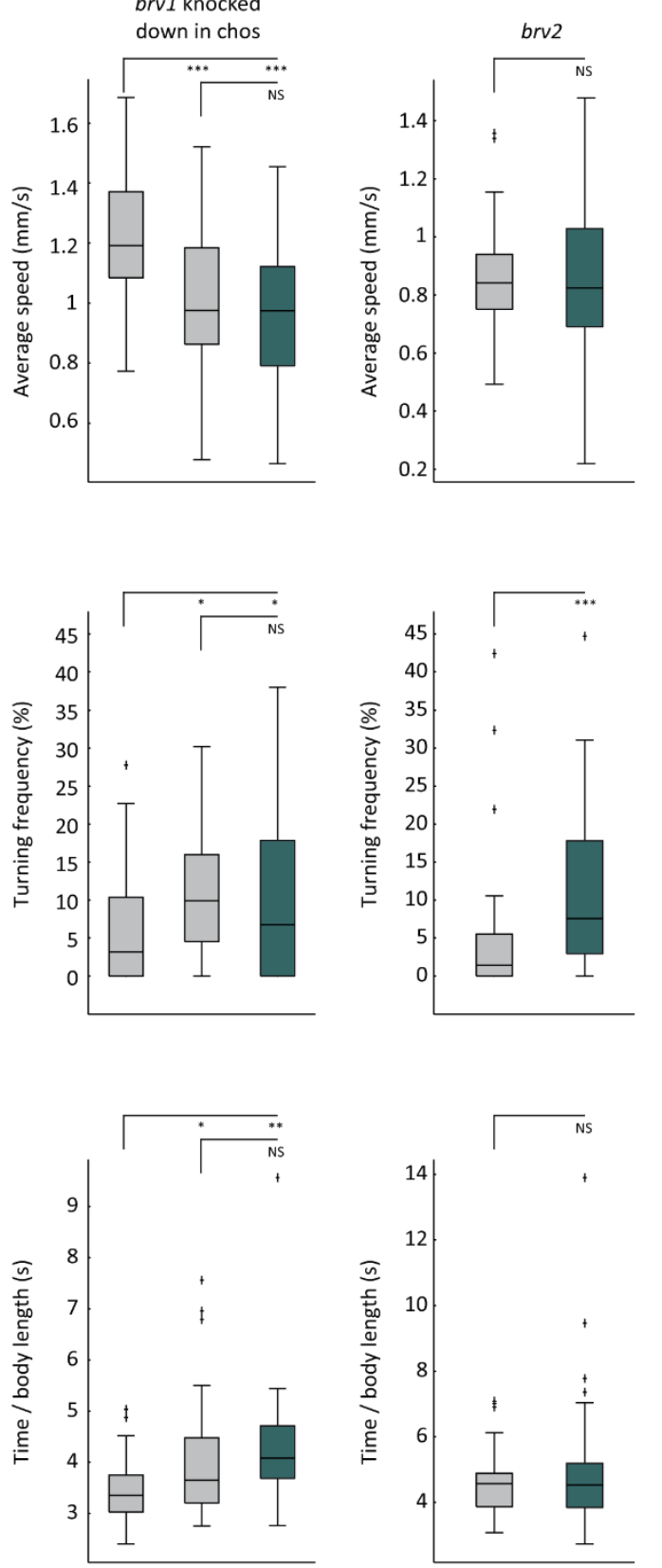

C
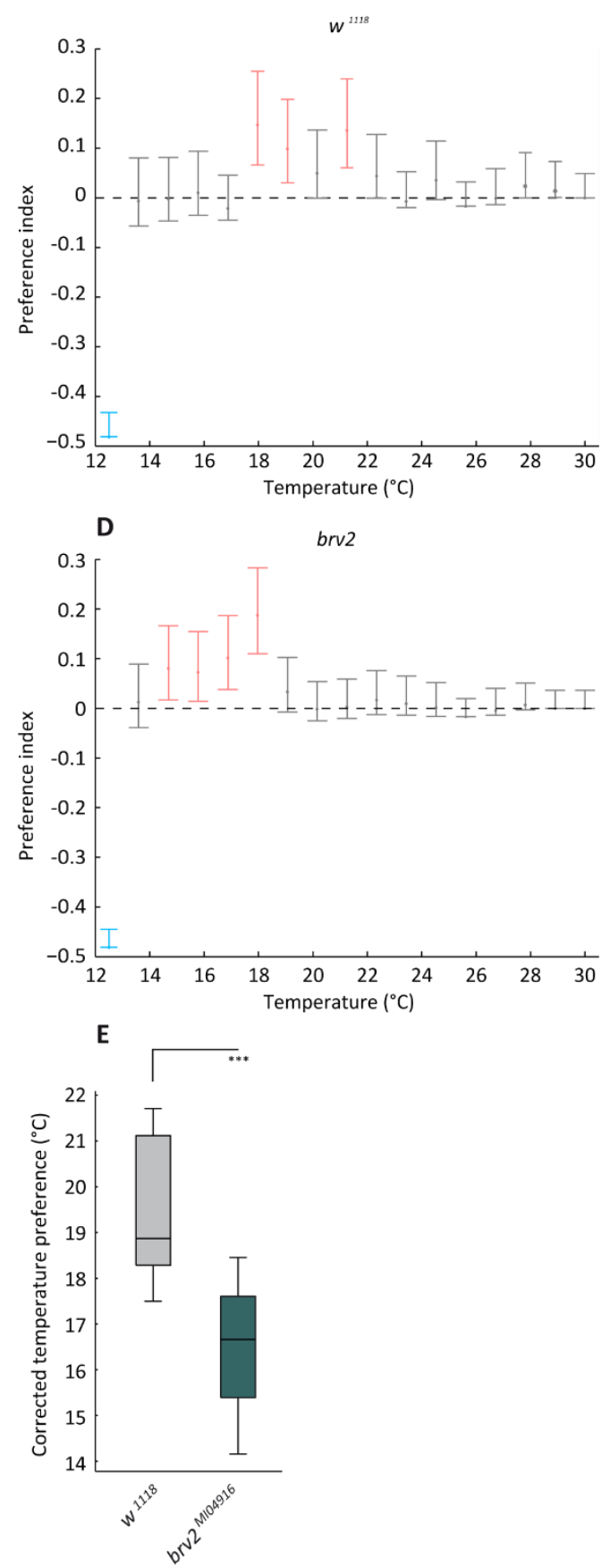
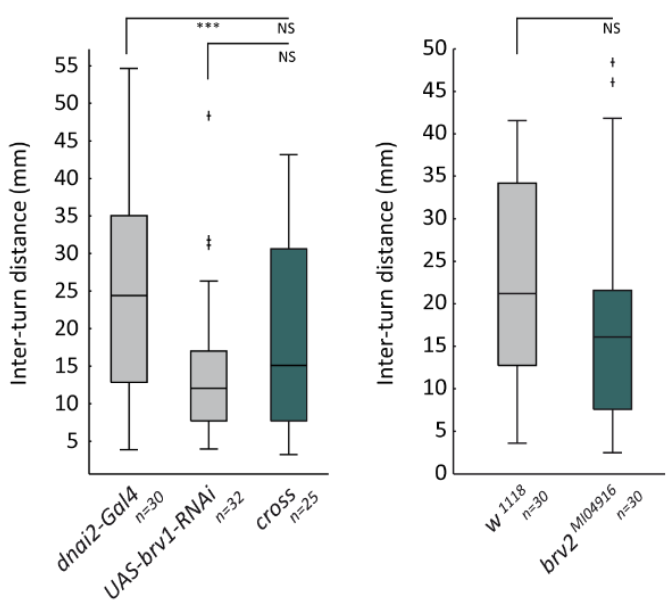


\section{Chapter 3 - Results}

Figure 39. Locomotor performance of cho specific brv1 knockdown and brv2 mutants, and temperature preferences of brv2 mutants. A) Quantification of locomotion behaviour of larvae with a knockdown of brv1 in cho neurons by driving RNAi for brv1 in chos using the cho specific driver dnai2-Gal4. B) Quantification of locomotion behaviour of brv2 $2^{\text {MIO4916 }}$ mutants. C-D) Cold trapped corrected distribution of larvae in a temperature gradient. After the correction (see chapter 1), a preference index is obtained for each temperature. A negative preference index (blue) means the temperature is avoided and a positive index (red) means the temperature is preferred. The points represent the median and the bars represent the $95 \%$ confidence interval. If the $95 \%$ confidence interval overlaps with zero (grey) the temperature is considered as neither preferred nor avoided. C) Gradient distribution of $w^{1118}$. D) Gradient distribution of brv2 ${ }^{\text {M104916 }}$ mutants. E) Temperature preferences of the corrected distributions in C and D. The data in A, B and E are represented as in Figure 37. Significant differences from parental lines and wild-type controls (Fisher's exact permutation tests with Benjamini - Hochberg correction): ${ }^{*}=p<0.05,{ }^{* *}=p<0.01,{ }^{* * *}=p<0.001$, NS = not significant. A-B) n: dnai2Gal4 $=30$, UAS-brv1-RNAi $=32$, cross $=25, w^{1118}=30, b r v 2^{\text {MI04916 }}=30$. C-E) $\mathrm{n}: w^{1118}=74$, brv2 $2^{\text {M104916 }}=100$. 


\subsection{Discussion}

\subsubsection{Lch5 and thermosensation}

Larval chos have been implicated in warm sensation and cold sensation (Kwon et al., 2010; Liu et al., 2003). However, since the first report of a larval cho thermosensitive role (Liu et al., 2003), only one additional study has focused on this issue, favouring the hypothesis that larval chos are involved in cold sensation but not warm sensation (Kwon et al., 2010). This study only focused on behaviour and provided no physiological data to support the cold thermosensory idea. The first study did report physiological responses of Ich5 neurons to thermosensation, but the changes of temperatures used were very high and the responses observed were rather low (Liu et al., 2003).

To date, no molecule involved in thermosensation has been reported in chos. Inactive, a channel involved in mechanosensation, was reported to be necessary for cold sensation, but it was not activated by cold temperatures when expressed ectopically, suggesting it is not a thermosensitive channel. In the present study, it is shown that expression of Brivido1, a channel that has been implicated in cold sensation in adults, is found in two neurons of the larval Ich5. No expression of the IR25a, which has been implicated in larval cold sensation, or the warm sensitive channel GR28B(D) was found.

To determine the thermosensitive role of Ich5 neurons, CaMPARI was used to determine if Ich5 neurons were active during warm or cold stimulation. Lch5 neurons were not more active during cold or warm stimulation, indicating that Ich5 neurons do not respond to temperature stimuli. Although Brivido1 expression suggests only chos 1 and 3 of the Ich5 may be thermosensitive, these neurons did not seem to be more active when stimulated with cold or warm temperatures. Some activity was found in all neurons, but this activity most likely comes from the spontaneous activity of and the proprioceptive role of Ich5 neurons (Zanini, Giraldo, et al., 2018). Lch5 neurons of larvae that were not exposed to UV-light did not show any CaMPARI photoconversion, suggesting that the photoconversion observed in stimulated larvae comes from Ich5 neuron activity and that CaMPARI is sensitive enough to detect the activity of these neurons. Calcium imaging experiments could be carried out to assess Ich 5 responses to temperature changes in a fillet preparation. However, when these experiments were carried out, the changes in temperature generate too much movement of the neurons, which did not allow to keep them in focus and assess the actual changes in fluorescence. 
The behavioural study that implicated chos in cold thermosensation did not specify whether it was all larval chos, or only a subset of these that played a thermosensitive role (Kwon et al., 2010). Kwon et al. in 2010 silenced cho neurons which lead to cold avoidance deficits. Nonetheless, all larval chos were targeted, and whether it is all chos or only a subset of them that mediate cold avoidance was not assessed. In the present study, the responses to temperature of Ich1 and the two ventral chos (i.e., vchA and vchB) were also not evaluated. It is possible that the Ich1 or the ventral chos are the organs mediating thermosensation, and Ich5 only mediate mechanosensation. Although the responses of Ich5 neurons to mechanical stimuli has been studied at a physiological level (Scholz et al., 2015, 2017; Zanini, Giraldo et al., 2018; Zhang et al., 2013), a characterisation of the other larval chos has yet to be made, and whether they are only mechanosensory or mediate other senses is still unclear.

\subsubsection{Brivido1 expression in larval body wall neurons}

brv1 was found to be expressed in different kinds of larval body wall neurons. Interestingly, expression was only found in two of the five neurons of the Ich5. Other channels expressed in larval chos (i.e., Inactive, Nanchung, and NOMPC) are always expressed in all five units, making this pattern of expression of Brivido1 unique. Whether this implies a different function between a subgroup of Ich5 neurons is not clear. All five neurons seemed to be unresponsive to temperature stimuli, and CaMPARI photoconversion reflected a similar activity of all. If they show different responses to various mechanical stimuli (i.e. stretch, touch, or vibrations) remains to be analysed. The localisation of channels in Ich5 neurons is also heterogeneous since NOMPC is localised in a wider range of the cilium in neurons 2 and 4 (Zanini, Giraldo, et al., 2018). This difference in channel localisation and expression may reflect different modalities of Ich5 neurons. Immunostainings of Brivido1 may help clarify whether expression is in fact restricted to neurons 1 and 3 as found with brv1-Gal4 expression.

Brivido1 expression was previously found in adult chos in the Johnston's organ, but if the expression is also restricted to a subset of neurons was not assessed (Gallio et al., 2011). The mechanism of Brivido1 function in chos remains unclear. In the current study, no deficits in proprioception or thermosensation were found for larvae with a brv1 knockdown in chos. Brivido1 cannot be activated by cold temperatures, and even though it is essential for cold sensation in adults, the mechanisms by which it mediates thermosensation has not been examined. One possibility is that Brivido1 may be interacting with an unknown cold sensitive channel, or regulating its activity (Fowler and Montell, 2013). The absence of such channel in larval chos would explain why no thermosensitive deficits were observed in brv1 cho knockdown larvae. Another possibility 
is that the knockdown was not efficient enough to see a thermosensitive phenotype. However, the lack of responses of Ich5 neurons to cold temperatures supports the idea that Brivido1 does not play a thermosensory role in chos.

An ineffective knockdown could also mean that a proprioceptive role or modulation of the activity of channels involved in mechanosensation cannot be excluded. Calcium imaging to mechanical responses (see Chapter 2) in brv1 mutants could help characterise such role. The data obtained for brv2 ${ }^{\text {M104916 }}$ suggests that Brivido2 does not play a role in proprioception, but it does in thermosensation. If as found for adults, Brivido1 and Brivido2 are coexpressed in the same cells and serve a redundant function in larvae, it would mean the Brivido1 only plays a thermosensory role in non-cho neurons, and not a proprioceptive one. However, a function in touch or vibration sensation, also mediated by chos is still possible. Unfortunately, the unavailability of a brv1 mutant did not make it possible to study the proprioceptive role of Brivido1 in this study.

In addition to the cold cells in the arista and Johnston's organ of the adult fly (Gallio et al., 2011), Brivido1 is now found to be expressed in two neurons of the larval Ich5, IdaB, ddaF and dbd multidendritic neurons, and neuromuscular junction. These neurons have been implicated in thermosensation (i.e., cold cells and dbd) (Gallio et al., 2011; Shen et al., 2011), and touch, hearing, or proprioception (i.e., Johnston's organ, Ich5, IdaB and ddaF) (Caldwell et al., 2003; Kernan, 2007; Tsubouchi et al., 2012; Yan et al., 2013; Zanini, Giraldo et al., 2018). Although ion channels may play an important role in development (Georgiev et al., 2010; Montell, 2005), a developmental role of Brivido1 is unlikely since expression was found the two of the three main types of sensory neurons ( $m d$ and cho), but only a subset in each group.

Brivido channels have sequence homology with PKD1 proteins present in mammals (Fowler and Montell, 2013; Gallio et al., 2011). These proteins bind to TRPP2 channels via their C-termini and from a functional calcium channel (Hanaoka et al., 2000; Tsiokas et al., 1997). The channel resulting from PKD1 and TRPP2 interaction is believed to be mechanosensory, and is essential for renal function in humans (Nauli et al., 2003; Tsiokas, 2009). In addition to Brivido1, the Johnston's organ, Ich5, ddaF and dbd neurons express the mechanosensory channel NOMPC (Cheng et al., 2010; Walker et al., 2000; Yan et al., 2013; Zhang et al., 2013). A similar interaction between NOMPC and Brivido1 may be happening in neurons where these proteins are coexpressed. An interaction of these proteins in only two of the Ich5 neurons may explain the different mechanosensory modalities found for larval chos (i.e., proprioception, gentle touch, and vibration), suggesting that the different neurons in the Ich5 may have different mechanosensory roles. ddaF neurons also mediate gentle touch in larvae (Yan et al., 2013). It is possible that Brivido1 interacts with NOMPC 
in these neurons and Ich5 neurons to increase its sensitivity, allowing for the sensation of low intensity mechanical stimuli. Mechanosensory deficits in larvae and adults of brv1 mutants should be assessed to discover a possible mechanosensory role and interaction of Brivido1 and NOMPC.

Brivido1 expression in the neuromuscular junction suggests a role beyond sensory systems. Inactive, which is a channel involved in mechanosensation in chos, is also expressed in the larval neuromuscular junction and it mediates resting $\mathrm{Ca}^{2+}$ concentrations in the presynapse (Wong et al., 2014). It is possible that Brivido1 interacts in chos or the neuromuscular junction with Inactive.

\subsection{Conclusions}

Larval chos have been implicated in thermosensation. In this study, however, no response of Ich5 neurons to warm or cool stimuli was found using the calcium sensor CaMPARI. The role of other larval chos in thermosensation was not assaessed, leaving open the possibility that it is these chos and not Ich5 neurons that mediate larval thermosensation. Brivido1, which is implicated in thermosensation in adults was found to be expressed in two larval Ich5 neurons. However, a cho knockdown of brv1 did not result in an impairment in larval thermosensation. In addition, no proprioceptive phenotype was observed in these larvae. It is still possible that Brivido1 is implicated in touch or vibration sensation in larvae, which are also mediated by larval chos. This is the first time that expression of an ion channel is found in only a subset of Ich5 neurons, which may reflect different roles of the five neurons of the Ich5. Brivido was also expressed in $\mathrm{dbd}$, ddaF and IdaB neurons in the body wall. As Ich5, dbd and ddaF neurons also express NOMPC, a channel that has been implicated in mechanosensation. Future studies should focus on an interaction between these two proteins, and if NOMPC activity is modulated by Brivido1. The presence of Brivido1 in different neurons that serve different sensory roles provides further evidence for the protosensory cell theory. Different sensory modalities share common molecules, which suggests that these molecules served an ancestral function in the protosensory cell and were later adapted in different sensory modalities through evolution. 


\section{General conclusions}

Evidence that different sensory systems have a common origin has accumulated in the recent years. The roles of different genes in mechanosensation and thermosensation were studied. To study thermosensation in temperature gradients, a null model (IGLOO) was generated to correct for cold trapping biases resulting from the temperature dependence of locomotion of insects. This model successfully corrected this bias, which allowed to obtain more accurate temperature preferences of Drosophila larvae and adults, and allowed to extract different features of larval temperature gradient behaviour.

In the present study, additional evidence supporting the theory of the common evolutionary origin of senses was provided. Rhodopsins were found to not only be involved in light sensation and thermosensation, but also in mechanosensation. Larvae of Drosophila were found to require Rhodopsins in their chordotonal organs for locomotion. Deficits in locomotion came from lack of proprioceptive feedback during larval crawling provided by these organs. The data obtained showed that the maintenance of dendritic cilia of these chordotonal neurons is dependent on Rhodopsins. Despite these opsins being rhabdomeric in nature, they are now found to serve structural roles in ciliary cells. Since rhabdomere structure is also dependent on Rhodopsins, the evidence found suggests an ancestral structural role of Rhodopsins, previous to their photosensory roles in photoreceptors.

Brivido1, a molecule involved in Drosophila adult thermosensation, was found to be expressed in mechanosensory and thermosensory neurons. No thermosensory function or proprioceptive functions were found for Brivido1 in larval chordotonal neurons. However, coexpression with the mechanosensory channel NOMPC in different neuronal types suggests a possible interaction between Brivido1, and a modulatory of mechanosensation by this protein. The structural role of Rhodopsins in mechanosensors and the presence of Brivido1 in different sensory neurons provides further evidence to support the hypothesis that sensory systems evolved from an ancestral protosensory cell. The evidence suggests that molecules present in an ancestral sensory cell were adapted to serve new roles in different senses throughout evolution. 


\section{References}

Adrian, G.J., Czarnoleski, M., and Angilletta, M.J. (2016). Flies evolved small bodies and cells at high or fluctuating temperatures. Ecol. Evol. 6, 7991-7996.

Ahmad, S.T., Natochin, M., Artemyev, N.O., and O'Tousa, J.E. (2007). The Drosophila rhodopsin cytoplasmic tail domain is required for maintenance of rhabdomere structure. FASEB J. 21, 449455.

Ainsley, J.A., Pettus, J.M., Bosenko, D., Gerstein, C.E., Zinkevich, N., Anderson, M.G., Adams, C.M., Welsh, M.J., and Johnson, W.A. (2003). Enhanced locomotion caused by loss of the Drosophila DEG/ENaC protein pickpocket1. Curr. Biol. 13, 1557-1563.

Akitake, B., Ren, Q., Boiko, N., Ni, J., Sokabe, T., Stockand, J.D., Eaton, B. a., and Montell, C. (2015). Coordination and fine motor control depend on Drosophila TRP $\gamma$. Nat. Commun. 6, 7288.

Anderson, J.L., Albergotti, L., Proulx, S., Peden, C., Huey, R.B., and Phillips, P.C. (2007). Thermal preference of Caenorhabditis elegans: a null model and empirical tests. J. Exp. Biol. 210, 3107-3116.

Barbagallo, B., and Garrity, P.A. (2015). Temperature sensation in Drosophila. Curr. Opin. Neurobiol. 34, 8-13.

Basca, C.A., Talos, M., and Brad, R. (2005). Randomized Hough Transform for Ellipse Detection with Result Clustering. Proc. Int. Conf. Comput. as a Tool EUROCON 2, 1397-1400.

Benzer, S. (1967). Behavioral mutants of Drosophila isolated by countercurrent distribution. Proc. Natl. Acad. Sci. 58, 1112.

Berrigan, D., and Partridge, L. (1997). Influence of temperature and activity on the metabolic rate of adult Drosophila melanogaster. Comp. Biochem. Physiol. Part A Physiol. 118, 1301-1307.

Birnbaumer, L. (2009). The TRPC class of ion channels: a critical review of their roles in slow, sustained increases in intracellular $\mathrm{Ca}^{2+}$ concentrations. Annu. Rev. Pharmacol. Toxicol. 49, 395426.

Blackburn, T.M., Gaston, K.J., and Loder, N. (1999). Geographic gradients in body size: A clarification of Bergmann's rule. Divers. Distrib. 5, 165-174.

Bloomquist, B.T., Shortridge, R.D., Schneuwly, S., Perdew, M., Montell, C., Steller, H., Rubin, G., and Pak, W.L. (1988). Isolation of a Putative Phospholipase C Gene of Drosophila, norpA, and its Role in Phototransduction. Cell 54, 723-733.

Bodmer, R., and Jan, Y.N. (1987). Morphological differentiation of the embryonic peripheral neurons in Drosophila. Rouxs Arch. Dev. Biol. 196, 69-77.

Brand, a H., and Perrimon, N. (1993). Targeted gene expression as a means of altering cell fates and generating dominant phenotypes. Development 118, 401-415.

Brewster, R., and Bodmer, R. (1995). Origin and specification of type II sensory neurons in Drosophila. Development 121, 2923-2936. 
Burton, V., Mitchell, H.K., Young, P., and Petersen, N.S. (1988). Heat shock protection against cold stress of Drosophila melanogaster. Mol. Cell. Biol. 8, 3550-3552.

Caldwell, J.C., Miller, M.M., Wing, S., Soll, D.R., and Eberl, D.F. (2003). Dynamic analysis of larval locomotion in Drosophila chordotonal organ mutants. Proc. Natl. Acad. Sci. U. S. A. 100, 1605316058.

Chen, C., Buhl, E., Xu, M., Croset, V., Rees, J.S., Lilley, K.S., Benton, R., Hodge, J.J.L., and Stanewsky, R. (2015). Drosophila lonotropic Receptor 25a mediates circadian clock resetting by temperature. Nature 527, 516-520.

Chen, T.W., Wardill, T.J., Sun, Y., Pulver, S.R., Renninger, S.L., Baohan, A., Schreiter, E.R., Kerr, R.A., Orger, M.B., Jayaraman, V., et al. (2013). Ultrasensitive fluorescent proteins for imaging neuronal activity. Nature 499, 295-300.

Cheng, L.E., Song, W., Looger, L.L., Jan, L.Y., and Jan, Y.N. (2010). The role of the TRP channel NompC in Drosophila larval and adult locomotion. Neuron 67, 373-380.

Chou, W.H., Hall, K.J., Wilson, D.B., Wideman, C.L., Townson, S.M., Chadwell, L. V, and Britt, S.G. (1996). Identification of a novel Drosophila opsin reveals specific patterning of the R7 and R8 photoreceptor cells. Neuron 17, 1101-1115.

Collinson, J.M., Quinn, J.C., Hill, R.E., and West, J.D. (2003). The roles of Pax6 in the cornea, retina, and olfactory epithelium of the developing mouse embryo. Dev. Biol. 255, 303-312.

D'Ausilio, A. (2012). Arduino: A low-cost multipurpose lab equipment. Behav. Res. Methods 44, 305-313.

Denis, V., and Cyert, M.S. (2002). Internal $\mathrm{Ca}^{2+}$ release in yeast is triggered by hypertonic shock and mediated by a TRP channel homologue. J. Cell Biol. 156, 29-34.

Dettman, R.W., Turner, F.R., Hoyle, H.D., and Raff, E.C. (2001). Embryonic expression of the divergent Drosophila $\beta 3$-tubulin isoform is required for larval behavior. Genetics 158, 253-263.

Dhaka, A., Viswanath, V., and Patapoutian, A. (2006). Trp ion channels and temperature sensation. Annu. Rev. Neurosci. 29, 135-161.

Dickinson, M.H. (2000). How Animals Move: An Integrative View. Science. 288, 100-106.

Dijkstra, E.W. (1959). A note on two problems in connexion with graphs. Numer. Math. 1, 269-271.

Dillon, M.E., Wang, G., Garrity, P.A., and Huey, R.B. (2009). Thermal preference in Drosophila. J. Therm. Biol. 34, 109-119.

Dillon, M.E., Liu, R., Wang, G., and Huey, R.B. (2012). Disentangling thermal preference and the thermal dependence of movement in ectotherms. J. Therm. Biol. 37, 631-639.

Duffy, J.B. (2002). GAL4 system in Drosophila: A fly geneticist's swiss army knife. Genesis 34, 1-15.

Eberl, D.F. (1999). Feeling the vibes: chordotonal mechanisms in insect hearing. Curr. Opin. Neurobiol. 9, 389-393. 


\section{References}

Edelstein, A.D., Tsuchida, M.A., Amodaj, N., Pinkard, H., Vale, R.D., and Stuurman, N. (2014). Advanced methods of microscope control using $\mu$ Manager software. J. Biol. Methods 1, 10.

Effertz, T., Wiek, R., and Göpfert, M.C. (2011). NompC TRP channel is essential for Drosophila sound receptor function. Curr. Biol. 21, 592-597.

Feuda, R., Hamilton, S.C., Mclnerney, J.O., and Pisani, D. (2012). Metazoan opsin evolution reveals a simple route to animal vision. Proc. Natl. Acad. Sci. 107, 7097-7097.

Field, L.H., and Matheson, T. (1998). Chordotonal Organs of Insects. Adv. In Insect Phys. 27.

Fire, A., Xu, S., Montgomery, M.K., Kostas, S.A., Driver, S.E., and Mello, C.C. (1998). Potent and specific genetic interference by double-stranded RNA in Caenorhabditis elegans. Nature 391, 806811.

Fosque, B.F., Sun, Y., Dana, H., Yang, C.-T., Ohyama, T., Tadross, M.R., Patel, R., Zlatic, M., Kim, D.S., Ahrens, M.B., et al. (2015). Labeling of active neural circuits in vivo with designed calcium integrators. Science. 347, 755-760.

Fowler, M.A., and Montell, C. (2013). Drosophila TRP channels and animal behavior. Life Sci. 92, 394-403.

Fritzsch, B., Piatigorsky, J., Tessmar-Raible, K., Jékely, G., Guy, K., Raible, F., Wittbrodt, J., and Arendt, D. (2005). Ancestry of Photic and Mechanic Sensation? Science. 308, 1113-1114.

Gallio, M., Ofstad, T.A., Macpherson, L.J., Wang, J.W., and Zuker, C.S. (2011). The Coding of Temperature in the Drosophila Brain. Cell 144, 614-624.

Gao, Z., Ruden, D.M., and Lu, X. (2003). PKD2 Cation Channel Is Required for Directional Sperm Movement and Male Fertility. Curr. Biol. 13, 2175-2178.

Gargano, J.W., Martin, I., Bhandari, P., and Grotewiel, M.S. (2005). Rapid iterative negative geotaxis (RING): a new method for assessing age-related locomotor decline in Drosophila. Exp. Gerontol. 40, 386-395.

Garrity, P.A., Goodman, M.B., Samuel, A.D., and Sengupta, P. (2010). Running hot and cold: Behavioral strategies, neural circuits, and the molecular machinery for thermotaxis in $C$. elegans and Drosophila. Genes Dev. 24, 2365-2382.

Georgiev, P., Okkenhaug, H., Drews, A., Wright, D., Lambert, S., Flick, M., Carta, V., Martel, C., Oberwinkler, J., and Raghu, P. (2010). TRPM channels mediate zinc homeostasis and cellular growth during Drosophila larval development. Cell Metab. 12, 386-397.

Ghysen, A. (2013). Origin and evolution of the nervous system. Int. J. Dev. Biol. 47, 555-562.

Ghysen, A., Dambly-chaudire, C., Aeeves, E., Jan, L., and Jan, Y. (1986). Sensory neurons and peripheral pathways in Drosophila embryos. Roux's Arch Dev Biol 195, 222-228.

Gibert, P., and Huey, R.B. (2001). Chill-Coma Temperature in Drosophila: Effects of Developmental Temperature, Latitude, and Phylogeny. Physiol. Biochem. Zool. 74, 429-434. 
Gibert, P., Huey, R.B., and Gilchrist, G.W. (2001). Locomotor performance of Drosophila melanogaster: interactions among developmental and adult temperatures, age, and geography. Evolution (N. Y). 55, 205-209.

Gong, Z., Son, W., Chung, Y.D., Kim, J., Shin, D.W., McClung, C. a, Lee, Y., Lee, H.W., Chang, D.-J., Kaang, B.-K., et al. (2004). Two interdependent TRPV channel subunits, inactive and Nanchung, mediate hearing in Drosophila. J. Neurosci. 24, 9059-9066.

Göpfert, M.C., and Henning, M. (2016). Hearing in Insects. Annu. Rev. Entoomology 15, 257-276.

Green, P., Hartenstein, A.Y., and Hartenstein, V. (1993). The embryonic development of the Drosophila visual system. Cell Tissue Res. 273, 583-598.

Grether, M.E., Abrams, J.M., Agapite, J., White, K., and Steller, H. (1995). The head involution defective gene of Drosophila melanogaster functions in programmed cell death. Genes Dev. 9, 1694-1708.

Grueber, W.B., Jan, L.Y., and Jan, Y.N. (2002). Tiling of the Drosophila epidermis by multidendritic sensory neurons. Development 129, 2867-2878.

Günther, M.N., Nettesheim, G., and Shubeita, G.T. (2016). Quantifying and predicting Drosophila larvae crawling phenotypes. Sci. Rep. 6, 27972.

Hamada, F.N., Rosenzweig, M., Kang, K., Pulver, S.R., Ghezzi, A., Jegla, T.J., and Garrity, P.A. (2008). An internal thermal sensor controlling temperature preference in Drosophila. Nature 454, 217-220.

Hanaoka, K., Qian, F., Boletta, A., Bhunia, A.K., Piontek, K., Tsiokas, L., Sukhatme, V.P., Guggino, W.B., and Germino, G.G. (2000). Co-assembly of polycystin-1 and -2 produces unique cationpermeable currents. Nature 408, 990-994.

Hardie, R.C. (2001). Phototransduction in Drosophila melanogaster. J. Exp. Biol. 204, 3403-3409.

Hardie, R.C., and Franze, K. (2012). Photomechanical responses in Drosophila photoreceptors. Science. 338, 260-263.

Harris, W.A., Ready, D.F., Lipson, E.D., and Hudspeth, A.J. (1977). Vitamin A deprivation and Drosophila photopigments. Nature 267, 648-650.

Hartman, B., Lee, P.N., Kang, Y.Y., Tomarev, S., de Couet, H.G., and Callaerts, P. (2003). Pax6 in the sepiolid squid Euprymna scolopes: evidence for a role in eye, sensory organ and brain development. Mech. Dev. 120, 177-183.

Heckscher, E.S., Lockery, S.R., and Doe, C.Q. (2012). Characterization of Drosophila larval crawling at the level of organism, segment, and somatic body wall musculature. J. Neurosci. 32, 1246012471.

Hofmann, T., Chubanov, V., Chen, X., Dietz, A.S., Gudermann, T., and Montell, C. (2010). Drosophila TRPM channel is essential for the control of extracellular magnesium levels. PLoS One 5, e10519.

Hori, Y., and Kimura, M.T. (1998). Relationship between Cold Stupor and Cold Tolerance in Drosophila (Diptera: Drosophilidae). Environ. Entomol. 27, 1297-1302. 
Howard, J., and Bechstedt, S. (2004). Hypothesis: A helix of ankyrin repeats of the NOMPC-TRP ion channel is the gating spring of mechanoreceptors. Curr. Biol. 14, 224-226.

Huber, A., Schulz, S., Bentrop, J., Groell, C., Wolfrum, U., and Paulsen, R. (1997). Molecular cloning of Drosophila Rh6 rhodopsin: The visual pigment of a subset of R8 photoreceptor cells. FEBS Lett. 406, 6-10.

Hughes, C.L., and Thomas, J.B. (2007). A sensory feedback circuit coordinates muscle activity in Drosophila. Mol. Cell. Neurosci. 35, 383-396.

Hwang, R.Y., Zhong, L., Xu, Y., Johnson, T., Zhang, F., Deisseroth, K., and Tracey, W.D. (2007). Nociceptive Neurons Protect Drosophila Larvae from Parasitoid Wasps. Curr. Biol. 17, 2105-2116.

Jalal, M., Andersen, T., and Hessen, D.O. (2015). Temperature and developmental responses of body and cell size in Drosophila; effects of polyploidy and genome configuration. J. Therm. Biol. 51, 1-14.

Jarman, A.P. (2002). Studies of mechanosensation using the fly. Hum. Mol. Genet. 11, 1215-1218.

Jarman, A.P., Sun, Y., Jan, L.Y., and Jan, Y.N. (1995). Role of the proneural gene, atonal, in formation of Drosophila chordotonal organs and photoreceptors. Development 121, 2019-2030.

Jensen, D., Overgaard, J., and Sørensen, J.G. (2007). The influence of developmental stage on cold shock resistance and ability to cold-harden in Drosophila melanogaster. J. Insect Physiol. 53, 179186.

Karak, S., Jacobs, J.S., Kittelmann, M., Spalthoff, C., Katana, R., Sivan-Loukianova, E., Schon, M.A., Kernan, M.J., Eberl, D.F., and Göpfert, M.C. (2015). Diverse Roles of Axonemal Dyneins in Drosophila Auditory Neuron Function and Mechanical Amplification in Hearing. Sci. Rep. 5, 1-12.

Kavlie, R.G., and Albert, J.T. (2013). Chordotonal organs. Curr. Biol. 23, R334-R335.

Kelty, J.D., and Lee Jr, R.E. (2001). Cold-hardening of Drosophila melanogaster (Diptera: Drosophilidae) during ecologically based thermoperiodic cycles. J. Exp. Biol. 204, 1659-1666.

Kernan, M.J. (2007). Mechanotransduction and auditory transduction in Drosophila. Pflugers Arch. $454,703-720$.

Kernan, M., Cowan, D., and Zuker, C. (1994). Genetic Dissection of Mechanosensory Transduction : Mechanoreception-Defective Mutations of Drosophila. Neuron 12, 1195-1206.

Kim, J., Chung, Y.D., Park, D.-Y., Choi, S., Shin, D.W., Soh, H., Lee, H.W., Son, W., Yim, J., Park, C.-S., et al. (2003). A TRPV family ion channel required for hearing in Drosophila. Nature 424, 81-84.

Kim, S.H., Lee, Y., Akitake, B., Woodward, O.M., Guggino, W.B., and Montell, C. (2010). Drosophila TRPA1 channel mediates chemical avoidance in gustatory receptor neurons. Proc. Natl. Acad. Sci. $107,8440-8445$.

Klein, M., Afonso, B., Vonner, A.J., Hernandez-Nunez, L., Berck, M., Tabone, C.J., Kane, E.A., Pieribone, V.A., Nitabach, M.N., Cardona, A., et al. (2015). Sensory determinants of behavioral dynamics in Drosophila thermotaxis. Proc. Natl. Acad. Sci. 112, E220-E229. 
Knecht, Z.A., Silbering, A.F., Ni, L., Klein, M., Budelli, G., Bell, R., Abuin, L., Ferrer, A.J., Samuel, A.D., Benton, R., et al. (2016). Distinct combinations of variant ionotropic glutamate receptors mediate thermosensation and hygrosensation in Drosophila. Elife 5, e17979.

Köttgen, M., Hofherr, A., Li, W., Chu, K., Cook, S., Montell, C., and Watnick, T. (2011). Drosophila sperm swim backwards in the female reproductive tract and are activated via TRPP2 ion channels. PLoS One 6, e20031.

Krebs, R.A. (1999). A comparison of Hsp70 expression and thermotolerance in adults and larvae of three Drosophila species. Cell Stress Chaperones 4, 243-249.

Krstevska, B., and Hoffmann, A.A. (1994). The Effects of Acclimation and Rearing Conditions on the Response of Tropical and Temperate Populations of Drosophila melanogaster and D. simulans to a Temperature Gradient (Diptera: Drosophilidae). J. Insect Behav. 7, 279-288.

Kumar, J.P., and Ready, D.F. (1995). Rhodopsin plays an essential structural role in Drosophila photoreceptor development. Development 121, 4359-4370.

Kwon, J.Y., Dahanukar, A., Weiss, L.A., and Carlson, J.R. (2007). The molecular basis of $\mathrm{CO}_{2}$ reception in Drosophila. Proc. Natl. Acad. Sci. U. S. A. 104, 3574-3578.

Kwon, Y., Shim, H.-S., Wang, X., and Montell, C. (2008). Control of thermotactic behavior via coupling of a TRP channel to a phospholipase C signaling cascade. Nat. Neurosci. 11, 871-873.

Kwon, Y., Shen, W.L., Shim, H.-S., and Montell, C. (2010). Fine thermotactic discrimination between the optimal and slightly cooler temperatures via a TRPV channel in chordotonal neurons. J. Neurosci. 30, 10465-10471.

Lai, S.L., and Lee, T. (2006). Genetic mosaic with dual binary transcriptional systems in Drosophila. Nat. Neurosci. 9, 703-709.

Lee, Y., Lee, Y., Lee, J., Bang, S., Hyun, S., Kang, J., Hong, S.-T., Bae, E., Kaang, B.-K., and Kim, J. (2005). Pyrexia is a new thermal transient receptor potential channel endowing tolerance to high temperatures in Drosophila melanogaster. Nat. Genet. 37, 305-310.

Lehnert, B.P., Baker, A.E., Gaudry, Q., Chiang, A.S., and Wilson, R.I. (2013). Distinct Roles of TRP Channels in Auditory Transduction and Amplification in Drosophila. Neuron 77, 115-128.

Leroi, A.M., Bennett, A.F., and Lenski, R.E. (1994). Temperature acclimation and competitive fitness: an experimental test of the beneficial acclimation assumption. Proc. Natl. Acad. Sci. 91, 1917-1921.

Leung, N.Y., and Montell, C. (2017). Unconventional Roles of Opsins. Annu. Rev. Cell Dev. Biol. 33, 2.1-2.24.

Li, K., and Gong, Z. (2016). Feeling Hot and Cold: Thermal Sensation in Drosophila. Neurosci. Bull.

von Lintig, J., Dreher, A., Kiefer, C., Wernet, M.F., and Vogt, K. (2001). Analysis of the blind Drosophila mutant ninaB identifies the gene encoding the key enzyme for vitamin $A$ formation in vivo. Proc. Natl. Acad. Sci. U. S. A. 98, 1130-1135.

Liu, L., Yermolaieva, O., Johnson, W. a, Abboud, F.M., and Welsh, M.J. (2003). Identification and function of thermosensory neurons in Drosophila larvae. Nat. Neurosci. 6, 267-273. 
Liu, L., Li, Y., Wang, R., Yin, C., Dong, Q., Hing, H., Kim, C., and Welsh, M.J. (2007). Drosophila hygrosensation requires the TRP channels water witch and nanchung. Nature 450, 294-298.

MacMillan, H.A., and Sinclair, B.J. (2011). Mechanisms underlying insect chill-coma. J. Insect Physiol. $57,12-20$.

MacMillan, H.A., Andersen, J.L., Loeschcke, V., and Overgaard, J. (2015a). Sodium distribution predicts the chill tolerance of Drosophila melanogaster raised in different thermal conditions. Am. J. Physiol. - Regul. Integr. Comp. Physiol. 308, R823-R831.

MacMillan, H.A., Ferguson, L. V., Nicolai, A., Donini, A., Staples, J.F., and Sinclair, B.J. (2015b). Parallel ionoregulatory adjustments underlie phenotypic plasticity and evolution of Drosophila cold tolerance. J. Exp. Biol. 218, 423-432.

MacMillan, H.A., Knee, J.M., Dennis, A.B., Udaka, H., Marshall, K.E., Merritt, T.J.S., and Sinclair, B.J. (2016). Cold acclimation wholly reorganizes the Drosophila melanogaster transcriptome and metabolome. Sci. Rep. 6, 1-14.

Masek, P., and Keene, A.C. (2013). Drosophila Fatty Acid Taste Signals through the PLC Pathway in Sugar-Sensing Neurons. PLoS Genet. 9, e1003710.

Mazzoni, E.O., Desplan, C., and Blau, J. (2005). Circadian pacemaker neurons transmit and modulate visual information to control a rapid behavioral response. Neuron 45, 293-300.

McKemy, D.D., Neuhausser, W.M., and Julius, D. (2002). Identification of a cold receptor reveals a general role for TRP channels in thermosensation. Nature 416, 52-58.

McMeniman, C.J., Corfas, R.A., Matthews, B.J., Ritchie, S.A., and Vosshall, L.B. (2014). Multimodal integration of carbon dioxide and other sensory cues drives mosquito attraction to humans. Cell $156,1060-1071$.

Mellanby, K. (1939). Low temperature and insect activity. Proc. R. Soc. B 127, 473-487.

Menon, I., Huber, T., Sanyal, S., Banerjee, S., Barré, P., Canis, S., Warren, J.D., Hwa, J., Sakmar, T.P., and Menon, A.K. (2011). Opsin is a phospholipid flippase. Curr. Biol. 21, 149-153.

Mishra, A., Salari, A., Berigan, B.R., Miguel, K.C., Amirshenava, M., Robinson, A., Zars, B.C., Lin, J.L., Milescu, L.S., Milescu, M., et al. (2018). The Drosophila Gr28bD product is a non-specific cation channel that can be used as a novel thermogenetic tool. Sci. Rep. 8, 1-10.

Montell, C. (2005). The TRP superfamily of cation channels. Sci. STKE 2005, 1-24.

Montell, C. (2009). A Taste of the Drosophila Gustatory Receptors. Curr. Opin. Neurobiol. 19, 345353.

Montell, C. (2012). Drosophila visual transduction. Trends Neurosci. 35, 356-363.

Montell, C. (2013). Gustatory receptors: Not just for good taste. Curr. Biol. 23, R929-R932.

Montell, C., Jones, K., Zuker, C., and Rubin, G. (1987). A second opsin gene expressed in the ultraviolet-sensitive R7 photoreceptor cells of Drosophila melanogaster. J. Neurosci. 7, 1558-1566. 
Nadrowski, B., Effertz, T., Senthilan, P.R., and Göpfert, M.C. (2011). Antennal hearing in insects New findings, new questions. Hear. Res. 273, 7-13.

Nauli, S.M., Alenghat, F.J., Luo, Y., Williams, E., Vassilev, P., Li, X., Elia, A.E.H., Lu, W., Brown, E.M., Quinn, S.J., et al. (2003). Polycystins 1 and 2 mediate mechanosensation in the primary cilium of kidney cells. Nat. Genet. 33, 129-137.

Neely, G.G., Keene, A.C., Duchek, P., Chang, E.C., Wang, Q.P., Aksoy, Y.A., Rosenzweig, M., Costigan, M., Woolf, C.J., Garrity, P.A., et al. (2011). TrpA1 regulates thermal nociception in Drosophila. PLoS One 6.

Nesterov, A., Spalthoff, C., Kandasamy, R., Katana, R., Rankl, N.B., Andrés, M., Jähde, P., Dorsch, J.A., Stam, L.F., Braun, F.J., et al. (2015). TRP Channels in Insect Stretch Receptors as Insecticide Targets. Neuron 86, 665-672.

Ni, J.D., Baik, L.S., Holmes, T.C., and Montell, C. (2017). A rhodopsin in the brain functions in circadian photoentrainment in Drosophila. Nature 545, 340-344.

Ni, L., Bronk, P., Chang, E.C., Lowell, A.M., Flam, J.O., Panzano, V.C., Theobald, D.L., Griffith, L.C., and Garrity, P. a (2013). A gustatory receptor paralogue controls rapid warmth avoidance in Drosophila. Nature 500, 580-584.

Ni, L., Klein, M., Svec, K. V., Budelli, G., Chang, E.C., Ferrer, A.J., Benton, R., Samuel, A.D.T., and Garrity, P.A. (2016). The ionotropic receptors IR21a and IR25a mediate cool sensing in Drosophila. eLife 5, 1-12.

Niemeyer, B.A., Suzuki, E., Scott, K., Jalink, K., and Zuker, C.S. (1996). The Drosophila light-activated conductance is composed of the two channels TRP and TRPL. Cell 85, 651-659.

Niwa, N., Hiromi, Y., and Okabe, M. (2004). A conserved developmental program for sensory organ formation in Drosophila melanogaster. Nat. Genet. 36, 293-297.

O'Tousa, J.E., Baehr, W., Martin, R.L., Hirsh, J., Pak, W.L., and Applebury, M.L. (1985). The Drosophila ninaE gene encodes an opsin. Cell 40, 839-850.

Orgogozo, V., and Grueber, W.B. (2005). FlyPNS, a database of the Drosophila embryonic and larval peripheral nervous system. BMC Dev. Biol. 5.

Ozaki, K., Nagatani, H., Ozaki, M., and Tokunaga, F. (1993). Maturation of Major Drosophila Rhodopsin, ninaE, Requires Chromophore 3-Hydroxyretinal. Neuron 10, 1113-1119.

Pak, W.L., Shino, S., and Leung, H.-T. (2012). PDA (Prolonged Depolarizing Afterpotential)-Defective Mutants: The Story of nina's and ina's-pinta and santa maria , Too. J. Neurogenet. 26, 216-237.

Palmer, C.P., Zhou, X.-L., Lin, J., Loukin, S.H., Kung, C., and Saimi, Y. (2001). A TRP homolog in Saccharomyces cerevisiae forms an intracellular $\mathrm{Ca}^{2+}$-permeable channel in the yeast vacuolar membrane. Proc. Natl. Acad. Sci. 98, 7801-7805.

Papatsenko, D., Sheng, G., and Desplan, C. (1997). A new rhodopsin in R8 photoreceptors of Drosophila: evidence for coordinate expression with Rh3 in R7 cells. Development 124, 1665-1673.

Pearson, K.G. (1995). Proprioceptive regulation of locomotion. Curr. Opin. Neurobiol. 5, 786-791. 
Perrimon, N., Ni, J., and Perkins, L. (2010). In vivo RNAi : Today and Tomorrow. Cold Spring Harb. Perspect. Biol. 2, 1-11.

Pollock, J. a, and Benzer, S. (1988). Transcript localization of four opsin genes in the three visual organs of Drosophila; $\mathrm{RH} 2$ is ocellus specific. Nature 333, 779-782.

Pollock, V.P., Radford, J.C., Pyne, S., Hasan, G., Dow, J.A.T., and Davies, S.-A. (2003). norpA and itpr mutants reveal roles for phospholipase $\mathrm{C}$ and inositol $(1,4,5)$ - trisphosphate receptor in Drosophila melanogaster renal function. J. Exp. Biol. 206, 901-911.

Prahlad, A., Spalthoff, C., Kong, D., Großhans, J., Göpfert, M.C., and Schmidt, C.F. (2017). Mechanical Properties of a Drosophila Larval Chordotonal Organ. Biophys. J. 113, 2796-2804.

Python, F., and Stocker, R.F. (2002). Adult-like complexity of the larval antennal lobe of $D$. melanogaster despite markedly low numbers of odorant receptor neurons. J. Comp. Neurol. 445, 374-387.

Qin, W., Neal, S.J., Robertson, R.M., Westwood, J.T., and Walker, V.K. (2005). Cold hardening and transcriptional change in Drosophila melanogaster. Insect Mol. Biol. 14, 607-613.

Ramdya, P., Lichocki, P., Cruchet, S., Frisch, L., Tse, W., Floreano, D., and Benton, R. (2014). Mechanosensory interactions drive collective behaviour in Drosophila. Nature 519, 233-236.

Ramires, M.L. V., Nieto de Castro, C.A., Nagasaka, Y., Nagashima, A., Assael, M.J., and Wakeham, W.A. (1995). Standard Reference Data for the Thermal Conductivity of Water. J. Phys. Chem. Ref. Data 24, 1377-1381.

Ramsey, I.S., Delling, M., and Clapham, D.E. (2006). An introduction to TRP channels. Annu. Rev. Physiol. 68, 619-647.

Ray, C. (1960). The application of Bergmann's and Allen's rules to the poikilotherms. J. Morphol. $106,85-108$.

Riesgo-Escovar, J., Raha, D., and Carlson, J.R. (1995). Requirement for a phospholipase C in odor response: Overlap between olfaction and vision in Drosophila. Proc. Natl. Acad. Sci. U. S. A. 92, 2864-2868.

Risse, B., Thomas, S., Otto, N., Löpmeier, T., Valkov, D., Jiang, X., and Klämbt, C. (2013). FIM, a novel FTIR-based imaging method for high throughput locomotion analysis. PLoS One 8, e53963.

Rosenzweig, M., Brennan, K.M., Tayler, T.D., Phelps, P.O., Patapoutian, A., and Garrity, P. a (2005). The Drosophila ortholog of vertebrate TRPA1 regulates thermotaxis. Genes Dev. 19, 419-424.

Rosenzweig, M., Kang, K., and Garrity, P.A. (2008). Distinct TRP channels are required for warm and cool avoidance in Drosophila melanogaster. Proc. Natl. Acad. Sci. 105, 14668-14673.

Sarfare, S., Ahmad, S.T., Joyce, M. V., Boggess, B., and O'Tousa, J.E. (2005). The Drosophila ninaG oxidoreductase acts in visual pigment chromophore production. J. Biol. Chem. 280, 11895-11901.

Sayeed, O., and Benzer, S. (1996). Behavioral genetics of thermosensation and hygrosensation in Drosophila. Proc. Natl. Acad. Sci. U. S. A. 93, 6079-6084. 
Schindelin, J., Arganda-Carreras, I., Frise, E., Kaynig, V., Longair, M., Pietzsch, T., Preibisch, S., Rueden, C., Saalfeld, S., Schmid, B., et al. (2012). Fiji: An open-source platform for biological-image analysis. Nat. Methods 9, 676-682.

Scholz, N., Gehring, J., Guan, C., Ljaschenko, D., Fischer, R., Lakshmanan, V., Kittel, R.J., and Langenhan, T. (2015). The Adhesion GPCR Latrophilin/CIRL Shapes Mechanosensation. Cell Rep. 11, 866-874.

Scholz, N., Guan, C., Nieberler, M., Grotemeyer, A., Maiellaro, I., Gao, S., Beck, S., Pawlak, M., Sauer, M., Asan, E., et al. (2017). Mechano-dependent signaling by latrophilin/CIRL quenches CAMP in proprioceptive neurons. Elife 6, 1-21.

Scott, K., Becker, A., Sun, Y., Hardy, R., and Zuker, C. (1995). Gq alpha protein function in vivo: genetic dissection of its role in photoreceptor cell physiology. Neuron 15, 919-927.

Sejerkilde, M., Sørensen, J.G., and Loeschcke, V. (2003). Effects of cold- and heat hardening on thermal resistance in Drosophila melanogaster. J. Insect Physiol. 49, 719-726.

Senthilan, P.R., Piepenbrock, D., Ovezmyradov, G., Nadrowski, B., Bechstedt, S., Pauls, S., Winkler, M., Möbius, W., Howard, J., and Göpfert, M.C. (2012). Drosophila Auditory Organ Genes and Genetic Hearing Defects. Cell 150, 1042-1054.

Shanbhag, S.R., Singh, K., and Naresh Singh, R. (1992). Ultrastructure of the femoral chordotonal organs and their novel synaptic organization in the legs of Drosophila melanogaster Meigen (Diptera : Drosophilidae). Int. J. Insect Morphol. Embryol. 21, 311-322.

Shen, W.L., Kwon, Y., Adegbola, A.A., Luo, J., Chess, A., and Montell, C. (2011). Function of Rhodopsin in Temperature Discrimination in Drosophila. Science. 331, 1333-1336.

Shichida, Y., and Matsuyama, T. (2009). Evolution of opsins and phototransduction. Philos. Trans. R. Soc. B 364, 2881-2895.

Shieh, B.-H. (2011). Molecular genetics of retinal degeneration: A Drosophila perspective. Fly. 5, 356-368.

Singh, R.N., and Singh, K. (1984). Fine structure of the sensory organs of Drosophila melanogaster Meigen larva (Diptera : Drosophilidae). Int. J. Insect Morphol. Embryol. 13, 255-273.

Sokabe, T., Tsujiuchi, S., Kadowaki, T., and Tominaga, M. (2008). Drosophila Painless Is a Ca ${ }^{2+}$ Requiring Channel Activated by Noxious Heat. J. Neurosci. 28, 9929-9938.

Sokabe, T., Chen, H.-C., Luo, J., and Montell, C. (2016). A Switch in Thermal Preference in Drosophila Larvae Depends on Multiple Rhodopsins. Cell Rep. 17, 336-344.

Song, W., Onishi, M., Jan, L.Y., and Jan, Y.N. (2007). Peripheral multidendritic sensory neurons are necessary for rhythmic locomotion behavior in Drosophila larvae. Proc. Natl. Acad. Sci. U. S. A. 104, 5199-5204.

Sprecher, S.G., Pichaud, F., and Desplan, C. (2007). Adult and larval photoreceptors use different mechanisms to specify the same Rhodopsin fates. Genes Dev. 21, 2182-2195. 
Stewart, B.A., Atwood, H.L., Renger, J.J., Wang, J., and Wu, C.F. (1994). Improved stability of Drosophila larval neuromuscular preparations in haemolymph-like physiological solutions. J. Comp. Physiol. A 175, 179-191.

Sun, Y., Liu, L., Ben-Shahar, Y., Jacobs, J.S., Eberl, D.F., and Welsh, M.J. (2009). TRPA channels distinguish gravity sensing from hearing in Johnston's organ. Proc. Natl. Acad. Sci. U. S. A. 106, 13606-13611.

Terakita, A. (2005). The opsins. Genome Biol. 6, 213.

Thévenaz, P., Ruttimann, U.E., and Unser, M. (1998). A pyramid approach to subpixel registration based on intensity. IEEE Trans. Image Process. 7, 27-41.

Tracey, W.D., Wilson, R.I., Laurent, G., and Benzer, S. (2003). painless, a Drosophila gene essential for nociception. Cell 113, 261-273.

Tsiokas, L. (2009). Function and regulation of TRPP2 at the plasma membrane. Am. J. Physiol. Physiol. 297, F1-F9.

Tsiokas, L., Kim, E., Arnould, T., Sukhatme, V.P., and Walz, G. (1997). Homo- and heterodimeric interactions between the gene products of PKD1 and PKD2. Proc. Natl. Acad. Sci. 94, 6965-6970.

Tsubouchi, A., Caldwell, J.C., and Tracey, W.D. (2012). Dendritic filopodia, ripped pocket, NOMPC, and NMDARs contribute to the sense of touch in Drosophila larvae. Curr. Biol. 22, 2124-2134.

Turner, H.N., Armengol, K., Patel, A.A., Himmel, N.J., Sullivan, L., Iyer, S.C., Bhattacharya, S., Iyer, E.P.R., Landry, C., Galko, M.J., et al. (2016). The TRP Channels Pkd2, NompC, and Trpm Act in ColdSensing Neurons to Mediate Unique Aversive Behaviors to Noxious Cold in Drosophila. Curr. Biol. 26, 3116-3128.

Venkatachalam, K., and Montell, C. (2007). TRP channels. Annu. Rev. Biochem. 76, 387-417.

Voronoi, G. (1908). Nouvelles applications des paramètres continus à la théorie des formes quadratiques. Deuxième Mémoire. Rech. Sur Les Parallélloèdres Primitifs. J. Reine Angew. Math. $134,198-287$.

Walker, R., Willingham, A., and Zuker, C. (2000). A Drosophila Mechanosensory Transduction Channel. Science. 287, 2229-2234.

Wang, T., and Montell, C. (2007). Phototransduction and retinal degeneration in Drosophila. Pflugers Arch. 454, 821-847.

Wang, J.W., Sylwester, a W., Reed, D., Wu, D. a, Soll, D.R., and Wu, C.F. (1997). Morphometric description of the wandering behavior in Drosophila larvae: aberrant locomotion in $\mathrm{Na}^{+}$and $\mathrm{K}^{+}$ channel mutants revealed by computer-assisted motion analysis. J. Neurogenet. 11, 231-254.

Wang, T., Jiao, Y., and Montell, C. (2007). Dissection of the pathway required for generation of vitamin A and for Drosophila phototransduction. J. Cell Biol. 177, 305-316.

White, K., Grether, M.E., Abrams, J.M., Young, L., Farrell, K., and Steller, H. (1994). Genetic control of programmed cell death in Drosophila. Science. 264, 667-683. 
Wilson, R.I., Turner, G.C., and Laurent, G. (2004). Transformation of Olfactory Representations in the Drosophila Antennal Lobe. Science. 303, 366-370.

Wong, C., Chen, K., Lin, Y.Q., Chao, Y., Duraine, L., Lu, Z., Yoon, W.H., Sullivan, J.M., Broadhead, G.T., Sumner, C.J., et al. (2014). A TRPV Channel in Drosophila Motor Neurons Regulates Presynaptic Resting $\mathrm{Ca}^{2+}$ Levels, Synapse Growth , and Synaptic Transmission. Neuron 84, 764-777.

Wong, C.O., Li, R., Montell, C., and Venkatachalam, K. (2012). Drosophila TRPML is required for TORC1 activation. Curr. Biol. 22, 1616-1621.

Xiang, Y., Yuan, Q., Vogt, N., Looger, L.L., Jan, L.Y., and Jan, Y.N. (2010). Light-avoidance-mediating photoreceptors tile the Drosophila larval body wall. Nature 468, 921-926.

Xie, Y., and Ji, Q. (2002). A new efficient ellipse detection method. Proceedings. 16th Int. Conf. Pattern Recognit. 2, 957-960.

Xu, S.Y., Cang, C.L., Liu, X.F., Peng, Y.Q., Ye, Y.Z., Zhao, Z.Q., and Guo, a K. (2006). Thermal nociception in adult Drosophila: behavioral characterization and the role of the painless gene. Genes. Brain. Behav. 5, 602-613.

Yack, J.E. (2004). The structure and function of auditory chordotonal organs in insects. Microsc. Res. Tech. 63, 315-337.

Yamamoto, A., and Obha, S. (1984). Temperature preferences of eleven Drosophila species from Japan: the relationship between preferred temperature and some ecological characteristics in their natural habitats. Zoolog. Sci. 1, 631-640.

Yan, Z., Zhang, W., He, Y., Gorczyca, D., Xiang, Y., Cheng, L.E., Meltzer, S., Jan, L.Y., and Jan, Y.N. (2013). Drosophila NOMPC is a mechanotransduction channel subunit for gentle-touch sensation. Nature 493, 221-225.

Yang, J., Gao, J., Adamian, M., Wen, X.-H., Pawlyk, B., Zhang, L., Sanderson, M.J., Zuo, J., Makino, C.L., and Li, T. (2005). The Ciliary Rootlet Maintains Long-Term Stability of Sensory Cilia. Mol. Cell. Biol. 25, 4129-4137.

Yau, K.-W., and Hardie, R.C. (2009). Phototransduction motifs and variations. Cell 139, 246-264.

Zanini, D., Giraldo, D., Warren, B., Katana, R., Andrés, M., Reddy, S., Pauls, S., SchwedhelmDomeyer, N., Geurten, B.R.H., and Göpfert, M.C. (2018). Proprioceptive Opsin Functions in Drosophila Larval Locomotion. Neuron 1-8.

Zhang, W., Yan, Z., Jan, L.Y., and Jan, Y.N. (2013). Sound response mediated by the TRP channels NOMPC, NANCHUNG, and INACTIVE in chordotonal organs of Drosophila larvae. Proc. Natl. Acad. Sci. U. S. A. 110, 13612-13617.

Zhu, L., McKay, R.R., and Shortridge, R.D. (1993). Tissue-specific expression of phospholipase C encoded by the norpA gene of Drosophila melanogaster. J. Biol. Chem. 268, 15994-16001.

Zuker, C.S., Cowman, A.F., and Rubin, G.M. (1985). Isolation and structure of a rhodopsin gene from D. melanogaster. Cell 40, 851-858. 


\section{References}

Zuker, C.S., Montell, C., Jones, K., Laverty, T., and Rubin, G.M. (1987). A rhodopsin gene expressed in photoreceptor cell R7 of the Drosophila eye: homologies with other signal-transducing molecules. J. Neurosci. 7, 1550-1557. 


\section{Appendix}

\subsection{Solutions}

Table 5. Saline solution from (Wilson et al., 2004) as modified by (Zhang et al., 2013).

\begin{tabular}{|l|l|l|}
\hline Compound & Weight per litre of solution (g) & Final concentration (mM) \\
\hline $\mathrm{NaCl}$ & 6.019 & 103 \\
\hline $\mathrm{KCl}$ & 0.224 & 3 \\
\hline $\mathrm{MES}$ & 0.973 & 5 \\
\hline $\mathrm{NaHCO}_{3}$ & 2.184 & 26 \\
\hline $\mathrm{NaH}_{2} \mathrm{PO}_{3}$ & 0.138 & 1 \\
\hline $\mathrm{MgCl}_{2}\left({ }^{*} \mathrm{H}_{2} \mathrm{O}\right)$ & 0.813 & 4 \\
\hline Tetrahalose & 3.783 & 10 \\
\hline Saccharose & 2.401 & 7 \\
\hline
\end{tabular}

Saline solution preparation:

Mix all the compounds in $1000 \mathrm{~mL}$ of distilled water and adjust the $\mathrm{pH}$ to 7.25 with $\mathrm{NaOH} / \mathrm{HCl}$. For calcium imaging experiments add $0.294 \mathrm{~g}$ of $\mathrm{CaCl}_{2}\left({ }^{*} 2 \mathrm{H}_{2} \mathrm{O}\right)$ to obtain a final concentration of $2 \mathrm{mM}$ before starting the experiments.

Table 6. Epon recipe for electron transmission microscopy preparation.

\begin{tabular}{|l|l|}
\hline Compound & Weight per 100g (g) \\
\hline $\begin{array}{l}\text { Glycidether } 100 \text { (Serva, 21045, SERVA } \\
\text { Electrophoresis GmbH, Heidelberg, Germany) }\end{array}$ & 45.58 \\
\hline $\begin{array}{l}\text { DDSA (Serva, 20755, SERVA Electrophoresis GmbH, } \\
\text { Heidelberg, Germany) }\end{array}$ & 30.62 \\
\hline $\begin{array}{l}\text { MNA (Serva, 29452, SERVA Electrophoresis GmbH, } \\
\text { Heidelberg, Germany) }\end{array}$ & 23.75 \\
\hline
\end{tabular}

Epon preparation:

Mix all the compounds and stir for 10 minutes. Then add 1.8mL of DMP-30 (Serva, 36975, SERVA Electrophoresis $\mathrm{GmbH}$, Heidelberg, Germany) s 
Appendix

Table 7. Blocking solution

\begin{tabular}{|l|l|}
\hline Compound & Volume in PBS (\%) \\
\hline Normal goat serum (NGS) & $5 \%$ \\
\hline Bovine serum albumin (BSA) & $2 \%$ \\
\hline Triton & $1 \%$ \\
\hline
\end{tabular}

Blocking solution preparation:

Dissolve all compounds in PBS 7.4pH.

Table 8. Fly food recipe.

\begin{tabular}{|l|l|}
\hline Compound & Weight or volume per 7L \\
\hline Yeast & $500 \mathrm{~g}$ \\
\hline Sugar & $500 \mathrm{~g}$ \\
\hline Salt & $20 \mathrm{~g}$ \\
\hline Agarose & $60 \mathrm{~g}$ \\
\hline Four & $250 \mathrm{~g}$ \\
\hline Apple juice & $1 \mathrm{~L}$ \\
\hline Propionic acid & $30 \mathrm{~mL}$ \\
\hline Water & Add until volume reaches 7L \\
\hline
\end{tabular}




\subsection{Supplemental figures}
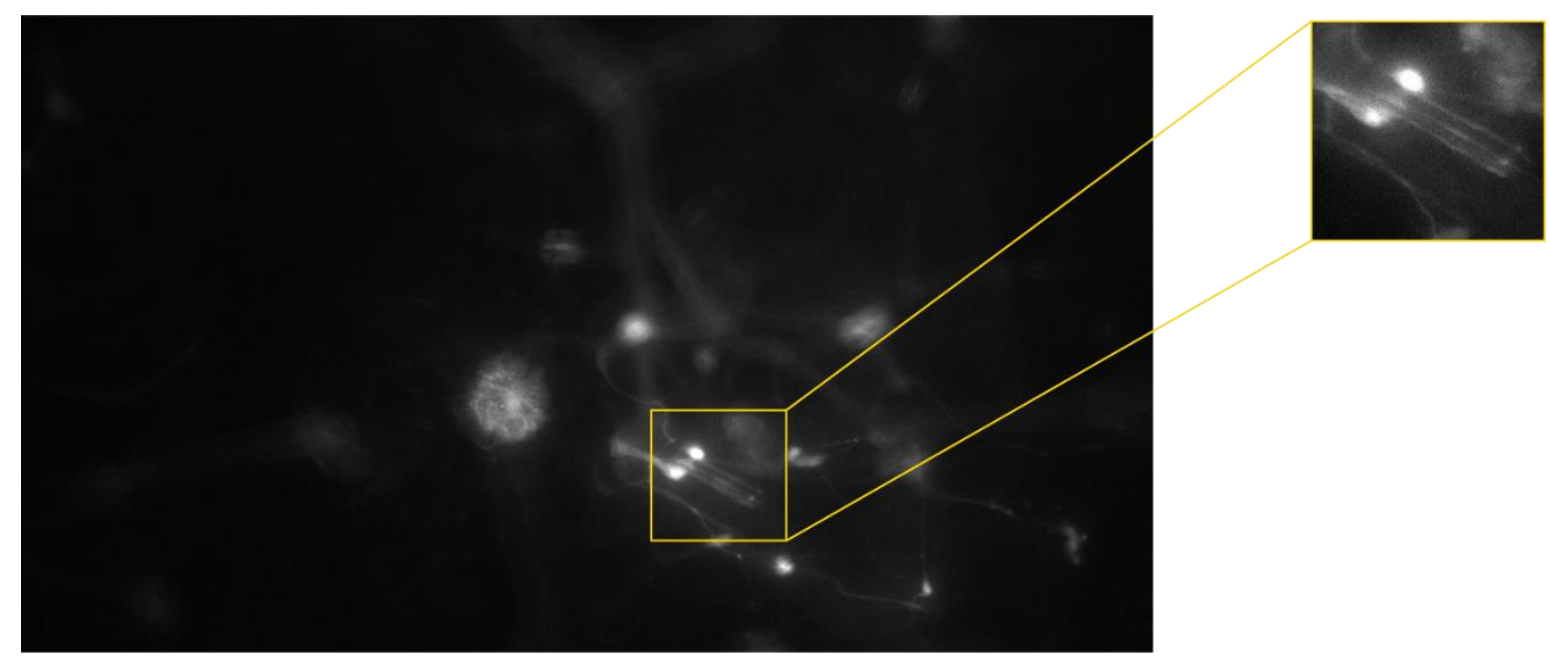

Figure 40. ROI to select chordotonal organ for calcium imaging. The image on the left shows the body wall neurons in the field of view (20X magnification), and the region of interest selected to image Ich5 neurons.
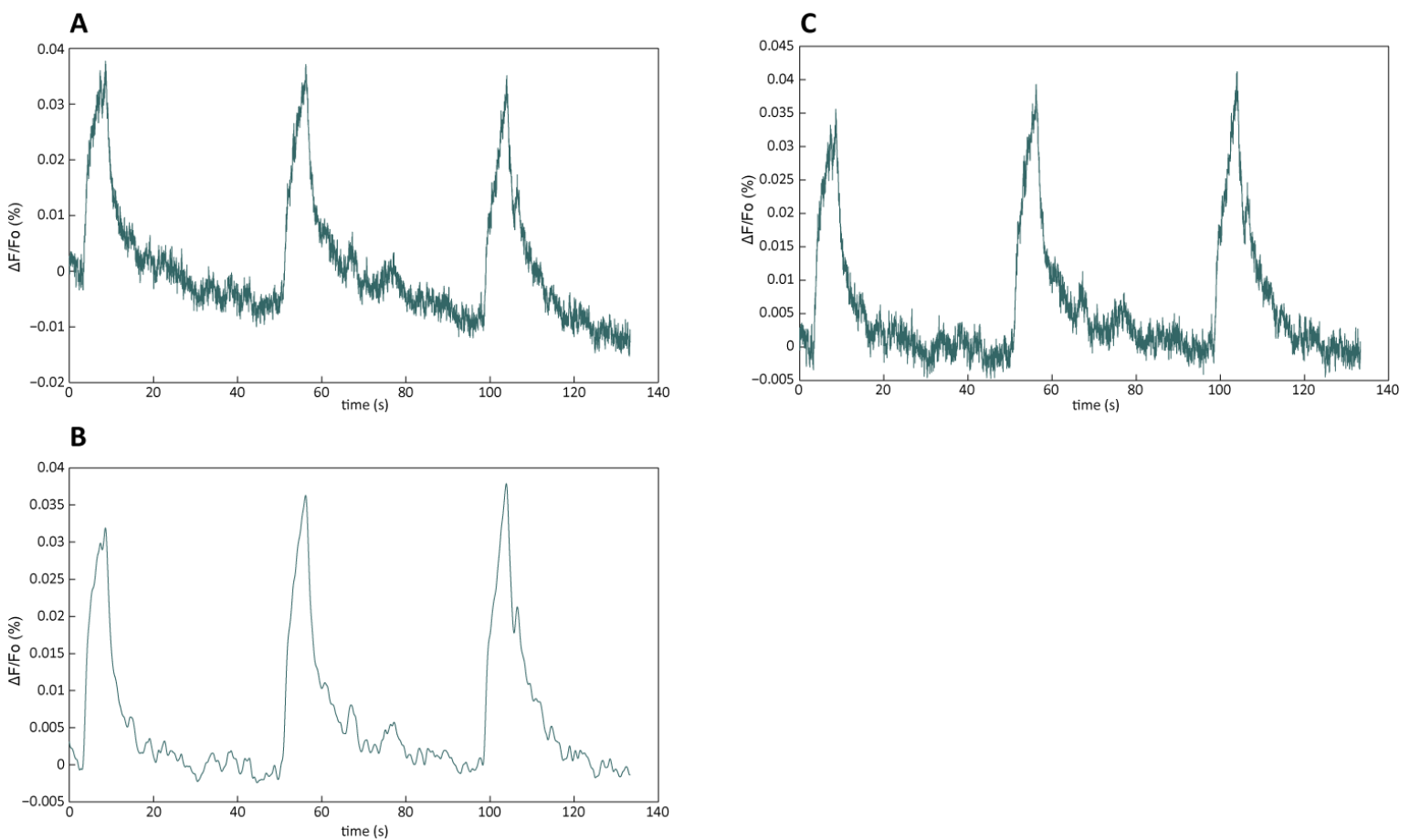

Figure 41. Bleach correction and smoothing of calcium signal. A) Original calcium trace. Photobleaching is apparent as the baseline signal between peaks seems to decay and is round -0.01 at the end of the recording. Digital noise is also apparent in the high frequency oscillations seen in the signal. B) Filtered calcium trace. C) Bleach corrected calcium trace. The bleach correction is carried out in FIJI using an exponential fit. The bleach corrected signal is smoothed using a Butterworth filter to reduce the digital noise seen in A and B. 


\section{Appendix}

A

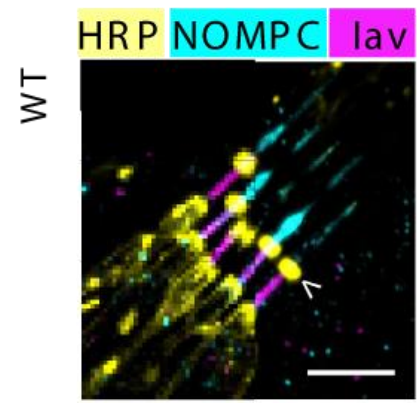

C

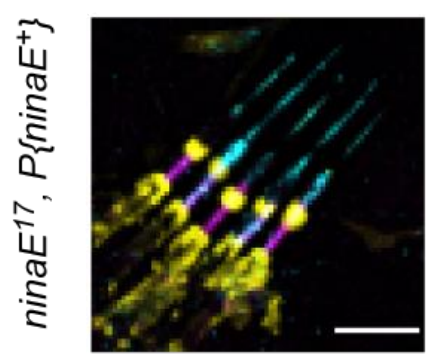

$\mathbf{F}$

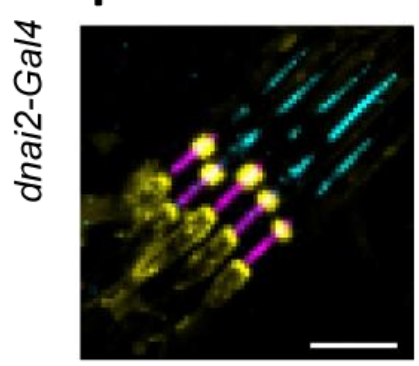

I

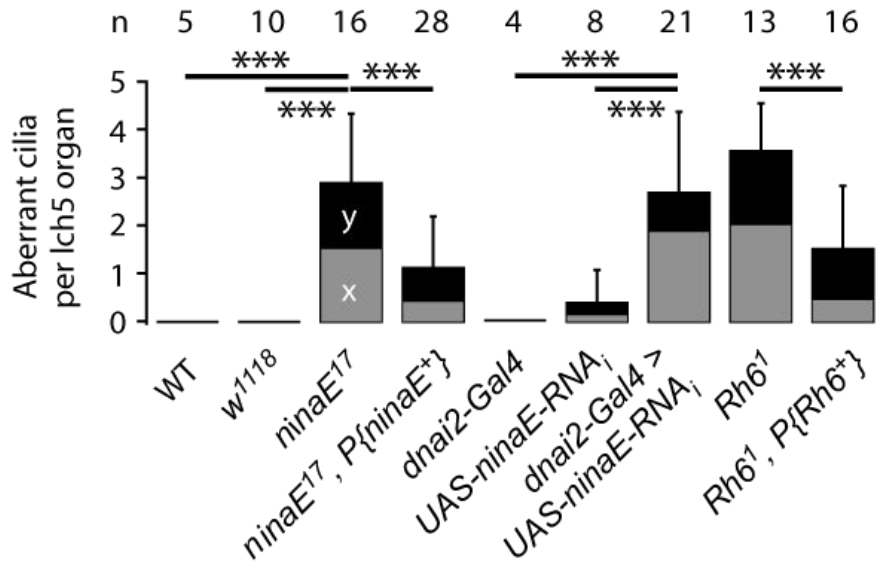

B

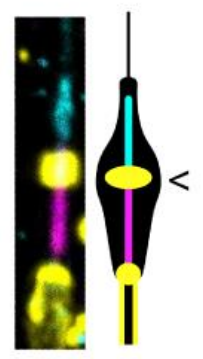

D

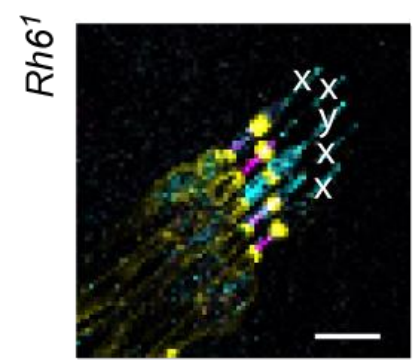

G
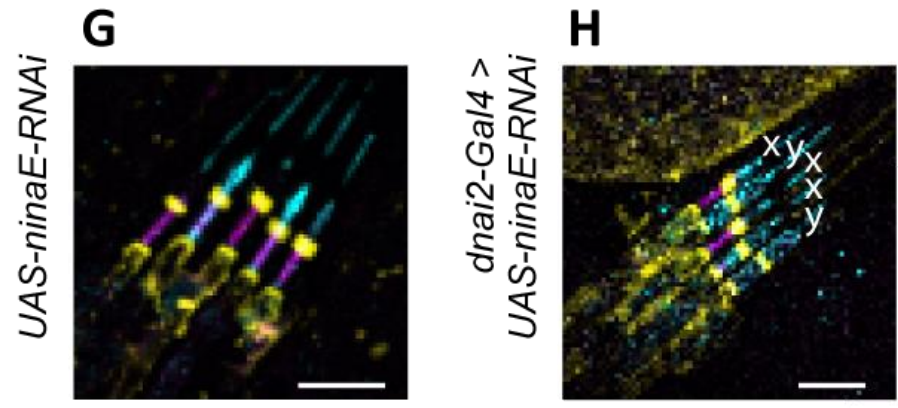

Figure 42. Opsin dependent mechanosensory channel localisation in Ich5 dendrites. A-H) NOMPC and Inactive localisation in Ich5 cilia revealed by anti-NOMPC (cyan) and anti-Inactive (magenta). Lch5 neurons are stained with antiHRP (yellow). The arrowhead indicates the 2nd HRP band dividing the cilia of the dendrite. A) Wild-type localisation of NOMPC is restricted to the distal ciliary region and Inactive localisation to the more proximal region (the panel on the right is a zoom-in and a sketch of the localisation in a single cilium). B) Channel localisation in nina $E^{17}$ mutants. In some dendrites NOMPC leaks into the endolymph space (s) and Inactive localisation is either unaffected (phenotype ' $x$ ') or missing (phenotype ' $y$ '). C) Channel localisation is rescued in nina $E^{17}, P\left\{n i n a E^{+}\right\}$. D) Channel mislocalisation is also found in $R h 6^{1}$ mutants. E) The $R h 6^{1}$ phenotype is rescued in $R h 6^{1}, P\left\{n i n a E^{+}\right\}$. F-G) dnai2-Gal4 and UAS-ninaE-RNAi show normal localisation. H) A knockdown of ninaE in chos leads to channel mislocalisation similar to the mutant. I) Numbers of aberrant cilia per Ich5 organ (means \pm S.D.). ' $x$ phenotype': grey, ' $y$ phenotype': black) ( $n=$ numbers of tested lch5 organs). Two-tailed Mann-Whitney U-tests with Bonferroni correction: ${ }^{*}=p<0.05,{ }^{* *}=p<0.01,{ }^{* * *}=p<0.001$. Scale bars (AH): $5 \mu \mathrm{m}$. Modified from Zanini, Giraldo et al. 2018. 


\section{List of abbreviations}

bd: bipolar dendrite

brv: $\quad$ brivido

CaMPARI: calcium-modulated photoactivatable ratiometric integrator

cho: chordotonal organ

CS18: $\quad$ Cantons flies reared at $18^{\circ} \mathrm{C}$

CS25: $\quad$ Cantons flies reared at $25^{\circ} \mathrm{C}$

C-opsin: ciliary opsin

da: dendritic arborisation

Dhc93AB: dynein heavy chain at 93AB

dnai2: $\quad$ axonemal dynein intermediate chain 2

es: $\quad$ external sensory organ

fco: femoral chordotonal organ

FTIR: $\quad$ frustrated total internal reflection

fps: $\quad$ frames per second

GCaMP6f: green fluorescent-calmodulin-M13 fusion protein 6 fast

GFP: $\quad$ green fluorescent protein

GPCR: G-protein coupled receptor

GR: gustatory receptor

HC: hot cell

hid: head involution defective

HRP: horseradish peroxidase

Hsp: heat shock protein

iav: inactive

IGLOO: Igloo is a Gradient Locomotion Model

IR: $\quad$ ionotropic receptor

Ich5: pentameric chordotonal organ

Mhc: $\quad$ myosin heavy chain

MD: $\quad$ multidendritic

nan: $\quad$ nanchung

ninaE: $\quad$ neither inactivation nor afterpotential $E$

norpA: $\quad$ no receptor potential A 
List of abbreviations

nSyb: neuronal-synaptobrevin

PBS: $\quad$ Phosphate-buffered saline

PBS-T: $\quad$ Phosphate-buffered saline Triton

PEs: $\quad$ Peltier elements

$\mathrm{PI} \quad$ preference index

PLC: $\quad$ phospholipase $\mathrm{C}$

RGR/Go: retinal Gprotein-coupled receptor, go-coupled plus retinochrome

Rh: Rhodopsin

rpr: reaper

RNAi: interference RNA

ROI: region of interest

R-opsin: rhabdomeric opsin

RWMC: $\quad$ Random Walk Monte Carlo

santa-maria: scavenger receptor acting in neural tissue and majority of rhodopsin is absent

$\mathrm{td}$ : tracheal dendrite

TRP: $\quad$ transient receptor potential

TRPA: $\quad$ transient receptor potential ankyrin

TRPC: $\quad$ transient receptor potential canonical

TRPM: $\quad$ transient receptor potential melastatin

TRPML: $\quad$ transient receptor potential mucolipin

TRPN: $\quad$ transient receptor potential nompC

TRPP: $\quad$ transient receptor potential polycystin

TRPV: $\quad$ transient receptor potential vanilloid

WT: $\quad$ wild-type 


\section{List of figures}

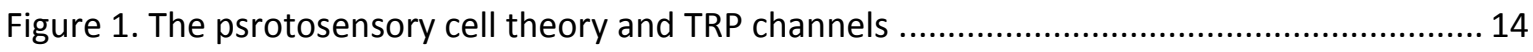

Figure 2. Sensory body wall neurons of a larval hemisegment .................................................... 17

Figure 3. Larval pentameric chordotonal organs (Ich5) .................................................................. 19

Figure 4. Larva and adult locomotion setups and tracing .......................................................... 24

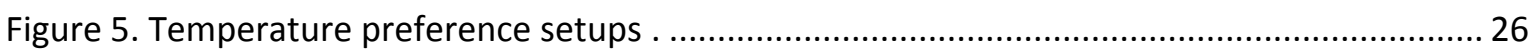

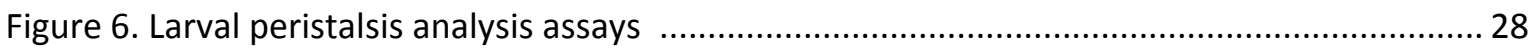

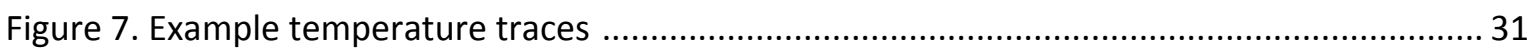

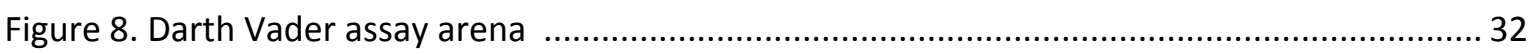

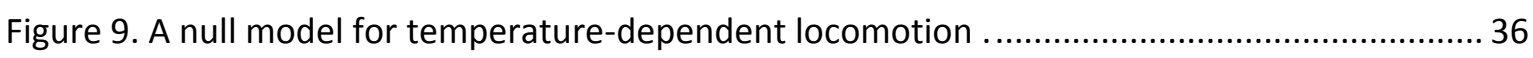

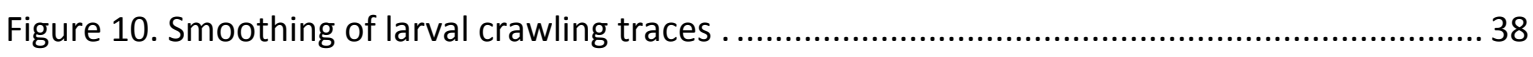

Figure 11. Fillet preparation and calcium imaging of Ich5 organ neurons ....................................... 41

Figure 12. Thermosensory molecules of Drosophila melanogaster . .............................................. 50

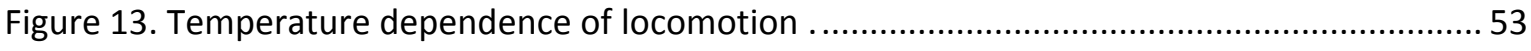

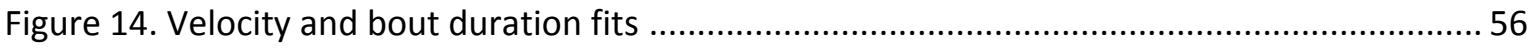

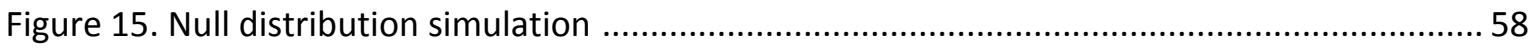

Figure 16. Proportion of cold trapped animals after the simulation ............................................. 59

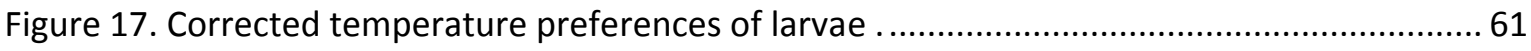

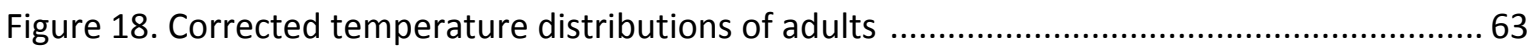

Figure 19. Corrected temperature preferences and start of cold and hot avoidance of adults ..... 65

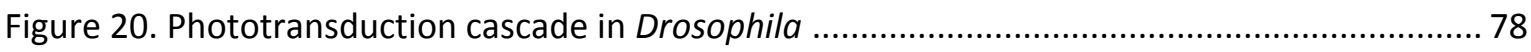

Figure 21. Mechanosensory channels of chordotonal organs ................................................... 81

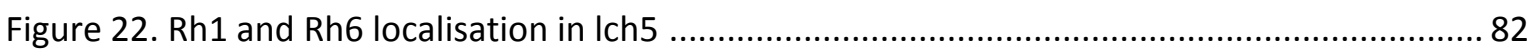

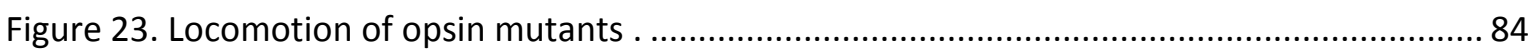

Figure 24. Locomotion of mechanosensory ion channel mutants and ninaE $E^{17}-R h 6^{1}$ double mutants

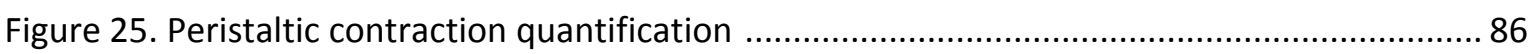

Figure 26. Locomotion of chordotonal receptor-ablated larvae and ninaE chordotonal knockdown

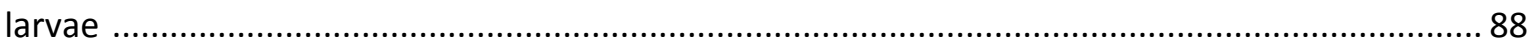

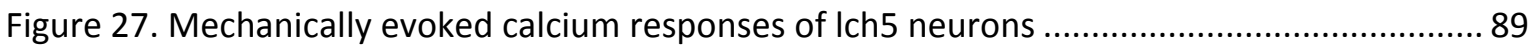

Figure 28. Locomotion of $d$ trpA1 receptor-ablated larvae and mutant larvae at $29^{\circ} \mathrm{C}$................... 91

Figure 29. Temperature preferences of larvae with a cho specific ninaE knockdown ................... 92

Figure 30. Locomotion of santa-maria mutants and vitamin A depleted larvae.............................. 94 
Figure 31. Locomotion of norpA mutants and norpA expression in Ich5 neurons ......................... 96

Figure 32. Transmission electron images of Ich5 neuron dendrites ............................................ 98

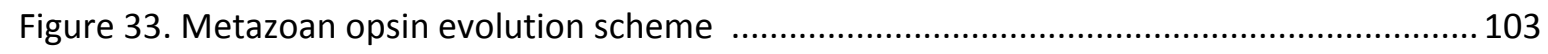

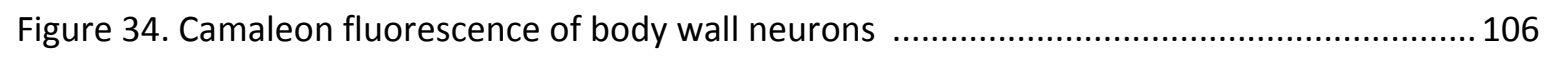

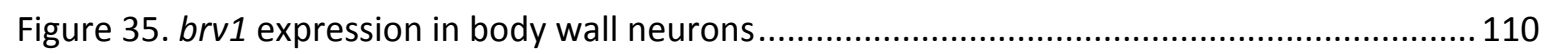

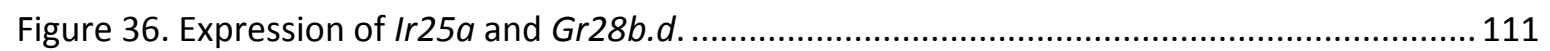

Figure 37. Temperature preferences of larvae with a cho specific brv1 knockdown................... 113

Figure 38. CaMPARI imaging of Ich5 neurons stimulated with temperature ................................ 115

Figure 39. Locomotor performance of cho specific brv1 knockdown and brv2 mutants, and

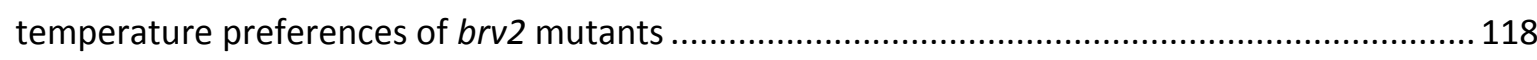

Figure 40. ROI to select chordotonal organ for calcium imaging ................................................ 139

Figure 41. Bleach correction and smoothing of calcium signal................................................... 139

Figure 42. Opsin dependent mechanosensory channel localisation in Ich5 dendrites.................. 140 


\section{List of tables}

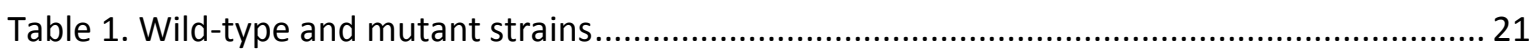

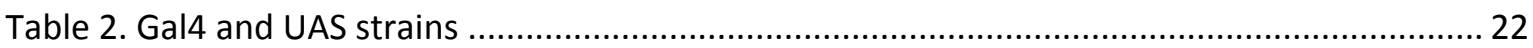

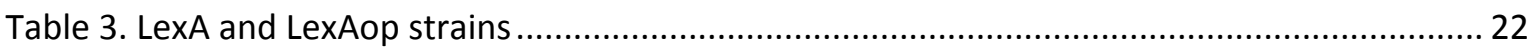

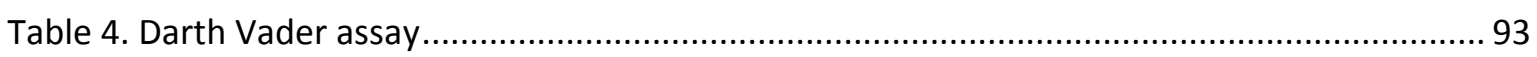

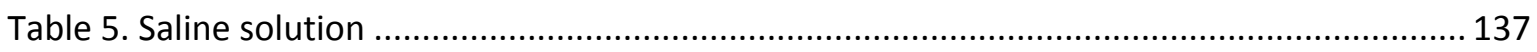

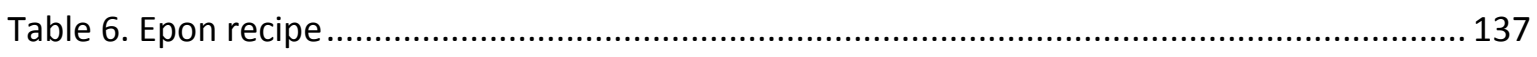

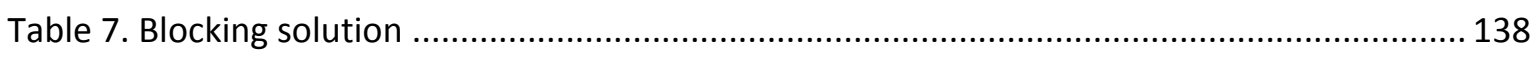

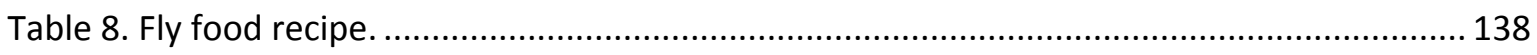




\section{Acknowledgements}

First of all, I would like to thank Dr. Bart Geurten for all the time and effort he put into this project. I am thankful for not only being a great mentor and inspiration, but a great friend. Thank you for all the teachings, support, and helping me become a better scientist.

I would like to thank Prof. Dr. Martin Göpfert for the guidance and support throughout the project, and the opportunity to work in his lab.

I thank the members of my thesis committee Prof. Dr. André Fiala and Dr. Manuela Schmidt for their input and fruitful discussions that helped me finish my work.

I also want to thank Dr. Damiano Zanini for working with me on this project and teaching me everything I know about fly genetics. I would have never gotten so far without all your help and constant discussions of the project. Additionally, I thank Dr. Marta Andrés for all her help in the beginning of the project. I hope we get to work again together one day and get rid of all the mosquitoes in the world. I thank Radoslaw Katana for all his help in the final phase of the project, teaching me how to do stainings and always being available to discuss and talk, whether it was about science or football. Dr. Christian Spalthoff and Dr. Philipp Jähde for their help setting up the calcium imaging. Andrea Adden for her input at the beginning of the project and providing the locomotion data for adults in chapter 1.

I thank Kristina Corthals and Robert Kossen for being my lab family these last years. Thank you for making the lab a great place to go to everyday and all the great times we shared. Krissy, thank you for the help with the revision of the thesis and for the company in all the cool trips to distant exotic lands, I hope we get to keep the tradition throughout the years. Robert thank you for all the discussions and your constant input and help with the project. I also thank llyas for making the lab so much fun the little time he was there, and all the great people that came through our office in these years. I also thank Luis Ramos for the coffee breaks and long discussions and ideas for the project.

Stephanie Pauls thank you for all the help with the stainings and fly care, and Nicola SchwedhelmDomeyer for the EM images. Jan Hoffman, Tobias Mühmer, and Sven Neumann for the great work building all the set ups used in this work, and the constant technical support. I thank all the members of the Department of Cellular Neurobiology for their input to the project. 
Sharlen Moore, thank you for all the patience, constant support, your help through tough times, and you company through the good times. I could have never done this without you. Thank you especially for being my family in Germany.

To all my friends in Germany that made these last four years amazing, thank you for all your support and the great memories.

Finalmente, gracias a mi familia por ayudarme a llegar hasta acá. A mi mamá Sandra y a mi papá Francisco, gracias por todo el apoyo y el amor, y por llevarme a ser lo que hoy soy. A Cami, gracias por el apoyo desde la distancia y siempre estar pendiente y orgullosa de mis logros. A mis abuelos, Hernando e Inés, este trabajo se lo dedico a ustedes, porque sin su amor y dedicación no hubiera llegado hasta acá. 


\section{Declaration}

Herewith I declare, that I prepared the doctoral thesis "Linking senses: the genetics of Drosophila larval chordotonal organs" on my own and with no other sources and aids than quoted.

Göttingen, $29^{\text {th }}$ of March, 2018.

Diego Alejandro Giraldo Sánchez 


\section{Curriculum Vitae}

Personal Information

Name: Diego Alejandro Giraldo Sánchez

Date of birth: $\quad$ February $26^{\text {th }}, 1989$

Place of birth: Bogota, Colombia

Nationality: Colombian

Education

April 2014-

Present

October 2012 -

March 2014

2007-2012:

\section{Georg-August Universität Göttingen}

PhD Neuroscience

Thesis title: Linking senses: the genetics of Drosophila larval chordotonal organs

Programme: International Max Planck Research School for Neurosciences Department for Cellular Neurobiology

Supervisors: Prof. Dr. Martin Göpfert and Bart Geurten PhD

Committee members: Prof. Dr. André Fiala, Dr. Manuela Schmidt

Georg-August Universität Göttingen

Göttingen, Germany

\section{Georg-August Universität Göttingen}

MSc Neuroscience

Program: International Max Planck Research School for Neurosciences

Department for Cellular Neurobiology

Supervisors: Prof. Dr. Martin Göpfert and Bart Geurten PhD

Georg-August Universität Göttingen

Göttingen, Germany

Master studies examination: A

\section{Universidad de los Andes}

BSc Biology

Department of Biological Sciences

Bogota, Colombia

\section{Publications}

Zanini, D.*, Giraldo, D.*, Warren, B. Katana, R., Andrés, M., Reddy, S., Pauls, S., SchwedhelmDomeyer, N., Geurten, B.R.H., and Göpfert, M.C., Proprioceptive Opsin Functions in Drosophila Larval Locomotion. Neuron (2018).

Giraldo, D., Hernández, C., Molina, J., 2013. In search of magnetosensitivity and ferromagnetic particles in Rhodnius prolixus: Behavioral studies and vibrating sample magnetometry. Journal of Insect Physiology 59, 345-350

*These authors contributed equally 
Scholarships

2014 April-

Present

2012 October -

2013 September
DAAD Scholarship for Doctoral student for more than 6 months Scholarship from the German Academic Exchange (DAAD) for PhD students.

Stipend of the excellence foundation for the promotion of the Max Planck Society

Stipend awarded for Master studies in the IMPRS for Neurosciences

Research Experience

2014 April -

Present

2013 October 2014 March

2013 May July

2013 March May

2013 January March

2012 January August

\section{Doctoral Thesis}

Linking senses: the genetics of Drosophila larval chordotonal organs Department for Cellular Neurobiology Georg-August Universität Göttingen Göttingen, Germany

\section{Master Thesis}

The role of Rhodopsin in thermoreception and proprioception: A neuroethological approach.

Department for Cellular Neurobiology

Georg-August Universität Göttingen

Göttingen, Germany

\section{Lab Rotation}

Effects of Large Field Neurons of the Ellipsoid Body Lateralization in the Walking Direction Bias of Drosophila melanogaster Department for Molecular Neurobiology of Behaviour Georg-August Universität Göttingen

Göttingen, Germany

\section{Lab Rotation}

Manipulating stimuli presentation order in Induced Roelofs Effect illusion DPZ (German Primate Center)

Sensorimotor Group

Georg-August Universität Göttingen

Göttingen, Germany

\section{Lab Rotation}

Is Rhodopsin 6 Involved in Thermoreception in Drosophila melanogaster? Department for Cellular Neurobiology Georg-August Universität Göttingen

Göttingen, Germany

\section{Research Assistant}

Behavioral effects of low power radio frequencies on Chagas Disease vector Rhodnius prolixus

CIMPAT (Center of Investigations in Microbiology and Tropical

Parasitology) and Magnetism Laboratory

Universidad de los Andes 
Bogota, Colombia

2011

Bachelor Thesis Research

Magnetoreception in Rhodnius prolixus: magnetic properties and

behavioral experiments

CIMPAT (Center of Investigations in Microbiology and Tropical

Parasitology) and Magnetism Laboratory

Universidad de los Andes

Bogota, Colombia

Teaching Experience

2016 December

2016 March -

April

2016 January -

February

2014 December

2014 December

2011 August -

December

2011 August -

December

2011 August -

December
Teacher Assistant in Practical Course Sensory and Behavioral Physiology The neuroethology of weakly electric fish

Georg-August Universität Göttingen

Göttingen, Germany

Lab Rotation Supervisor

IMPRS Neuroscience Program

Georg-August Universität Göttingen

Göttingen, Germany

Lab Rotation Supervisor

IMPRS Neuroscience Program

Georg-August Universität Göttingen

Göttingen, Germany

Teacher Assistant in Practical Course Sensory and Behavioral Physiology The neuroethology of weakly electric fish

Georg-August Universität Göttingen

Göttingen, Germany

Teacher for the Practical Course Neurobiology I

Motion processing vision in Drosophila

Developmental, Neural and Behavioral Biology Program

Georg-August Universität Göttingen

Göttingen, Germany

Teacher Assistant in Biology of Organisms Laboratory Class

Universidad de los Andes

Bogota, Colombia

Teacher Assistant in Biostatistics Laboratory Class

Universidad de los Andes

Bogota, Colombia

Teacher Assistant in Biology for Psychology Laboratory Class

Universidad de los Andes

Bogota, Colombia 
Curriculum Vitae

2011 January - May Teacher Assistant in Biology for Psychology Laboratory Class

Universidad de los Andes

Bogota, Colombia

2010 August - $\quad$ Teacher Assistant in Biology for Psychology Laboratory Class

December

Universidad de los Andes

Bogota, Colombia

Professional Development

2018

PH425x: MalariaX: Defeating Malaria from the Genes to the Globe edx, Inc. (MOOC platform)

Online course offered by Harvard University

Languages

Spanish (native language)

English (Fluent)

French (Fluent)

Italian (Intermediate)

German (A2) 